Source attribution of human toxoplasmosis

A quantitative microbial risk assessment approach
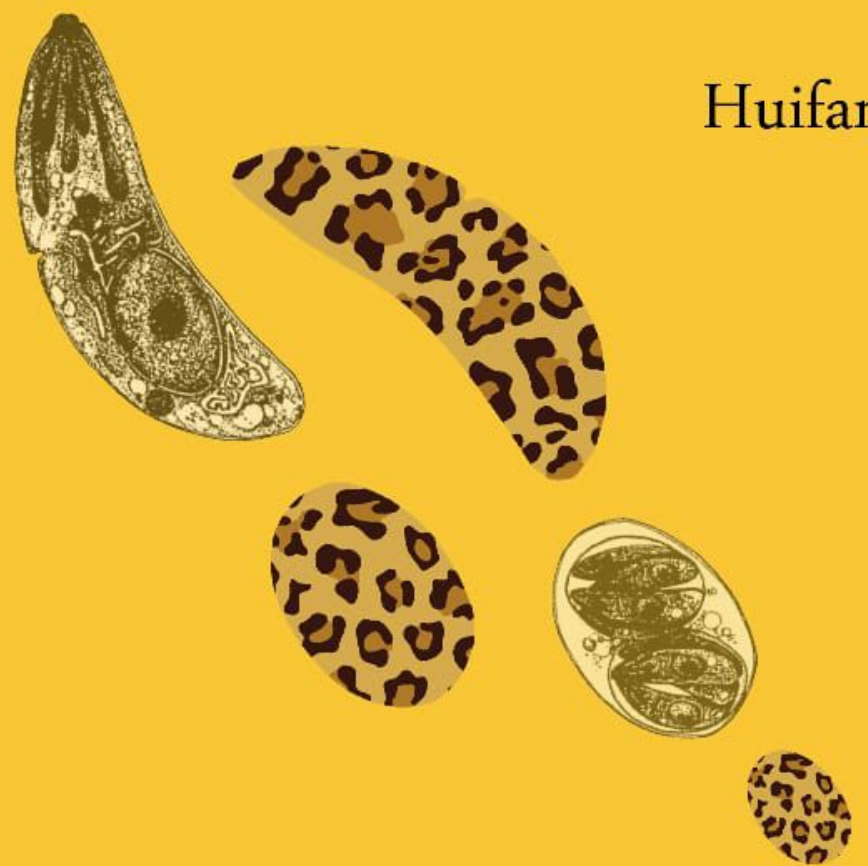

\%ิ:

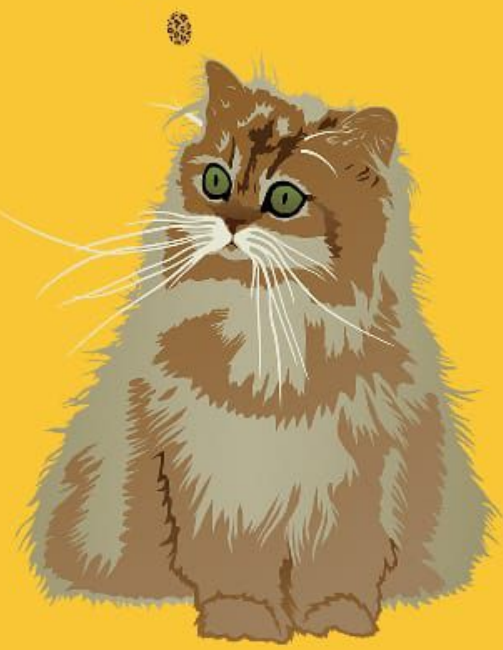




\title{
Source attribution of human toxoplasmosis
}

A quantitative microbiological risk assessment approach

\author{
Huifang Deng
}




\section{Source attribution of human toxoplasmosis}

A quantitative microbiological risk assessment approach

Huifang Deng, 2020

PhD dissertation, Utrecht University, the Netherlands

Cover design: Kim Ouweleen (www.murugandi.com)

Interior design: Huifang Deng (huifangdeng.github.io)

Printing: Ipskamp Printing

ISBN: 978-94-6421-127-6

The research presented was conducted at the National Institute for Public Health and the Environment (RIVM), Bilthoven, the Netherlands.

All rights reserved. No part of this publication may be reproduced or transmitted in any form or by any means, without permission in writing from the author. The copyright of the papers that have been published or have been accepted for publication has been transferred to the respective journals. 


\title{
Source attribution of human toxoplasmosis
}

A quantitative microbiological risk assessment approach

\author{
Bronattributie van humane toxoplasmose \\ Een kwantitatieve microbiologische risicoschatting \\ (met een samenvatting in het Nederlands)
}

\section{Proefschrift}

\author{
ter verkrijging van de graad van doctor aan de \\ Universiteit Utrecht \\ op gezag van de \\ rector magnificus, prof.dr. H.R.B.M. Kummeling, \\ ingevolge het besluit van het college voor promoties \\ in het openbaar te verdedigen op \\ dinsdag 8 december 2020 des ochtends te 11.00 uur
}

door

\section{Huifang Deng}

geboren op 6 mei 1988

te Hunan, China 


\section{Promotor:}

Prof. dr. D.J.J. Heederik

\section{Copromotoren:}

Dr. J.W.B. van der Giessen

Dr. M. Opsteegh

Dit proefschrift werd (mede) mogelijk gemaakt met financiële steun van het Ministerie van Volksgezondheid, Welzijn en Sport. 


\section{Assessment committee:}

Prof. dr. A. Havelaar

Prof. dr. A. Gröne

Prof. dr. J.A. Stegeman

Prof. dr. M.E.E. Kretzschmar

Prof. dr. M. Liu

Dr. L. Mughini-Gras

\section{Paranymphs}

Dr. A. Swart

P.D. Hengeveld 



\section{TABLE OF CONTENTS}

$\begin{array}{lll}\text { Chapter } 1 & \text { General introduction } & 9\end{array}$

PART I: $\quad$ MULTI-HOST TRANSMISSION

Chapter 2 Mathematical modelling of Toxoplasma gondii

transmission: A systematic review

PART II: $\quad$ EPIDEMIOLOGICAL INSIGHTS

Chapter 3 Risk factors related to Toxoplasma gondii

seroprevalence in indoor-housed Dutch dairy goats

Chapter 4 Seroprevalence of Toxoplasma gondii in pregnant women and livestock in the mainland of China: a systematic review and hierarchical meta-analysis

PART III: $\quad$ QUANTITATIVE MICROBIAL RISK ASSESSMENTS

Chapter 5 The effect of salting on $T$. gondii viability evaluated and implemented in quantitative risk assessment of meat-borne Toxoplasma gondii infection

Chapter 6 Quantitative risk assessment of meat-borne Toxoplasma gondii infection in the mainland of China

Chapter 7 Digging into Toxoplasma gondii infections via soil: a quantitative microbial risk assessment approach

Chapter 8 General discussion

ADDENDUM Summary (in English and Dutch)

List of publications

Acknowledgements

About the author 



\section{Chapter}

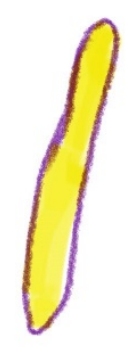

General introduction 
10 . CHAPTER 1 


\section{THE PARASITE}

Toxoplasma gondii (T. gondii) is a single-celled zoonotic parasite, which has a worldwide distribution. It can infect virtually all warm-blooded animals, including humans, mammals and birds. It was first discovered in Tunisia and Brazil in 1908 and named based on its crescent-shaped morphology (Toxoplasma means 'arc form' in Greek) and the host species (a rodent, Ctenodactylus gundi) in which they were detected a year after (Dubey, 2008). It belongs to the phylum of Apicomplexa together with other species, but is the only known member of the genus Toxoplasma.

\section{PARASITIC STAGES}

There are three infective stages of $T$. gondii: tachyzoites in the bloodstream, bradyzoites in tissue cysts, and sporozoites in oocysts (Dubey, 1998). These infective stages are approximately $5 \mu \mathrm{m}$ long, $2 \mu \mathrm{m}$ wide, and bow-shaped cells (Robert-Gangneux and Darde, 2012). Tachyzoites are able to invade all of the nucleated cells of warm-blooded animals, where they replicate every 6 to 8 hours (Halonen and Weiss, 2013). Bradyzoites result from the transformation of tachyzoites, and are a slow dividing stage of $T$. gondii. Bradyzoites are surrounded by cyst walls and located in multiple organs and muscles, including the central nervous system tissue and the heart. Tissue cysts vary in size from $10 \mu \mathrm{m}$ (containing only two bradyzoites) to $100 \mu \mathrm{m}$ (containing hundreds or thousands of bradyzoites). The oocyst containing sporozoites, is an environmental stage of the parasite. Sporulated oocysts have an approximate size of $12 \mu \mathrm{m}$, and contain two sporocysts, each sporocyst containing four sporozoites. Sporulated oocysts are environmentally resistant and can survive for more than a year in a moist environment above freezing temperature (Frenkel et al., 1975; Lelu et al., 2012). Oocysts have also been found to survive in seawater for up to 6 months (Lindsay and Dubey, 2009) enabling transmission in coastal marine environments. Moreover, oocysts have been shown to adhere to berries (Kniel et al., 2002), suggesting raw vegetables/fruits could be sources of infection.

\section{LIFE CYCLE}

T. gondii has a complex life cycle which involves sexual and asexual reproduction, definitive hosts and intermediate hosts. Cats and other felids are the only known definitive hosts of $T$. gondii, where sexual reproduction takes place in the epithelial cells of the small intestine (Dubey, 1998). They can become infected after ingesting any of the three infectious stages of $T$. gondii (Miller et al., 1972) and are able to excrete millions of unsporulated oocysts in feces into the environment (Dubey et al., 1970). The time to excretion of oocysts after infection (prepatent period) varies according to the life cycle stage ingested, with the shortest prepatent period from tissue cysts (3-10 days), then tachyzoites ( 13 days), and the longest period from oocysts (18 days) (Dubey, 1998). The excretion of T. gondii oocysts lasts for 1 to 2 
weeks after primary infection of the definitive hosts (Dubey, 1995). Depending on humidity and temperature, infectious oocysts (sporulation) develop 1 to 3 days after deposition (Dubey et al., 1970). They can be dispersed into the environment by wind, run-off from soil to water and vectors. Sporulated oocysts can infect a wide range of intermediate hosts. Recently, it has been reported that the deficiency in delta-6-desaturase activity and the buildup of linoleic acid in the small intestines of felines make them the only suitable hosts for $T$. gondii sexual replication. An experimental study in mice showed that after inhibition of delta-6desaturase, oocysts were produced in mice feces, and these mouse-derived sporulated oocysts were stable and infectious for at least 3 months when stored at $4{ }^{\circ} \mathrm{C}$ (Martorelli Di Genova et al., 2019).

All warm-blooded animals serve as intermediate hosts of $T$. gondii, where only asexual development occurs. After consuming water, vegetables or fruits contaminated with sporulated oocysts, sporozoites are released in the gut lumen, penetrate the intestinal epithelium and differentiate into tachyzoites (Hill and Dubey, 2002). Tachyzoites rapidly replicate and disseminate through the body by blood circulation. Conversion from tachyzoites to bradyzoites occurs afterwards and matures into tissue cysts that have a thick wall. Tissue cysts develop intracellularly and remain in the organs and muscles in most hosts in a dormant stage (Montoya and Liesenfeld, 2004). It is generally believed that cysts may remain viable for the life of the host, triggering life-long immunity, but this concept has recently been challenged (Rougier et al., 2017). If a carnivorous host ingests tissue cysts through raw or undercooked meat, bradyzoites will be released from ruptured cysts and infect the intestinal epithelium of the new host. These bradyzoites will transform back to rapidly dividing tachyzoites and disseminate through the body.

In addition to horizontal transmission, the parasite can also be vertically transmitted in all warm blooded animals including humans. If a primary infection occurs during or just before pregnancy, tachyzoites can cross the placenta and infect the fetus (congenital toxoplasmosis, CT).

\section{TRANSMISSION ROUTES OF HUMAN T. GONDII INFECTION}

The main transmission routes for postnatal human $T$. gondii infection are through consumption of raw/undercooked meat (e.g., farm animals or game meat) containing viable tissue cysts, and through ingestion of oocysts from contaminated vegetables, water and soil (Tenter et al., 2000). So far, the relative importance of transmission via tissue cysts versus oocysts in a given population is largely unknown, and may vary depending on culture and consumption habits. Infections through consuming unpasteurized milk, from tachyzoites in tissue transplants and blood products have also been reported. 


\section{TOXOPLASMOSIS}

$T$. gondii infection has a worldwide distribution, and it is generally assumed that one third of the world's human population is infected. The prevalence of infection varies widely between countries and often within a given country (Pappas et al., 2009). In immunocompetent individuals, most infections are asymptomatic, because the immune system usually keeps the parasites from causing illness. Occasionally, some people may experience flu-like symptoms (e.g., fever, muscle aches, etc.) and swollen lymph nodes for a few weeks or months (Halonen and Weiss, 2013). It was recognised that postnatally acquired $T$. gondii infection in immune-competent people can cause chorioretinitis (ocular toxoplasmosis) (Gilbert and Stanford, 2000). However, severe consequences are more common in two main risk groups: pregnant women and immuno-compromised individuals (Montoya and Liesenfeld, 2004).

Generally, if women acquire $T$. gondii infection before pregnancy, the unborn child will be protected, because the mother has developed immunity. However, if primary infection occurs during or just before pregnancy, congenital infection may take place in about $30 \%$ of cases (Dunn et al., 1999). The probability of vertical transmission increases with gestational age, but the consequences for the offspring are more severe in early stages of pregnancy (e.g., major abnormalities in the brain and eye tissues or abortion) (Thiebaut et al., 2007). Infected children who are healthy at birth may develop chorioretinitis later in life.

Since parasites remain in the infected person's body in an inactive stage, they can become reactivated if the person becomes severely immunosuppressed, e.g., patients receiving corticosteroids or cytotoxic drugs, or patients with organ transplants or infected with HIV. The disease can be life threatening in immunocompromised patients.

\section{BURDEN OF DISEASE}

The mortality and morbidity burden of toxoplasmosis can be quantified by using an integrated metric called disability-adjusted life years (DALYs). It is the sum of years of life lost and years lived with disability, weighted for the severity of the illness across the whole population (Havelaar et al., 2012). One DALY is equivalent to one year of healthy life lost. Congenital infection is the most important part in estimating the disease burden of toxoplasmosis. It was estimated that the annual global incidence of CT was 1.5 cases per 1,000 live births, and the burden of CT was estimated to be 1.2 million DALYs (95\% Cl: 0.76-1.90) in 2012. High disease burdens were found in the South America region and the Eastern Mediterranean region. Among sub-regions in Europe, the estimated incidence rates of CT varied from 0.5 to 1.6 cases per 1,000 live births (Torgerson and Mastroiacovo, 2013). In the Netherlands, the observed incidence rate of CT was 2 per 1,000 live births in 
2006 (Kortbeek et al., 2009), and the estimated disease burden of CT in 2018 was 1,900 DALYs (Pijnacker et al., 2019). This incidence rate of CT was ten times higher than what was found in Denmark based on estimates obtained using the same methods (Nissen et al., 2017; Schmidt et al., 2006).

When using DALYs to compare the global disease burden among 31 major foodborne pathogens, $T$. gondii ranked $13^{\text {th }}$, and contributed significantly to the food-borne disease burden in Central and South America subregions (Havelaar et al., 2015). It also ranked highly among 11 foodborne parasitic diseases, and caused 825,000 DALYs (95\% UI: 561,000-1.26 million) globally in 2010 (Torgerson et al., 2015). At the national level, it was estimated that $T$. gondii was associated with one of the highest disease burdens among fourteen important food-related pathogens in the Netherlands (Havelaar et al., 2012; Mangen et al., 2015; Mangen et al., 2017; Pijnacker et al., 2019).

In addition, the risk of infection can be quantified in terms of economic impact in a given population. This can be done by calculating the cost-of-illness (COI), and it takes healthcare costs, costs for the patient and/or his family, such as travel expenses, as well as costs in other sectors (e.g., due to productivity loss) into account. In 2018, the annual cost-of-illness for $T$. gondii was estimated to be $€ 15$ million in the Netherlands (Pijnacker et al., 2019). In a multicriteria-based ranking which took public health, trade, and socio-economic impact aspects into account, $T$. gondii ranked second out of 24 food-borne parasites at the European level (Bouwknegt et al., 2018).

\section{INTERVENTION STRATEGIES}

The high disease burden of toxoplasmosis means that possibilities for intervention should be considered. Several potential intervention measures targeting either congenital disease, meat-borne transmission or contamination of the environment have been suggested to reduce the disease burden of human infection (Opsteegh et al., 2015). Currently, in the Netherlands, intervention mainly focused on preventing congenital toxoplasmosis by educating pregnant women about the sources of infection (primary prevention). So far, except for health education, only a few countries (Austria, Belgium, France, Portugal, Slovakia and Slovenia) have active surveillance and treatment of congenital cases by mandatory screening of pregnant women (secondary prevention), and additional intervention measures targeting the sources of infection are generally not in place (ECDC, 2019). However, these screening programs are costly and the debate about the usefulness of secondary prevention is still ongoing due to the unclear beneficial effect of treatment. Other potential approaches are increasing biosecurity on meatproducing livestock farms, and vaccinating cats. Strict measures (e.g., rodent control, keeping cats away from farm/bedding material/storage, and providing 
clean drinking water) are generally implemented for indoor-reared animals, but are less feasible for animals with outdoor access. Using serological tests to identify T. gondii infected animals/high-risk farms may be useful, but only when there is a good correlation between seropositivity and the presence of tissue cysts in the animal species (e.g., pig, and small ruminant) (Opsteegh, et al., 2016a; Opsteegh, et al., 2016b). So far, there is no commercially available vaccine for use in cats and humans. Although there is a vaccine that has been shown to reduce tissue cyst formation in sheep (Katzer et al., 2014) and pigs (Burrells et al., 2015), it is licensed and used only to prevent abortions in sheep. In addition, based on mathematical models, high cat vaccination coverage is needed to reduce the number of human infections, and a high coverage was considered unfeasible for large cat populations (Bonačić Marinović et al., 2019; Turner et al., 2013).

Implementing measurements to inactivate $T$. gondii tissue cysts or oocysts in food might be practical for consumers to reduce disease burden of human toxoplasmosis. The parasite cannot grow outside of a host or in food and the environment, and the parasite can be inactivated in several ways. Studies showed that thermal (heating and freezing) and non-thermal methods (high pressure, irradiation and curing) are effective for making the parasite inviable (Mirza Alizadeh et al., 2018). Effective measurements to prevent $T$. gondii infection are adequate cooking and freezing. It has been recommended that meat should be cooked thoroughly to $>67^{\circ} \mathrm{C}$ before consumption and stored for at least two days at $-12{ }^{\circ} \mathrm{C}$ to inactivate $T$. gondii tissue cysts in contaminated meat (Voedingscentrum, 2020). Oocysts can lose the ability to sporulate when exposed to freezing $\left(-21^{\circ} \mathrm{C}\right.$ for 1 day, or $-6^{\circ} \mathrm{C}$ for 7 days) and can be inactivated after heating at $50^{\circ} \mathrm{C}$ for 10 minutes (Dubey et al., 1970; Frenkel and Dubey, 1973). Recently, a social cost-benefit analysis was performed to evaluate the net value of two potential interventions (i.e., freezing meat intended for raw or undercooked consumption and improving biosecurity in pig farms) for the Dutch society. The results showed that freezing steak tartare and leg of mutton would lead to net benefits for society (Suijkerbuijk et al., 2019). However, freezing of meat to inactivate $T$. gondii and to improve food safety was not favoured by the Dutch consumers (Lambooij et al., 2019).

\section{SOURCE ATTRIBUTION}

Reducing the burden of toxoplasmosis is challenging since current intervention mainly focuses on primary prevention. Primary prevention requires identification of the most important sources causing disease and prioritization of effective intervention strategies. The term "source attribution" is defined as the partitioning of the human disease burden of one or more foodborne infections to specific sources (e.g., food and animal reservoirs) (Pires et al., 2009). For this purpose, a variety of methods to estimate the relative contribution of different 
sources of $T$. gondii infections have been applied worldwide. Approaches to source attribution can be grouped broadly into five categories (Table 1) (EFSA, 2018; Mughini-Gras et al., 2018; Pires et al., 2009). Epidemiological surveys aim to investigate the causal relationship between the occurrence of health outcome and exposure to certain microbiological hazards. The most commonly used study designs are outbreak investigation, case-control or cohort studies. If relative risk estimates are obtained from those studies, then the population attributable fraction can be calculated when the proportion of exposed subjects is known. Microbiological studies and sporozoite-specific serological assay focus on cases only. Evidence synthesizing approaches including expert knowledge elicitation, systematic review in combination with techniques to summarize associations (e.g., meta-analysis). These approaches try to combine information from different studies or sources to summarize existing evidence. Finally, the quantitative microbiological risk assessment (QMRA), it collects all available data on different aspects together (e.g., contamination level of sources, consumption data, and dose-response relationship) to provide information on source attribution. In general, the choice of source attribution methods depends on many factors, such as the point of attribution along the food chain, the characteristics of the target pathogen, the availability and quality of data (Mughini-Gras et al., 2018).

Table 1. Different source attribution approaches for T. gondii, adapted from EFSA BIOHAZ Panel (2018).

Source attribution approach

1 Epidemiological studies

1.1 Outbreak investigations

1.2 Case-control studies and cohort studies

2 Microbiological studies

3 Sporozoite-specific serological assay

$4 \quad$ Evidence synthesizing approaches

4.1 Expert knowledge elicitation

4.2 Systematic review and meta-analysis

5 Quantitative microbiological risk assessment 
1 Epidemiological studies

\subsection{Outbreak investigations}

The primary goal of outbreak investigations is to find the source of infection to prevent additional cases. Highly specific details captured in an outbreak investigation can help identifying risk that cannot be examined in the routine evaluation of a single case. However, $T$. gondii outbreaks are rarely noticed and documented, because most of the infections are asymptomatic. Only a few acute toxoplasmosis outbreaks in humans have been reported worldwide (e.g., Smith, 1993; Bowie et al., 1997; Coutinho et al., 1982; Ekman et al., 2012; Pinto-Ferreira et al., 2019). Reported T. gondii outbreaks were mainly associated with water contaminated with oocysts due to the large number of people who could be infected from one source. The main limitation of outbreak investigations for source attribution is that they mainly focus on recent infections. In addition, the strain of the pathogen or specific sources associated with outbreaks might be different from sporadic cases.

\subsection{Case-control studies and cohort studies}

Several case-control studies focus on the attribution of the total disease burden to various sources have been published (e.g., Cook et al., 2000; Jones et al., 2009). In Europe, a case-control epidemiological study in pregnant women conducted in six large cities (i.e., Naples, Lausanne, Copenhagen, Oslo, Brussels, and Milan) showed that meat contributed $30-63 \%$ of $T$. gondii infection, although the importance of meat type differed depending on the countries. In contrast, $6-17 \%$ of infections could be attributed to soil contact (Cook et al., 2000). In the United States, the proportion of human cases that are food-borne (including vegetables) was estimated to be around 50\% based on serological data from 1999-2004 (Scallan et al., 2011). However, the definition of $T$. gondii infected cases is often based on serological test rather than incident cases, which means exposure may have long before measuring it. This may lead to misclassification of exposure. Besides, data are often generated from a relatively small number of participants, thus not representative of the target population. Therefore, a long-term prospective study (e.g., cohort study) is needed to identify and include enough incident cases to provide sufficient statistical power.

\section{Microbiological studies}

Microbiological approaches discussed here refers to studies based on statistical modelling of subtyping data from human cases and pathogen occurrences in selected animal, food and environmental sources (Pires et al., 2009). The principle is to match the subtypes of the human isolates with the most likely subtypes of isolates from different sources. This one-to-one matching method has been used for some major foodborne pathogens, for example Salmonella (Hald et al., 2004), Campylobacter (Mughini-Gras et al., 2016) and Escherichia coli (Mughini-Gras et 
al., 2019). This approach is in rapid development due to the increasing availability of whole genome sequencing data. However, it is less feasible for $T$. gondii than many other foodborne pathogens due to the limited genetic diversity in most geographical regions, except Central and South America (Rajendran et al., 2012; Su et al., 2012; Verma et al., 2015; Wang et al., 2013). Sensitive methods for distinguishing the subtypes are needed to reveal the transmission patterns of the parasite in the future (Ajzenberg et al., 2015). In addition, potential selection bias needs to be taken into account in this type of study for obtaining accurate estimates.

\section{Sporozoite-specific serological assay}

Recently, a serological assay has been developed to specifically detect oocystacquired infections, by measuring the antibody response to sporozoite specific antigens in recently infected individuals (humans, mice and pigs) (Hill et al., 2011). These antibodies are detectable during the first 6-8 months after infection, and will give an indication of oocyst transmitted sources on population level. The use of this test in selected populations has shown a high rate of oocyst acquired infections in the United States (78\%) and Chile (45\%) (Boyer et al., 2011). So far, no such study has been conducted in Europe (EFSA, 2018).

\section{Evidence synthesizing approaches}

\subsection{Expert knowledge elicitation}

Expert knowledge elicitation has been used successfully in a wide range of applications when primary data was limited or difficult to obtain (Butler et al., 2015; Van der Fels-Klerx et al., 2005). It generally consists of a preparation step (e.g., expert recruitment and training), elicitation session (e.g., online survey, or interview), and post-elicitation step (e.g., robustness and discrepancy analysis) (Cooke and Goossens 2000). Based on judgements of 72 experts, source attribution for eleven diarrheal diseases, seven other infectious diseases and one chemical (lead) were first estimated for 14 global subregions (Hald et al., 2016). The foodborne route was considered the most important transmission pathway for $T$. gondii by the experts, and a clear increasing tendency on the relative importance of soil route was observed in less developed subregions. Hoffmann et al. (2017) further analyzed the data and found that red meat (i.e., beef, small ruminants' meat and pork) were estimated to be the largest source of foodborne toxoplasmosis, accounting for $50 \%$ to $64 \%$ in all subregions. In addition, a large variation was also found for specific sources across subregions, e.g., vegetables were estimated to causes $21-23 \%$ of illnesses in European and South-East Asia subregions, which were larger than in other subregions (14-19\%). In the Netherlands, experts indicated that $56 \%$ of $T$. gondii infections are foodborne and $36 \%$ via environmental contamination (Havelaar et al., 2008). Expert knowledge elicitation is until now the most frequently applied approach for attributing $T$. 
gondii infection to major transmission routes of toxoplasmosis, however, it has the drawback that the data driving the knowledge of the experts are not specified (EFSA, 2018).

\subsection{Systematic review and meta-analysis}

Data from different sources may be useful in answering a specific research question by conducting a systematic review. Meta-analyses are a subset of systematic reviews, and can be considered as a helpful tool for pooling data from different sources in a structural way. In a systematic review and meta-analysis of 11 case-control studies, consumption of raw/undercooked meat, beef, and sheep meat were identified as three risk factors significantly associated with $T$. gondii infection (Belluco et al., 2017). However, risk factors do not necessarily give an indication of source attribution if there is hardly any exposure to the identified risk factor on population level.

\section{Quantitative microbial risk assessment}

QMRA is the application of principles of risk assessment to estimate the consequences from a planned or actual exposure to infectious microorganisms (Charles N. Haas, 2014). It is seen as a useful tool to rank the risk of human diseases through various pathways and to compare different intervention strategies. In performing a QMRA, the risk assessors aim to use the best available information to understand the nature of adverse effects from a microbial exposure to human populations. The process of QMRA development includes two phases: (1) problem formulation and (2) analysis. The problem formulation phase is essential for addressing the appropriate issue to meet the needs of the risk managers and stakeholders, for identifying the needed data and explaining assumptions at every step of the process. In the analysis phase, four steps are defined: (a) hazard identification aims to describe the human adverse effects associated with any particular hazard; (b) dose-response modelling aims to characterize the relationship between multiple doses exposed to humans and the incidence of health effect; (c) exposure assessment aims to determine the amount, duration, pathways of the hazard exposure; (d) risk characterization is used to integrate the knowledge from above-mentioned three steps to predict probability of an adverse outcome (Charles N. Haas, 2014).

The development of QMRA involves multidisciplinary knowledge from epidemiology, medicine, microbiology, food safety and risk assessment. To date, several QMRAs concerning the risk of meat-borne $T$. gondii infection to different populations have been published. The first quantitative risk assessment model (QMRA) focused on the relative atrribution of beef, pork and sheep products to human infection in the Netherlands (Opsteegh et al., 2011a). Afterwards, one quantitative model that focused on lamb (Guo et al., 2016b), three QMRAs that 
focused on the risk from pork (Condoleo et al., 2017; Crotta et al., 2017; Guo et al., 2017), and one that focussed on beef and pork (Belluco et al., 2018) were published. In addition, a probabilistic model to assess the exposure to T. gondii through fresh pork meat in England has been developed. However, these models only focused on meat-borne infections, and did not include transmission via oocysts. QMRA approaches for oocyst-borne infections are not reported. Subsequently, the relative importance of oocyst-borne $T$. gondii infection cannot be compared to results obtained from QMRA studies for meat-borne infection.

\section{MAIN OBJECTIVES OF THE THESIS}

T. gondii is one of the most well-studied parasites due to its medical importance in humans and great economic loss in livestock industries. Extensive studies have been performed all around the world trying to solve different problems. However, research questions remain, including the most cost-effective interventions to prevent infection, and to reduce the disease burden, the risk of infection in human, especially in risk groups and the relative attribution of different transmission routes to human infections.

To identify and prioritize successful prevention strategies, it is important to attribute the burden of toxoplasmosis to specific sources. QMRA is a useful tool to collect all available data on different aspects so that the data can be used for source attribution. In this thesis, we focused on the assessment of relative attribution of different sources (namely different meat products and soil) to human $T$. gondii infection in the Netherlands by using a quantitative microbial risk assessment approach. In addition, we implemented the QMRA model of meatborne $T$. gondii infection from the Netherlands for the mainland of China, where consumers have different consumption preferences and patterns.

The framework of this thesis is chronological with regards to the development of QMRA models. The thesis consists of the following parts:

\section{PART I MULTI-HOST TRANSMISSION}

In chapter 2 a systematic review on the transmission of $T$. gondii between different compartments using mathematical models is presented. It provided an overview of existing mathematical models and their assessments of different control strategies.

\section{PART II EPIDEMIOLOGICAL INSIGHTS}

In this section, seroprevalence of $T$. gondii in farm animals and pregnant women were analyzed and provided relevant input data for setting up QMRA models. 
In chapter 3 a cross-sectional study is presented estimating the seroprevalence and associated risk factors in indoor-housed Dutch dairy goat. A total of 1,664 goat sera were tested by using a commercial indirect ELISA, and the serological results were analyzed using binary mixture models. Potential risk factors were evaluated by comparing Poisson, negative binomial, and zero-inflated regression models.

In chapter 4 a systematic review and hierarchical meta-analysis is presented to determine and compare the regional distribution of $T$. gondii seroprevalence in pregnant women and meat-producing livestock in China. We analyzed the apparent and true seroprevalence by region using a novel Bayesian hierarchical model that allowed incorporating sensitivity and specificity of the applied serological assays.

\section{PART III QUANTITATIVE MICROBIAL RISK ASSESSMENTS}

In this section, the relative importance of the different types of meat to human infection in the Netherlands and the mainland of China was analyzed by QMRA. In addition, a QMRA model was set up for estimating the risk associated with $T$. gondii exposure via accidental soil ingestion from residential gardens in the Netherlands.

In chapter 5 the conceptual QMRA model of meat-borne $T$. gondii infection in the Netherlands as described previously by Opsteegh et al. (2011) was updated based on new insights in farm animals and an improved model was built based on recent T. gondii inactivation methods using salting. Literature data on the effect of salting on $T$. gondii viability were collected and used to fit a generalized linear mixed effects model. In addition to the new salting model, a lower concentration of bradyzoites in cattle, more specific heating profiles and more recent consumption data were also implemented in the QMRA model to estimate the number of new meat-borne $T$. gondii infections per year in the susceptible Dutch general population and among susceptible pregnant women.

In chapter 6 the first QMRA model of meat-borne $T$. gondii infection in the mainland of China is presented. Sensitivity and scenario analyses were carried out to identify influential parameters in the model. Moreover, the incidence rate of $T$. gondii in humans was additionally estimated by a deterministic susceptibleinfected (SI) model for validation purposes.

In chapter 7 a QMRA model was developed to estimate the risk associated with $T$. gondii exposure via soil intake as a proxy for oocyst ingestion in the Netherlands. A sensitive magnetic capture method for processing, recovery and detection of $T$. gondii oocysts in soil samples was modified and used for 166 soil samples from residential gardens. 
In chapter $\mathbf{8}$ an overall summary of the main results from this thesis is presented. In addition, the limitations of the studies and avenues for future research were discussed.

The present work has the following objectives:

1. To obtain an overview of existing mathematical models for transmission of $T$. gondii between different compartments (e.g., felines, environment, intermediate hosts, mechanical vectors), and to compare the prevention and control strategies investigated by the models;

2. To determine the seroprevalence of $T$. gondii infection in indoor-housed Dutch dairy goats and to identify the risk factors related to $T$. gondii seroprevalence;

3. To estimate the true prevalence of $T$. gondii in pregnant women and in the main meat-producing animals (i.e., pig, cattle, sheep, goat, chicken, duck, goose and donkey) from different regions of China;

4. To estimate the number of new meat-borne T. gondii infections per year in the susceptible Dutch general population and susceptible pregnant women with up-to-date input information, and to re-evaluate the effect of salting including all literature currently available;

5. To set up a QMRA model to estimate the relative attribution of different types of meat (i.e., pork, beef, lamb and mutton, chicken and duck) to human $T$. gondii infections in China;

6. To develop a magnetic capture method for detection of $T$. gondii oocysts in soil samples;

7. To build a QMRA model for estimating the risk associated with $T$. gondii exposure via accidental soil ingestion in the Netherlands. 


\section{REFERENCES}

Ajzenberg, D., Collinet, F., Aubert, D., Villena, I., Darde, M.L., French ToxoBs network group, Devillard, S., 2015. The rural-urban effect on spatial genetic structure of type II Toxoplasma gondii strains involved in human congenital toxoplasmosis, France, 2002-2009. Infect Genet Evol 36, 511-516.

Belluco, S., Simonato, G., Mancin, M., Pietrobelli, M., Ricci, A., 2017. Toxoplasma gondii infection and food consumption: A systematic review and meta-analysis of case-controlled studies. Crit Rev Food Sci Nutr, 1-12.

Bonačić Marinović, A.A., Opsteegh, M., Deng, H., Suijkerbuijk, A.W.M., van Gils, P.F., van der Giessen, J., 2019. Prospects of toxoplasmosis control by cat vaccination. Epidemics 30, 100380.

Boyer, K., Hill, D., Mui, E., Wroblewski, K., Karrison, T., Dubey, J.P., Sautter, M., Noble, A.G., Withers, S., Swisher, C., Heydemann, P., Hosten, T., Babiarz, J., Lee, D., Meier, P., McLeod, R., 2011. Unrecognized ingestion of Toxoplasma gondii oocysts leads to congenital toxoplasmosis and causes epidemics in North America. Clin Infect Dis 53, 1081-1089.

Burrells, A., Benavides, J., Cantón, G. et al. Vaccination of pigs with the $\$ 48$ strain of Toxoplasma gondii safer meat for human consumption. Vet Res 46, 47 (2015). https://doi.org/10.1186/s13567-015-0177-0

Butler, A.J., Thomas, M.K., Pintar, K.D., 2015. Expert elicitation as a means to attribute 28 enteric pathogens to foodborne, waterborne, animal contact, and person-to-person transmission routes in Canada. Foodborne Pathog Dis 12, 335-344.

Charles N. Haas, J.B.R., Charles P. Gerba, 2014. Quantitative Microbial Risk Assessment, 2nd Edition.

Cook, A.J., Gilbert, R.E., Buffolano, W., Zufferey, J., Petersen, E., Jenum, P.A., Foulon, W., Semprini, A.E., Dunn, D.T., 2000. Sources of toxoplasma infection in pregnant women: European multicentre case-control study. European Research Network on Congenital Toxoplasmosis. Bmj 321, 142-147.

Cooke, R.M., Goossens , L.H.J., 2000. Procedures guide for structural expert judgement in accident consequence modelling. Radiation Protection Dosimetry 90, 303-309.

Dubey, J.P., 1995. Duration of immunity to shedding of Toxoplasma gondii oocysts by cats. J Parasitol 81, 410-415. Dubey, J.P., 1998. Advances in the life cycle of Toxoplasma gondii. Int J Parasitol 28, 1019-1024.

Dubey, J.P., 2008. The history of Toxoplasma gondii--the first 100 years. J Eukaryot Microbiol 55, 467-475.

Dubey, J.P., Miller, N.L., Frenkel, J.K., 1970. The Toxoplasma gondii oocyst from cat feces. J Exp Med 132, 636-662.

Dunn, D., Wallon, M., Peyron, F., Petersen, E., Peckham, C., Gilbert, R., 1999. Mother-to-child transmission of toxoplasmosis: risk estimates for clinical counselling. Lancet 353, 1829-1833.

ECDC, 2019. Introduction to the annual epidemiological report. In: ECDC. Annual epidemiological report for 2017, Stockholm.

EFSA, 2018. Public health risks associated with food-borne parasites. EFSA Journal 16, e05495.

Katzer F, Canton G, Burrells A, et al. Immunization of lambs with the S48 strain of Toxoplasma gondii reduces tissue cyst burden following oral challenge with a complete strain of the parasite. Vet Parasitol. 2014;205(1-2):46-56. doi:10.1016/j.vetpar.2014.07.003

Frenkel, J.K., Dubey, J.P., 1973. Effects of freezing on the viability of toxoplasma oocysts. J Parasitol 59, 587-588.

Frenkel, J.K., Ruiz, A., Chinchilla, M., 1975. Soil survival of toxoplasma oocysts in Kansas and Costa Rica. Am J Trop Med Hyg 24, 439-443.

Gilbert, R.E., Stanford, M.R., 2000. Is ocular toxoplasmosis caused by prenatal or postnatal infection? Br J Ophthalmol 84, 224-226.

Hald, T., Aspinall, W., Devleesschauwer, B., Cooke, R., Corrigan, T., Havelaar, A.H., Gibb, H.J., Torgerson, P.R., Kirk, M.D., Angulo, F.J., Lake, R.J., Speybroeck, N., Hoffmann, S., 2016. World Health Organization estimates of the relative contributions of food to the burden of disease due to selected foodborne hazards: $A$ structured expert elicitation. PLoS One 11, e0145839.

Hald, T., Vose, D., Wegener, H.C., Koupeev, T., 2004. A Bayesian approach to quantify the contribution of animalfood sources to human salmonellosis. Risk Anal 24, 255-269.

Halonen, S.K., Weiss, L.M., 2013. Toxoplasmosis. Handb Clin Neurol 114, 125-145.

Havelaar, A.H., Galindo, A.V., Kurowicka, D., Cooke, R.M., 2008. Attribution of foodborne pathogens using structured expert elicitation. Foodborne Pathog Dis 5, 649-659.

Havelaar, A.H., Haagsma, J.A., Mangen, M.J., Kemmeren, J.M., Verhoef, L.P., Vijgen, S.M., Wilson, M., Friesema, I.H., Kortbeek, L.M., van Duynhoven, Y.T., van Pelt, W., 2012. Disease burden of foodborne pathogens in the Netherlands, 2009. Int J Food Microbiol 156, 231-238.

Havelaar, A.H., Kirk, M.D., Torgerson, P.R., Gibb, H.J., Hald, T., Lake, R.J., Praet, N., Bellinger, D.C., de Silva, N.R., Gargouri, N., Speybroeck, N., Cawthorne, A., Mathers, C., Stein, C., Angulo, F.J., Devleesschauwer, B., 2015. World Health Organization global estimates and regional comparisons of the burden of foodborne disease in 2010. PLoS Med 12, e1001923. 
Hill, D., Coss, C., Dubey, J.P., Wroblewski, K., Sautter, M., Hosten, T., Muñoz-Zanzi, C., Mui, E., Withers, S., Boyer, K., Hermes, G., Coyne, J., Jagdis, F., Burnett, A., McLeod, P., Morton, H., Robinson, D., McLeod, R., 2011. Identification of a sporozoite-specific antigen from Toxoplasma gondii. J Parasitol 97, 328-337.

Hill, D., Dubey, J.P., 2002. Toxoplasma gondii: transmission, diagnosis and prevention. Clin Microbiol Infect 8, 634640.

Hoffmann, S., Devleesschauwer, B., Aspinall, W., Cooke, R., Corrigan, T., Havelaar, A., Angulo, F., Gibb, H., Kirk, M., Lake, R., Speybroeck, N., Torgerson, P., Hald, T., 2017. Attribution of global foodborne disease to specific foods: findings from a World Health Organization structured expert elicitation. PLoS One 12, e0183641.

Jones, J.L., Dargelas, V., Roberts, J., Press, C., Remington, J.S., Montoya, J.G., 2009. Risk factors for Toxoplasma gondii infection in the United States. Clin Infect Dis 49, 878-884.

Kniel, K.E., Lindsay, D.S., Sumner, S.S., Hackney, C.R., Pierson, M.D., Dubey, J.P., 2002. Examination of attachment and survival of Toxoplasma gondii oocysts on raspberries and blueberries. J Parasitol 88, 790-793.

Kortbeek, L.M., Hofhuis, A., Nijhuis, C.D., Havelaar, A.H., 2009. Congenital toxoplasmosis and DALYs in the Netherlands. Mem Inst Oswaldo Cruz 104, 370-373.

Lambooij, M.S., Veldwijk, J., van Gils, P., Mangen, M.-J.J., Over, E., Suijkerbuijk, A., Polder, J., de Wit, G.A., Opsteegh, M., 2019. Consumers' preferences for freezing of meat to prevent toxoplasmosis- A stated preference approach. Meat Sci 149, 1-8.

Lelu, M., Villena, I., Darde, M.L., Aubert, D., Geers, R., Dupuis, E., Marnef, F., Poulle, M.L., Gotteland, C., Dumetre, A., Gilot-Fromont, E., 2012. Quantitative estimation of the viability of Toxoplasma gondii oocysts in soil. Appl Environ Microbiol 78, 5127-5132.

Lindsay, D.S., Dubey, J.P., 2009. Long-term survival of Toxoplasma gondii sporulated oocysts in seawater. J Parasitol 95, 1019-1020.

Martorelli Di Genova, B., Wilson, S.K., Dubey, J.P., Knoll, L.J., 2019. Intestinal delta-6-desaturase activity determines host range for Toxoplasma sexual reproduction. PLOS Biology 17, e3000364.

Miller, N.L., Frenkel, J.K., Dubey, J.P., 1972. Oral infections with Toxoplasma cysts and oocysts in felines, other mammals, and in birds. J Parasitol 58, 928-937.

Mirza Alizadeh, A., Jazaeri, S., Shemshadi, B., Hashempour-Baltork, F., Sarlak, Z., Pilevar, Z., Hosseini, H., 2018. A review on inactivation methods of Toxoplasma gondii in foods. Pathog Glob Health 112, 306-319.

Montoya, J.G., Liesenfeld, O., 2004. Toxoplasmosis. Lancet 363, 1965-1976.

Mughini-Gras, L., Dorado-García, A., van Duijkeren, E., van den Bunt, G., Dierikx, C.M., Bonten, M.J.M., Bootsma, M.C.J., Schmitt, H., Hald, T., Evers, E.G., de Koeijer, A., van Pelt, W., Franz, E., Mevius, D.J., Heederik, D.J.J., 2019. Attributable sources of community-acquired carriage of Escherichia coli containing $\beta$-lactam antibiotic resistance genes: a population-based modelling study. The Lancet Planetary Health 3, e357-e369.

Mughini-Gras, L., Kooh, P., Augustin, J.-C., David, J., Fravalo, P., Guillier, L., Jourdan-Da-Silva, N., Thébault, A., Sanaa, M., Watier, L., Anses Working Group on Source Attribution of Foodborne, D., 2018. Source attribution of foodborne diseases: potentialities, hurdles, and future expectations. Frontiers in Microbiology 9, 1983-1983.

Mughini-Gras, L., Penny, C., Ragimbeau, C., Schets, F.M., Blaak, H., Duim, B., Wagenaar, J.A., de Boer, A., Cauchie, H.M., Mossong, J., van Pelt, W., 2016. Quantifying potential sources of surface water contamination with Campylobacter jejuni and Campylobacter coli. Water Res 101, 36-45.

Nissen, J., Jokelainen, P., Stensvold, C.R., Trevisan, C., Fuchs, J., Burgdorf, K.S., Nielsen, H.V., Pires, S.M., 2017. The disease burden of congenital toxoplasmosis in Denmark, 2014. PLoS One 12, e0178282.

Opsteegh, M., Kortbeek, T.M., Havelaar, A.H., van der Giessen, J.W., 2015. Intervention strategies to reduce human Toxoplasma gondii disease burden. Clin Infect Dis 60, 101-107.

Opsteegh, M., Maas, M., Schares, G., Van der Giessen, J., on behalf of the consortium, 2016a. Relationship between seroprevalence in the main livestock species and presence of Toxoplasma gondii in meat (GP/EFSA/BIOHAZ/2013/01). An Extensive Literature Review. Final Report. EFSA.

Opsteegh, M., Schares, G., Blagac, R., van der Giessen, J., on behalf of the consortium, 2016b. Experimental studies on Toxoplasma gondii in the main livestock species (GP/EFSA/BIOHAZ/2013/01) Final report. EFSA.

Pappas, G., Roussos, N., Falagas, M.E., 2009. Toxoplasmosis snapshots: global status of Toxoplasma gondii seroprevalence and implications for pregnancy and congenital toxoplasmosis. Int J Parasitol 39, 13851394.

Pijnacker, R., Friesema, I.H., Mughini Gras, L., Lagerweij, G.R., van Pelt, W., Franz, E., 2019. Disease 
burden of food-related pathogens in the Netherlands, 2018. RIVM.

Pires, S.M., Evers, E.G., van Pelt, W., Ayers, T., Scallan, E., Angulo, F.J., Havelaar, A., Hald, T., Med-Vet-Net Workpackage 28 Working, G., 2009. Attributing the human disease burden of foodborne infections to specific sources. Foodborne Pathog Dis 6, 417-424.

Pinto-Ferreira F, Caldart E, Pasquali A, Mitsuka-Breganó R, Freire R, Navarro I. Patterns of transmission and sources of infection in outbreaks of human toxoplasmosis. Emerg Infect Dis. 2019;25(12):2177-2182. https://dx.doi.org/10.3201/eid2512.181565

Rajendran, C., Su, C., Dubey, J.P., 2012. Molecular genotyping of Toxoplasma gondii from Central and South America revealed high diversity within and between populations. Infect Genet Evol 12, 359-368.

Robert-Gangneux, F., Darde, M.L., 2012. Epidemiology of and diagnostic strategies for toxoplasmosis. Clin Microbiol Rev 25, 264-296.

Rougier, S., Montoya, J.G., Peyron, F., 2017. Lifelong Persistence of Toxoplasma Cysts: A Questionable Dogma?: (Trends in Parasitology 33, 93-101; 2017). Trends Parasitol 33, 414.

Scallan, E., Hoekstra, R.M., Angulo, F.J., Tauxe, R.V., Widdowson, M.A., Roy, S.L., Jones, J.L., Griffin, P.M., 2011. Foodborne illness acquired in the United States--major pathogens. Emerg Infect Dis 17, 7-15.

Schmidt, D.R., Hogh, B., Andersen, O., Fuchs, J., Fledelius, H., Petersen, E., 2006. The national neonatal screening programme for congenital toxoplasmosis in Denmark: results from the initial four years, 1999-2002. Arch Dis Child 91, 661-665.

Su, C., Khan, A., Zhou, P., Majumdar, D., Ajzenberg, D., Dardé, M.-L., Zhu, X.-Q., Ajioka, J.W., Rosenthal, B.M., Dubey, J.P., Sibley, L.D., 2012. Globally diverse Toxoplasma gondii isolates comprise six major clades originating from a small number of distinct ancestral lineages. Proceedings of the National Academy of Sciences 109, 5844-5849.

Suijkerbuijk, A.W.M., Over, E.A.B., Opsteegh, M., Deng, H., Gils, P.F.V., Bonacic Marinovic, A.A., Lambooij, M., Polder, J.J., Feenstra, T.L., Giessen, J., Wit, G.A., Mangen, M.J., 2019. A social cost-benefit analysis of two One Health interventions to prevent toxoplasmosis. PLoS One 14, e0216615.

Smith J.L. Documented outbreaks of toxoplasmosis: transmission of Toxoplasma gondii to Humans. J Food Prot. 1993;56(7):630-639. doi:10.4315/0362-028X-56.7.630

Tenter, A.M., Heckeroth, A.R., Weiss, L.M., 2000. Toxoplasma gondii: from animals to humans. Int J Parasitol 30, 1217-1258.

Thiebaut, R., Leproust, S., Chene, G., Gilbert, R., 2007. Effectiveness of prenatal treatment for congenital toxoplasmosis: a meta-analysis of individual patients' data. Lancet 369, 115-122.

Torgerson, P.R., Devleesschauwer, B., Praet, N., Speybroeck, N., Willingham, A.L., Kasuga, F., Rokni, M.B., Zhou, X.N., Fevre, E.M., Sripa, B., Gargouri, N., Furst, T., Budke, C.M., Carabin, H., Kirk, M.D., Angulo, F.J., Havelaar, A., de Silva, N., 2015. World Health Organization estimates of the global and regional disease burden of 11 foodborne parasitic diseases, 2010: A data synthesis. PLoS Med 12, e1001920.

Torgerson, P.R., Mastroiacovo, P., 2013. The global burden of congenital toxoplasmosis: a systematic review. Bull World Health Organ 91, 501-508.

Turner, M., Lenhart, S., Rosenthal, B., Zhao, X., 2013. Modeling effective transmission pathways and control of the world's most successful parasite. Theoretical Population Biology 86, 50-61.

Van der Fels-Klerx, H.J., Cooke, R.M., Nauta, M.N., Goossens, L.H., Havelaar, A.H., 2005. A structured expert judgment study for a model of Campylobacter transmission during broiler-chicken processing. Risk Anal 25, 109-124.

Verma, S.K., Ajzenberg, D., Rivera-Sanchez, A., Su, C., Dubey, J.P., 2015. Genetic characterization of Toxoplasma gondii isolates from Portugal, Austria and Israel reveals higher genetic variability within the type II lineage. Parasitology 142, 948-957.

Voedingscentrum, 2020. Toxoplasmose. https://www.voedingscentrum.nl/encyclopedie/toxoplasmose.aspx

Wang, L., Chen, H., Liu, D., Huo, X., Gao, J., Song, X., Xu, X., Huang, K., Liu, W., Wang, Y., Lu, F., Lun, 


\section{6 - CHAPTER 1}

Z.R., Luo, Q., Wang, X., Shen, J., 2013. Genotypes and mouse virulence of Toxoplasma gondii isolates from animals and humans in China. PLoS One 8, e53483. 


\section{Chapter}

\section{Mathematical modelling of Toxoplasma gondii transmission: A systematic review}

Huifang Deng ${ }^{1}$, Rachel Cummins ${ }^{1}$, Gereon Schares ${ }^{2}$, Chiara Trevisan ${ }^{3}$, Heidi Enemark$^{4}$, Helga Waap ${ }^{5}$, Jelena Srbljanovic ${ }^{6}$, Olgica Djurkovic-Djakovic ${ }^{7}$, Sara Monteiro Pires ${ }^{8}$, Joke W. B. van der Giessen ${ }^{1}$, Marieke Opsteegh ${ }^{1}$

Authors' affiliations:

${ }^{1}$ Centre for Infectious Disease Control - Zoonoses and Environmental Microbiology, National Institute for Public Health and the Environment, Bilthoven, the Netherlands

${ }^{2}$ Institute of Epidemiology, Federal Research Institute for Animal Health, Insel Riems, Germany

${ }^{3}$ Department of Biomedical Sciences, Institute of Tropical Medicine, Antwerp, Belgium

${ }^{4}$ Department of Animal Health and Food Safety, Norwegian Veterinary Institute, Oslo, Norway

${ }^{5}$ Laboratório de Parasitologia, Instituto Nacional de Investigação Agrária e Veterinária, Oeiras, Portugal

${ }^{6}$ Centre of Excellence for Food- and Vector-borne Zoonoses, National Reference Laboratory for Toxoplasmosis, Institute for Medical Research, University of Belgrade, Belgrade, Serbia

${ }^{7}$ National Food Institute, Technical University of Denmark, Lyngby, Denmark 


\begin{abstract}
Background: Toxoplasma gondii is a ubiquitous protozoan parasite that can infect virtually all warm-blooded animals. It is the causative agent of toxoplasmosis, a significant public health issue worldwide. Mathematical models are useful to study the transmission dynamics of $T$. gondii infection in different settings, and may be used to compare the effectiveness of prevention measures.
\end{abstract}

Methods: To obtain an overview of existing mathematical models for transmission of $T$. gondii, a systematic review was undertaken. The review was conducted according to an a priori protocol. Specific search terms were developed and used in the search of three databases (Scopus, PubMed, and Embase).

Results: In total, 484 unique records were retrieved from the systematic search. Among them, 15 studies that used mathematical models to study the transmission of $T$. gondii. These studies were categorized into four groups based on the primary aims: dynamics of transmission $(n=8)$, intervention $(n=5)$, spatial distribution $(n=1)$, and outbreak investigation $(n=1)$.

Conclusions: Considering the high disease burden caused by $T$. gondii, studies using mathematical models to understand the transmission dynamics of this parasite and to evaluate the effectiveness of intervention measures were scarce. This systematic review provides an overview of existing mathematical models and identifies the data gaps for model building. The results from this study can be helpful for further development of mathematical models and improved understand of the transmission dynamics of $T$. gondii infection. 


\section{Introduction}

Toxoplasma gondii is one of the most widespread zoonotic parasites in the world, that can infect virtually all warm-blooded vertebrates. T. gondii is the causative agent of toxoplasmosis, a potentially serious disease in humans leading to a large public health burden and causing large economic losses in the livestock industry due to abortion and stillbirth (Tenter et al., 2000).

T. gondii has a complex life cycle functioning in a prey-predator system (Fig. 1). It involves three infectious stages (rapidly dividing invasive tachyzoites in all the nucleated cells, slowly dividing bradyzoites in tissue cysts, and sporozoites in oocysts), sexual and asexual reproduction, and multiple hosts (Dubey, 1998). As the only definitive host of the parasite, members of the felidae family play the most important role in $T$. gondii transmission. Cats can become infected by ingesting any of the three infectious stages of T. gondii (Miller et al., 1972), and following sexual reproduction in their intestines they can excrete millions of unsporulated oocysts into the environment through faeces (Dubey et al., 1970). This may continue for up to about 20 days (Dubey, 1995). Oocysts sporulate within a few days in the environment and can remain infectious for more than a year in soil or water under ambient conditions. A wide range of warm-blooded animals can get infected by ingestion of sporulated oocysts from contaminated environments and serve as the intermediate hosts of the parasite (Dubey, 1998). After ingestion of oocysts by an intermediate host, sporozoites are liberated in the intestine of the host where they invade enterocytes and differentiate into tachyzoites. Tachyzoites are disseminated rapidly via the blood stream to all tissues, and are able to infect all nucleated cells. Immune pressure triggers the conversion of tachyzoites into bradyzoites in tissue cysts, which form 7-10 days after infection and remain viable in multiple organs, including the central nervous system tissue and muscles (including the heart). It is generally believed that cysts may remain viable for the life of the host, triggering life-long immunity, but this concept has recently been challenged (Rougier et al., 2017). If a definitive host ingests an infected intermediate host (e.g., rodents or birds), then the cycle of T. gondii is completed. Moreover, T. gondii can be transmitted between intermediate hosts via carnivory, e.g., when people consume undercooked meat containing tissue cysts (RobertGangneux and Darde, 2012). In addition, T. gondii may be transmitted vertically; if primary infection occurs during pregnancy, the tachyzoites can cross the placenta and infect the fetus, leading to congenital toxoplasmosis (CT) (Dunn et al., 1999). 


\section{FOODBORNE TRANSMISSION PATHWAYS FOR TOXOPLASMA GONDII}

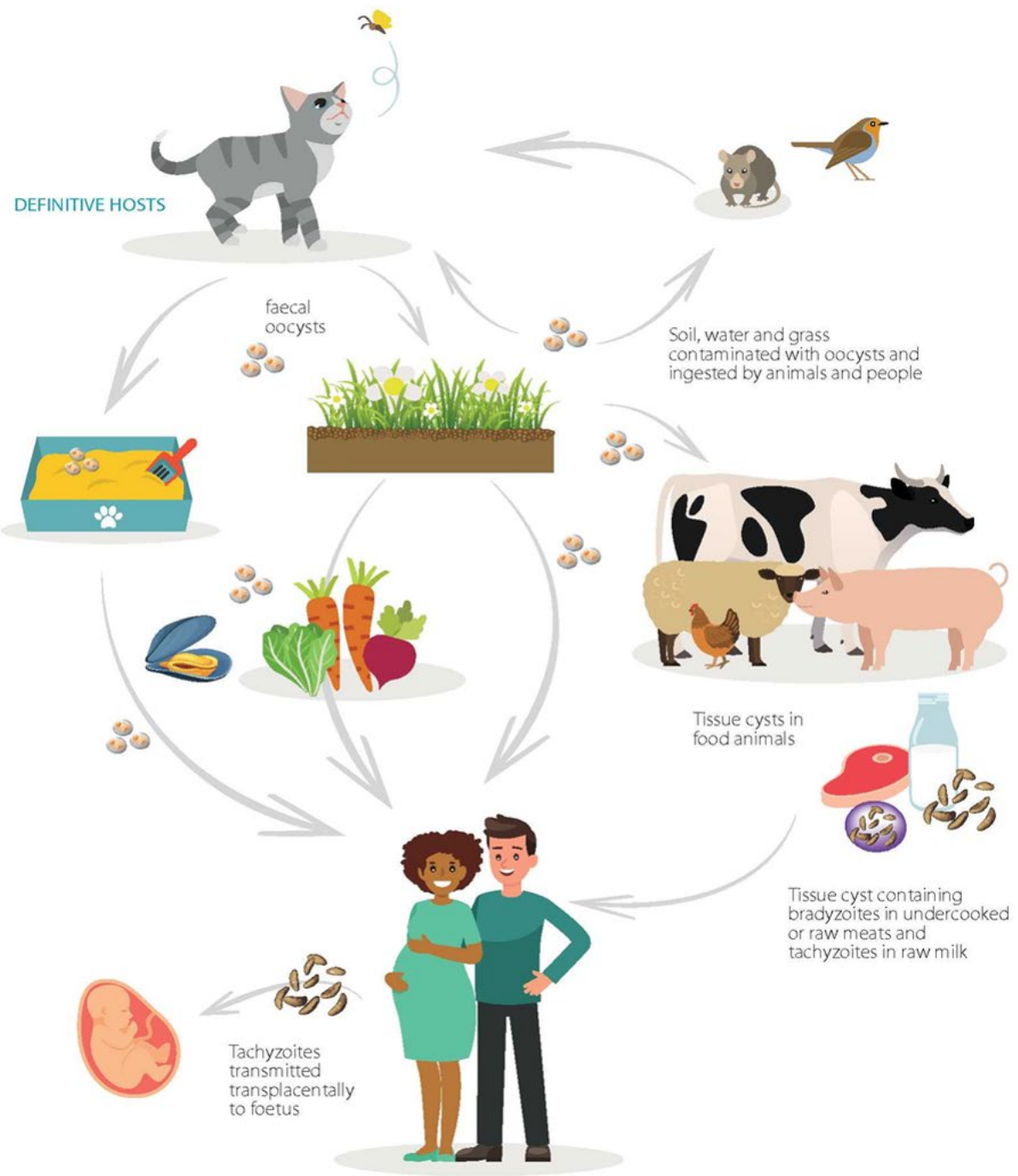

LEGEND 30 0ocysts (3) Tachyzoites Bradyzoite

Figure 1. Life cycle of Toxoplasma gondii, adapted from EFSA BIOHAZ Panel (2018). 
The bulk of the burden of disease associated with human $T$. gondii infection is actually due to CT. In 2013, the annual global incidence of CT was estimated at 1.5 cases per 1,000 live births, and the burden of CT was estimated to be 1.2 million DALYs (95\% Cl: 0.76-1.90) (Torgerson and Mastroiacovo, 2013). At the European level, $T$. gondii ranked second out of 24 food-borne parasites in a multicriteriabased ranking which considered public health, trade, and socio-economic impact (Bouwknegt et al., 2018). Despite the high disease burden of $T$. gondii infection at both population and individual level, toxoplasmosis is considered a neglected parasitic infection in the United States (Jones et al., 2014). In Europe, only a few countries (Austria, Belgium, France, Portugal, Slovakia and Slovenia) have active surveillance of congenital cases by mandatory screening of pregnant women, which show that relatively little attention has been devoted to its surveillance, prevention and treatment (DGS, 2013; ECDC, 2019; Peyron et al., 2017). Several intervention strategies have been proposed to reduce the disease burden of human infection (e.g., prenatal/neonatal screening and treatment, health education of pregnant women/general population, freezing of meat destined for raw or undercooked consumption, vaccination of cats, and biosecurity measures for food animals) (Opsteegh et al., 2015). However, except for health education and screening of pregnant women and other risk groups, additional intervention measures targeting the sources of infection are generally not in place because it is difficult to evaluate the balance between costs and benefits (Binquet et al., 2019; Bobić et al., 2019; Stillwaggon et al., 2011).

Models use mathematical language to describe the behaviour of a system and are useful to study the transmission dynamics of infectious diseases in different settings. They help better understand the transmission of a pathogen and can be used to validate, compare and optimise the preventive measures. For example, compartmental models, where the total population is divided into different compartments, are widely used in epidemiological studies to investigate the spread of infectious disease at the population level (Brauer et al., 2008). A simple and widely used compartmental model is the SIR model, which consists of three sub-populations, representing the number of susceptible (S), infected (I) and recovered $(\mathrm{R})$ individuals in each compartment at a particular time. The mathematical quantity of major interest is the basic reproduction number $\left(R_{0}\right)$, i.e., the average number of secondary cases generated by a typical infected individual introduced into a completely susceptible population (Dietz, 1993). Typically, its value determines whether an infectious disease can spread through a population or not. If $R_{0}$ is less than one, the disease will eventually die out, i.e., the population reaches a disease-free equilibrium. On the other hand, if the basic reproductive number is larger than one, the disease will be able to spread and remain in the population. However, T. gondii is not only transmitted between definitive hosts, but can also be transmitted via intermediate hosts and has a reservoir in the 
environment. Therefore, a SIR-model does not capture all compartments important in the transmission of $T$. gondii infection. Moreover, although cats excrete oocysts temporarily and move from the I to the R compartment regarding oocyst excretion, both cats and intermediate hosts will harbour tissue cysts potentially for life and remain infectious via carnivory, therefore an $R$ compartment is not applicable for the tissue cyst route.

The aim of this study was to provide an overview of existing mathematical models on the transmission of $T$. gondii between different compartments (e.g., felines, environment, intermediate hosts, mechanical vectors) and analyse model structures and outcomes by performing a systematic review. In addition to a comparison of the models, we also aimed to describe and compare the prevention and control strategies investigated by the models.

\section{Methods}

\subsection{Search strategy}

A systematic review following a predefined protocol was performed and reported based on PRISMA guidelines (www.prisma-statement.org). A search for eligible studies was carried out using three bibliographic databases: Scopus, PubMed, and Embase. For the initial identification of relevant studies we considered the development of specific search terms on the following key subjects: (1) T. gondii is the pathogen of interest; (2) it includes mathematical models; (3) the underlying model to the study of $T$. gondii transmission is mechanistic in its approach; (4) transmission of $T$. gondii between any of the compartments (i.e., feline definitive host, animal intermediate hosts (prey animals or livestock/wildlife for human consumption), humans, environment (e.g., soil and water), oocyst-contaminated produce or shellfish, and mechanical vectors (e.g., flies and worms)) or climatic conditions that influence oocyst dispersion and survival is described.

The databases were searched using keywords (with the use of Medical Subject Heading (MeSH) or Emtree terms used in PubMed and EMBASE respectively) associated with the Boolean operators AND/OR. Different combinations were tailored for each database in order to narrow the number of results retrieved but at the same time maximizing the number of relevant studies. The search language was restricted to English. There were no restrictions in study year or geographical location. Grey literature was not searched for. Retrieved records were imported in EndNote and duplicates were removed. The search strategy was validated for reliability, using a subset of publications already identified as relevant to the objective (Table 1). The final search (Table 2) was performed on January $13^{\text {th }}, 2020$. 


\subsection{Selection criteria}

Studies retrieved from the databases were assessed against the inclusion and exclusion criteria for relevance and eligibility. Before examination of all retrieved papers, publications that clearly did not fit the criteria described under section 2.1 (e.g., reviews, letter to editor) were excluded by two authors (pre-title/abstract screening), then all the remaining publications were assigned to all authors for screening. The screening was performed in two stages. First, a title and abstract screening was performed for relevance. The inclusion criteria for this stage were paraphrased into the following questions: (1) is an abstract available? (yes/no); (2) is $T$. gondii studied in the publication? (yes/no/unclear-full text required); (3) does the title/abstract suggest that a mathematical model is used to study transmission? (yes/no/unclear-full text required). All unique records were divided among all researchers, and were assessed by two different researchers at both title/abstract stage and full text stage. A third reviewer was consulted if a publication had contradicting results. If the first reviewer considered a record relevant or unclear, it was then included in the full-text screening. When the reviewer did not consider the record relevant, the record was screened by the second reviewer. If the second reviewer considered the record relevant or unclear it was included in the full-text screening, if the second reviewer agreed that the record was not relevant, the record was placed in the list of non-relevant records. If no abstract was available or any of the inclusion criteria mentioned above could not be properly evaluated based on title and abstract alone, the eligibility of those studies was evaluated based on the full text. Second, a full-text screening was performed on the publications selected in the first stage. Additional exclusion criteria applied in this phase included: (1) full-text could not be obtained within two weeks after the selection for full-text screening was completed for all records; (2) publication contains only duplicated data; (3) purely descriptive or statistical models that seek to fit data without consideration of underlying biological mechanisms (e.g., risk factor analysis); (4) mother to child transmission; (5) quantitative microbial risk assessment studies (they were included in another systematic review concerning source attribution of $T$. gondii infection). The screening was performed using Epilnfo (CDC, version 7.1.5). A PRISMA flowchart was used to summarize all stages of the paper selection process.

\subsection{Data extraction}

Data were extracted from all eligible papers using a predefined electronic form containing the following components: (1) the characteristics of the included studies (reference, published year, title); (2) location (where the study population was located); (3) model objectives (e.g., dynamics of transmission and evaluation of intervention strategies); (4) transmission routes of $T$. gondii; (5) model setting (e.g., urban and farm); (6) type of model (e.g., compartment model and agent- 
based model); (7) model outputs (e.g., environmental contamination rate, T. gondii prevalence, and $R_{0}$ ).

\section{Results}

The systematic search provided 484 records. All articles already identified as relevant to the objective a priori (Table 1) were also retrieved in the final search strategy. Forty-eight of the available records did not fit the inclusion criteria and were excluded based on title before title and abstract screening. The entire list of 436 records can be found in the supplementary material. After the title and abstract screening, 63 studies were selected for full-text screening. Of the 63 studies, 15 met the inclusion criteria after full-text screening and were included in the systematic review (Fig. 2).

Table 1. Articles used to validate the search query.

\begin{tabular}{ll}
\hline References & DOI \\
\hline Arenas et al., 2010 & $10.1016 / \mathrm{j} . t p b .2010 .03 .005$ \\
Gonzalez-Parra et al., 2009 & $10.1016 /$ j.camwa.2008.09.012 \\
Gotteland et al., 2014b & $10.1186 / 1476-072 X-13-45$ \\
Jiang et al., 2012 & $10.1016 /$ j.jtbi.2011.10.006 \\
Lélu et al., 2010 & $10.1016 /$ j.tpb.2010.05.005 \\
Turner et al., 2013 & $10.1016 /$ j.tpb.2013.04.001 \\
\hline
\end{tabular}

All included studies were published between 2002 and 2020, except one, which was published in 1982. Results were presented in four different categories: dynamics of transmission $(n=8)$, intervention $(n=5)$, spatial distribution $(n=1)$ and outbreak investigation $(n=1)$. However, one paper had components that fit more than one category (e.g., the spatial distribution and the dynamics of transmission category), it was only included in the spatial distribution category.

\subsection{Dynamics of transmission}

Of the selected papers, eight were classified as transmission dynamic modelling. These models were compared based on the compartments that were incorporated in the models (e.g., cats, mice, human and environment).

\subsubsection{Cat-human transmission}

Three papers focused on cat-human transmission (Ferreira et al., 2017; GonzálezParra et al., 2009; Yongzhen et al., 2018). González-Parra et al. (2009) developed a compartment model for exploring the dynamics of $T$. gondii transmission to humans (SIC-type: susceptible, infected, controlled (people in treatment)) with cat 
(SI-type: susceptible, infected) as a vector of transmission. These two populations were linked with the assumption that both humans and cats get infected with $T$. gondii only through contact with infected cats. In the model, the human and feline population sizes were set as constant by assuming birth rate equal to death rate. Vertical transmission in both populations was considered in the proposed system.

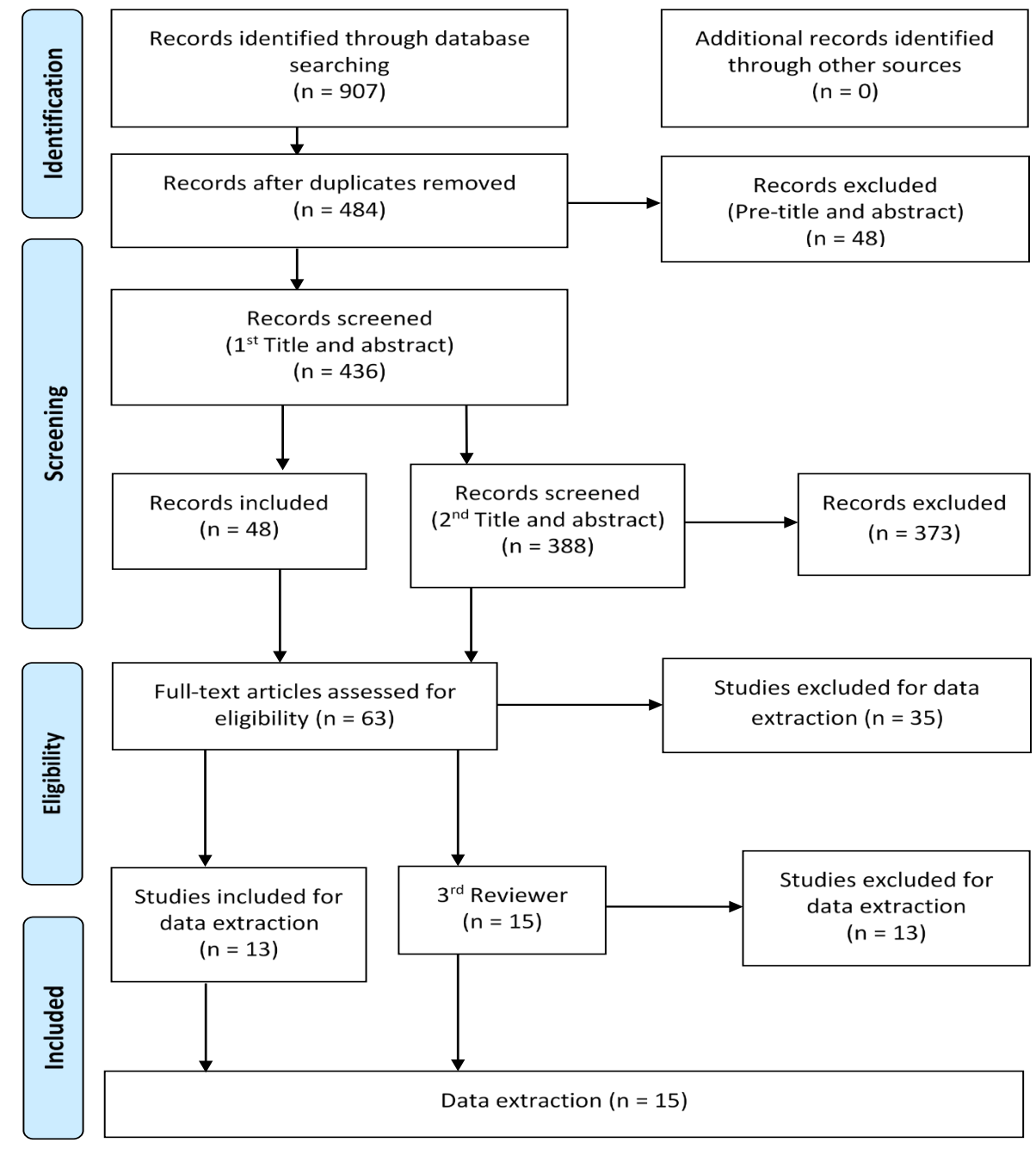

Figure 2. PRISMA flow diagram: search steps and selection of relevant studies on mathematical modelling of Toxoplasma gondii transmission. 
In the human population, vertical transmission was assumed with a probability of one, whereas a cat born from an infected cat had a probability $p_{\mathrm{c}}$ of not being infected. Scenario analysis concerning the $\mathrm{R}_{0}$ and vertical transmission of $T$. gondii in cats revealed that the $R_{0}$ in cats completely controls the dynamics of the infection and when a high vertical transmission in a cat population is assumed, the endemic equilibrium point has a higher proportion of infected cats and infected humans at the steady state. Note that infectiousness (in this case oocyst excretion) of vertically infected cats is questionable, and to the best of our knowledge oocyst excretion by vertically infected was not reported yet.

Ferreira et al. (2017) and Yongzhen et al. (2018) extended the model of GonzálezParra et al. (2009) to describe the transmission of $T$. gondii between cat and human populations. Both models assumed that horizontal transmission of the parasite to humans and cats was only through contact with infected cats. Ferreira et al. (2017) extended the model with a spatial version and similar to González-Parra et al. (2009), they showed that the $R_{0}$ depends on the contact rate of susceptible cats with infected cats, the birth/immigration rate of cats and the probability of effective infectious contact among cats. In the study by Yongzhen et al. (2018), vertical transmission was considered (both cats and humans born from an infected mother had probabilities ( $p_{1}$ and $p_{2}$ ) of not being infected), and the human population size was a variable. They showed that whether the disease will die out or become endemic depends on cats' epidemiology parameters (e.g., birth rate, horizontal and vertical transmission). However, the underlying assumption that infected cats remain infectious for life and vertically infected cats excrete oocysts is not realistic.

\subsubsection{Cat-environment transmission}

Most models selected studied the transmission of $T$. gondii between susceptible and infected hosts at population level. However, questions like how the transmission of disease among individuals is influenced by the within-host dynamics cannot be answered using those models. The model by Feng et al. (2013) is the first to link between-host and within-host systems for studying the dynamics of $T$. gondii transmission, through their connection to a contaminated environment. However, also according to the authors, their model did not entirely reflect the complexity of the $T$. gondii life-cycle (Feng et al., 2013). In that study, an SI-type of model was used to describe the between-host dynamics of $T$. gondii in cats, and cats were assumed to acquire the infection only by ingesting oocyst contaminated food from the environment (which is not an important route of infection for cats (Dubey et al., 2010)). For the within-host dynamics, three compartments were considered in the system: density of uninfected cells, infected cells and parasite load. This study showed that the $R_{0}$ from the between-host system, within-host 
system and the coupled system determine the stability of the infection-free and the endemic equilibrium points.

Cen et al. (2014) further analyzed the model proposed by Feng et al. (2013), and confirmed that backward bifurcation occurred in the model. Backward bifurcation is the phenomenon in disease transmission models where a stable endemic equilibrium co-exists with a stable disease-free equilibrium when the $R_{0}$ is less than one. The implication of backward bifurcation is that the requirement of $R_{0}$ being less than one is necessary but not always sufficient for disease elimination, making effective control difficult.

\subsubsection{Cat-environment-mouse}

The above models focused on the cat-human transmission route and catenvironment transmission route, but they neglected the intermediate host compartment (e.g., prey animals for felines or livestock/wildlife for human consumption) by either assuming that humans get infected by $T$. gondii through contact with cats or cats get infected through contaminated environment. However, epidemiological studies conducted in Europe have shown that humans mainly become infected via consumption of meat (Bobić et al., 1998; Cook et al., 2000), and cats may get infected from oocysts in the environment but with a much lower probability than from tissue cysts in prey (Dubey, 2010). Three studies concerning the transmission dynamics of $T$. gondii included mice in their models (Jiang et al., 2012; Lélu et al., 2010; Lélu et al., 2013). Lélu et al. (2010) developed a deterministic SIR-type model in which both definitive hosts (cats) and intermediate hosts (mice) were represented in the transmission cycle. In their model, cats become infected through contact with contaminated environment or by ingesting infected prey. They also assumed that cats defecate only in some areas of their habitat. Additionally, decontamination of the environment was considered. They concluded that a threshold of predation rate, depending on the size of the cat population, determines the dynamics of $T$. gondii transmission. When considering a population of 100 cats, if the predation rate is above the threshold of 9 prey/cat/year, the complex life cycle ( $T$. gondii transmitted to a definitive host via predation of infected intermediate hosts) contributed most in spreading the $T$. gondii infection. In contrast, if below the threshold, the spread of infection is predominantly transmitted via a simple life cycle ( $T$. gondii transmitted to definitive hosts via contaminated environment). However, vertical transmission was neglected in both cat and prey population. Although vertical transmission generally only occurs during the acute stage of infection, mice may transmit vertically in subsequent pregnancies (Owen and Trees, 1998).

Based on the same model structure used in 2010 (Lélu et al., 2010), Lélu et al. (2013) further investigated how parasitic manipulation of intermediate host behavior 
(assumed to increase the predation of infected rodents by definitive hosts), vertical transmission and virulence of strains affect $T$. gondii transmission and their effects on the $\mathrm{R}_{0}$. They showed that manipulation is particularly advantageous for virulent strains and in epidemic situations, and the level of manipulation will evolve depending on the sex of the intermediate hosts and the transmission route. In another transmission model including cats, mice and the environment, Jiang et al. (2012) built an agent-based model to explore the transmission process of $T$. gondii in a farm setting and to investigate the impact of oocyst survival and seasonality on cat and rodent seroprevalence. In this model, primary infection and secondary infection of cats were taken into account. The results showed that: (1) most cats are infected through preying on infected mice, while mice are mainly infected through vertical transmission; (2) reducing the number of mice on a farm can lead to eradication of the parasite; (3) intermediate virulent lineages can sustain the disease most efficiently.

\subsection{Intervention}

Of the selected papers, five implemented potential intervention strategies in the transmission model. Mateus-Pinilla et al. (2002) developed the first compartment model for $T$. gondii transmission in a swine farm setting. The model included three compartments: cats, finishing pigs and environment. Subsequently, the effect of vaccinating cats on the prevalence of $T$. gondii in finishing pigs was evaluated. In addition, the values of several model parameters were varied for testing the effects on $T$. gondii prevalence in finishing pigs. They found that a decrease in the initial number of cats on the farm and decreased oocyst survival impacted the prevalence more than vaccinating the cats. Arenas et al. (2010) investigated the transmission of $T$. gondii between cats and the environment (oocysts) under a continuous vaccination schedule for cats. Similar to the results obtained by González-Parra et al. (2009), they also found that the $R_{0}$ completely determines the dynamics of the infection. In contrast to the results by Mateus-Pinilla et al. (2002), Arenas et al. (2010) found that continuous vaccination of cats was more effective than removing the oocysts from the environment according to scenario analysis, and a low vaccination rate (0.1) was enough to reduce the $R_{0}$ to less than one. Based on the model structure from Lélu et al. (2010), Turner et al. (2013) extended the model by taking the virulence of $T$. gondii, vertical transmission, and manipulation of host behavior (predation rate) into account. They investigated the effect of two intervention strategies (cat vaccination program and mouse elimination program) on $T$. gondii transmission and extended the model with an end receiver host, namely sheep. They demonstrated that infection can theoretically be controlled by implementing both control programs in the catmouse-environment system. However, the level of vaccination and/or elimination may not always be achievable. In a setting with 50 cats and 300 mice, the model 
estimated that approximately five years were needed to reduce the number of infected mice to close to zero when implementing the cat vaccination (vaccination rate: 3 week $^{-1}$ ) and the mouse elimination program (elimination rate: 0.8/52 week $\left.{ }^{1}\right)$ together (Turner et al., 2013). They also suggested that it is necessary to control the spread of infection in cats and mice in order to control the spread of infection in the end receivers. By taking the cost of vaccine and infection into account, Sykes (2015) used the transmission model from Arenas et al. (2010) to determine at what cost the cat owners are willing to vaccinate their pets. They found that there is a critical cost threshold above which no one will use the vaccine, whereas a high usage of vaccine could be achieved if the cost is slightly below the threshold. To evaluate the vaccination strategy, they used a payoff value to evaluate the vaccination strategy, which is the benefit an individual derives from implementing the strategy minus the cost of implementing such a strategy. However, the model neglected the infection of cats via prey animals and assumed that the cat owners become infected only via their infected cats. This may lead to an incorrect estimate of the risk of human infection, and subsequently of the cost of infection. Based on the model structure from Lélu et al. (2010), Bonačić Marinović et al. (2019) developed a compartment model and a dose-response relation upon oocyst ingestion to study the effect of cat vaccination in reducing the risk of human $T$. gondii infection. In addition, two modelling approaches regarding how human $T$. gondii infection take places via the contaminated environment were conducted. One approach considered a fixed probability of infection, and the other considered the probability of infection to be proportional to the oocyst abundance. These two approaches can be interpreted as if oocysts are clustered or homogeneously distributed in the environment, respectively. Similar to the finding by Turner et al. (2013), Bonačić Marinović et al. (2019) concluded that a high cat vaccination coverage was needed to reduce the number of human infections, and a high coverage was considered unfeasible for large cat populations.

\subsection{Spatial distribution and outbreak investigation}

Among all included publications, only one studied how landscape structures impact on the spatial distribution of $T$. gondii prevalence in cats and rodents as well as contamination in the environment (Gotteland et al., 2014b). The authors extended the agent-based model developed by Jiang et al. (2012) to describe the transmission dynamics of $T$. gondii and investigated the spatial distribution of $T$. gondii in the environment. In addition, the prevalence of T. gondii in cats and mice, and the soil contamination rate predicted by the model were compared to field data from the same study site for validation purposes. They showed that the relationships between the level of soil contamination and the distance to the nearest farm, and between level of soil contamination and the distance to the 
village center were similar. The model outcomes were also used to produce a risk map of T. gondii contamination in the environment.

One of the selected studies investigated an outbreak of toxoplasmosis among those who visited a riding stable in Atlanta, Georgia, in 1977 (Shonkwiler and Thompson, 1982). The authors used detailed data from individuals who visited the stable (e.g., location, frequency of visits, distance to oocysts and the estimated onset of illness) to predict the epidemic curves and probability of infection.

\section{Discussion}

Mathematical models may be useful tools for studying the transmission of $T$. gondii and evaluating potential intervention strategies, however our knowledge and information on how to develop/apply reliable models and interpret the model results are limited. Our systematic review found that the mathematical models published in the past decades for studying the transmission dynamics and evaluating intervention strategies of $T$. gondii are scarce. Studies of other pathogens found a total number of 388 (Reiner et al., 2013) and 29 (Dixon et al., 2019) mathematical models concerning mosquito-borne pathogen transmission and Taenia solium transmission, respectively. The limited number of $T$. gondii mathematical models may largely be due to the complicated life cycle of the parasite, i.e., multiple hosts and different transmission pathways, which makes it very difficult to model. Thus, models do not always represent reality, for example, the models by Gonzalez-Parra et al. (2009), Ferreira et al. (2017) and Yongzhen et al. (2018) did not include transmission via intermediate hosts (i.e., infection of cats via infected prey, and infection of humans via undercooked or raw meat containing tissue cysts), and have the underlying assumption that vertically infected cats excrete oocysts and infected cats remain infectious for life (i.e., infectious for humans and cats via contact), and the model did not differentiate between the sex of the cats, thus all infected cats were assumed to produce infected offspring at a varying probability. It is questionable whether the conclusions from these models have value in spite of these important omissions compared to current knowledge on $T$. gondii transmission.

Toxoplasmosis causes huge production losses in the small ruminant industry (Stelzer et al., 2019) and has serious public health implications (Weiss and Dubey, 2009). For these reasons it needs to be studied and prevented, and mathematical modelling can help. However, the complicated life cycle of $T$. gondii makes it difficult to choose a modelling approach that is simple but still captures all important aspects. We found that the most common modelling approach for studying $T$. gondii transmission was a deterministic, equation-based compartmental model, but simulation-based agent-based models, a game theory 
model and a common source epidemic stochastic model were also used. Compartment models and agent-based models are two common frameworks for modelling infectious disease. For compartment models, Anderson and May (1992) identified the two most important assumptions: homogeneity and law of mass action. The first one assumes that all individuals in a particular compartment behave in the same manner. The second one means that the rate of change of individuals in a compartment at the next time step is proportional to the number of individuals in the compartment at the current time step. In contrast, agentbased models are a step away from the homogeneity of compartment models, and they are used to simulate autonomous agents and their interactions within a constrained environment over time (Epstein, 2007). Agent-based models have been used for prediction, inference, and the study of hypothetical intervention strategies in the epidemiology field. However, they are often more complicated and require more computation time than compartment models. In the agentbased model developed by Jiang et al. (2012), the complete life cycle of $T$. gondii in a farm setting was considered. Gotteland et al. (2014b) further studied the transmission of $T$. gondii and spatial distribution of oocysts based on the model from Jiang et al. (2013), and obtained results that appear realistic in comparison to data observed in a field study (Afonso et al., 2009; Gotteland et al., 2013; Gotteland et al., 2014b). An advantage of this agent-based model was that the agents in the model (in this case cats and mice) were both autonomous and characterized by their own rules, and it included a detailed description of $T$. gondii transmission. Thus, the structure of this model could be used as a baseline for studying the transmission of T. gondii and evaluating potential control strategies. Moreover, it can be further extended by including other intermediate hosts, like farm animals and humans.

From the selected publications, most modelling work has focused on the epidemiological interaction between hosts and the proliferation dynamics within the hosts separately. To date, more studies have revealed new and interesting insights for disease dynamics by using a linked between-host and within-host model (Gilchrist and Coombs, 2006; Qesmi et al., 2015). A recent systematic review has identified 24 papers across 30 years that include the linked models for different pathogens (Childs et al., 2019). Feng et al. (2013) published the first mathematical model coupling between-host and within-host dynamics of $T$. gondii infection. Cen et al. (2014) further analyzed this coupled model and proved that backward bifurcation can occur in the model. This finding can have important public health implications for controlling T. gondii infection. Although, as the authors acknowledge, the model neglected the prey-predator (rodent-cat) interaction in the transmission process which may have influenced the results. They suggested that the model is more appropriate for an environmental-driven infectious disease with a simpler life cycle than $T$. gondii. 
Our systematic review shows that the two main intervention measures examined in the mathematical models, included cat vaccination and mouse elimination. However, the efficiencies of these intervention measures were difficult to compare, as they were evaluated in models based on different structures and parameter values. For instance, Mateus-Pinilla et al. (2002) indicated that reducing the number of cats on a farm had a greater effect on reducing the prevalence of $T$. gondii than a cat vaccination program, while others (Arenas et al., 2010; Bonačić Marinović et al., 2019; Turner et al., 2013) found that controlling the infection was feasible by vaccinating cats but different vaccination coverages were reported. However, the model by Mateus-Pinilla et al. (2002) did not include the infection of cats via infected prey, thus the role of mice was not evaluated. In addition to the need for tools to better evaluate the efficiency of potential intervention strategies, economic costs should also be considered in future modelling work.

It is known that $T$. gondii is not homogeneously distributed in the environment as oocyst deposition by cats depends on cat density and incidence of $T$. gondii infection (Gotteland et al., 2014a). Oocysts are usually concentrated in defecation sites of cats and the contamination level in environment was associated with season and climatic factors (Schares et al., 2016). The viability of oocysts depends on environmental factors such as humidity, exposure to sunlight and freezing (Dubey et al., 1970; Frenkel and Dubey, 1973). Nonetheless, we could only identify one study describing a model to study the spatial pattern of environmental contamination by $T$. gondii. This type of modelling is mainly hampered by the complexity of integrating the behavior of intermediate and definitive hosts, contamination and infectivity of $T$. gondii oocysts in the environment, and the characteristics of the landscape (Gotteland et al., 2014b). Parameters like detection and survival of oocysts in environmental samples, transmission rate from the environment to cats and rodents, and daily behavior of the hosts are often difficult or unfeasible to quantify. For example, the probability of rodent infection following ingestion of one oocyst was set to one in the model by Gotteland et al. (2014b). However, in reality the viability of oocysts in the environment may be influenced by the duration and conditions of oocyst exposure in the environment. Therefore, more spatial modelling studies to estimate the risk of contamination by T. gondii oocysts would help to get more accurate results when the data gaps are filled.

Only one of the included studies described a model for an outbreak investigation (Shonkwiler and Thompson, 1982). The authors used a discrete-time stochastic model to study an outbreak of toxoplasmosis which occurred among those who visited a riding stable in Atlanta, Georgia, in 1977, and investigated the role of location and frequency of visits in epidemic curve. A few outbreaks of acute toxoplasmosis in humans linked to oocysts have been reported worldwide (Bowie 
et al., 1997; Coutinho et al., 1982; Ekman et al., 2012). However, outbreak investigations are usually hampered by the lack of detailed information.

In conclusion, mathematical models for studying the transmission dynamics of $T$. gondii are scarce considering the high disease burden caused by this parasite. This systematic review provides an overview of the existing mathematical models and summarises the results according to the purposes of the selected studies. The models vary in their structures, assumptions, measured outcomes and investigated intervention strategies. It is clear that a limitation for several of the current mathematical models is the failure to include all relevant transmission routes present in the life cycle of $T$. gondii. Moreover, due to the absence of field data, assumptions often have to be made, which leads to uncertainty of the predicted values and makes it difficult to validate and compare the models. Therefore, currently it is difficult to draw specific conclusions about which prevention and control strategies are the most effective to reduce the burden of toxoplasmosis. Future field work and experiments should include data collection in line with data requirements for these models. In this way, the identified models can be updated and extended to aid the development of effective toxoplasmosis prevention strategies.

\section{Acknowledgements}

This work was a collaboration within the framework of Euro-FBP, the European Network for Foodborne Parasites, COST Action. 


\section{REFERENCES}

Afonso E, Thulliez P, Gilot-Fromont E: Local meteorological conditions, dynamics of seroconversion to Toxoplasma gondii in cats (Felis catus) and oocyst burden in a rural environment. Epidemiol Infect 2009, 138:11051113.

Anderson, R., May, R., 1992. Infectious Diseases of Humans. Oxford University Press.

Arenas, A.J., Gonzalez-Parra, G., Villanueva Mico, R.J., 2010. Modeling toxoplasmosis spread in cat populations under vaccination. Theor. Popul. Biol. 77, 227-237.

Binquet, C., Lejeune, C., Seror, V., Peyron, F., Bertaux, A.-C., Scemama, O., Quantin, C., Béjean, S., Stillwaggon, E., Wallon, M., 2019. The cost-effectiveness of neonatal versus prenatal screening for congenital toxoplasmosis. PLoS One 14, e0221709-e0221709.

Bobić, B., Jevremović, I., Marinković, J., Šibalić, D., Djurković-Djaković, O., 1998. Risk factors for Toxoplasma infection in a reproductive age female population in the area of Belgrade, Yugoslavia. Eur. J. Epidemiol. 14, 605-610.

Bobić, B., Villena, I., Stillwaggon, E., 2019. Prevention and mitigation of congenital toxoplasmosis. Economic costs and benefits in diverse settings. Food Waterborne Parasitol 16, e00058.

Bonačić Marinović, A.A., Opsteegh, M., Deng, H., Suijkerbuijk, A.W.M., van Gils, P.F., van der Giessen, J., 2019. Prospects of toxoplasmosis control by cat vaccination. Epidemics 30, 100380.

Bouwknegt M., Devleesschauwer B., Graham H., Robertson L.J. , van der Giessen J.W.B., the Euro-FBP workshop participants. Prioritisation of food-borne parasites in Europe, 2016. Euro Surveill. 2018;23(9):pii=1700161.

Bowie, W.R., King, A.S., Werker, D.H., Isaac-Renton, J.L., Bell, A., Eng, S.B., Marion, S.A., 1997. Outbreak of toxoplasmosis associated with municipal drinking water. The BC Toxoplasma Investigation Team. Lancet 350, 173-177.

Brauer, F., van den Driessche, P., Wu, J. 2008. Compartmental models in epidemiology, mathematical epidemiology. Springer Berlin Heidelberg, Berlin, Heidelberg, pp. 19-79.

Cen, X., Feng, Z., Zhao, Y., 2014. Emerging disease dynamics in a model coupling within-host and between-host systems. J. Theor. Biol. 361, 141-151.

Childs, L.M., El Moustaid, F., Gajewski, Z., Kadelka, S., Nikin-Beers, R., Smith, J.W., Jr., Walker, M., Johnson, L.R., 2019. Linked within-host and between-host models and data for infectious diseases: a systematic review. PeerJ 7, e7057-e7057.

Cook, A.J., Gilbert, R.E., Buffolano, W., Zufferey, J., Petersen, E., Jenum, P.A., Foulon, W., Semprini, A.E., Dunn, D.T., 2000. Sources of toxoplasma infection in pregnant women: European multicentre case-control study. European Research Network on Congenital Toxoplasmosis. BMJ 321, 142-147.

Coutinho, S.G., Lobo, R., Dutra, G., 1982. Isolation of Toxoplasma from the soil during an outbreak of toxoplasmosis in a rural area in Brazil. J Parasitol. 68, 866-868.

Dietz, K., 1993. The estimation of the basic reproduction number for infectious diseases. Stat. Methods Med. Res. 2, 23-41.

DGS, 2013. Normas e Circulares Normativas. https://www.dgs.pt/directrizes-da-dgs/normas-e-circulares-normativas/norma-n-0372011-de30092011-jpg.aspx. Access date: 30th June 2020.

Dixon, M.A., Braae, U.C., Winskill, P., Walker, M., Devleesschauwer, B., Gabriël, S., Basáñez, M.-G., 2019. Strategies for tackling Taenia solium taeniosis/cysticercosis: A systematic review and comparison of transmission models, including an assessment of the wider Taeniidae family transmission models. PLoS Negl. Trop. Dis. 13, e0007301.

Dubey, J.P., 1995. Duration of immunity to shedding of Toxoplasma gondii oocysts by cats. J Parasitol. 81, 410415.Dubey, J.P., 1998. Advances in the life cycle of Toxoplasma gondii. Int. J Parasitol. 28, 1019-1024.

Dubey, J.P., 2010. Toxoplasmosis of animals and man. CRC Press, Boca Raton.

Dubey, J.P., Miller, N.L., Frenkel, J.K., 1970. The Toxoplasma gondii oocyst from cat feces. J Exp. Med. 132, 636662.

Dunn, D., Wallon, M., Peyron, F., Petersen, E., Peckham, C., Gilbert, R., 1999. Mother-to-child transmission of toxoplasmosis: risk estimates for clinical counselling. Lancet 353, 1829-1833.

ECDC, 2019. Introduction to the Annual Epidemiological Report. In: ECDC. Annual epidemiological report for 2017, Stockholm.

Schares, G., Ziller, M., Herrmann, D.C., Globokar, M.V., Pantchev, N., Conraths, F.J., 2016. Seasonality in the proportions of domestic cats shedding Toxoplasma gondii or Hammondia hammondi oocysts is associated with climatic factors. Int J Parasitol 46, 263-273.

Scientific Opinion on the public health risks associated with food-borne parasites. EFSA Journal 2018;16(12):5495, 
113 pp. https://doi.org/10.2903/j.efsa.

Ekman, C.C., Chiossi, M.F., Meireles, L.R., Andrade Junior, H.F., Figueiredo, W.M., Marciano, M.A., Luna, E.J., 2012. Case-control study of an outbreak of acute toxoplasmosis in an industrial plant in the state of Sao Paulo, Brazil. Rev. Inst. Med. Trop. Sao Paulo 54, 239-244.

Epstein, J.M., 2007. Agent-based computational models and generative social science, generative social science studies in agent-based computational modeling. Princeton University Press.

Ferreira, J.D., Echeverry, L.M., Rincon, C.A.P., 2017. Stability and bifurcation in epidemic models describing the transmission of toxoplasmosis in human and cat populations. Math Methods App Sci 40, 5575-5592.

Feng, Z., Velasco-Hernandez, J., Tapia-Santos, B., 2013. A mathematical model for coupling Within host and between-host dynamics in an environmentally-driven infectious disease. Math. Biosci. 241, 49-55.

Frenkel, J.K., Dubey, J.P., 1973. Effects of freezing on the viability of toxoplasma oocysts. J Parasitol. 59, 587-588.

Gilchrist, M.A., Coombs, D., 2006. Evolution of virulence: interdependence, constraints, and selection using nested models. Theor. Popul. Biol. 69, 145-153.

González-Parra, G.C., Arenas, A.J., Aranda, D.F., Villanueva, R.J., Jódar, L., 2009. Dynamics of a model of Toxoplasmosis disease in human and cat populations. Comput Math App 57, 1692-1700.

Gotteland C., Chaval Y., Villena I., Galan M., Geers R., Aubert D., Poulle M.L., Charbonnel N., Gilot-Fromont E.: Species or local environment, what determines the infection of rodents by Toxoplasma gondii? Parasitology 2013, 141(2):259-268.

Gotteland, C., Gilot-Fromont, E., Aubert, D., Poulle, M.L., Dupuis, E., Dardé, M.L., Forin-Wiart, M.A., Rabilloud, M., Riche, B., Villena, I., 2014a. Spatial distribution of Toxoplasma gondii oocysts in soil in a rural area: Influence of cats and land use. Vet. Parasitol 205, 629-637.

Gotteland, C., McFerrin, B.M., Zhao, X., Gilot-Fromont, E., Lélu, M., 2014b. Agricultural landscape and spatial distribution of Toxoplasma gondii in rural environment: an agent-based model. Int. J Health Geogr. 13, 45.

Jiang, W., Sullivan, A.M., Su, C., Zhao, X., 2012. An agent-based model for the transmission dynamics of Toxoplasma gondii. J Theor. Biol. 293, 15-26.

Jones, J.L., Parise, M.E., Fiore, A.E., 2014. Neglected parasitic infections in the United States: toxoplasmosis. Am J Trop Med Hyg 90, 794-799.

Lélu, M., Langlais, M., Poulle, M.L., Gilot-Fromont, E., 2010. Transmission dynamics of Toxoplasma gondii along an urban-rural gradient. Theor. Popu. Biol. 78, 139-147.

Lélu, M., Langlais, M., Poulle, M.L., Gilot-Fromont, E., Gandon, S., 2013. When should a trophically and vertically transmitted parasite manipulate its intermediate host? The case of Toxoplasma gondii. P Roy Soc BBiol Sci 280, 20131143.

Mateus-Pinilla, N.E., Hannon, B., Weigel, R.M., 2002. A computer simulation of the prevention of the transmission of Toxoplasma gondii on swine farms using a feline T. gondii vaccine. Prev. Vet. Med. 55, $17-36$.

Miller, N.L., Frenkel, J.K., Dubey, J.P., 1972. Oral infections with Toxoplasma cysts and oocysts in felines, other mammals, and in birds. J Parasitol. 58, 928-937.

Opsteegh, M., Kortbeek, T.M., Havelaar, A.H., van der Giessen, J.W., 2015. Intervention strategies to reduce human Toxoplasma gondii disease burden. Clin. Infect. Dis. 60, 101-107.

Peyron, F., Mc Leod, R., Ajzenberg, D., Contopoulos-loannidis, D., Kieffer, F., Mandelbrot, L., Sibley, L.D., Pelloux, H., Villena, I., Wallon, M., Montoya, J.G., 2017. Congenital Toxoplasmosis in France and the United States: one parasite, two diverging approaches. PLoS Negl. Trop. Dis. 11, e0005222-e0005222.

Owen M.R., Trees A.J. Vertical transmission of Toxoplasma gondii from chronically infected house (Mus musculus) and field (Apodemus sylvaticus) mice determined by polymerase chain reaction. Parasitology. 1998;116 ( Pt 4):299-304. doi:10.1017/s003118209700231x

Qesmi, R., Heffernan, J.M., Wu, J., 2015. An immuno-epidemiological model with threshold delay: a study of the effects of multiple exposures to a pathogen. J Math. Biol. 70 343-366. 
Reiner, R.C., Jr., Perkins, T.A., Barker, C.M., Niu, T., Chaves, L.F., Ellis, A.M., George, D.B., Le Menach, A., Pulliam, J.R., Bisanzio, D., Buckee, C., Chiyaka, C., Cummings, D.A., Garcia, A.J., Gatton, M.L., Gething, P.W., Hartley, D.M., Johnston, G., Klein, E.Y., Michael, E., Lindsay, S.W., Lloyd, A.L., Pigott, D.M., Reisen, W.K., Ruktanonchai, N., Singh, B.K., Tatem, A.J., Kitron, U., Hay, S.I., Scott, T.W., Smith, D.L., 2013. A systematic review of mathematical models of mosquito-borne pathogen transmission: 1970-2010. J R Soc. Interface 10, 20120921.

Robert-Gangneux, F., Darde, M.L., 2012. Epidemiology of and diagnostic strategies for toxoplasmosis. Clin. Microbiol. Rev. 25, 264-296.

Rougier S, Montoya JG, Peyron F. Lifelong persistence of toxoplasma cysts: A questionable dogma? Trends Parasitol. 2017;33 2:93-101; doi: 10.1016/j.pt.2016.10.007.

Shonkwiler, R., Thompson, M., 1982. Common source epidemics II: Toxoplasmosis in Atlanta. Bull. Math. Biol. 44, 377-398.

Stelzer, S., Basso, W., Benavides Silván, J., Ortega-Mora, L.M., Maksimov, P., Gethmann, J., Conraths, F.J., Schares, G., 2019. Toxoplasma gondii infection and toxoplasmosis in farm animals: Risk factors and economic impact. Food Waterborne Parasitol. 15, e00037.

Stillwaggon, E., Carrier, C.S., Sautter, M., McLeod, R., 2011. Maternal serologic screening to prevent congenital toxoplasmosis: a decision-analytic economic model. PLoS Negl Trop Dis 5, e1333.

Sykes, D.R., J., 2015. A game-theoretic approach to valuating toxoplasmosis vaccination strategies. Theor. Popul. Biol. 105, 33-38.

Tenter, A.M., Heckeroth, A.R., Weiss, L.M., 2000. Toxoplasma gondii: from animals to humans. Int. J Parasitol. 30, 1217-1258.

Torgerson, P.R., Mastroiacovo, P., 2013. The global burden of congenital toxoplasmosis: a systematic review. Bull World Health Organ 91, 501-508.

Turner, M., Lenhart, S., Rosenthal, B., Zhao, X., 2013. Modeling effective transmission pathways and control of the world's most successful parasite. Theor. Popul. Biol. 86, 50-61.

Weiss, L.M., Dubey, J.P., 2009. Toxoplasmosis: A history of clinical observations. Int. J Parasitol. 39, 895-901.

Yongzhen, P., Xuehui, J., Changguo, L., Shujing, G., 2018. Dynamics of a model of Toxoplasmosis disease in cat and human with varying size populations. Math Comput Simulat 144, 52-59. 


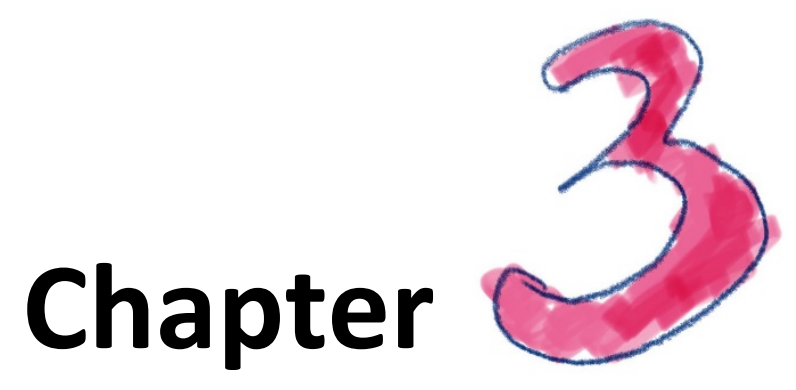

\section{Risk factors related to Toxoplasma gondii seroprevalence in indoor-housed Dutch dairy goats}

Preventive Veterinary Medicine, 2016, 124, 45-51

Huifang Deng ${ }^{1,2}$, Cecile Dam-Deisz ${ }^{1}$, Saskia Luttikholt ${ }^{3}$, Miriam Maas ${ }^{1}$, Mirjam Nielen ${ }^{2}$, Arno Swart ${ }^{1}$, Piet Vellema ${ }^{3}$, Joke van der Giessen ${ }^{1}$, Marieke Opsteegh ${ }^{1}$

Authors' affiliations:

${ }^{1}$ Centre for Infectious Disease Control - Zoonoses and Environmental Microbiology, National Institute for Public Health and the Environment, Bilthoven, the Netherlands

${ }^{2}$ Faculty of Veterinary Medicine, Utrecht University, Utrecht, the Netherlands

${ }^{3}$ Department of Small Ruminant Health, GD Animal Health, Deventer, the Netherlands 


\section{Abstract}

Background: Toxoplasma gondii can cause disease in goats, but also has impact on human health through food-borne transmission. Our aims were to determine the seroprevalence of $T$. gondii infection in indoor-housed Dutch dairy goats and to identify the risk factors related to $T$. gondii seroprevalence.

Methods: Fifty-two out of ninety approached farmers with indoor-kept goats (58\%) participated by answering a standardized questionnaire and contributing 32 goat blood samples each. Serum samples were tested for $T$. gondii SAG1 antibodies by ELISA and results showed that the frequency distribution of the $\log _{10}$-transformed OD-values fitted well with a binary mixture of a shifted gamma and a shifted reflected gamma distribution.

Results: The overall animal seroprevalence was $13.3 \%$ (95\% Cl: $11.7 \%-14.9 \%)$, and at least one seropositive animal was found on $61.5 \%$ (95\% Cl: $48.3 \%-74.7 \%)$ of the farms. To evaluate potential risk factors on herd level, three modeling strategies (Poisson, negative binomial and zero-inflated) were compared. The negative binomial model fitted the data best with the number of cats (1-4 cats: IR: $2.6,95 \%$ Cl: $1.1-6.5 ;>=5$ cats: IR: $14.2,95 \% \mathrm{Cl}: 3.9-51.1)$ and mean animal age (IR: $1.5,95 \%$ $\mathrm{Cl}: 1.1-2.1)$ related to herd positivity. In conclusion, the ELISA test was $100 \%$ sensitive and specific based on binary mixture analysis.

Conclusions: T. gondii infection is prevalent in indoor housed Dutch dairy goats but at a lower overall animal level seroprevalence than outdoor farmed goats in other European countries, and cat exposure is an important risk factor. 


\section{Introduction}

Toxoplasma gondii ( $T$. gondii) is a zoonotic protozoan parasite that may cause serious disease in humans, especially when primary infection is acquired during pregnancy. In the Netherlands, disease burden of fourteen food-borne pathogens was estimated using disability adjusted life years (DALY) as a summary measure of public health, the results showed that $T$. gondii had the highest disease burden at both population and individual level (Havelaar et al., 2012).

In goats, toxoplasmosis causes abortion and stillbirth, and is thus a source of economic loss to goat farmers (Moraes et al., 2011; van Engelen et al., 2014). Moreover, goats are considered important sources of human infection, especially for ethnic groups that commonly consume goat products (Tenter et al., 2000). Tissue cysts of $T$. gondii are responsible for infections via meat (Jones and Dubey, 2012), and T. gondii tachyzoites can be excreted in goat's milk and can survive the raw fresh cheese-making process (Dubey et al., 2014). Infections due to the consumption of raw goat's milk have been reported (Sacks et al., 1982). There is a positive relationship between detection of antibodies against $T$. gondii in goats and presence of tissue cysts in their meat (Dubey et al., 2011). This correlation was also found between seropositivity and presence of $T$. gondii DNA in goat milk (Spišák et al., 2010). Therefore, the prevalence of antibodies in goats can be used to identify risk factors for infection but also gives an indication of the risk of infection for consumers through consuming raw goat products.

The reported percentage of $T$. gondii seropositive goats varied greatly among countries. In Europe, the seroprevalence of T. gondii in goats was estimated at $17 \%$ in Norway (Stormoen et al., 2012), 18.5\% in north Portugal (Lopes et al., 2013), $25.1 \%$ in southern Spain (Garcia-Bocanegra et al., 2013), 30.7\% in Greece (Tzanidakis et al., 2012), 52.8\% in Romania (lovu et al., 2012), and 60.6\% in Italy (Mancianti et al., 2013). Regional variation of seroprevalence may be caused by differences in study population, study year and climate as well as differences in serological tests and criteria of cut-off value used in the test (Tenter et al., 2000). Reported risk factors associated with $T$. gondii infection in goats are age, presence of cats, management system (i.e. extensive/intensive), source of drinking water and abortion history (Cavalcante et al., 2008; Tzanidakis et al., 2012; Gebremedhin et al., 2013; van Engelen et al., 2014).

In the Netherlands, several serological studies concerning $T$. gondii have been conducted, mainly in swine, poultry, cattle and sheep. The results showed a high seroprevalence in sheep (Opsteegh et al., 2010) compared to poultry and swine (van Knapen et al., 1982; van der Giessen et al., 2007). However, little is known concerning the seroprevalence of $T$. gondii in dairy goats. In 1998, epidemiological 
data on $T$. gondii infection in goats in the Netherlands were collected from ten Dutch goat farms, including three farms with a $T$. gondii abortion history. The mean seroprevalence was $47 \%$ (ranging from $5 \%$ to $90 \%$ ) (Antonis et al., 1998). Since then, the number of dairy goats has nearly tripled, whereas the number of farms has decreased by $40 \%$ (Statistics Netherlands, 2015). This intensification of farming is associated with changes in farm management such as year round indoor-housing of dairy goats. In pigs and poultry, a lower seroprevalence of $T$. gondii infection was found in indoor farming systems than in outdoor farming systems (van der Giessen et al., 2007; Maksimov et al., 2011). Therefore, indoor-housed dairy goats are expected to have a low seroprevalence of $T$. gondii.

The objectives of this study were to determine the seroprevalence and to identify risk factors associated with $T$. gondii seroprevalence in Dutch indoor-housed dairy goats. A commercial indirect ELISA was used to test 1664 goat sera and serological results were analyzed using binary mixture models. Potential risk factors were evaluated by comparing Poisson, negative binomial and zero-inflated regression models.

\section{Material and methods}

\subsection{Study population}

In 2013, 451,377 goats were present in the Netherlands according to the Identification and Registration (I\&R) database. Those animals were kept on 10,783 small goat farms ( $\leq 31$ goats) and 546 professional goat farms ( $\geq 32$ goats), of which 349 large dairy goat farms ( $\geq 50$ goats). Most of the dairy goat farms use a deep litter housing system with dry straw as bedding material (Schimmer et al., 2011). This study focused on indoor-housed Dutch dairy goats on commercial farms with more than 100 goats that participated in an accreditation program for caprine arthritis encephalitis (CAE) or caseous lymphadenitis (CL), as carried out by GD Animal Health.

\subsection{Data collection and sample size}

A standardized questionnaire was designed to measure the exposure to putative risk factors. The questionnaire (available on request) included 12 closed-ended questions on: presence of (young) cats, number of cats on farm, problems with mice/rats, water sources, use of automated mixer-feeder, storage of silage, history of outdoor access, replacement policy, and presence of other farm animals. Information on farm size and animal ages were collected from GD Animal Health database. To avoid effects from destocking and restocking during the Dutch $Q$ fever epidemic (2009-2010), all farm management questions were referring to the past two years. During the data collection period (August 2013 to June 2014), 90 farms 
submitted goats' venous blood samples to GD Animal Health as part of the accreditation programs. These 90 farmers were asked to participate in this study, to which 52 agreed. The questionnaire and informed consent form were sent by email and regular mail to the 52 Dutch dairy goat farms and completed by the farm owner or manager in July-August 2014. On each farm, blood samples had been collected by the veterinarian from 44 to149 animals of more than one year old, according to the CAE and CL monitoring sampling schemes provided by GD Animal Health. To obtain an equally precise estimate of on-farm T. gondii prevalence for all farms, it was decided to test the same number of animals per farm. With 52 participating farms, available ELISA tests were sufficient for 32 goats per farm, thus, 32 frozen serum samples per farm were selected and tested at National Institute for Public Health and the Environment.

The maximum possible within-herd prevalence detectable with 32 sampled goats and at a confidence level of $95 \%$ was estimated using winepi.net. The minimum detectable incidence rate ratio (IR) between exposure to risk factors and infection (presence of toxoplasmosis) at herd level in this study with a sample size of 52 farms was determined based on: (1) the relative frequency of exposure among non-infected farms: $50 \%,(2)$ the ratio between non-infected and infected farms: 3 , (3) a confidence level of $95 \%$ and allowable error of $10 \%$ (Noordhuizen et al., 2001).

\subsection{Serological assay}

Individual goat serum samples were tested with a commercial indirect enzymelinked immune sorbent assay (ELISA) (ID Screen Toxoplasmosis Indirect Multispecies; ID.VET Innovative Diagnostics, France) to determine the presence of $T$. gondii specific P30 (SAG1) antibodies. All steps were carried out according to the instruction of the manufacturer. Every serum sample was tested in duplicate; plate-to-plate variation of the optical density values (OD-value) was corrected by using linear regression on the control sera tested on every plate (Opsteegh et al., 2010). A plate was retested if replicates of one control had a coefficient of variation (CV) above 20\% (Reed et al., 2002), or if the mean OD-value of the positive control was lower than 0.350 and/or the ratio of the mean OD-value of the positive and negative controls was lower than 3 . Individual sera were retested if the replicates showed different statuses according to the cut-off values provided by the manufacturer. Information from the farm questionnaires was blinded to the laboratory technician. 


\subsection{Data analysis}

Information collected in the questionnaires and the serological tests results were coded and entered in Microsoft Access and Microsoft Excel, and statistical analysis was performed using SPSS software version 20 and R 3.03 (IBM Corp, 2011; R Core Team, 2014).

\subsubsection{Binary mixture analysis and estimation of seroprevalence}

A suitable cut-off with corresponding estimates of diagnostic performance was obtained by fitting binary mixture models to the $\log _{10}$-transformed OD-values from the ELISA (Jacobson, 1998; Opsteegh et al., 2010). Visual inspection of the histogram of the data revealed flattened tails, thus suggesting that the components of the mixture may not be normally distributed. Therefore, pragmatically, all combinations of (1) normal and (2) shifted (along the x-axis) and optionally reflected (along the $x$-axis, when used for the positive component) gamma distributions were fitted and compared based on Akaike information criterion (AIC) values. Normal distributions and mixing parameter (prevalence) were fitted as described before (Opsteegh et al., 2010). For (reflected) gamma distributions, shape $(\alpha)$ and rate $(\beta)$ parameters and a shift along the $x$-axis were estimated for both the negative and positive components. A cut-off value was determined at the maximum sum of sensitivity and specificity based on the final binary mixture model. Overall animal level seroprevalence was calculated based on the fraction of animals with an ELISA OD-value above the cut-off value provided by the manufacturer, and compared to the seroprevalence obtained from the binary mixture model. A farm was considered positive if at least one goat on the farm was positive in ELISA. Ninety-five percent confidence intervals for seroprevalence were based on normal approximation for the binomial distribution.

\subsubsection{Risk factor analysis}

Frequency tables of categorical variables and frequency distributions of continuous variables were constructed. Risk factors were analyzed on farm level, with mean age of the 32 selected animals per farm included as sample age. Three different modeling strategies for count data were compared: Poisson, negative binomial (NB) and zero-inflated models. Potential risk factors on farm level were analyzed by both Poisson and NB models with number of seropositive animals per farm as dependent variable and the 32 tested animals per herd as offset. In the first step, bivariable associations between each potential risk factor and the dependent variable were estimated. After testing the effect of age on seropositivity on individual animal level using a binary logistic regression model with a random herd effect, sample age was always included in the farm level models to adjust the estimates. All independent variables with $p$-value $\leq 0.2$ were selected and the associations between those variables were tested with Chi-square test before 
being entered into the multivariable regression model in the second step. If variables were found to be correlated, decisions on inclusion of variables were made based on biological relevance, completeness of data, and the strength of relationship between the outcome and the putative risk factors. Potential confounding was determined by the change $(\geq 20 \%)$ of estimates for other independent variables before and after the factor entering the model (Dohoo et al., 2009). Missing values were excluded in the bivariable analysis, but they were coded as additional categories in the multivariable step. The model was built in a backward elimination process and two-way interaction terms of the biologically plausible variables in the multivariable model were tested. All variables with a $p$ value less than 0.05 in the likelihood ratio test were kept in the model. Risk was expressed as an incidence rate ratio (IR) with 95\% confidence interval (Dohoo et al., 2009). The fit of the models were checked using the Pearson goodness-of-fit test and dispersion parameter. A choice was made between the Poisson model and the NB model based on the likelihood ratio test.

Finally, because of the potential presence of excess zeros in the data, a zero-inflated model was built. First, the associations between all the variables were tested with Chi-square test. All variables except correlated ones were entered into both the count and zero parts of the zero-inflated model. Next, this full model was reduced by automatic model selection based on finite sample corrected AIC (AICC). The process was performed using MuMIn package in R (Barton', 2014). The fit of the Poisson or NB model and the respective zero-inflated model was compared using a Vuong test (Vuong, 1989).

\section{Results}

\subsection{Descriptive statistics}

Epidemiological data were collected from 52 out of 90 approached farms (58\%) and a total number of 1664 serum samples (32 per farm) were obtained from those farms. The participating farms originated from all provinces in the Netherlands, except Groningen and Zeeland. The mean number of goats older than one year on those 52 farms was 792 (ranging from 162 to 2083) (Fig. 1), and was not different between positive (784) and negative farms (805) (independent samples t-test, $p<0.05)$. For $93 \%$ of the 1664 sampled animals, age information was available. Mean age of all individual animals was 3.3 years, and varied between 1 and 11.7 years (Fig. 2). The mean age per farm, which was used in further analyses as sample age, ranged from 1.3 to 7.2 years. 


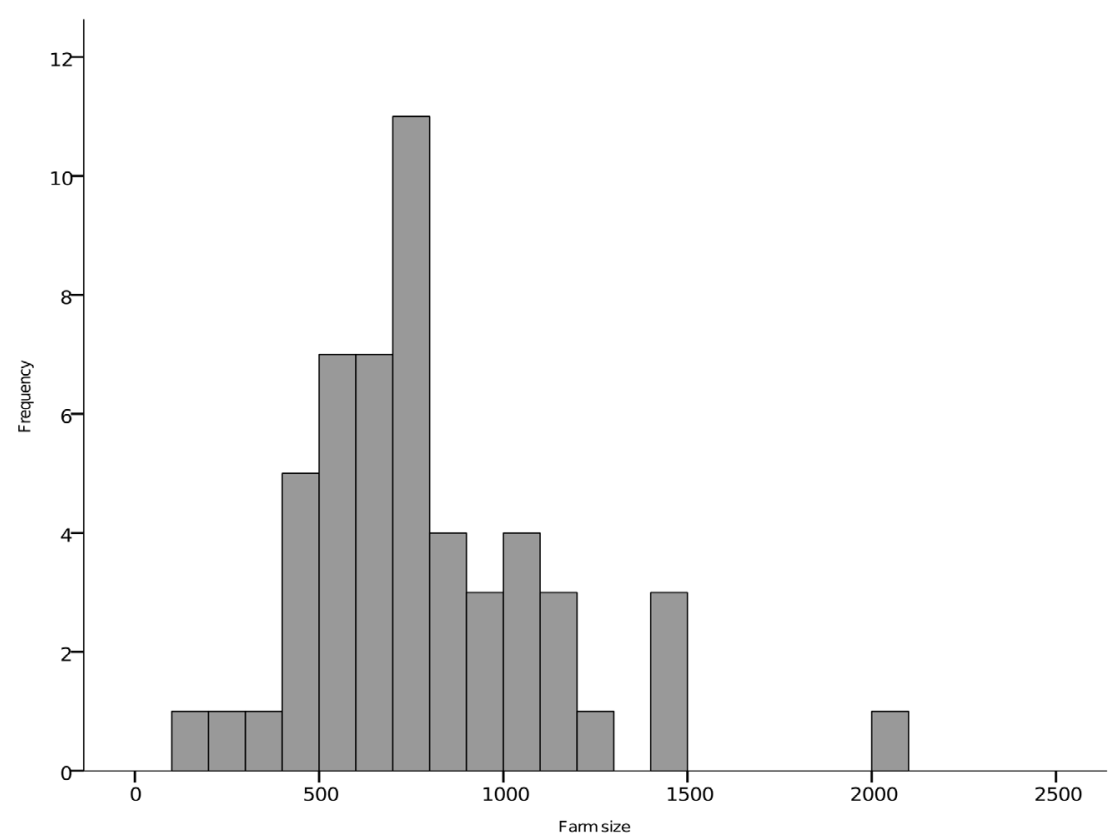

Figure 1. Frequency distribution of farm size for 52 indoor Dutch dairy goat farms participating in $T$. gondii risk factor study.

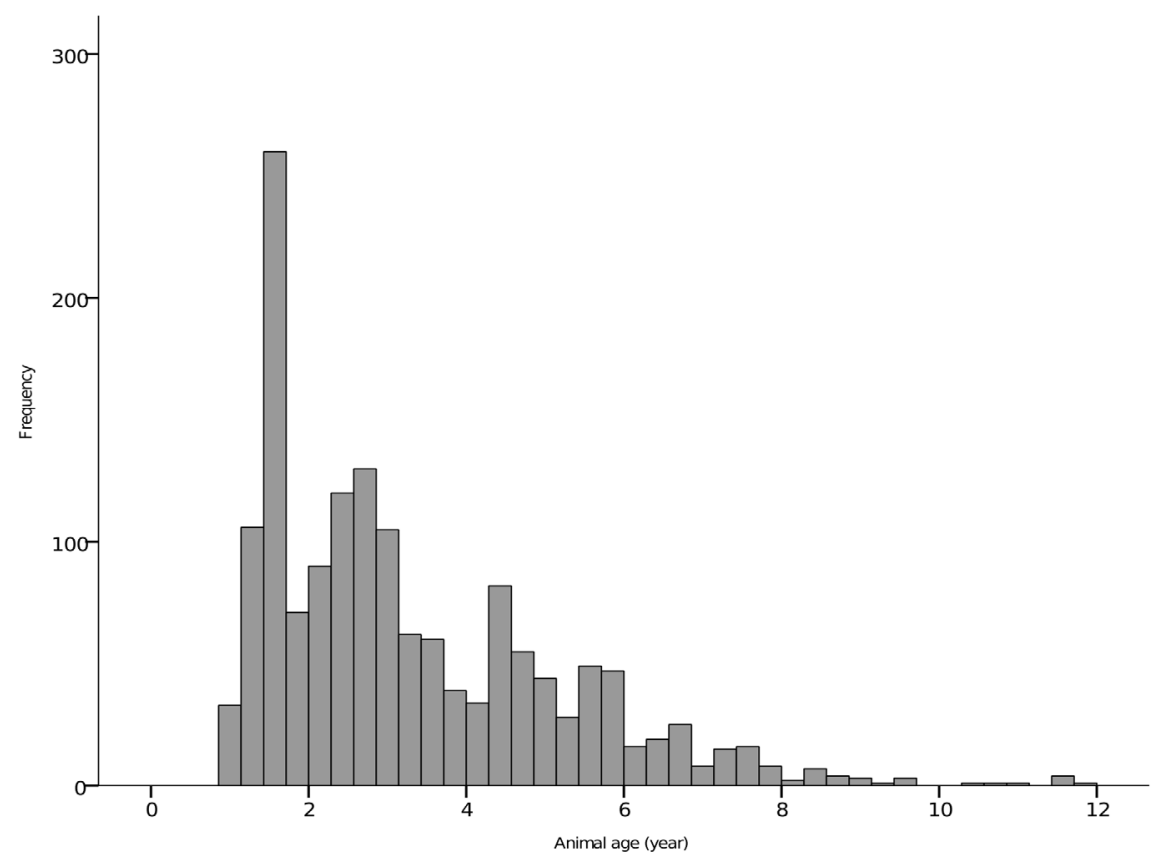

Figure 2. Frequency distribution of age (in years) for 1664 dairy goats from 52 indoor farms participating in T. gondii risk factor study in the Netherlands. 


\subsection{Serological results}

Based on the cut-off values from the commercial test, 221 out of 1664 (13.3\%, 95\% $\mathrm{Cl}: 11.7 \%-14.9 \%$ ) dairy goats scored positive (S/P\% $\geq 50 \%$, which corresponded to a corrected OD-value of 0.93 ) and 1443 scored negative (S/P\% $\leq 40 \%$, which corresponded to a corrected OD-value of 0.76). Samples between 40 and 50\% S/P\% were defined as doubtful. On initial testing, for three animals, the duplicates showed a positive and a doubtful result. After retesting, all of the duplicates were consistently positive. Out of the 52 farms, 32 (61.5\%, 95\% Cl: 48.3\%-74.7\%) had one or more seropositive dairy goat(s) present and were defined as positive farms. Among the positive farms, the seroprevalence of $T$. gondii varied from $3.1 \%$ to $96.9 \%$ (Fig. 3). The odds ratio for seropositivity of $T$. gondii increased significantly with individual age (OR: $1.4,95 \% \mathrm{Cl}: 1.2-1.6)$ based on a binary logistic regression model with animal status as outcome variable and a random herd effect $(p<0.001)$ (details not shown).

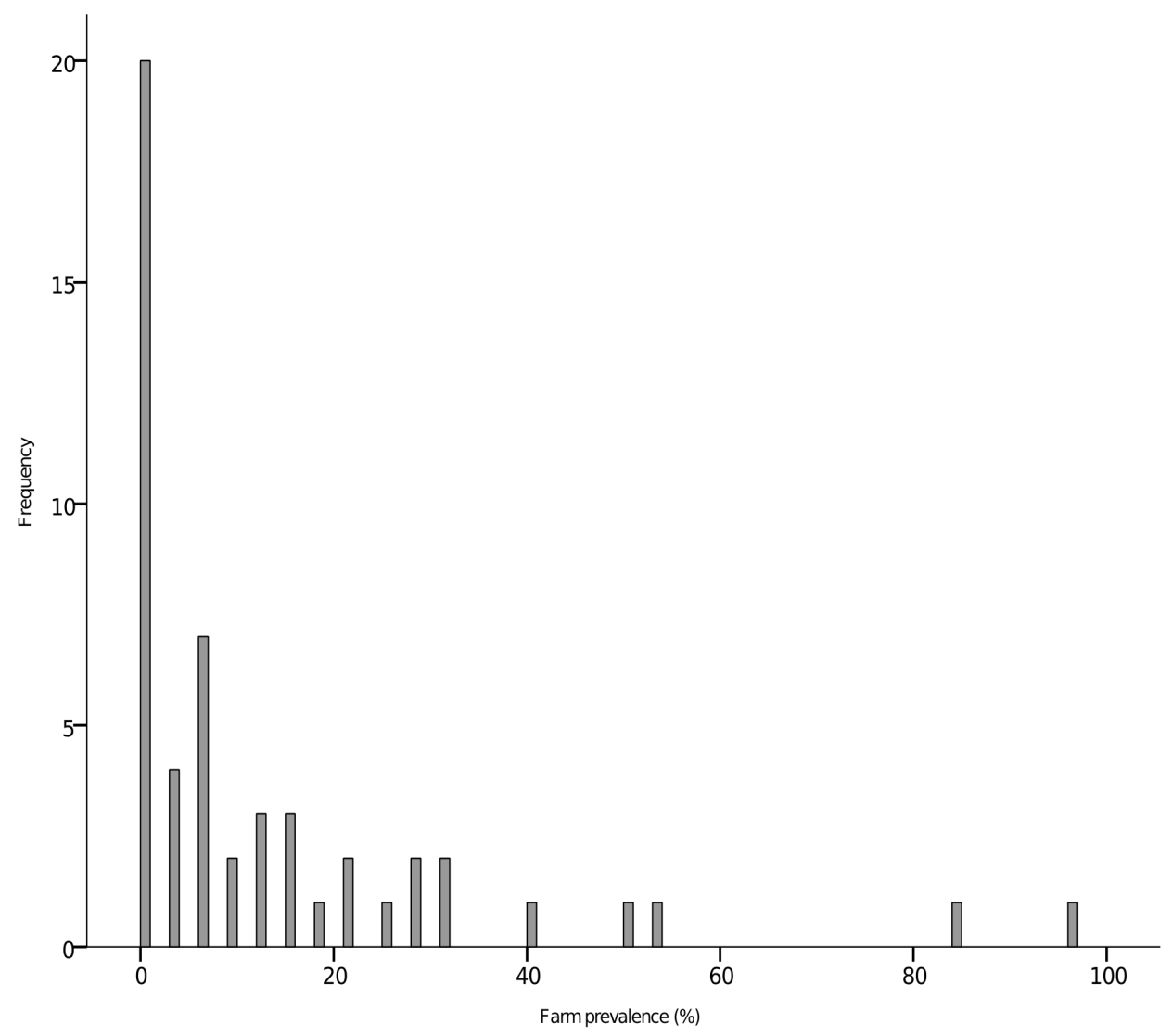

Figure 3. Frequency distribution of within farm seroprevalence of $T$. gondii in Dutch dairy goats at indoor farms ( $n=52$ farms, with 32 animals tested per farm). 
The frequency distribution of the $\log _{10}$-transformed corrected OD values from the ELISA test clearly showed two separated distributions. The binary model with a mixture of a shifted gamma and a shifted reflected gamma distribution had the lowest AIC value and fitted data best (Fig. 4). The negative component is described by Gamma ( $\alpha=8.2, \beta=24.4$ ) shifted by 0.8 along the $x$-axis, and the positive component is described by a reflected Gamma ( $\alpha=85.2, \beta=10.3$ ) shifted by 1.5 along the $x$-axis. The overall seroprevalence was estimated at $13.2 \%$ based on this binary model. The optimum cut-off OD-value was 1.05 (the vertical line in Fig. 4), with both sensitivity and specificity estimated at $100.0 \%$. As there were no goats with OD-values between 0.76 and 1.05, the choice of the cut-off value (manufacturer or binary mixture model) did not affect the scoring of the goats or the estimates of seroprevalence.

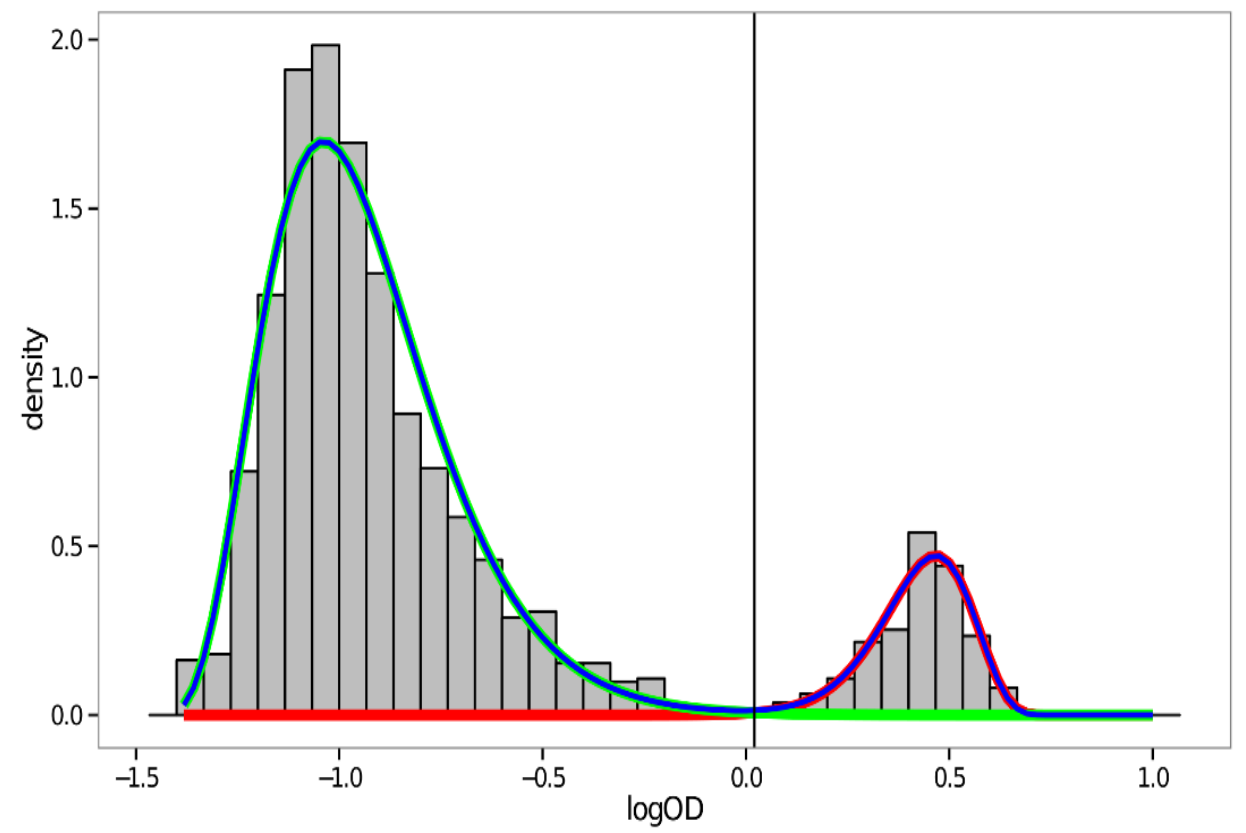

Figure 4. Frequency distribution of log10-transformed OD values from 1664 Dutch dairy goats at indoor farms in T. gondii ELISA (bars), fitted with a mixture of a shifted gamma (left) and a shifted reflected gamma distribution (right), and cut-off value $(\log O D=0.02)$ (vertical line).

\subsection{Risk factor analysis on herd level}

The final multivariable Poisson regression model showed lack of fit (Pearson Chisquare test, $p<0.05$ ) and over-dispersion (Deviance/degree of freedom $=4.2$, where $>1$ indicates over-dispersion). The likelihood ratio test suggested that the 
negative binomial (NB) model fitted significantly better than the Poisson model $(p<0.001)$. In addition, a zero-inflated NB model was built, but the Vuong test indicated superior fit of the NB model $(p<0.001)$. Six variables with a $p<0.2$ were found in the bivariable NB regression analysis (Table 1). However, number of cats was significantly correlated with presence of cats, access of cats to the stable and presence of young cats (Pearson Chi-Square, $p<0.05$ ), therefore only number of cats, use of mixer-feeder, presence of other farm animals and sample age were entered into the multivariable NB regression model. The final model maintained only two predictors: number of cats (1-4 cat: IR: $2.6,95 \% \mathrm{Cl}: 1.1-6.5$; >= 5 cats: IR: 14.2, 95\% Cl: 3.9-51.1) and sample age (IR: 1.5, 95\% Cl: 1.1-2.1) (Table 2). The NB model fitted the data well (Pearson Chi-square test, $p<0.05$ ) with a dispersion coefficient of 1.4 (95\% Cl: 0.8-2.5).

\section{Discussion}

A commercial ELISA test (ID Screen Toxoplasmosis Indirect Multi-species; ID.VET Innovative Diagnostics, France) was used to determine the presence of $T$. gondii specific P30 (SAG1) antibodies in goat serum samples. This is a multispecies ELISA, with a sensitivity and specificity of $86 \%$ and $99 \%$ respectively in experimentally infected swine (Bokken et al., 2012), and a sensitivity between $95 \%$ and $97 \%$ and a specificity of $97 \%$ in Romanian household cats (Györke et al., 2011). No information on sensitivity and specificity of the assay for use with goat sera was available. Therefore, the assay was evaluated by binary mixture analysis on the frequency distribution of observed $\log _{10}$-transformed OD-values. The histogram showed two clearly separated components with an apparent right-skew for the seronegative component and left-skew for the seropositive component. When fitted with a binary mixture model, a cut-off value with sensitivity and specificity both at $100 \%$ could be found. This indicates that the serological assay has a strong discriminatory power for classifying dairy goats as positive or negative for $T$. gondii antibodies. However, external validation based on the results with a different assay would be valuable. The skewness of the distributions is confirmed by the superior fit of the combination of a shifted gamma and a shifted reflected gamma distribution and may have resulted from OD measurements outside the linear relation between OD and antibody concentration. Overall animal level seroprevalence of $T$. gondii infection was estimated at $13.3 \%$ (95\% Cl: $11.7 \%-14.9 \%)$. This seroprevalence is much lower than previously reported in goats (47\%) and adult sheep $(48.1 \%)$ in the Netherlands (Antonis et al., 1998; Opsteegh et al., 2010), and also low compared to results in goats in many other countries, ranging from $30.7 \%$ up to $77 \%$ (Tenter et al., 2000; lovu et al., 2012; Tzanidakis et al., 2012; Mancianti et al., 2013). 


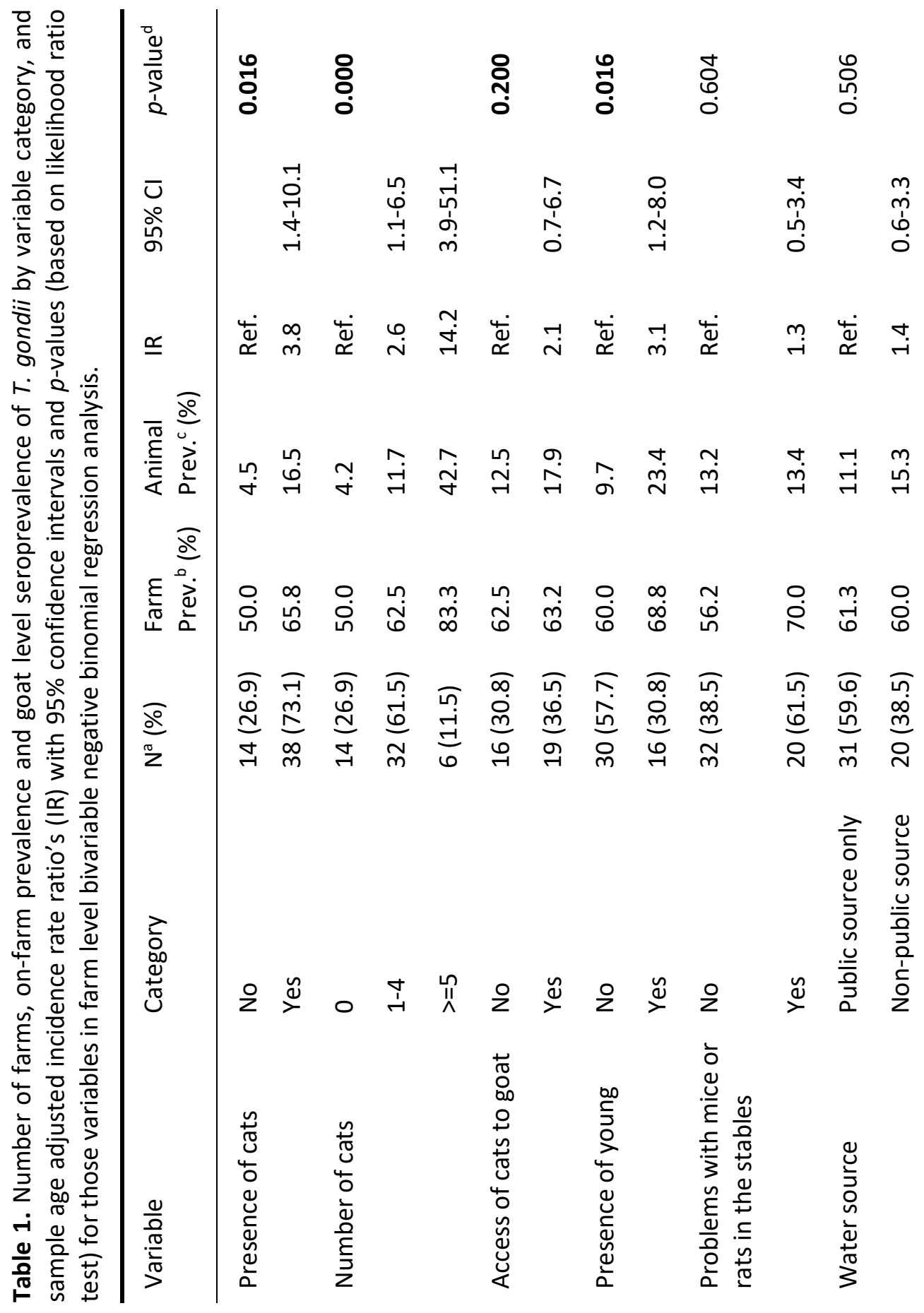




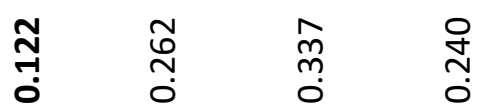

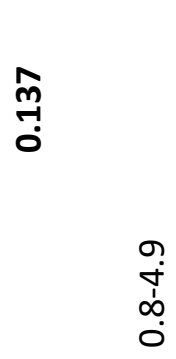

败

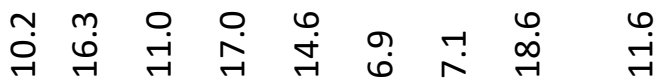

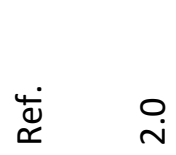

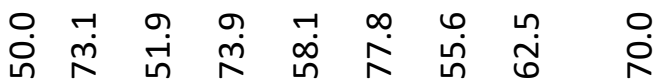

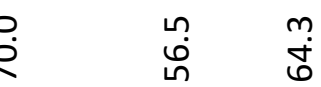

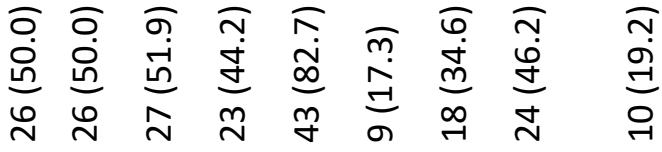

$\begin{array}{ll}\underset{N}{D} & \stackrel{\infty}{n} \\ \underset{n}{n} & \stackrel{\infty}{N}\end{array}$

$\frac{7}{c}$
$\frac{1}{0}$
$\frac{0}{\pi}$
อิ

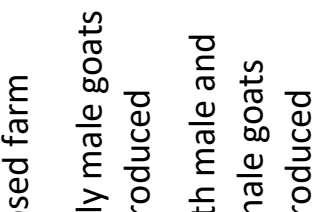

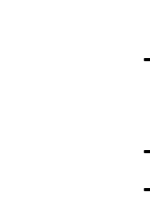

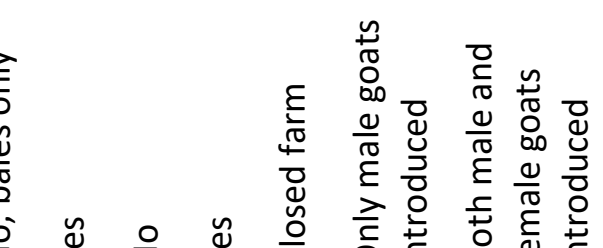

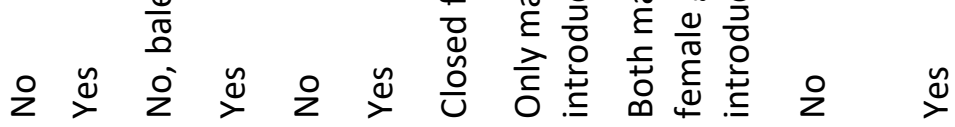

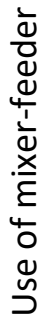

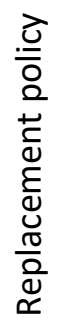

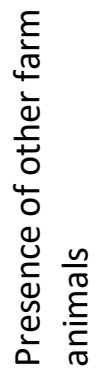

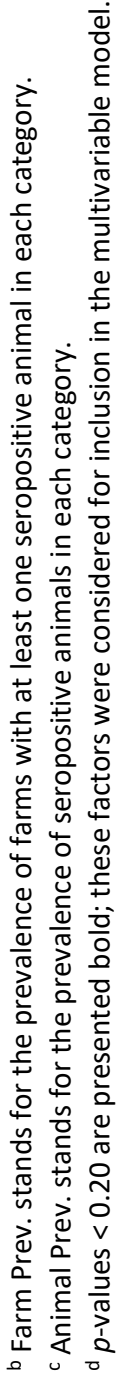


Table 2. Incidence rate ratios (IR) for variables associated $(p<0.05$ in likelihood ratio test) with Toxoplasma gondii seropositivity at dairy goat farms in multivariable negative binomial regression analysis.

\begin{tabular}{llllll}
\hline Variable & Category & $\mathrm{N}(\%)$ & $\mathrm{IR}$ & $95 \% \mathrm{Cl}$ & $p$-value \\
\hline Number of cats & 0 & $14(26.9)$ & Ref. & & 0.000 \\
& $1-4$ & $32(61.5)$ & 2.6 & $1.1-6.5$ & \\
Sample age & Continuous & NA & 1.5 & $1.1-2.1$ & 0.011 \\
\hline
\end{tabular}

Another interesting finding was that on $38 \%$ of the 52 farms all tested goats were seronegative, whereas seropositive animals were present on all ten investigated farms (including three with a T. gondii abortion history) in 1998 (Antonis et al., 1998). Selection bias is expected to be limited in our study, as the majority of large dairy goat farms in the Netherlands participate in the CAE and CL monitoring programs from GD Animal Health, and the 90 farms were approached because they were planned to submit goat blood samples to the institute during the study period. However, it cannot be ruled out that the farmer's willingness to participate is influenced by, for example, a known $T$. gondii abortion history at the farm. In conclusion, indoor housing thus appears to reduce exposure of goats to $T$. gondii but not as much as has been shown for pigs and poultry (van der Giessen et al., 2007). This was expected as goat housing is less confined than pig or poultry housing, with e.g., natural ventilation and introduction of bedding, silage and roughage into the stable.

A short standardized questionnaire was used for farm level risk factors. Potential risk factors without anticipated variation between Dutch herds, i.e. the bedding material, type of housing and feeding system, were excluded from the questionnaire. To evaluate the potential risk factors, three modeling strategies were compared. The dataset was clustered and the outcome variable showed overdispersion (Fig. 3). Therefore, a NB model was built, and the dispersion coefficient in the NB model was significantly larger than zero, indicating this model was more appropriate than a Poisson model. To evaluate the presence of excess zeros, a zeroinflated NB model was additionally built, but the Vuong test indicated that the NB model fitted better. Number of cats remained a significant predictor in the NB model for the number of seropositive goats in the farm, with an increased positive association for farms with 1-4 cats to farms that had $\geq 5$ cats. This finding is consistent with previous reports (Cavalcante et al., 2008; Neto et al., 2008). Cats are the only known definitive host of $T$. gondii, and primary infected cats shed millions of oocysts in the environment (Tenter et al., 2000). The association with the presence of cats is therefore assumed to indicate a causal relationship, and 
limiting the number of cats on goat farms is expected to reduce $T$. gondii infections in goats. In this study, other variables that could potentially increase exposure to cat shed oocysts (e.g., access of cats to the stable, water source, use of silo, use of mixer-feeder, and history of outdoor access) did not show a statistically significant association with $T$. gondii seropositivity. This may have been due to a lack of power. Fifty-two farms participated and 32 animals per farm were tested. With 32 animals tested per farm of 792 goats on average, farms can be misclassified as negative and the maximum possible prevalence at negative farms is $8.8 \%$. This type of misclassification is unlikely to depend on the exposure to risk factors, but can reduce the risk estimates. In addition, with 52 farms, the minimum detectable IR between exposure to risk factors and presence of $T$. gondii at herd level is estimated at 4 , indicating that predictors with a weaker effect were unlikely to be identified as statistically significant in this study.

In conclusion, the serological assay used in this study was suitable for the detection of $T$. gondii antibodies in dairy goats. Indoor-housed Dutch dairy goats were not free from $T$. gondii infection, but the seroprevalence of these indoor kept dairy goats was lower than the published findings in outdoor goats in other European countries. Moreover, the number of cats on the farm was clearly associated with the number of seropositive goats. Since the overall animal level seroprevalence was $13.3 \%$ and a positive relationship between detection of antibodies against $T$. gondii and presence of tissue cysts in meat was found in this species, goats could be a source of $T$. gondii infection for humans. Goat meat and milk should be given a sufficient heat treatment to kill the parasites before consumption. Limiting the presence of cats on the goat farms is expected to reduce the prevalence of $T$. gondii infected goats.

\section{Acknowledgements}

The authors would like to thank Barbara Schimmer (RIVM, Bilthoven, the Netherlands) and Jan van den Broek (Utrecht University, Utrecht, the Netherlands) for their helpful discussions in the preparation of this paper. This research was conducted by a consortium within the framework of project number GA/EFSA/BIOHAZ/2013/01 entitled "Relationship between seroprevalence in the main livestock species and presence of Toxoplasma gondii in meat", grant agreement funded by the European Food Safety Authority (budget). This paper/publication is based on the results obtained in the framework of this mentioned project and it is published under the sole responsibility of the authors, and shall not be considered as an EFSA output. 
Appendix A. Supplementary data

Supplementary data associated with this article can be found, in the online version, at http://dx.doi.org/10.1016/j.prevetmed.2015.12.016. 


\section{REFERENCES}

Antonis, A.F.G., van Knapen, F., Dercksen, D.P., Jager, P.M., 1998. Toxoplasmosis in goats in the Netherlands: a pilot study. Tijdschr Diergeneeskd 123, 561-565.

Bartoń, K., 2014. MuMIn: Multi-Model Inference. http://CRAN.R-project.org/package=MuMIn.

Bokken, G.C.A.M., Bergwerff, A.A., van Knapen, F., 2012. A novel bead-based assay to detect specific antibody responses against Toxoplasma gondii and Trichinella spiralis simultaneously in sera of experimentally infected swine. BMC Vet. Res. 8, doi: 10.1186/1746-6148-1188-1136.

Cavalcante, A.C.R., Carneiro, M., Gouveia, A.M.G., Pinheiro, R.R., Vitor R.W.A., 2008. Risk factors for infection by Toxoplasma gondii in herds of goats in Ceará, Brazil Arq. Bras. Med. Vet. Zootec. 60, 36-41.

Dohoo, I., Martin, W., Stryhn, H., 2009. Veterinary epidemiologic research. VER Inc; 2nd edition.

Dubey, J.P., Rajendran, C., Ferreira, L.R., Martins, J., Kwok, O.C., Hill, D.E., Villena, I., Zhou, H., Su, C., Jones, J.L., 2011. High prevalence and genotypes of Toxoplasma gondii isolated from goats, from a retail meat store, destined for human consumption in the USA. Int. J. Parasitol. 41, 827-833.

Dubey, J.P., Verma, S.K., Ferreira, L.R., Oliveira, S., Cassinelli, A.B., Ying, Y., Kwok, O.C.H., Tuo, W., Chiesa, O.A., Jones, J.L., 2014. Detection and survival of Toxoplasma gondii in milk and cheese from experimentally infected goats. J. Food Protect. 77, 1747-1753.

Garcia-Bocanegra, I., Cabezon, O., Hernandez, E., Martinez-Cruz, M.S., Martinez-Moreno, A., Martinez-Moreno, J., 2013. Toxoplasma gondii in ruminant species (cattle, sheep, and goats) from southern Spain. J. Parasitol. 99, 438-440.

Gebremedhin, E.Z., Agonafi, A., Tessema, T.S., Tilahun, G., Medhin, G., Vitale, M., Marco, V.D., 2013. Some risk factors for reproductive failures and contribution of Toxoplasma gondii infection in sheep and goats of Central Ethiopia: A cross-sectional study. Res. Vet. Sci. 95, 894-900.

Györke, A., Opsteegh, M., Mircean, V., lovu, A., Cozma, V., 2011. Toxoplasma gondii in Romanian household cats: Evaluation of serological tests, epidemiology and risk factors. Prev. Vet. Med. 102, 321-328.

Havelaar, A.H., Haagsma, J.A., Mangen, M.J., Kemmeren, J.M., Verhoef, L.P.B., Vijgen, S.M., Wilson, M., Friesema, I.H., Kortbeek, L.M., van Duynhoven, Y.T.H.P., van Pelt, W., 2012. Disease burden of foodborne pathogens in the Netherlands, 2009. Int. J. Food Microbiol. 156, 231-238.

IBM Corp, 2011. IBM SPSS Statistics for Windows. Armonk, NY: IBM Corp.

lovu, A., Gyorke, A., Mircean, V., Gavrea, R., Cozma, V., 2012. Seroprevalence of Toxoplasma gondii and Neospora caninum in dairy goats from Romania. Vet. Parasitol. 186, 470-474.

Jacobson, R.H., 1998. Validation of serological assays for diagnosis of infectious diseases. Revue scientifique et technique. 17, 469-526.

Jones, J.L., Dubey, J.P., 2012. Foodborne toxoplasmosis. Clin. Infec. Dis. 55, 845-851.

Lopes, A.P., Dubey, J.P., Neto, F., Rodrigues, A., Martins, T., Rodrigues, M., Cardoso, L., 2013. Seroprevalence of Toxoplasma gondii infection in cattle, sheep, goats and pigs from the north of Portugal for human consumption. Vet. Parasitol. 193, 266-269.

Maksimov, P., Buschtöns, S., Herrmann, D.C., Conraths, F.J., Görlich, K., Tenter, A.M., Dubey, J.P., Nagel-Kohl, U., Thoms, B., L., B., 2011. Serological survey and risk factors for Toxoplasma gondii in domestic ducks and geese in Lower Saxony, Germany. Vet. Parasitol. 182, 140-149.

Mancianti, F., Nardoni, S., D'Ascenzi, C., Pedonese, F., Mugnaini, L., Franco, F., Papini, R., 2013. Seroprevalence, detection of DNA in blood and milk, and genotyping of Toxoplasma gondii in a goat population in Italy. BioMed Res. Int. 2013: 905326.

Moraes, E.P.B.S.d., Costa, M.M.d., Dantas, A.F.M., Silva, J.C.R.d., Mota, R.A., 2011. Toxoplasma gondii diagnosis in ovine aborted fetuses and stillborns in the State of Pernambuco, Brazil. Vet. Parasitol. 183, 152-155.

Neto, J.O., Azevedo, S.S., Gennari, S.M., Funada, M.R., Pena, H.F., Araujo, A.R., Batista, C.S., Silva, M.L., Gomes, A.A., Piatti, R.M., Alves, C.J., 2008. Prevalence and risk factors for anti-Toxoplasma gondii antibodies in goats of the Serido Oriental microregion, Rio Grande do Norte state, northeast region of Brazil. Vet. Parasitol. 156, 329-332.

Noordhuizen, J.P.T.M., Thrusfield, M.V., Frankena, K., Graat, E.A.M., 2001. Application of quantitative methods in veterinary epidemiology. Wageningen Pers, 2nd edition Wageningen, NL.

Opsteegh, M., Teunis, P., Mensink, M., Zuchner, L., Titilincu, A., Langelaar, M., van der Giessen, J., 2010. Evaluation of ELISA test characteristics and estimation of Toxoplasma gondii seroprevalence in Dutch sheep using mixture models. Prev. Vet. Med. 96, 232-240.

R Core Team, 2014. R: A language and environment for statistical computing. R Foundation for Statistical Computing, Vienna, Austria.

Reed, G.F., Lynn, F., Meade, B.D., 2002. Use of coefficient of variation in assessing variability of quantitative assays. Clin. Diag. Lab. Immun. 9, 1235-1239. 
Sacks, J.J., Roberto, R.R., Brooks, N.F., 1982. Toxoplasmosis infection associated with raw goat's milk. J. Am. Med. Assoc. 248, 1728-1732.

Schimmer, B., Luttikholt, S., Hautvast, J.L., Graat, E.A., Vellema, P., Duynhoven, Y.T., 2011. Seroprevalence and risk factors of $Q$ fever in goats on commercial dairy goat farms in the Netherlands, 2009-2010. BMC Vet. Res. 7, 81.

Spišák, F., Turčeková, L., Reiterová, K., Špilovská, S., Dubinský, P., 2010. Prevalence estimation and genotypization of Toxoplasma gondii in goats. Biologia. 65, 670-674.

Statistics Netherlands, 2015. Agriculture; crops, livestock and land use by general farm type, region. (http://statline.cbs.nl/StatWeb/publication/). the Netherlands.

Stormoen, M., Tharaldsen, J., Hopp, P., 2012. Seroprevalence of Toxoplasma gondii infection in Norwegian dairy goats. Acta Veterinaria Scandinavica. 54, 75.

Tenter, A.M., Heckeroth, A.R., Weiss, L.M., 2000. Toxoplasma gondii: from animals to humans. Int. J. Parasitol. 30, 1217-1258.

Tzanidakis, N., Maksimov, P., Conraths, F.J., Kiossis, E., Brozos, C., Sotiraki, S., Schares, G., 2012. Toxoplasma gondii in sheep and goats: seroprevalence and potential risk factors under dairy husbandry practices. Vet. Parasitol. 190, 340-348.

van der Giessen, J., Fonville, M., Bouwknegt, M., Langelaar, M., Vollema, A., 2007. Seroprevalence of Trichinella spiralis and Toxoplasma gondii in pigs from different housing systems in the Netherlands. Vet. Parasitol. 148, 371-374.

van Engelen, E., Luttikholt, S., Peperkamp, K., Vellema, P., Van den Brom, R., 2014. Small ruminants abortions in the Netherlands during lambing season 2012-2013. Vet. Rec. doi: 10.1136/vr.102244.

van Knapen, F., Franchimont, J.H., van der Lugt, G., 1982. Prevalence of antibodies to toxoplasma in farm animals in the Netherlands and its implication for meat inspection. Vet. Quart. 4, 101-105.

Vuong, Q.H., 1989. Likelihood ratio tests for model selection and non-nested hypotheses. Econometrica. 57, 307333. 


\section{Chapter}

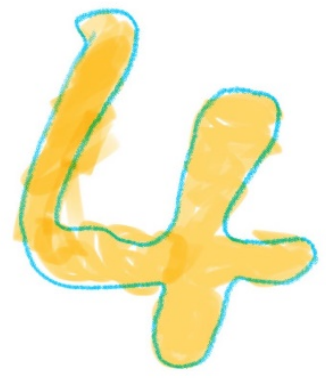

\section{Seroprevalence of Toxoplasma gondii in pregnant women and livestock in the mainland of China: a systematic review and hierarchical meta-analysis}

Scientific Reports, 2018, 8, 6218

Huifang Deng ${ }^{1}$, Brecht Devleesschauwer ${ }^{2}$, Mingyuan $\mathrm{Liu}^{3}$, Jianhua $\mathrm{Li}^{3}$, Yongning $\mathrm{Wu}^{4}$, Joke van der Giessen ${ }^{1}$, Marieke Opsteegh ${ }^{1}$

Authors' affiliations:

${ }^{1}$ Centre for Infectious Disease Control - Zoonoses and Environmental Microbiology, National Institute for Public Health and the Environment, Bilthoven, the Netherlands

${ }^{2}$ Department of Public Health and Surveillance, Scientific Institute of Public Health, Brussels, Belgium

${ }^{3}$ Institute of Zoonosis, Jilin University, Changchun, People's Republic of China

${ }^{4}$ Key Laboratory of China Food Safety Risk Assessment, National Center for Food Safety Risk Assessment, Beijing, People's Republic of China 


\begin{abstract}
Background: Primary Toxoplasma gondii infection in pregnant women may result in abortion, stillbirth, or lifelong disabilities of the unborn child. One of the main transmission routes to humans is consumption of raw or undercooked meat containing $T$. gondii tissue cysts. We aim to determine and compare the regional distribution of $T$. gondii seroprevalence in pregnant women and meat-producing livestock in China through a systematic literature review.
\end{abstract}

Methods: A total of 272 eligible publications were identified from Medline, Scopus, Embase and China National Knowledge Infrastructure. Apparent and true seroprevalence were analysed by region using a novel Bayesian hierarchical model that allowed incorporating sensitivity and specificity of the applied serological assays.

Results: The true seroprevalence of $T$. gondii in pregnant women was $5.0 \%$ or less in seven regions of China. The median of the regional true seroprevalences in pigs (24\%) was significantly higher than in cattle $(9.5 \%)$, but it was not significantly higher than in chickens (20\%) and small ruminants (20\%).

Conclusions: This study represents the first use of a Bayesian hierarchical model to obtain regional true seroprevalence. These results, in combination with meat consumption data, can be used to better understand the contribution of meatproducing animals to human $T$. gondii infection in China. 


\section{Introduction}

Toxoplasma gondii is an obligate intracellular protozoan parasite that causes toxoplasmosis. The parasite is widely distributed in the world and can infect a wide range of warm-blooded animals, including humans, pets and livestock. In the general population, T. gondii infection can remain asymptomatic, cause lymphadenopathy and flu-like symptoms, or lead to eye disease, most frequently chorioretinitis, while in immune-compromised patients, it can be fatal ${ }^{1}$. Pregnant women constitute a specific risk group: if primary infection is acquired during pregnancy, this may lead to abortion, stillbirth and neurological disorders in the unborn child ${ }^{2}$. Congenital and acquired toxoplasmosis caused more than 20 million new cases worldwide in 2010, resulting in an estimated global disease burden of 1.68 million (95\% UI 1.24-2.45 million) disability-adjusted life years (DALYs), of which 829,000 DALYs (95\% UI 561,000-1.26 million) were estimated to be foodborne ${ }^{3}$. In a global multicriteria based ranking (considering public health, animal health, microbial ecology, agribusiness and trade, and socio-economic impact) $T$. gondii ranked fourth out of 24 foodborne parasites ${ }^{4}$.

The main routes of postnatal infection for humans are consumption of raw or undercooked meat containing tissue cysts and food or water contaminated with sporulated oocysts shed by the primary infected definite hosts, felines ${ }^{1}$. As intermediate hosts of $T$. gondii, meat-producing animals serve as one of the main sources of human infections ${ }^{5}$. It was estimated that $30 \%-63 \%$ of infections in pregnant women from six large European cities was attributed to meat ${ }^{6}$. As the effectiveness of treatment is unclear ${ }^{7,8}$, prevention of infection is so far the most important strategy but relies on knowledge of the relative attribution of different transmission routes. Results from an extensive literature review showed that there is a positive relationship between detection of antibodies to $T$. gondii and presence of this parasite in pigs, chickens and small ruminants, but not in cattle and horses ${ }^{9}$. Thus, with the exception of cattle and horses the seroprevalence and geographical distribution of the $T$. gondii infection in different meat-producing animals gives an indication of the risk of human infection via consumption of undercooked meat and is useful for developing health education material for pregnant women and other risk groups. In China, the seroprevalence of $T$. gondii in two national surveys conducted in 1988-1992 and 2001-2004 has increased from 5.2\% to $7.9 \%{ }^{10}$. The geographical distribution of the $T$. gondii seroprevalence in the general population and meat-producing animals is not reported systematically and most of the studies were published in Chinese which are not easily accessible for the international scientific community. Apart from the two national surveys, studies concerning $T$. gondii infection in the general population were hardly available, thus we decided to use data from pregnant women as a proxy for the general population. 
In epidemiological studies true prevalence (TP) rather than apparent prevalence (AP) is the parameter of interest, and requires information on test sensitivity (Se) and specificity (Sp). A variety of serological assays have been developed for the detection of $T$. gondii-specific immunoglobulins ${ }^{11}$ and enzyme-linked immunosorbent assay (ELISA), modified agglutination test (MAT), and indirect hemagglutination antibody test (IHA) are commonly used in China. Unfortunately, estimates of Se and Sp are often lacking or evaluated in the absence of appropriate reference tests or on samples that are not relevant for the target population. Bayesian modelling is therefore increasingly used for veterinary epidemiological studies to infer true prevalence while taking into account the uncertainty of Se and $\mathrm{Sp}^{12-16}$. Furthermore, the Bayesian framework allows explicitly modelling complex hierarchical structures, such as studies nested within regions, which in turn are nested within a country. This has the advantageous side-effect that regions in which few or no studies were performed, can "borrow strength" from the remaining regions, and data gaps can be imputed ${ }^{14,17}$. To our knowledge, however, both applications i.e., true prevalence estimation and hierarchical modelling with data imputation has never been combined in a single model.

The present study estimates the seroprevalence of $T$. gondii in pregnant women and in the main meat-producing animals (i.e., pig, cattle, sheep, goat, chicken, duck, goose and donkey) from different regions of China and analyses the potential link between the seroprevalence in humans and livestock. Data on apparent seroprevalence were collected by systematically reviewing international and Chinese bibliographic databases. A Bayesian hierarchical model that allowed incorporating the sensitivity and specificity of the applied serological assays was used to estimate true prevalence by region and impute possible data gaps.

\section{Methods}

\subsection{Literature review and data sources}

Relevant studies on the seroprevalence of $T$. gondii infection in pregnant women and farm animals in the mainland of China were searched through the Ovid Medline, Scopus, Embase and China National Knowledge Infrastructure (CNKI) electronic databases for English and Chinese publications. Cochrane guidelines and European Food Safety Authority (EFSA) guidance for carrying out systematic reviews were followed for identifying eligible studies 37,38 , and the PRISMA guidelines was followed for reporting ${ }^{39}$. The key elements of this review question were: population (pregnant women and meat-producing animals in China) and outcome ( $T$. gondii seroprevalence). The literature search on Medline, Scopus, Embase was last updated on 20/10/2017, and last updated on CNKI on 18/05/2016. 


\subsection{Search strategy and study selection}

All population-based studies published in the last 16 years (2000-2016) that reported the seroprevalence of anti- $T$. gondii antibodies in Chinese pregnant women or at least one of the animal species of interest were considered for inclusion. There was no restriction on language and the sample size of the study. The combination of "Toxoplasma", "gondii", "toxoplasmosis", "China", and "Chinese" were used as search terms in Medline, Scopus and Embase, the combination of "T. gondii", "toxoplasmosis", "pig", "cattle", "sheep" and "goat", "chicken", "duck", "goose" and "donkey" in Chinese were searched in CNKI. One reviewer (HD), fluent in English and Chinese, read the titles and abstracts of all publications retrieved from the electronic databases and excluded those that clearly did not meet the aforementioned selection criteria. In the next stage, all potential eligible studies were retrieved in full-text and checked by the same reviewer.

Several exclusion criteria were used to select eligible studies: (a) Descriptive studies, reviews, case reports, editorials or letters to the editors without original data, individual animal diagnosis and treatments (epidemiological cross-sectional studies were preferred). (b) Studies not representative for our target population. (c) Animal species not intended for meat consumption (e.g., animals for research purpose). (d) Studies limited to experimental infection with $T$. gondii (rather than natural infection). (e) The outcomes of the studies ( $T$. gondii infection) were not confirmed by a serological assay. (f) Information about the total number of seropositive samples and sample size were not available. (g) Studies not conducted in the mainland of China. (h) Duplicated data.

Information from all relevant studies was extracted and coded into a Microsoft Excel datasheet. Variables extracted included author information, year of publication, animal species, period of data collection, location of the study, diagnostic method, seroprevalence or number of seropositive cases and sample size. If a publication contained multiple studies (e.g., different livestock species or regions), data were extracted separately. For pregnant women, many studies additionally reported the prevalence of IgM antibodies, but only data on IgG seroprevalence of $T$. gondii were included in the analysis.

\subsection{Data analysis}

\subsubsection{Hierarchical meta-analysis model}

The 31 provinces of mainland China were categorized into seven regions, including central, east, north, northeast, northwest, south and southwest of China in our study. Apparent seroprevalence in pregnant women and livestock from different regions were summarized using a three-level hierarchical meta-analysis model. 
First, at the individual study level, the number of seropositive samples $\left(x_{i}\right)$ out of total number of tested samples $\left(n_{i}\right)$ in every single study $i$ was assumed to follow a binomial distribution, equation (1). The logit-transformed seroprevalence $\left(A P_{i}\right)$ of every individual study, conducted in a certain region $j$, was assumed to arise from a normal distribution with a region-specific mean seroprevalence $\left(\theta_{j}\right)$ and within-region variance $\left(\sigma_{w}^{2}\right)$, equation (2). Second, at the regional level, every specific regional seroprevalence was assumed to arise from a normal distribution with the mean of national level seroprevalence $\left(\theta_{0}\right)$ and between-region variance $\left(\sigma_{b}^{2}\right)$, equation (3).

$x_{i} \sim \operatorname{Binomial}\left(A P_{i}, n_{i}\right)$

$\operatorname{logit}\left(A P_{i}\right) \sim \operatorname{Normal}\left(\theta_{j}, \sigma_{w}^{2}\right)$

$\theta_{j} \sim \operatorname{Normal}\left(\theta_{0}, \sigma_{b}^{2}\right)$

After fitting this hierarchical random effects model to the available data, seroprevalence values for regions with no data were imputed based on the resulting posterior predictive distributions. In other words, we represented missing seroprevalence data by distributions based on the fitted mean and variance parameters. For regions where no data were available, the (logittransformed) seroprevalence was imputed as multiple random draws from a normal distribution with mean equal to the fitted national intercept $\theta_{0}$ and variance equal to the fitted between-region variance (thus imputing the seroprevalence as that of a "random" region, with the uncertainty interval describing the variability between regions). The equation was:

$\operatorname{logit}\left(\theta_{j}^{*}\right) \sim \operatorname{Normal}\left(\hat{\theta}_{0}, \hat{\sigma}_{b}^{2}\right)$

\subsubsection{True seroprevalence estimation}

All studies included in the analysis reported apparent seroprevalence instead of true seroprevalence. Therefore, publications about the Se and Sp for each commercial kit used in our selected studies were searched and reviewed. To take the uncertainty of Se and Sp into account, the information was then used by fitting beta distributed priors. The beta distribution is defined on the interval 0-1 and it is very flexible, therefore often used for model probabilities in Bayesian analyses. For those commercial kits which had more than one reported Se and Sp, the numbers of positive sample and tested sample obtained from validation studies were fitted to a random effect meta-analysis similar to the previous hierarchical meta-analysis model but without the multilevel structure. For those commercial kits which had no information at all, they were all categorized as unknown kit. To fit the random effect meta-analysis, data from all the known kits which were as the same type of assay as the unknown kits were used. The parameters of beta distribution were 
then found by using "fitdist" function in the R package "fitdistrplus" with the generated data from the random effect meta-analysis. As initial runs of the Bayesian hierarchical model showed insufficient convergence, beta distributions for the priors of Se and Sp were truncated (Table 3) assuming that the values of Se and $\mathrm{Sp}$ of used kits were at least larger than 0.1. The characteristics of the diagnostic tests ( $S e$ and $S p$ ) were taken into account in equation (6), where $A P_{i}$ is the apparent seroprevalence found in the record and $T P_{i}$ is the true seroprevalence. The equations for the Bayesian hierarchical model were:

$x_{i} \sim \operatorname{Binomial}\left(A P_{i}, n_{i}\right)$

$A P_{i}=S E_{i} * T P_{i}+\left(1-S P_{i}\right) *\left(1-T P_{i}\right)$

$\operatorname{logit}\left(T P_{i}\right) \sim \operatorname{Normal}\left(\pi_{j}, \sigma_{w}^{2}\right)$

$\pi_{j} \sim \operatorname{Normal}\left(\pi_{0}, \sigma_{b}^{2}\right)$

Statistical analyses were performed using $\mathrm{R}$ version 3.3.140. The model was implemented in a Bayesian framework, using independent normal distributed $(0$, 100000) priors for all $\theta_{i}$ and $\theta_{0}$; a uniform distributed $(0,10)$ prior for $\sigma_{w}^{2}$; and a Folded-t (1) distributed prior for $\sigma_{b}^{2}$, as suggested by Gelman ${ }^{41}$. Models were constructed using "rjags" package and data were fitted using Markov Chain Monte Carlo sampling techniques. The models were implemented in $\mathrm{R}$ with the first 10,000 iterations as burn-in and 10,000 iterations as posterior inference. The convergence was checked by visual inspection of density and trace plots, as well as the multivariate potential scale reduction factors (or Brooks-Gelman-Rubin diagnostic). Approximate convergence was diagnosed if the upper confidence limit of the potential scale reduction factor was close to one.

The unit of analysis was set as a single study. Separate analysis was performed for pregnant women and each animal species. The estimates of posterior mean from different regions of China together with the $95 \%$ posterior probability intervals, defined as the distribution's $2.5^{\text {th }}$ and $97.5^{\text {th }}$ percentile, were generated from the analysis. A Kruskal-Wallis test was used to compare the true seroprevalences in different species. If the Kruskal-Wallis test showed a significant result then a posthoc analysis will be performed using Dunn's test and $p$-values were adjusted by "Holm" method for multiple comparisons. The relationship between seroprevalence in pregnant women and meat-producing animals in different regions of China was estimated by checking Spearman's rank correlation coefficient. 


\section{Results}

\subsection{Characteristics of eligible studies}

The selection process of published papers for pregnant women and livestock is summarized in a Preferred Reporting Items for Systematic Reviews and MetaAnalyses (PRISMA) flow diagram as shown in Fig. 1. A total of 72 eligible studies which reported the seroprevalence of IgG antibodies to $T$. gondii for pregnant women were collected from 21 provinces of China; no eligible studies were conducted in Chongqing, Fujian, Hainan, Jiangxi, Neimenggu, Ningxia, Qinghai, Tianjin, Yunnan provinces or the Tibet Autonomous Region (Xizang). The included pregnant women were at different weeks of their pregnancy, and the information of mean age was available from 31 studies, and ranged from 25 to 32 years old.

The literature review identified 200 publications for livestock from 30 provinces. None of the included studies were performed in Tianjin province. A total number of 240 studies were published in these 200 publications. The studies for pregnant women from 21 provinces and for pigs, chickens, cattle and small ruminants from the 30 provinces were merged to seven regions of China (Fig. 2). The entire list of 272 selected studies can be found as Supplementary file S1 online.

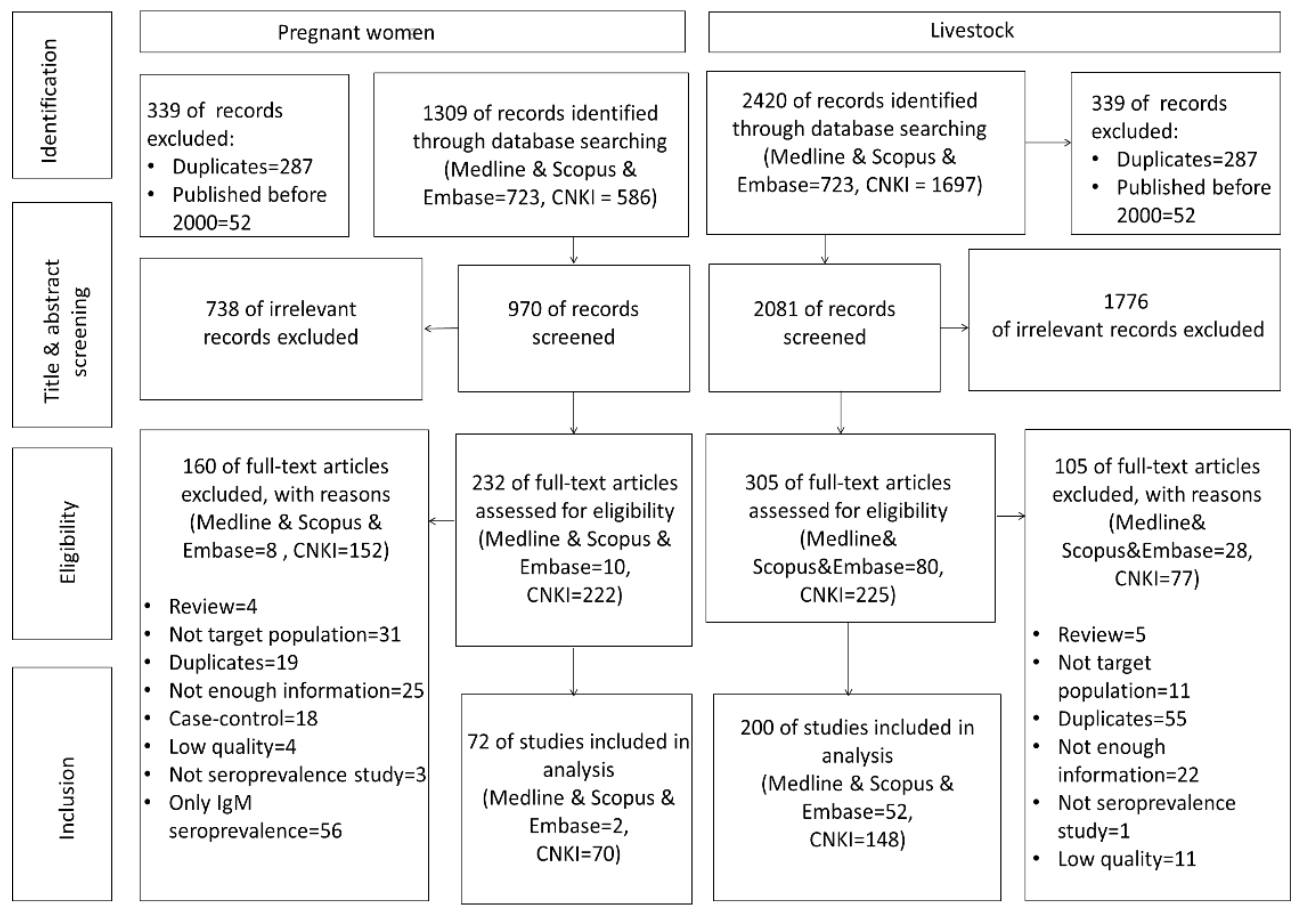

Figure 1. Flowchart: search strategy steps and selection of relevant studies on $T$. gondii seroprevalence in pregnant women and livestock in mainland of China. 


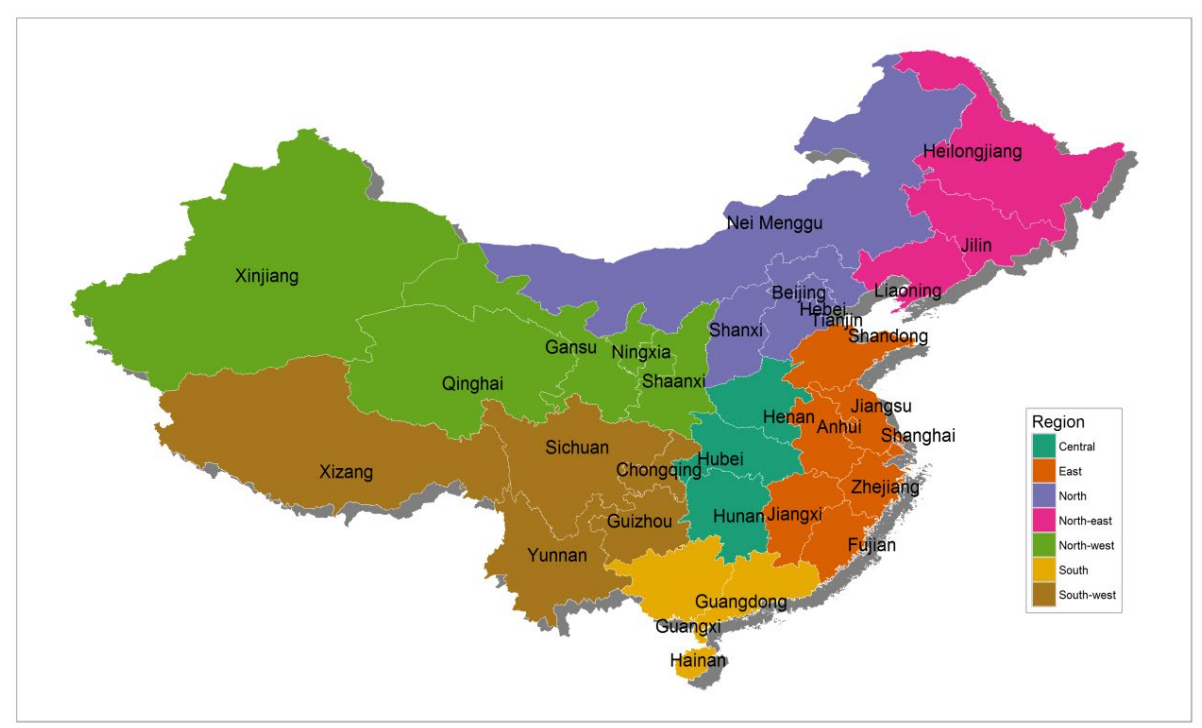

Figure 2. Seven regions from 31 provinces of mainland China.

\subsection{Seroprevalence of pregnant women}

In pregnant women, a small variation of non-modelled apparent prevalence (obtained by summing the data per region) was observed between different regions of China. The non-modelled AP ranged from $2.1 \%$ to $11 \%$ in all seven regions (Table 1). The apparent seroprevalence estimates from the hierarchical model were similar to the results of non-modelled AP. The highest mean AP from the hierarchical model was identified in Northeast $15.7 \%, 95 \%$ PPI (posterior prediction interval) [2.9\%-11\%]), and the lowest in Northwest China $(3.1 \%, 95 \%$ PPI [1.2\%-5.4\%]) (Table 2).

Extracted information of Se and $\mathrm{Sp}$ from the commercial kits used in pregnant women in the selected studies and the parameters of beta distribution used in the Bayesian hierarchical model are shown in Table 3. A total number of 49 out of 72 studies used commercial kits with unknown test characteristics. The mean posterior seroprevalences in Chinese pregnant women and the mean posterior estimates for serological test characteristics are shown in Table $\mathbf{4}$ and $\mathbf{5}$. The Se and $\mathrm{Sp}$ adjusted seroprevalences in Chinese pregnant women ranged from $2.4 \%$ to $5.0 \%$ among different regions. Trace plots and the potential scale reduction factors showed good convergences for the parameters in all models. The codes for the Bayesian hierarchical model can be found as Supplementary file S2 online. 


\subsection{Seroprevalence of livestock}

A wide variation of non-modelled apparent seroprevalence was observed in different animal species (Table 1). The non-modelled AP of $T$. gondii in ducks, goose and donkeys ranged from $9.4 \%-27 \%, 1.7 \%-21 \%$ and $0 \%-24 \%$ respectively. However, since only few studies were eligible from ducks (5), geese (5), and donkeys (5) through the literature review, these animal species were not included in meta-analysis and correlation analysis with pregnant women. Among the other four animal species, the non-modelled AP of $T$. gondii infection was higher in pigs and chickens than in small ruminants and cattle (Table 1). The highest nonmodelled AP was found in pigs (53\%) in Southwest China and the lowest in cattle (5.8\%) in Southwest China. No eligible studies in cattle and small ruminants were conducted in the Southern region of China.

The results of $T$. gondii apparent seroprevalence in different animals from the hierarchical models were similar to the results of non-modelled AP and they are shown in Table 2. The T. gondii modelled AP in small ruminants and cattle in South China were imputed as $9.6 \%$ (95\% PPI, 2.2\%-25\%) and 11\% (95\% PPI, 3.6\%-25\%).

Extracted information of Se and Sp from the commercial kits used in livestock in the selected studies and the parameters of beta distribution used in the Bayesian hierarchical model are shown in Table 3. Among the 240 records, the numbers of records using kit 1-4 were 116, 9, 8 and 26 respectively, 48 studies used ELISA kits with unknown Se and Sp, 33 studies used IHA kits with unknown Se and Sp. The mean posterior estimates of the regional true seroprevalence in pigs (20\%-33\%), chickens (11\%-24\%), small ruminants (15\%-24\%) and cattle $(6.8 \%-12 \%)$ are summarized in Table 4. Wide posterior probability intervals for the sensitivities and specificities were found in some of the included kits used for animals (Table 5). The Kruskal-Wallis test indicated that the median of the regional true seroprevalences in different species was significantly different from each other $(p<0.01)$. The posthoc analysis results showed that the median of the regional true seroprevalences in pigs $(24 \%)$ was significantly higher than in cattle $(9.5 \%)(p<0.05)$ and it was not significantly higher than in chickens $(20 \%)$ and small ruminants $(20 \%)$. Trace plots and the potential scale reduction factors showed good convergences for the parameters in all models.

The results of Spearman's rank correlation coefficient showed that there is no strong relationship between the estimated true seroprevalence in pregnant women and the estimated true seroprevalence in any of the livestock species from the corresponding seven regions of China (Table 6). However, the true seroprevalence in chicken was found strongly correlated with the true seroprevalence in small ruminant (Spearman correlation $=0.86, p=0.01$ ). 


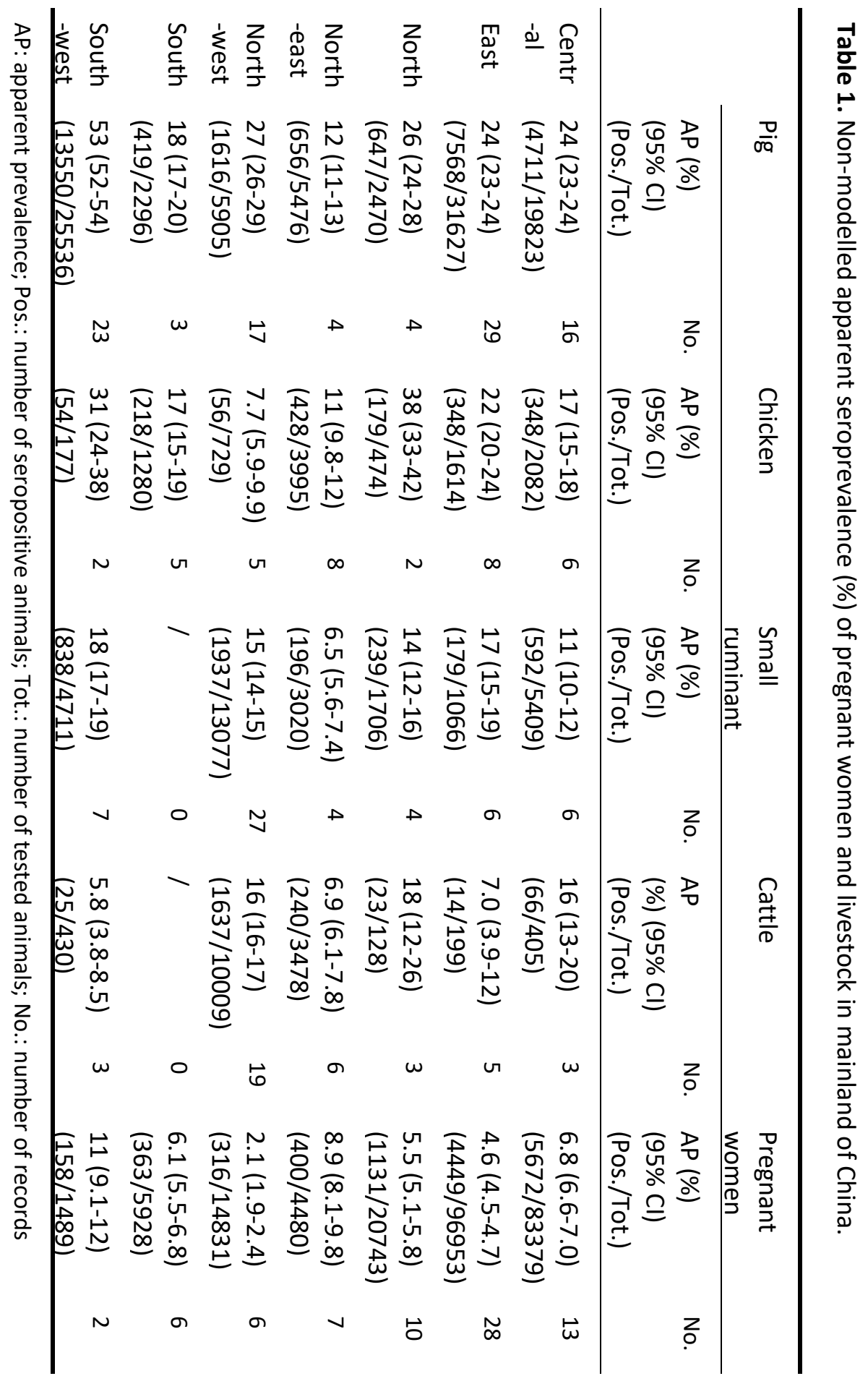




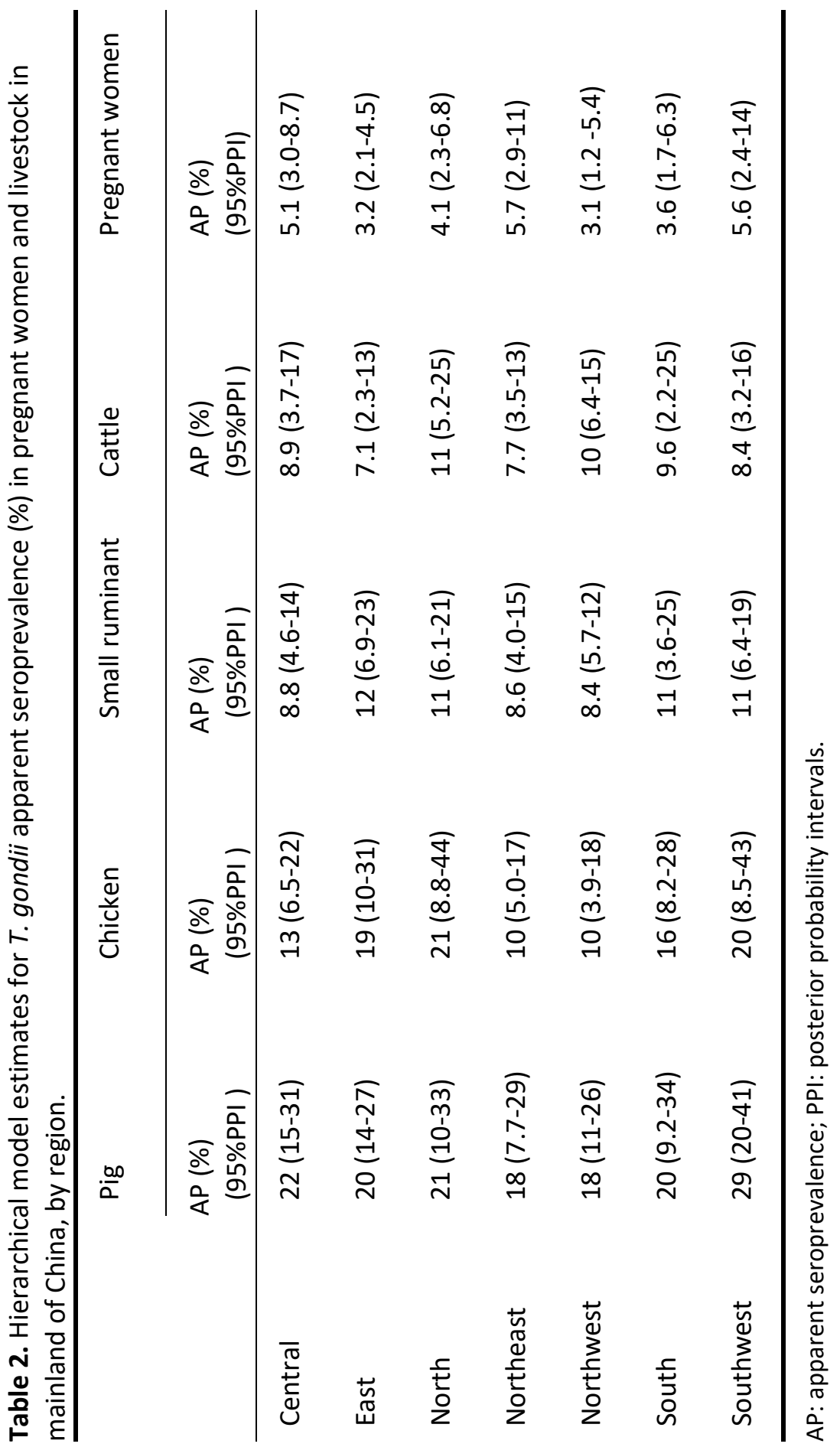




\section{Discussion}

T. gondii causes a high disease burden in humans and is a source of economic losses to livestock industries ${ }^{18,19}$. The increased seroprevalence in $T$. gondii in the Chinese human population together with a notable increase in number of immunecompromised patients suffering cancer and HIV make this opportunistic parasitic disease an important public health challenge to China ${ }^{10,20}$. The aims of our study were to determine the seroprevalence of $T$. gondii in pregnant women and in main meat-producing animals from different regions of China and to examine the potential link between the seroprevalence in humans and livestock.

A total of 72 publications on pregnant women and 200 publications on livestock were included in the dataset. The selected eligible studies for pregnant women covered all seven regions of China. However, data gaps were identified for cattle and small ruminant from Southern China and limited eligible studies were found in ducks, goose and donkeys. These data gaps called for the use of a hierarchical model in which the fitted national (logit-transformed) seroprevalence and the fitted between-region variance could be used to estimate the seroprevalence for regions where no data were available. In all of the eligible publications, seroprevalence data were reported without correction for test sensitivity and specificity. Correctly determining disease status largely depends on the sensitivity and specificity of the serological test. The chance of having a false result can be considerable and the prevalence of test positives may deviate from prevalence of truly infected individuals ${ }^{21}$. For that reason, data on test characteristics were additionally collected from literature. In our study, most of the selected studies dealing with the $T$. gondii IgG seroprevalence in pregnant women and many of the studies on livestock used kits with unknown Se and Sp. Moreover, different values of Se and Sp were reported in literature for the same kit to detect $T$. gondii antibodies in animals. This variation may be due to different characteristics of the reference population (e.g., different levels of exposure to additional pathogens or other biological confounders) and the sampling strategies used in the validation procedures ${ }^{22}$. Thus, it is inappropriate to assume that the test characteristics are constant over populations ${ }^{23}$. Therefore a Bayesian approach to estimate the true seroprevalence of $T$. gondii infection was used. In this approach, prior information about test characteristics was incorporated into the analysis as random variables described using probability distributions in the modelling process. They are therefore not fixed, constant values over different populations. Because insufficient convergence occurred from the initial runs of the model, we used truncated beta distributions for the priors of Se and Sp (Table 3) assuming that the values of Se and Sp of used kits were at least larger than 0.1. After truncation of the distribution of priors, the models for both pregnant women and livestock 
showed good convergence. In general, the Bayesian hierarchical model estimates for $T$. gondii true seroprevalence in pregnant women and livestock were similar to the non-modelled apparent seroprevalence but with wider $95 \%$ posterior probability intervals. In our opinion, the seroprevalences based on the Bayesian hierarchical model provide the most appropriate estimates of seroprevalence, as regional variation and information on test characteristics are taken into account. The uncertainty present due to the use of insufficiently validated diagnostic kits remains unnoticed when apparent prevalences are reported, but is reflected in the wide posterior probability intervals from the Bayesian hierarchical model. Moreover, the Bayesian hierarchical model allowed us to impute seroprevalence estimates for regions that lacked data.

The true seroprevalence from the Bayesian hierarchical model in Chinese pregnant women obtained from this study ranged from $2.4 \%$ to $5.0 \%$ in all seven regions of China. Even though the results were low compared to some countries ${ }^{24}$, this also means that the majority of pregnant women in China are susceptible to a primary infection and their babies to congenital toxoplasmosis. The true seroprevalence was also lower than the overall seroprevalence in Chinese cancer patients reported from a systematic review $(21 \%)^{25}$. This is however not a surprise, as cancer patients are generally older than pregnant women and thus have experienced more time at risk of infection.

The results of true seroprevalence for livestock showed that $T$. gondii infection is wide spread in meat-producing animals in different regions of China. The median of the true seroprevalences from seven regions of China in pigs (24\%) was significantly higher than in cattle (9.5\%), but it was not significantly higher than in chickens (20\%) and small ruminants (20\%). In addition, results showed that the regional variation of true seropevalences in chickens (from $11 \%$ to $24 \%$ ) was strongly correlated with variation in small ruminants (Spearman correlation $=0.86$, $p=0.01$ ). Chickens are considered good indicators of $T$. gondii contamination in the environment, as they become infected mostly by ingesting $T$. gondii oocystscontaminated soil $^{26}$. Therefore, regional variability may indicate variation in environmental contamination with oocysts. In China, sheep is one of the major grazing livestock and they are grazing rotationally by following a predetermined range and routine in pastures ${ }^{27}$. The outdoor access of these animals means that, similarly to chickens, environmental contamination with oocysts is also major risk of infection small ruminants, which may explain the correlation between regional variations of seroprevalence in these species. However, seroprevalence is also strongly associated with farming system with the seroprevalence of $T$. gondii infection higher in outdoor farming systems than in indoor farming system ${ }^{28-30}$. In addition, the age of animals, number of cats present in the farm, and feed source 
are considered as important risk factors associated with $T$. gondii seroprevalence ${ }^{31,32}$. Farming system and exposure to risk factors are likely to also vary by region, and may be correlated for the different species (e.g., in regions with a lot of backyard poultry, backyard farming may also be more common for small ruminants). Therefore, to better understand regional variability in seroprevalence it is important to collect and analyse data taking into account exposure to potential risk factors that are related to $T$. gondii infection in future studies. This type of information was lacking from most of the publications included in the review.

With the exception of cattle and horses, the risk of human $T$. gondii infection via undercooked meat likely increases with a higher seroprevalence in the animal species. Nonetheless, no strong regional relationship between the true seroprevalence in livestock and pregnant women was found. This may be due to the limited data points in our analysis (7 regions). More importantly, the total meat consumption volume and species-specific preparation habits play crucial roles for the relative attribution of different meat-producing animals on a population level ${ }^{33}$. In China, pork is the dominant type of meat consumed by the whole population, except for some religious groups, followed by poultry, beef and lamb, the average annual consumption volumes in 2015 were $20.1,8.4,1.6$ and $1.2 \mathrm{~kg}$ respectively ${ }^{34}$. The highest seroprevalence and total amount of consumption make pork a good potential source of human infections. Additionally, people living in Yunnan, Guizhou and Sichuan provinces eat raw or undercooked pork and beef in their tradition, and the seroprevalence in the ethnic groups from these provinces were found higher than the general population ${ }^{35}$. In our study, the true seroprevalence in pregnant women from the same region was found to be one of the highest $4.8 \%$ (1.7\%-14\%). Due to the diversification of food sources and preparation habits among different regions and ethnic groups, the risk of human infection can vary accordingly. To determine the risk of humans to become infected via the different meat-producing animals, information on prevalence in livestock and meat consumption needs to be combined in a quantitative risk assessment ${ }^{36}$.

In conclusion, the seroprevalence and geographical distribution of $T$. gondii infection in pregnant women and meat-producing animals in China were systematically reviewed and summarized. The results obtained from Bayesian hierarchical models showed that $T$. gondii seroprevalence ranged from $2.4 \%$ to $5.0 \%$ in pregnant women, pigs (20\%-33\%) and chickens (11\%-24\%) had higher true seroprevalence than small ruminants (15\%-24\%) and cattle (6.8\%-12\%). Studies to better evaluate the performance of kits are needed to get more accurate estimates of $T$. gondii seroprevalence in humans and animals. More detailed insight in the geographical distribution of $T$. gondii prevalence in humans and livestock animals 
can be helpful for making effective intervention strategies to reduce the burden of this disease in the Chinese population. However, the risk of meatborne T. gondii infection in humans is not only depending on the prevalence in meat-producing animals but also on consumed volumes and food preparation habits. Therefore the results obtained from this study should be used to determine meatborne toxoplasmosis risk by using a quantitative microbial risk assessment of $T$. gondii infection in China.

\section{Acknowledgements}

This study was carried out as part of the China Exchange Project funded by the Royal Netherlands Academy of Arts and Sciences (530-5CDP21) and the Memorandum of Understanding programme funded by the Ministry of Health, Welfare and Sports in the Netherlands. The authors wish to acknowledge the help of Rob van Spronsen with literature searching and Peter Teunis for useful discussions in the preparation of this paper (both at National Institute for Public Health and the Environment). 


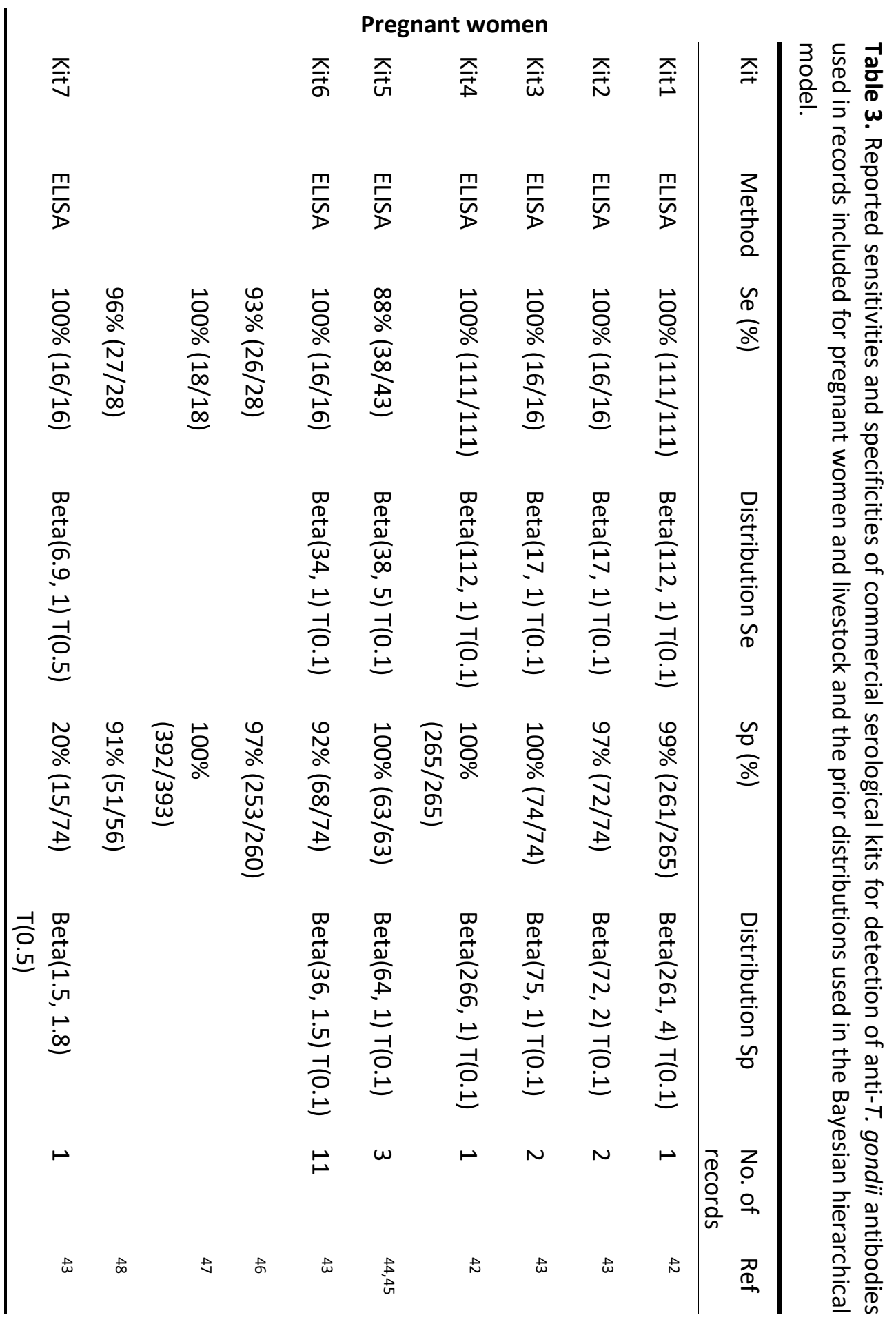




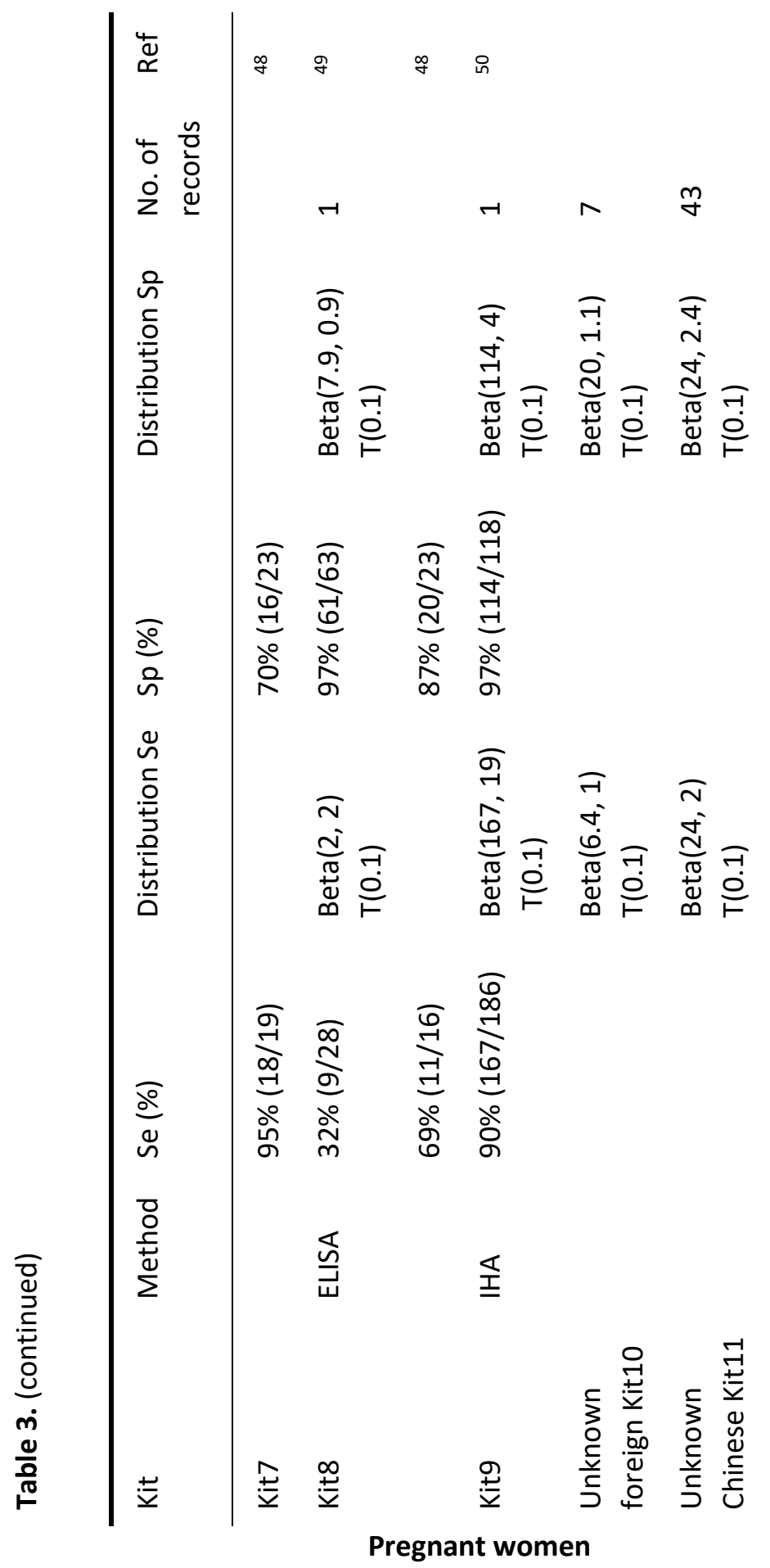


กี กี กี กี กี กี

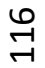

$\infty \quad \infty$

$\stackrel{\infty}{\sim} \stackrel{\infty}{\sim} \stackrel{m}{m}$

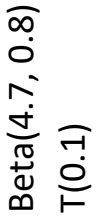

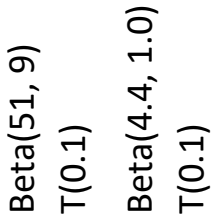

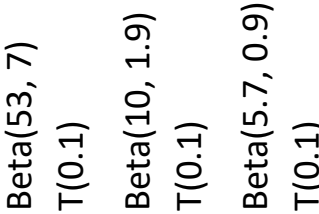

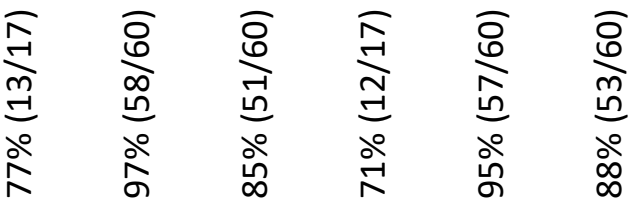

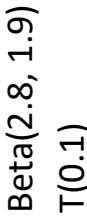

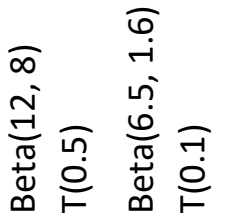

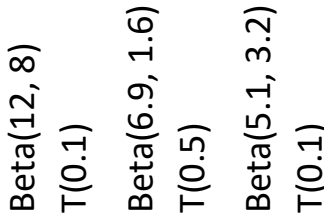

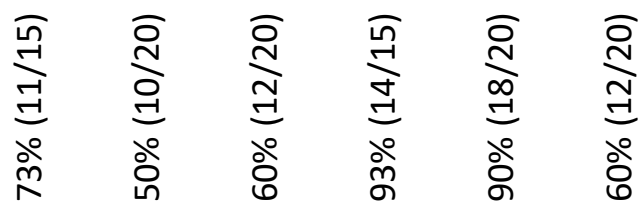

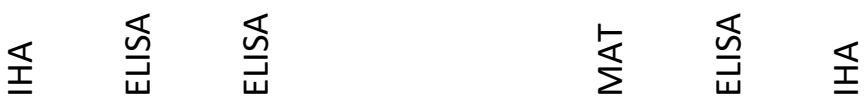

$\stackrel{ㅍ}{ \pm}$

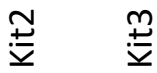

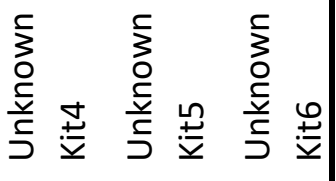

Livestock 


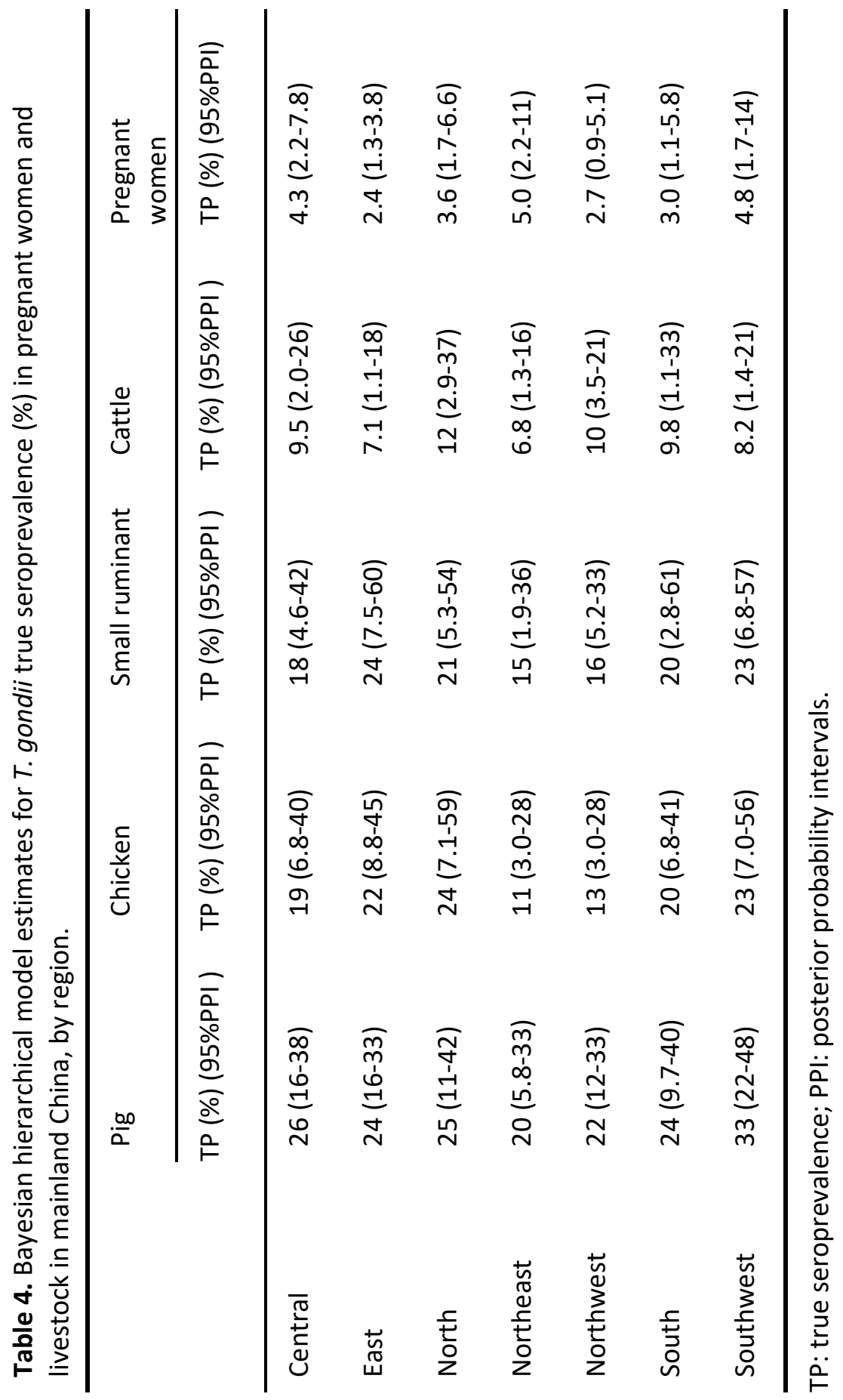


Table 5. Bayesian hierarchical model estimates for sensitivities and specificities of commercial serological kits for detection of anti- $T$. gondii antibodies in pregnant women and livestock.

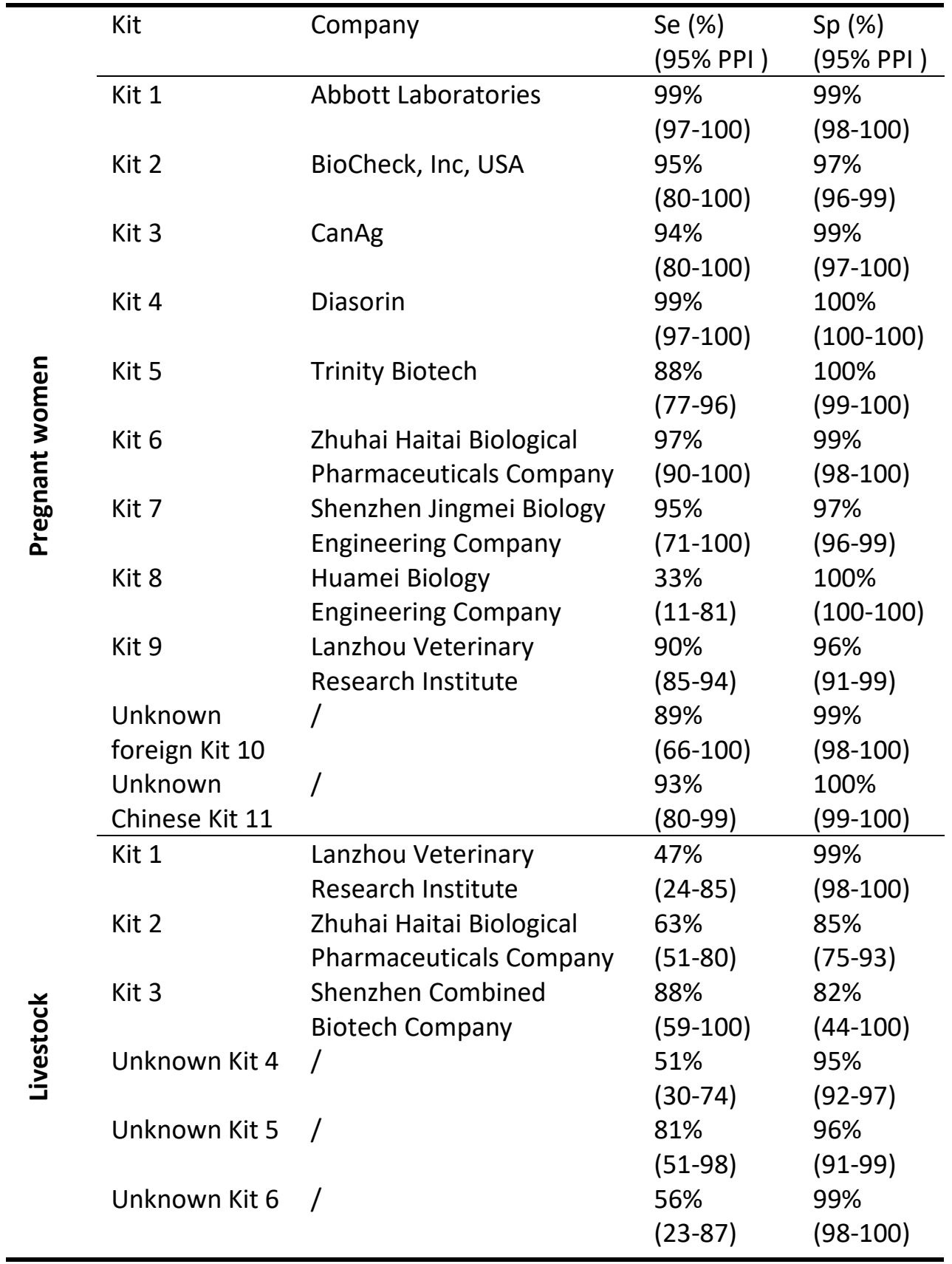


Table 6. Correlation of regional $T$. gondii true seroprevalence between pregnant women and livestock in mainland of China

\begin{tabular}{lllllll}
\hline & & Pig & Chicken & $\begin{array}{l}\text { Small } \\
\text { ruminant }\end{array}$ & Cattle & $\begin{array}{l}\text { Pregnant } \\
\text { women }\end{array}$ \\
\hline Pig & Spearman's $\rho$ & 1 & & & & \\
Chicken & $\begin{array}{l}\text { Spearman's } \rho \\
\text { p-value }\end{array}$ & 0.70 & 1 & & & \\
& & 0.08 & & & & \\
Small & Spearman's $\rho$ & 0.59 & 0.86 & 1 & & \\
ruminant & $p$-value & 0.16 & $\mathbf{0 . 0 1}$ & & & \\
Cattle & $\begin{array}{l}\text { Spearman's } \rho \\
\text { p-value }\end{array}$ & 0.20 & 0.36 & 0 & 1 & \\
& & 0.67 & 0.43 & 1 & & \\
$\begin{array}{l}\text { Pregnant } \\
\text { women }\end{array}$ & $\begin{array}{l}\text { Spearman's } \rho \\
p \text {-value }\end{array}$ & 0.18 & -0.18 & -0.39 & -0.35 & 1 \\
& & 0.70 & 0.70 & 0.38 & 0.43 & \\
\hline
\end{tabular}




\section{REFERENCES}

Tenter, A. M., Heckeroth, A. R. \& Weiss, L. M. Toxoplasma gondii: from animals to humans. Int. J. Parasitol. 30, 1217-1258 (2000).

Torgerson, P. R. \& Mastroiacovo, P. The global burden of congenital toxoplasmosis: a systematic review. Bull. World Health Organ. 91, 501-508 (2013).

Torgerson, P. R. et al. World Health Organization estimates of the global and regional disease burden of 11 foodborne parasitic diseases, 2010: a data synthesis. PLoS Med. 12, e1001920, (2015).

FAO/WHO. Multicriteria-based ranking for risk management of food-borne parasites. http://www.fao.org/publications/card/en/c/ee07c6ae-b86c-4d5f-915c-94c93ded7d9e/ (2014).

\section{Tenter, A. M. Toxoplasma gondii in animals used for human consumption. Mem. Inst. Oswaldo Cruz.} 104, 364-369 (2009).

Cook, A. J. et al. Sources of toxoplasma infection in pregnant women: European multicentre casecontrol study. BMJ 321, 142-147 (2000).

Gilbert, R. E. et al. Effect of prenatal treatment on mother to child transmission of Toxoplasma gondii: retrospective cohort study of 554 mother-child pairs in Lyon, France. $\quad$ Int. J. Epidemiol. 30, 1303-1308 (2001).

Montoya, J. G. \& Liesenfeld, O. Toxoplasmosis. Lancet 363, 1965-1976, (2004) .

Opsteegh, M. et al.Relationship between seroprevalence in the main livestock species and presence of Toxoplasma gondii in meat (GP/EFSA/BIOHAZ/2013/01) An extensive literature review. Final report. EFSA supporting publication 2016:EN-996; https://www.efsa.europa.eu/en/supporting/pub/996e (2016).

Ji, Z., Xue, S., Ji, Y. \& Zhi, B. A national survey on current status of the important parasitic diseases in human population. Chin. J. Parasitol. Parasit. Dis. 23, 332-340 (2005).

1 Liu, Q., Wang, Z. D., Huang, S. Y. \& Zhu, X. Q. Diagnosis of toxoplasmosis and typing of Toxoplasma gondii. Parasit. Vectors 8, 292 (2015).

McAloon, C. G. et al. Bayesian estimation of prevalence of paratuberculosis in dairy herds enrolled in a voluntary Johne's Disease Control Programme in Ireland. Prev. Vet. Med. 128, 95-100 (2016).

Beauvais, W., Orynbayev, M. \& Guitian, J. Empirical Bayes estimation of farm prevalence adjusting for multistage sampling and uncertainty in test performance: a Brucella cross-sectional serostudy in southern Kazakhstan. Epidemiol. Infect. 9, 1-9 (2016).

4 McDonald, S. A. et al. Data-driven methods for imputing national-level incidence in global burden of disease studies. Bull. World Health Organ. 93, 228-236 (2015).

Hanson, T., Johnson, W. O. \& Gardner, I. A. Hierarchical models for estimatingherd prevalence and test accuracy in the absence of a gold standard. J. Agric. Biol. Environ. Stat. 8, 223-239 (2003).

6 Branscum, A. J., Gardner, I. A. \& Johnson, W. O. Bayesian modeling of animal- and herd-level prevalences. Prev. Vet. Med. 66, 101-112 (2004).

Devleesschauwer, B. et al. Methodological framework for World Health Organization estimates of the global burden of foodborne disease. PloS One 10, e0142498 (2015).

Torgerson, P. R. et al. The global burden of foodborne parasitic diseases: an update. Trends Parasitol. 30, 20-26 (2014).

Cenci-Goga, B. T., Rossitto, P. V., Sechi, P., McCrindle, C. M. \& Cullor, J. S. Toxoplasma in animals, food, and humans: an old parasite of new concern. Foodborne Pathog. Dis. 8, 751-762 (2011).

Yang, G. et al. Rapid health transition in China, 1990-2010: findings from the Global Burden of Disease Study 2010. Lancet 381, 1987-2015 (2013).

1 Speybroeck, N., Devleesschauwer, B., Joseph, L. \& Berkvens, D. Misclassification errors in prevalence estimation: Bayesian handling with care. Int. J. Public Health 58, 791-795 (2013).

Greiner, M. \& Gardner, I. A. Epidemiologic issues in the validation of veterinary diagnostic tests. Prev. Vet. Med. 45, 3-22 (2000).

3 Berkvens, D., Speybroeck, N., Praet, N., Adel, A. \& Lesaffre, E. Estimating disease prevalence in a Bayesian framework using probabilistic constraints. Epidemiology 17, 145-153 (2006).

4 Pappas, G., Roussos, N. \& Falagas, M. E. Toxoplasmosis snapshots: global status of Toxoplasma gondii seroprevalence and implications for pregnancy and congenital toxoplasmosis. Int. J. Parasitol. 39, 1385-1394 (2009).

5 Jiang, C., Li, Z., Chen, P. \& Chen, L. The seroprevalence of Toxoplasma gondii in Chinese population with cancer: A systematic review and meta-analysis. Medicine 94, e2274, (2015). 
Ruiz, A. \& Frenkel, J. K. Intermediate and transport hosts of Toxoplasma gondii in Costa Rica. Am. J. Trop. Med. Hyg. 29, 1161-1166 (1980).

$\mathrm{Hu}$, Z. \& Zhang, D. The pasture/forage resource profile for China. http://www.fao.org/ag/agp/agpc/doc/counprof/PDF\%20files/China.pdf (2006).

van der Giessen, J., Fonville, M., Bouwknegt, M., Langelaar, M. \& Vollema, A. Seroprevalence of Trichinella spiralis and Toxoplasma gondii in pigs from different housing systems in the Netherlands. Vet. Parasitol. 148, 371-374 (2007).

Maksimov, P. et al. Serological survey and risk factors for Toxoplasma gondii in domestic ducks and geese in Lower Saxony, Germany. Vet. Parasitol. 182, 140-149 (2011).

Guo, M. et al. A systematic meta-analysis of Toxoplasma gondii prevalence in food animals in the United States. Foodborne Pathog. Dis. 13, 109-118 (2016).

Deng, H. et al. Risk factors related to Toxoplasma gondii seroprevalence in indoor-housed Dutch dairy goats. Prev. Vet. Med. 124, 45-51 (2016).

Guo, M. et al. Prevalence and risk factors for Toxoplasma gondii infection in meat animals and meat products destined for human consumption. J. Food Prot. 78, 457-476 (2015).

Jones, J. L. et al. Risk factors for Toxoplasma gondii infection in the United States. Clin. Infect. Dis. 49, 878-884 (2009).

National Bureau of Statistics of China. Statistical yearbooks. http://www.stats.gov.cn/tjsj/ndsj/2016/indexeh.htm (2016).

Zhou, P. et al. Toxoplasma gondii infection in humans in China. Parasit. Vectors 4, 165 (2011).

Opsteegh, M., Prickaerts, S., Frankena, K. \& Evers, E. G. A quantitative microbial risk assessment for meatborne Toxoplasma gondii infection in The Netherlands. Int. J. Food Microbiol. 150, 103-114 (2011). EFSA. Application of systematic review methodology to food and feed safety assessments to support decision making. EFSA Journal 8, 1637 (2010).

Higgins, J. P. T., \& Green S. Cochrane handbook for systematic reviews of interventions. www.handbook.cochrane.org (2011).

Moher, D. et al. Preferred reporting items for systematic review and meta-analysis protocols (PRISMAP) 2015 statement. Syst. Rev. 4, 1, (2015).

R: A Language and Environment for Statistical Computing (R Foundation for Statistical Computing, Vienna, Austria, 2006).

Gelman, A. Prior distributions for variance parameters in hierarchical models. Bayesian Anal. 1, 515533 (2006).

Calderaro, A. et al. Evaluation of Toxoplasma gondii immunoglobulin G ( $\lg G)$ and $\operatorname{lgM}$ assays incorporating the newVidia analyzer system. Clin. Vaccine Immunol. 15, 1076-1079 (2008).

He, Y., Jiang, S., Ma, X. \& Qiu, Q. Evaluation of Sixteen kinds of kits inner and abroad available for detecting antibodies of Toxoplasma gondii. Science of Travel Medicine 14, 43-45 (2008).

Trinity Biotech. Captia ${ }^{\text {TM }}$ Toxoplasma gondii IgG. http://documents.trinitybiotech.com/product\%20documents/2325100-29\%20EN.pdf (2015)

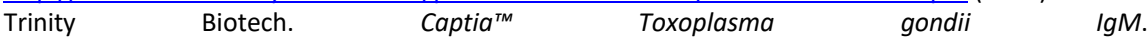
http://documents.trinitybiotech.com/product\%20documents/2325160-29\%20EN.pdf (2015)

Duan, J. \& Zhuang, S. Compare the results of Chinese and imported kits for detection of Toxoplasma gondii IgG antibodies. Chin. J. Zoonoses 19, 127 (2003).

Wang, W., Xu, S. \& Xia, J. Evaluation of one ELISA kit for detection of Toxoplasma gondii IgG antibodies. Central China Medical Journal 31, 229-231 (2007).

Yu, E. \& Gan, S. Re-evaluation of several test kits for detecting Toxoplasma gondii antibodies. Chin. J. Zoonoses 17, 5-6 (2001).

Jiang, S., Zhang, S., Pan, C., He, Y. \& Wei, M. Evaluation of five commercial available kits for detecting antibodies of Toxoplasma gondii. Chin. J. Zoonoses 19, 97-99 (2003).

Yang, Y., Chen, Y., Wei, S. \& Song, R. Efficiency of three methods for detecting Toxoplasma IgG antibody. Chin. J. Schisto. Control 26, 109-110 (2014).

Luo, C., You, J., Liu, J. \& Huang, C. Comparison of ELISA and IHA for detecting serum antibody against Toxoplasma gondii in Swine. China Animal Health 14, 31-34 (2012).

Liu, S. et al. Comparison of several methods for detection of IgG against Toxoplasma gondii. Chinese J. Vet. Med. 48, 72-74 (2012). 


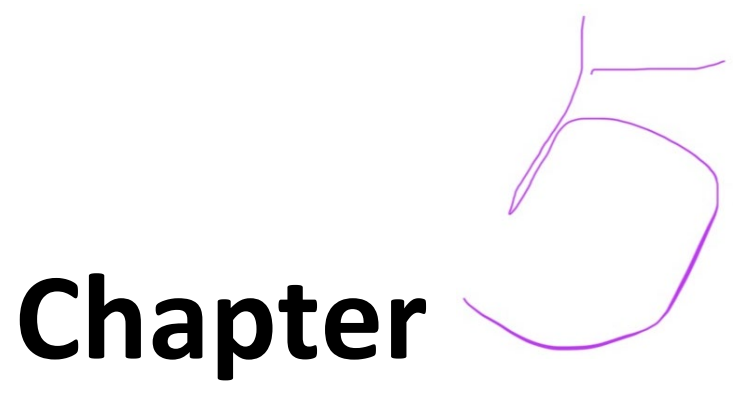

\section{The effect of salting on Toxoplasma gondii viability evaluated and implemented in a quantitative risk assessment of meat-borne human infection}

International Journal of Food Microbiology, 2020, 314, 108380

Huifang Deng, Arno Swart, Axel A. Bonačić Marinović, Joke W. B. van der Giessen, Marieke Opsteegh

Authors' affiliations:

Centre for Infectious Disease Control - Zoonoses and Environmental Microbiology, National Institute for Public Health and the Environment, Bilthoven, the Netherlands 


\begin{abstract}
Background: The protozoan parasite Toxoplasma gondii can infect all warmblooded animals and it causes the disease toxoplasmosis. Meat containing viable $T$. gondii tissue cysts is considered one of the main sources of human infection. The relative importance of the different types of meat depends, not only on the prevalence of $T$. gondii infection in the different livestock species, but also on consumed volumes and preparation habits. To take these factors into account and to estimate the relative contribution of different meat products to human infection, a quantitative risk assessment model for meat-borne $T$. gondii infection was previously developed. However, at the time, the effect of salting on parasite viability was estimated based on a single experiment. In recent years, data using salting methods that are more in line with processing of meat products have come available.
\end{abstract}

Methods: Literature data on the effect of salting on $T$. gondii viability were collected and used to fit a predictive model. In addition to the new salting model, a lower concentration of bradyzoites in cattle, more specific heating profiles, and more recent consumption data were implemented in the QMRA model for meatborne $T$. gondii infection in the Netherlands.

Results: Results show that beef remains the most important source, as it contributed $84 \%$ of the total number of predicted infections in the Dutch population, followed by pork (12\%), mutton (3.7\%), lamb $(0.2 \%)$ pork/beef mixed products $(0.1 \%)$, and veal $(0.01 \%)$. The predicted number of $T$. gondii infections is reasonably in line with epidemiological data. At the product level, filet americain (a raw beef spread) alone contributed $80 \%$ of the total predicted infections in the base model, but scenario analyses demonstrate that its contribution is highly dependent on the salting parameters.

Conclusions: A clear identification of the most risky meat products is important, as interventions focussing on these products could have a great impact on reducing T. gondii disease burden in the Netherlands. For that reason, it is important that the effects of salting and other processing methods are evaluated in line with industrial processing and incorporated in quantitative risk assessment models for meat-borne toxoplasmosis. 


\section{Introduction}

Toxoplasmosis is a zoonotic disease caused by the single-celled parasite Toxoplasma gondii. Although most infections are asymptomatic in immunocompetent individuals, the infection can cause severe health problems in pregnant women and immunocompromised patients (Montoya and Liesenfeld, 2004). In the Netherlands, it was estimated that $26 \%$ of the general population is seropositive (Hofhuis et al., 2011) and the incidence of congenital toxoplasmosis was estimated at 2 cases per 1,000 live-born children (Kortbeek et al., 2009). In addition, $T$. gondii is associated with one of the highest disease burdens among fourteen food-related pathogens in the Netherlands (Havelaar et al., 2012; Mangen et al., 2015; Mangen et al., 2017).

Humans acquire $T$. gondii infection through consumption of raw or undercooked meat containing viable tissue cysts, through ingestion of oocysts in soil, water or contaminated fruits and vegetables (Tenter et al., 2000). However, it is not yet clear which of those two parasite stages and the associated transmission routes are more important to human infections. To date, several risk assessments concerning the exposure/risk of meat-borne $T$. gondii infection to different populations have been published. Among these, there are two qualitative assessments for meat products (Guo et al., 2015; Mie et al., 2008). The first quantitative risk assessment model (QMRA) focused on the relative atrribution of beef, pork and sheep products to human infection in the Netherlands (Opsteegh et al., 2011a). Afterwards, one quantitative model that focused on lamb (Guo et al., 2016b), three QMRAs that focused on the risk from pork (Condoleo et al., 2017; Crotta et al., 2017; Guo et al., 2017), and one that focussed on beef and pork (Belluco et al., 2018) were published. Among those studies, a scaling factor for animal-derived dose-response model was computed and implemented to predict human T. gondii infections (Guo et al., 2016a; Guo et al., 2017) , and also applied by Condoleo et al. (2017). In addition, a probabilistic model to assess the exposure to $T$. gondii through fresh pork meat in England has been developed, where tissue cyst instead of bradyzoites was used as biological unit to which consumers are exposed (Crotta et al., 2017). Both studies that included several livestock species in one model (Belluco et al., 2018; Opsteegh et al., 2011a) concluded that beef plays a more significant role in human $T$. gondii infection than other meat types. Only a few studies took the effect of salting into account (Condoleo et al., 2017; Guo et al., 2017; Opsteegh et al., 2011a). In those studies, logistic regressions were applied to calculate $T$. gondii survival after salting. Briefly, the probability of obtaining an infection from salt-treated meat was calculated using a logistic regression with salting concentration, duration and temperature as parameters (Guo et al., 2017; Opsteegh et al., 2011a), which was based on one major bioassay experiment (Dubey, 1997). More recently, studies describing the survival of $T$. 
gondii tissue cysts after salt treatments became available and results suggest that $T$. gondii might be more sensitive to salt than previously demonstrated (Genchi et al., 2017; Pott et al., 2013). In some of these studies, the salting method is more in line with the processes used in industry. Therefore, we aimed to re-evaluate the effect of salting including all literature currently available.

In the previously reported QMRA to estimate the number of new meat-borne $T$. gondii infections per year in the susceptible Dutch general population and among susceptible pregnant women (Opsteegh et al., 2011a), consumption data on beef, sheep and pork products from the 1998 Dutch National Food Consumption Survey were used (DNFCS, 1998). However, consumption patterns may have changed in 20 years. Therefore, in addition to incorporating the more recent data from literature results of salting, also more recent food consumption data (DNFCS, 2010) were implemented in combination with more specific data on Dutch consumption habits (e.g., cooking temperature) that have been collected for QMRA purposes (Evers et al., 2017). As the concentration of bradyzoites in beef was found to be at least a hundred times lower in cattle (Opsteegh et al., 2011b) than in sheep (Opsteegh et al., 2010a), a lower bradyzoite concentration was used for beef.

\section{Material and methods}

\subsection{Model structure}

The QMRA model structure was used as previously described (Opsteegh et al., 2011a). Briefly, it first models the change in number of viable $T$. gondii bradyzoites in meat product portions due to different processing methods such as salting, freezing and heating and then the probability of human infection. Previously, only freezing by consumers was considered in the model, now an additional freezing step at producer level was added. The outcomes of the model are the predicted annual number of new infections and the relative contributions of different meat products to the total predicted infections in the general population and pregnant women in the Netherlands.

\subsection{The change in number of bradyzoites in infected portions}

\subsubsection{Selection of meat products and consumption data}

The consumption of meat (frequency and quantity) for the Dutch general population was estimated using data from the Dutch National Food Consumption Survey in 2007-2010 (DNFCS, 2010). In the survey, 3,819 (response rate: 69\%) persons aged 7 to 69 years completed two non-consecutive 24-hour dietary recalls per person. For each meat product, the total number of eating occasions and the quantities (without bones) were recorded. Demographic data such as age, sex, and location were collected. 
In the meat consumption dataset, each product has been assigned to a meat type (beef, lamb, mutton, pork, veal, and beef/pork mixed). Similar to the previous QMRA study, poultry products were left out as the prevalence of $T$. gondii infection is low and proper heating is applied. In addition, some of the meat products in the Netherlands are processed or manufactured using different treatments before being placed into the market. Thus, a selection of meat products based on a preretail table from Evers et al. (2017) (supplementary material), and a guide for the production of meat products has been conducted (van Trigt, 2012). First, all processed products were categorized into nine groups according to Evers et al. (2017). Then, products heated to a temperature that surely inactivates $T$. gondii (core temperature $>65^{\circ} \mathrm{C}$ ) (Dubey et al., 1990), and canned products were excluded from the dataset.

The gamma distribution has as support from zero to infinity, and can be flexibly parametrised using two parameters. Therefore, gamma distributions were fitted to the recorded quantities from the Dutch National Food Consumption Survey to obtain a portion size distribution for every product. For products which had insufficient number of eating occasions to fit a gamma distribution, they were regrouped with similar products and fitted with gamma distributions again (details in supplementary material). Multiplications according to Opsteegh et al. (2011a) were used to calculate the total number of portions consumed per year in the Dutch general population and pregnant women.

\subsubsection{Lower bradyzoite concentration in unprocessed beef}

For the concentration of bradyzoites in infected meat, real-time PCR results from naturally infected sheep hearts were used (Opsteegh et al., 2010a). We assume that the $\log _{10}$-transformed concentration of bradyzoites per 100 grams of meat are distributed according to a beta-general distribution (shape parameters 6.5 and 5.7, minimum 0, and maximum 6.8 were used, as in the previous QMRA study (Opsteegh et al., 2011a)). Such a beta-general distribution gives great flexibility of modelling a wide range of shapes and supports. The mean value of the betageneral distribution was 3.6, which corresponds to 3,981 bradyzoites per 100 grams in meat. The same concentration was used for all products except beef or veal and pork/beef mixed products. Studies showed that the concentration of $T$. gondii bradyzoite was at least 100 times lower in cattle than in sheep (Opsteegh et al., 2011b; Opsteegh et al., 2016b). Therefore, bradyzoites concentration was set to be 100 times less in beef products than in other meat products in this QMRA model. Bradyzoites concentrations in pork/beef mixed products decreased correspondingly. 
The number of bradyzoites per unprocessed infected portion per product was calculated by multiplying the number of bradyzoites per gram and the weight of portion size drawn from the beta-general distribution, and the gamma distribution respectively.

\subsubsection{Viable bradyzoite concentration after freezing, salting and cooking}

The number of $T$. gondii cannot grow after the death of the host, but processing of meat can reduce the number of viable bradyzoites in contaminated meat. Therefore, the effects of inactivation of $T$. gondii from freezing and salting at producers together with freezing and heating at consumers' kitchens were taken into account in the analysis. The number of viable bradyzoites in infected meat products after all processed steps were calculated by multiplying the number of bradyzoites in unprocessed portions obtained from the previous step with reduction factors for freezing, salting, and heating. The reduction factors were calculated by logistic regression models based on two mouse bioassay experiments for freezing (Kotula et al., 1991) and heating (Dubey et al., 1990) together with the reversed dose-response relation in mice as reported by Opsteegh et al. (2011a).

A new generalized linear mixed effects model for salting was fitted to data from several studies. A search of publications concerning the effect of salting on inactivation of $T$. gondii in meat was carried out through PubMed. "Toxoplasma gondii" and "salting" were used as search terms and there was no restriction on search period, language and geographic location. All potential eligible studies were retrieved in full-text and checked. References from reviews focused on T. gondii inactivation were checked for additional studies (snowball method). Information about the salting concentration, duration, temperature, salting method, type of tissue used in the experiment, use of sodium nitrite, bioassay animal (cat/mouse), number of infected/non-infected animals, and total number of experimental animals were collected from selected relevant studies (supplementary material). Data points without salting concentration $(\mathrm{NaCl})$, with salting temperature lower than $0{ }^{\circ} \mathrm{C}$, and salting duration equal to 0 day were excluded from the analysis. The number of infected bioassay animals and total number of animals were used as dependent variables and all the other parameters (salting concentration, duration, temperature, salting method, type of tissue used in the experiment, use of sodium nitrite, and bioassay animal) were evaluated as independent variables in the mixed model. Further, univariate associations between the dependent variable and each independent variable were estimated and biologically sound interactions were tested. All independent variables with a $p$ value less than 0.05 from the univariate analysis were selected and were put in the multivariate model. In the full model, all the selected independent variables were set as fixed effects and individual study was set as a random effect. The random effect was used to take the differences 
between studies into account and to control the unobserved heterogeneity. A stepwise selection was performed and parameter selection was based on Akaike information criterion (AIC). The model with the lowest AIC value was considered the best fit for explaining the data. When the difference of AIC values between two models was less than two, then the one with less independent variables was selected. Residuals of the fitted model were visually checked for normality and conditional coefficient $R^{2}$ (variance explained by the entire model, including fixed and random effects) was checked for the goodness of fit. To use the fitted mixed model for calculating the reduction factor for salting for every salted meat product, input values of the parameters in the model (salt concentration, salting method, duration, temperature, and use of sodium nitrite) were assigned based on a Dutch manual for the production of meat products (van Trigt, 2012).

Besides salting, the following sections of the model were also modified compared to the previous study: first, some meat products may be prepared from frozen meat (mainly minced products, e.g., sausage, filet americain, and hamburger); therefore, the effect of freezing at producer level was added. In the dataset, every product was assigned to a fraction $(0,0.5,0.75,0.9)$ of being frozen at producer (based on personal communication with the meat industry and van Trigt, (2012), parameter values per product are provided in supplementary material), and this fraction was used in a binomial distribution to determine if the product was made from frozen meat or not. The temperature of storage at producers was assumed uniformly distributed at -18 and $-24{ }^{\circ} \mathrm{C}$ (based on international four-star rating system for freezers). In addition, the effect of freezing was also applied at consumers' homes, but with different fractions of being placed in the freezer. The temperatures were based on international four-star rating system for freezers (-6, $-12,-18$, and $\left.-24{ }^{\circ} \mathrm{C}\right)$. Products were classified into four groups ((1) meat to be cooked; (2) shelf-stable meat products; (3) meat products to be consumed raw; (4) perishable meat products) with different fractions of portions stored in consumers' freezers $(0.56,0.01,0.03$, and 0.02) (Chardon and Swart, 2016) (supplementary material). A uniform distribution ranging from 24 to 720 hours was used for the duration of freezing at both producers and consumers (same as in the previous QMRA study (Opsteegh et al., 2011a)). Second, for heating, instead of using cooking temperature data from the Food and Drug Administration from United States (FDA, 1999), which only specified a temperature distribution for intact and minced meat products, heating temperature profiles were used to fit beta-general distributions in the analysis (Evers et al., 2017). Ten different heating temperature profiles were used, and the most suitable profile was assigned to each product included in the model based on the products characteristics (e.g., animal species and cut) (Table 1). 
Table 1. Heating temperature profiles at consumers' home fitted with beta-general distributions (Evers et al., 2017) for the updated meat-borne QMRA model.

\begin{tabular}{|c|c|c|c|c|c|}
\hline No. & Heating profile & $\begin{array}{l}\text { Example } \\
\text { product }\end{array}$ & $\begin{array}{l}\text { Minimum } \\
\text { temperature }\end{array}$ & $\begin{array}{l}\text { Most likely } \\
\text { temperature }\end{array}$ & $\begin{array}{l}\text { Maximum } \\
\text { temperature }\end{array}$ \\
\hline 1 & Minced meat & Minced beef & 56.5 & 71.3 & 76.5 \\
\hline 2 & Hamburger & Hamburger & 47.0 & 69.9 & 76.5 \\
\hline 3 & Beef steak & Beef steak & 47.0 & 61.8 & 76.5 \\
\hline 4 & Steak tartare & Beef tartare & 56.5 & 59.5 & 76.5 \\
\hline 5 & $\begin{array}{l}\text { Pan fried } \\
\text { sausage }\end{array}$ & Sausage & 56.5 & 70.1 & 76.5 \\
\hline 6 & $\begin{array}{l}\text { Beef/veal/lamb } \\
\text { large }\end{array}$ & $\begin{array}{l}\text { Leg of } \\
\text { mutton }\end{array}$ & 47.0 & 63.1 & 76.5 \\
\hline 7 & $\begin{array}{l}\text { Beef/veal/lamb } \\
\text { cut }\end{array}$ & Lamb chop & 56.5 & 69.0 & 76.5 \\
\hline 8 & Pork large & $\begin{array}{l}\text { Pork } \\
\text { tenderloin }\end{array}$ & 56.5 & 64.0 & 76.5 \\
\hline 9 & $\begin{array}{l}\text { Ready-To-Eat } \\
\text { meat }\end{array}$ & $\begin{array}{l}\text { Filet } \\
\text { americain }\end{array}$ & 25.0 & 25.0 & 25.0 \\
\hline 10 & $\begin{array}{l}\text { Minced meat } \\
\text { preparation }\end{array}$ & Veal olive & 56.5 & 71.1 & 76.5 \\
\hline
\end{tabular}

\subsection{The probability of infection}

\subsubsection{Probability of infection per portion}

The probability of human infection per infected portion was calculated by using an unscaled exponential dose-response relationship (Guo et al., 2016a) with the number of bradyzoites in the processed infected meat portion as described before (Opsteegh et al., 2011a).

Not all meat portions contain the parasite because not all animals are infected with $T$. gondii. Therefore, the percentages of infected animals were used to calculate the probability that a portion is infected. Lacking an alternative, seroprevalence was used as an indicator of prevalence of $T$. gondii in pork and small ruminant products, despite the fact that studies showed that detection of antibodies to $T$. gondii is in poor to moderate concordance with the presence of the parasite in pigs, small ruminant and chickens depending on the study analysed (Opsteegh et al., 2016a; Opsteegh et al., 2016b). Biologically, those seropositive animals are expected to have tissue cysts and the poor concordance in pigs and small ruminant is likely due to the small sample size or lack of sensitivity of the direct detection methods used. In cattle and horses, there is no or poor concordance (Opsteegh et al., 2016a; Opsteegh et al., 2016b), for instance, T. gondii DNA was detected in 
seronegative cattle (Opsteegh et al., 2011b). Results suggest that the concentration of tissue cysts is low or are not very persistent in cattle. Therefore, T. gondii prevalence obtained from direct methods was used for beef/veal products. The prevalence for pigs was updated from $0.5 \%$ seroprevalence in pigs (van der Giessen et al., 2007) to more recently obtained seroprevalence data in Dutch slaughter pigs of $2.0 \%$ (Swanenburg et al., 2015). The prevalence for beef was updated from $2.0 \%$ based on PCR (Opsteegh et al., 2011b) to $1.6 \%$ based on mouse bioassay (Opsteegh et al., 2016b). The average prevalence for pork and beef was used for pork/beef mixed products (1.8\%). For sheep, no studies have been performed after the previous QMRA has been published. Therefore, the same seroprevalences have been used for lamb (17.7\%) and mutton (53.2\%) (Opsteegh et al., 2010b; Opsteegh et al., 2011a).

\subsection{Number of predicted new infections}

At the population level, the number of predicted infections per meat product was calculated by multiplying the total number of portions consumed and the risk per portion. Because once infected with $T$. gondii, the host generally remains infected for life and develops life-long immunity against reinfection. Reinfection with another strain can happen but only very few cases have been reported (ElbezRubinstein et al., 2009). Therefore, in this study only primary infections are of interest and seroprevalence of $T$. gondii in general population (26\%) and women at childbearing age (19.5\%) (Hofhuis et al., 2011) were used to correct the number of predicted new infections. The final output of the model was the number of predicted infections per meat product and the relative contribution to the total predicted meat-borne $T$. gondii infections per product in the susceptible Dutch general population and pregnant women.

\subsection{Alternative scenario models}

In the first scenario, the salting regression from the previous model developed by Opsteegh et al. (2011a) was used. This was to evaluate the effect of the new salting regression and to compare the relative contribution of meat products to results from the previous QMRA model.

In the second scenario, the salting regression from our baseline model was used, but the salting method for spiced and smoked raw ham was modified. Dry curing and vacuum curing are both common ways to produce ham products in industry, but the percentages of ham products produced by those methods were not available in literature. Therefore, instead of implementing both methods proportionally, 32 days (van Trigt, 2012) of other salting method (brining, vacuum curing, and injection) was used for spiced and smoked raw ham in the base model and changed to 210 days (Gomez-Samblas et al., 2016) of dry curing in this scenario. 


\subsection{Analysis}

The model was implemented in $\mathrm{R}$ version 3.3.1 ( $\mathrm{R}$ Development Core Team, 2006). In addition to the previous model, a bootstrapping analysis was used by randomly sampling the meat products with replacement to examine the uncertainty of consumption frequency. A sampling weight was assigned to each meat product by using its observed frequency divided by the total number of observations from the consumption survey $(7,638$, from two non-consecutive dietary recalls of 3,819 participants). Subsequently, the consumption survey dataset was resampled 100 times with the assigned sampling weights (bootstrap analysis) to mimic the consumption survey, thus, a total of 100 resampled datasets were produced. For each resampled dataset, the result of the QMRA model was based on 5,000 iterations. The mean value of each run and standard deviation were presented as outputs. All inputs for the model are detailed in supplementary material.

\section{Results}

The Dutch National Food Consumption Survey in 2007-2010 recorded 249 meat products from 3,819 participants. After selection, a total of 83 relevant meat products were included in the model based on the pre-retail table. These included 36 pork, 27 beef, 8 veal, 7 lamb, 3 beef/pork mixed, and 2 mutton products. The full list of the 83 meat products and their observed mean portion size together with standard deviation and consumption frequency are shown in Figure 1.

\subsection{Effect of salting on inactivation of $T$. gondii in meat}

A total of eight eligible studies, which reported the effect of salting on $T$. gondii viability were collected for building the generalized linear mixed effects model (Work, 1968; Navarro et al., 1992; Dubey, 1997; Hill et al., 2004; Pott et al., 2013; Neumayerova et al., 2014; Gomez-Samblas et al., 2016; Herrero et al., 2017). The estimated parameters of the model for the effect of salting on inactivation of $T$. gondii from this study and from the previously built salting model by Opsteegh et al. (2011a) are presented in Table 2. Salt concentration, temperature, duration, use of sodium nitrite, salting method, and interaction between salting method and duration remained in the final model. In the eligible studies, four different salting methods were reported (dry curing, brining, vacuum curing, and injection). Since only limited data points concerning vacuum curing were reported and there were no significant differences between brining, vacuum curing and injection, those three methods were grouped together as "other salting method" in the model. The residual versus predicted plot showed a fairly random pattern, and the conditional coefficient $R^{2}$ was 0.88 , indicating that the model provided a decent fit of the data. 


\section{IMPLEMENTED IN A QUANTITATIVE RISK ASSESSMENT OF
MEAT-BORNE HUMAN INFECTION}

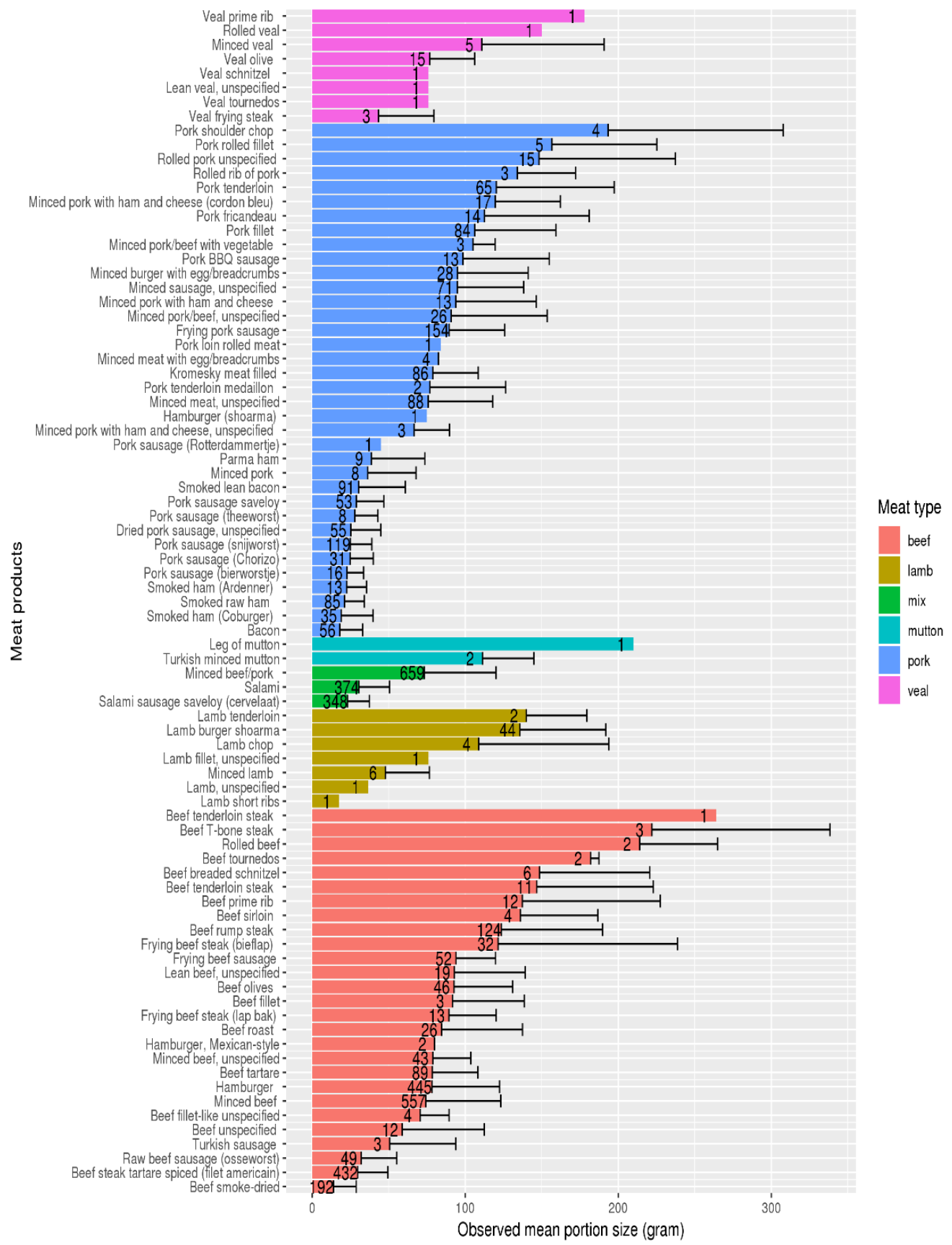

Figure 1. Full list of meat products and the observed mean portion size (bar), standard deviation (error bar), and consumption frequency (number) from Dutch National Food Consumption Survey in 2007-2010. 
Table 2. Estimated parameters for the effect of salting on inactivation of $T$. gondii from a generalized logistic mixed effects regression and a generalized logistic regression (Opsteegh et al., 2011a).

\begin{tabular}{|c|c|c|c|c|}
\hline \multicolumn{4}{|c|}{ Generalized logistic mixed effects regression } & \multirow{2}{*}{$\begin{array}{l}\text { Generalized } \\
\text { logistic regression } \\
\text { (Opsteegh et al., } \\
\text { 2011a) } \\
\text { Estimated } \\
\text { coefficient (SD) }\end{array}$} \\
\hline Variable name & & $\begin{array}{l}\text { Estimated } \\
\text { coefficient } \\
\text { (SD) }\end{array}$ & $p$-value & \\
\hline Constant & & $11.4(2.6)$ & $<0.001$ & $22.3(4.0)$ \\
\hline $\begin{array}{l}\mathrm{NaCl} \\
\text { concentration } \\
(\% \mathrm{w} / \mathrm{v})\end{array}$ & & $-2.6(0.4)$ & $<0.001$ & $-3.3(0.5)$ \\
\hline $\begin{array}{l}\text { Temperature } \\
(\mathrm{C})\end{array}$ & & $-0.6(0.1)$ & $<0.001$ & $-0.4(0.1)$ \\
\hline Duration (day) & & $-0.02(0.0)$ & $<0.001$ & $-0.2(0.1)$ \\
\hline $\begin{array}{l}\text { Temperature } x \\
\text { duration }\end{array}$ & & NA & & $-0.02(0.0)$ \\
\hline $\begin{array}{l}\text { Use of sodium } \\
\text { nitrite (yes/no) }\end{array}$ & No & Reference & & NA \\
\hline & Yes & $-2.0(0.5)$ & $<0.001$ & \\
\hline Salting methods & $\begin{array}{l}\text { Dry curing } \\
\text { Other } \\
\text { salting } \\
\text { methods }\end{array}$ & $\begin{array}{l}\text { Reference } \\
-0.7(2.6)\end{array}$ & 0.8 & NA \\
\hline $\begin{array}{l}\text { Duration } \times \\
\text { salting methods }\end{array}$ & $\begin{array}{l}\text { Duration } \times \\
\text { dry curing } \\
\text { Duration } \times \\
\text { other } \\
\text { salting } \\
\text { methods }\end{array}$ & $\begin{array}{l}\text { Reference } \\
-0.2(0.0)\end{array}$ & $\begin{array}{l}<0.001 \\
<0.001\end{array}$ & NA \\
\hline
\end{tabular}

NA: not applicable 


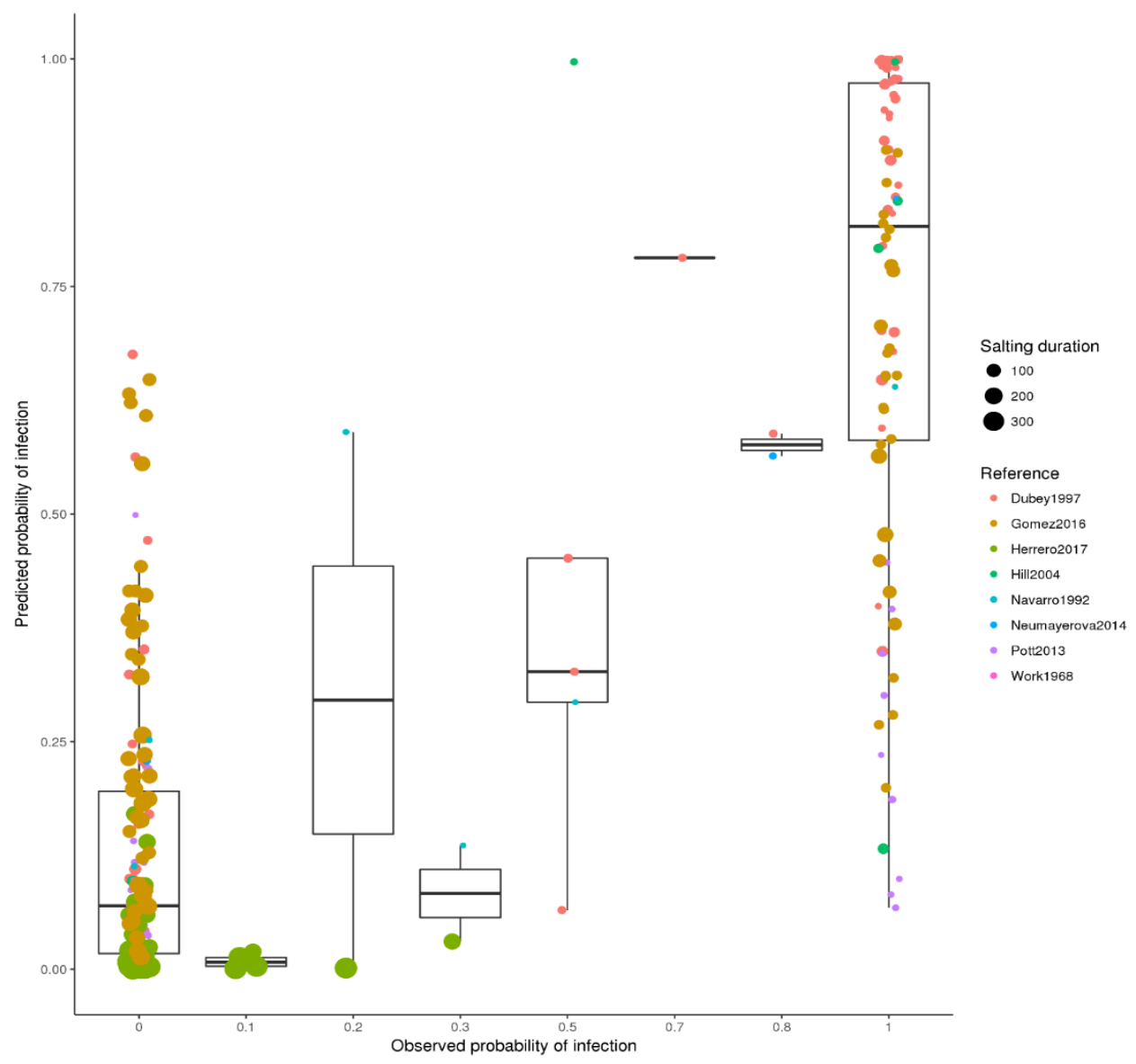

Figure 2. Observed probability of infection (from literature) and predicted probability of infection from the generalized logistic mixed effects regression from salt-treated $T$. gondii infected meat.

Figure 2 shows the observed probability of infection from bioassays and the predicted probability of infection from the fitted mixed effects model. As shown in the figure, the majority of the probabilities of infection from observed data were either zero or one, and the predicted probabilities of infection have a wider range than the observed. This is mainly because usually only one bioassay animal was used for testing the infectivity of salt-treated meat in the studies, but the generalized mixed model has the ability to predict any value between zero and one. In the QMRA model, 28 out of 36 pork, 11 out of 27 beef, 2 out of 8 veal, 2 out of 3 beef/pork mixed products, and 1 out of 2 mutton products were processed with different salt treatments (details in supplementary material). For these products, the effect of salting was calculated by using the new generalized linear mixed regression. 


\subsection{Base model}

At the species level, beef products (84\%) contributed the most to the total number of predicted new infections in the Dutch population, followed by pork (12\%), mutton $(3.7 \%)$, lamb $(0.2 \%)$, pork/beef mixed products $(0.1 \%)$ and veal $(0.01 \%)$ (Table 3). The mean of the total predicted meat-borne infections per year in the general population $(17,081,507)$ (CBS, 2018b) and in pregnant women $(169,836$ live births) (CBS, 2018a) in the base model were 147,765 and 1,214, respectively. At product level, the relative contribution from filet americain $(80 \%$, standard deviation (SD): 1.6), spiced and smoked pork sausage (theeworst in Dutch) (10\%, SD: 0.2 ) and leg of mutton (3.7\%, SD: 1.9$)$ were the highest among the total 83 products (Table 4). Among them, leg of mutton had the highest risk of infection per portion (0.01). For these three products, the values of their input parameters and model outputs from this study and from Opsteegh et al. (2011a) are detailed in Table 4. Ten products with the highest relative contribution to the total number of predicted new infections and their standard deviations based on 100 times simulations for the Netherlands are shown in Figure 3. Complete data on the values for the input variables (consumption data, heating profile, salting parameters, freezing parameters, and Dutch/English translated names of the products), the risk per portion, the relative contribution, and mean number of predicted infections are available for all 83 meat products in the supplementary material.

\subsection{Alternative scenarios}

In our first alternative scenario the salting regression model was set the same as in the previous study from Opsteegh et al. (2011a). Here, beef products remained the most important sources for $T$. gondii meat-borne infection in the Netherlands and they accounted for $67 \%$ of the total predicted infections, followed by pork (31\%), mutton $(2.4 \%)$, beef /pork mixed products $(0.2 \%)$, lamb $(0.1 \%)$ and veal $(0.01 \%)$ (Table 3). The mean of the total predicted meat-borne $T$. gondii infections increased from 147,765 to 228,815 in the general population and from 1,214 to 1,879 in the pregnant women (Table 3 ). At product level, the top three products associated with the highest number of predicted infections were filet americain (59\%, SD: 0.8 ), spiced and smoked pork sausage (12\%, SD: 0.2 ) and spiced and smoked raw ham (7.4\%, SD: 0.1 ). Leg of mutton is not salted and remained the most risky meat product with a risk per portion of 0.01 (Table 4).

In the second alternative scenario, the salting method for spiced and smoked raw ham was modified from 32 days of "other method" (in this case vacuum curing) to "dry curing" for 210 days. This increased the mean predicted number of infections for this product from 4 to 1,204 in the general population and 0.03 to 10 in 
pregnant women. The relative contribution of different meat types changed only slightly compared to the results from the base model: $83 \%, 13 \%, 3.7 \%, 0.2 \%, 0.1 \%$ and $0.01 \%$ for beef, pork, mutton, lamb, beef/pork mixed and veal products respectively. However, spiced and smoked raw ham accounted for $0.8 \%$ of the total predicted infections when processed by dry curing for 210 days and the risk per portion was $3.3 \times 10^{-4}$. This is high in comparison to the results from the base model, in which using other salting method (brining, vacuum curing, and injection) for 32 days resulted in a relative contribution of $0.002 \%$, and a risk per portion of $6.9 \times 10^{-8}$.

Table 3. The mean of the predicted number of meat-borne $T$. gondii infections per livestock species, standard deviation, and their mean relative contribution in the Dutch general population and pregnant women, based on 100 simulations.

\begin{tabular}{lllll}
\hline Model & Species & $\begin{array}{l}\text { Mean of the } \\
\text { predicted } \\
\text { infections/year in } \\
\text { general }\end{array}$ & $\begin{array}{l}\text { Mean of the } \\
\text { predicted } \\
\text { infections/year }\end{array}$ & $\begin{array}{l}\text { Relative } \\
\text { contribution } \\
\text { in } \%)\end{array}$ \\
& & $\begin{array}{l}\text { in pregnant } \\
\text { women (SD) }\end{array}$ & \\
\hline Base model & Beef & $122,941(197)$ & $1,010(2)$ & 84 \\
& Pork & $17,513(120)$ & $144(1)$ & 12 \\
& Mixed & $139(3)$ & $1(0.0)$ & 0.1 \\
& Lamb & $245(19)$ & $2(0.2)$ & 0.2 \\
& Mutton & $6,902(2,798)$ & $57(23)$ & 3.7 \\
& Veal & $24(7)$ & $0(0.05)$ & 0.01 \\
Salting & Total & 147,765 & 1,214 & 100 \\
model & Beef & $151,497(228)$ & $1,244(2)$ & 67 \\
& Pork & $69,657(336)$ & $572(3)$ & 31 \\
& Mixed & $475(3)$ & $4(0.0)$ & 0.2 \\
& Lamb & $268(21)$ & $2(0.2)$ & 0.1 \\
& Mutton & $6,903(2,798)$ & $57(23)$ & 2.4 \\
& Veal & $25(7)$ & $0(0.1)$ & 0.01 \\
& Total & 228,815 & 1,879 & 100 \\
\hline Scenario- & Beef & $122,941(197)$ & $1,010(2)$ & 83 \\
& Pork & $18,713(120)$ & $154(1)$ & 13 \\
& Mixed & $139(3)$ & $1(0.0)$ & 0.1 \\
& Lamb & $245(19)$ & $2(0.2)$ & 0.2 \\
& Mutton & $6,902(2,798)$ & $57(23)$ & 3.7 \\
& Veal & $24(7)$ & $0.2(0.1)$ & 0.01 \\
& Total & 148,965 & 1,223 & 100 \\
\hline
\end{tabular}




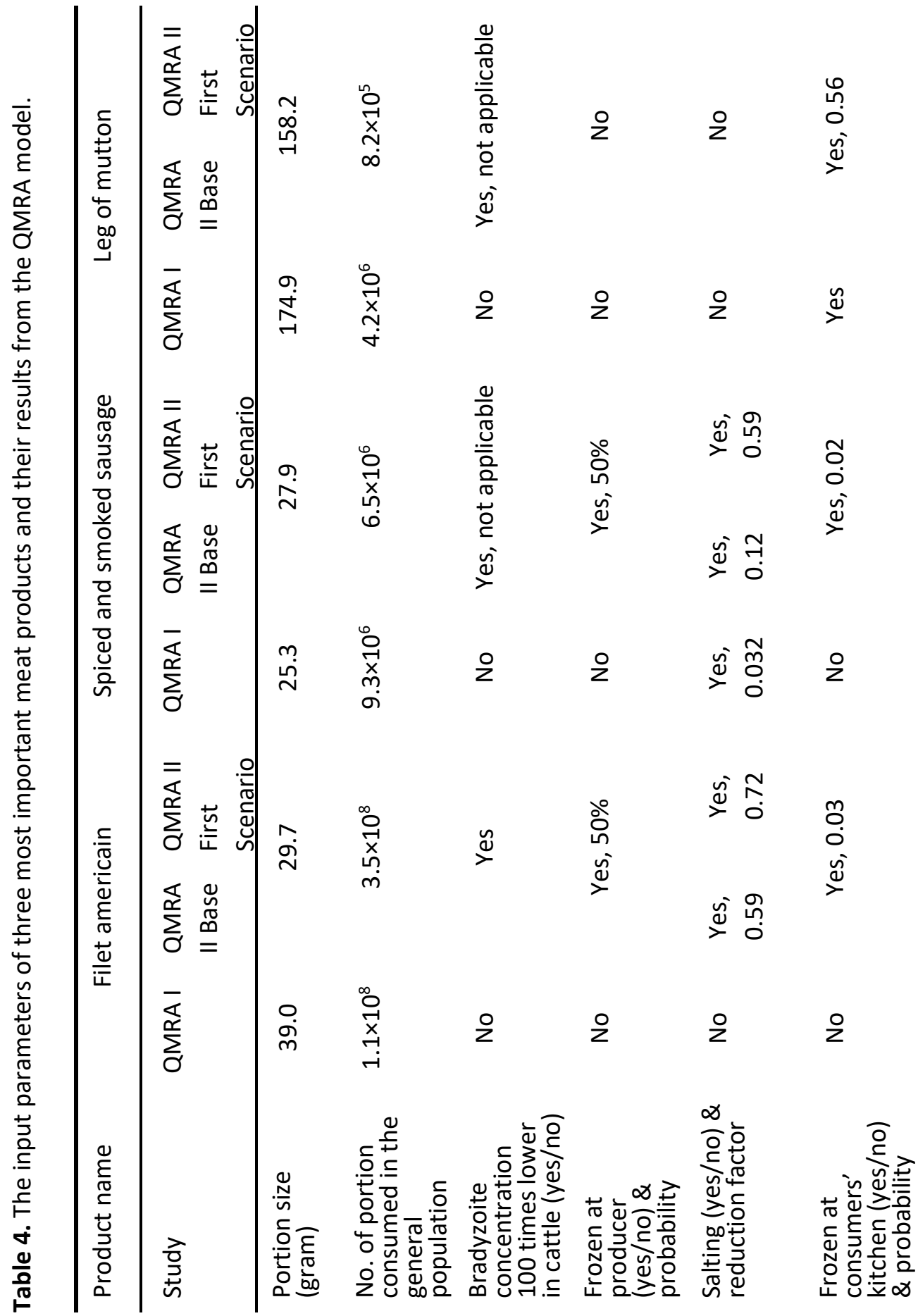
Inputs for model parameters 


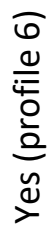

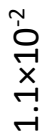

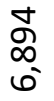

ก

$\stackrel{+}{\sim}$

กั

๖

ก

ก.

$\stackrel{\Perp}{\check{2}}$

à

$\stackrel{m}{\stackrel{n}{N}}$

๑ุ

임

mo
$\stackrel{1}{x}$
vi
vi

ํㅗำ

$\stackrel{\text { ำ }}{\sim}$

N

은

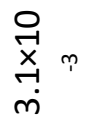

궁

$\stackrel{m}{\sim}$ 위

운

ì

ํํำ

온

กั

ํํำ

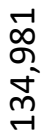

옥

우

은

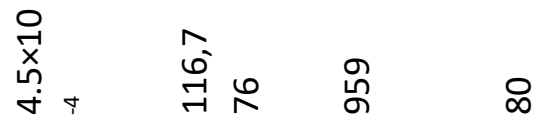

요

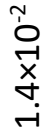

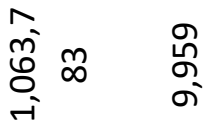

$m$

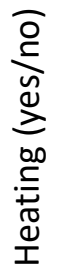

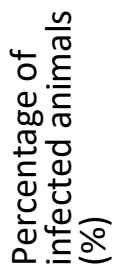

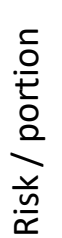

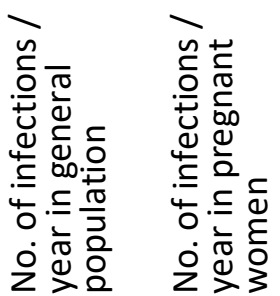

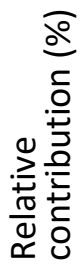

Inputs for model parameters

Model outputs 


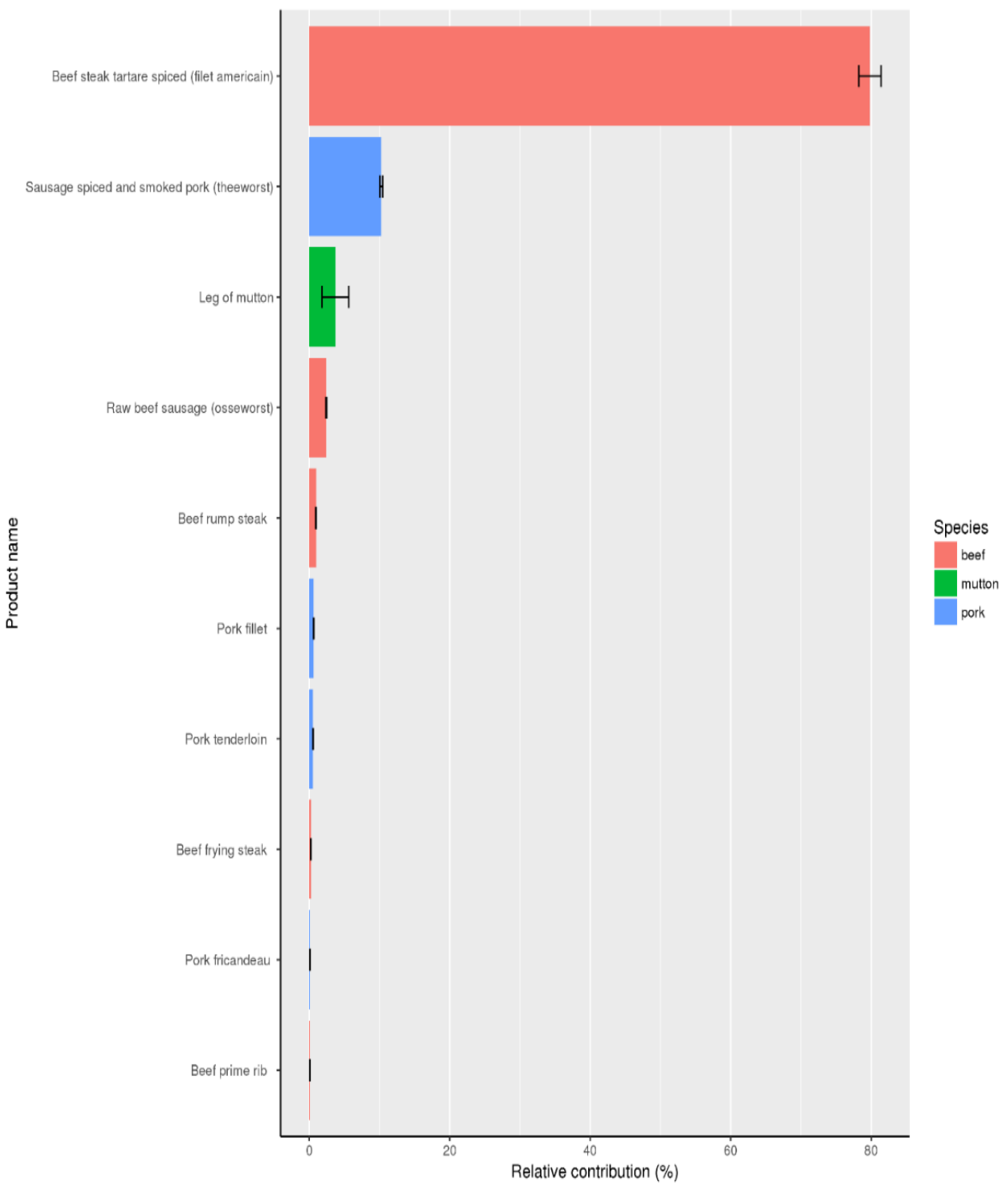

Figure 3. The mean relative contribution to the total number of predicted meatborne $T$. gondii infections for the ten highest ranking meat products, and their standard variations (error bars) based on 100 times simulations. 


\section{Discussion}

In this study, a newly developed salting model together with other up-to-date information were used in a QMRA to calculate the relative contribution and the number of predicted human $T$. gondii infections due to consumption of different meat products in the Netherlands. In addition, two scenario models were conducted to evaluate the impact of the salting inactivation model.

When comparing the relative contributions of different types of meat obtained from this study and the previous study (Opsteegh et al., 2011a), beef remained the most important source of predicted infections with a predicted attribution of $84 \%$ in the current and $68 \%$ in the previous study. The relative contribution of pork products changed slightly (from $11 \%$ to $12 \%$ ), but the predicted number of infections was 18 times lower in this study, although the seroprevalence in pigs was now set 4 times higher than previously. The role of lamb (0.2\%), mutton (3.7\%) and mixed products $(0.1 \%)$ decreased compared to the previous QMRA study. In the new salting regression, more information was collected from literature and was included in the generalized linear mixed regression, such as use of additives and salting method. In the first scenario using the salting regression model published by Opsteegh et al. (2011a), the mean of the total predicted new infections from salted beef products increased from 120,472 in the base model to 149,029 in this scenario. In addition, the mean of the total predicted new infections from salted pork products increased from 15,451 in the base model using the new salting model to 67,585 in this scenario. This means that the new salting model had a stronger effect on $T$. gondii survival. For example, the percentage of inactivated $T$. gondii in infected filet americain after salting was $41 \%$ with the new salting model applied, and it was $28 \%$ when using the regression built based on only one study (Opsteegh et al., 2011a). Thus, the new salting model predicted a lower number of new infections due to consumption of salted products than the previous salting model. Nonetheless, the two most important sources of predicted infections are salted products (filet americain, and spiced and smoked pork sausage). Filet americain (a raw beef spread, similar to "préparé" in Belgium) and spiced and smoked pork sausage ("teewurst" in Germany) are both regional products, they are also popular in neighbouring countries. These two products have a low salting concentration and time, and both lack a heat inactivation step, which is combined with a very high consumption frequency in case of filet americain, and a high starting concentration of $T$. gondii in infected pork for spiced and smoked pork sausage. These results show that simply excluding salted products in QMRA-type studies, would lead to an underestimation of the risk to human infection. However, it was difficult to combine those studies together to quantify the effect of salting on the inactivation of $T$. gondii, because of different study designs, salting treatments, and infectivity examinations. It will be helpful if 
future studies could collect processing-effect data more in line with actual procedures used in industry.

In the second scenario, we replaced the processing parameters of spiced and smoked raw ham to using the dry curing method for 210 days instead of the other salting method (brining, vacuum curing, and injection) for 32 days. This led to a steep increase of the predicted number of new infections as the risk per portion was ten thousand times higher in this scenario $\left(3.3 \times 10^{-4}\right)$ than in the base model $\left(6.9 \times 10^{-8}\right)$. This shows that dry curing is predicted to be less effective than other salting methods for killing $T$. gondii tissue cysts in meat even though the much longer duration of dry curing was taken into account. $T$. gondii survival after dry curing is in line with literature: in Spain, 5\% of 475 commercial Serrano ham samples were found infectious in the mice bioassay (Gomez-Samblas et al., 2015). Viable parasites were found in 3 of 21 (14\%) and 1 of 17 (6\%) from nine months and twelve months dry-cured hams separately (average salt concentration $3 \%$ ) by using bioassay (Herrero et al., 2017). However, none of the mice inoculated with Parma ham which was dry-cured for at least 12 months (average salt concentration 5\%) became infected (Genchi et al., 2017). Results from this scenario show that, in addition to salting inactivation studies in line with industrial procedures, also specific information on the different salting procedures (e.g., method and duration) used to prepare a product is needed for the products to better estimate the survival of $T$. gondii.

Part of the reduction of predicted infections between this study and the previous QMRA study (Opsteegh et al., 2011a) can be explained by the new salting model. However, there are additional new data inputs. In the previous study, the concentration of $T$. gondii bradyzoites for all meat products was based on data from naturally infected sheep hearts (Opsteegh et al., 2010a), and it was assumed that bradyzoite concentration was similar in infected animals of other species. In the current study, a lower concentration in cattle was taken into account. In addition, meat consumption data were obtained from the Dutch National Food Consumption Survey from 2007-2010, instead of from 1998. This means the type of meat products, the volume consumed and the frequency of consumption events were different. In the previous study (Opsteegh et al., 2011a), a total of 50 meat products were included in the model and $40 \%, 34 \%, 25 \%$ and $0.8 \%$ of the selected products were of pork, beef, beef/pork mixed, and sheep origin respectively. While in the present study, based on the new consumption survey data, more meat products were selected (83), and the proportion of beef/pork mixed products (4\%) among all meat products decreased and the proportion of lamb/mutton products increased (11\%) in the survey. Using filet americain as an example, the mean portion size decreased from 39 in 1998 survey to 29 grams, however, the 
frequency of eating tripled from $1.1 \times 10^{8}$ portions to $3.5 \times 10^{8}$ portions (Table 4). Another important product was leg of mutton, because of the highest predicted risk per portion. The observed consumption volume for this product was 210 grams, however, it was only recorded once in the new consumption survey. In order to take the uncertainty of consumption frequency into account, a bootstrapping analysis was included in the calculation by resampling products with different weights from the food consumption dataset. As shown in Figure 3 , and as expected, leg of mutton had the widest variation (i.e., highest uncertainty) of the predicted infections. Moreover, the use of frozen meat for the production of specific products was taken into account by adding a new option of "frozen" at the producer in the model chain. In addition, heating profiles that fit better to the specific products and Dutch situation were used in the calculation. As differences in food processing/preparation, consumption habits and contamination levels in animals influence the role of different types of meat and their relative contribution, our results are not directly applicable to other countries. Therefore, QMRA model should be repeated using country specific input data.

When multiplying the total predicted number of new infections in Dutch pregnant women from the base model with the transmission rate from mother to child $(29 \%)$ (Dunn et al., 1999), this results in a predicted 352 congenital infections per year. In the Netherlands, the estimated number of congenital infections based on newborn screening was 388 per year (Kortbeek et al., 2009), and according to results from Cook et al. (2000) $30 \%$ - 63\% of the seroconversions in pregnant women from six large European cities were due to consumption of meat. This would mean that between 116 and 244 of the 388 congenital infections in the Netherlands are due to meat-borne infections in pregnant women each year. Taking the 30 to $63 \%$ attribution to meat into account, the predicted number of congenital infections from the base model was 1.4 - 3 times higher than from epidemiological data. In contrast, the predicted number of congenital cases obtained from the previous QMRA study was more than 20 times higher than the epidemiological result (Opsteegh et al., 2011a), indicating that our base model better reflects the epidemiological findings (Kortbeek et al., 2009). However, no pregnant women were included in the food consumption survey, thus consumption data from women of childbearing age were used in the model. It is worth noting that productspecific consumption frequencies and volumes, but especially preparation habits, should be more risk-averse during pregnancy. This may have led to an overestimation of the risk for pregnant women. It will be useful if future QMRA studies could focus on specific risk groups when data become available. In this study, beef is still predicted to be the main source of human infection from our QMRA study, whereas a risk factor study in the Netherlands pointed towards pork (Hofhuis et al., 2011). An important role of beef does also fit with a more recent 
meta-analysis of risk factor studies (Belluco et al., 2018). Currently, a prospective case-control study is ongoing in the Netherlands, which will allow comparison of the QMRA to more up-to-date epidemiological data .

The sources of uncertainties and limitations of the QMRA model have been discussed in the previous meat-borne T. gondii QMRA studies (Opsteegh et al., 2011a; Crotta et al., 2017). Important improvements are included in the present study: first, in this study, we took freezing effect at the domestic producers into account in addition to freezing by the consumers. However, the proportion of imported frozen meat in the Netherlands was not included in the calculation due to a lack of product-specific data. Therefore, an underestimation of the fraction of freezing might still be a source of overestimation for the predicted number of infections from our QMRA model. Moreover, as bradyzoites are contained in tissue cysts, which are not homogeneously distributed in the animal, using the prevalence on animal level as indicator of the number of infected portions in our study might be another source of overestimation. In addition, the bradyzoite concentration was based on sheep hearts and it is known that the concentration of parasites also varies between different tissues and the heart is considered a predilection site (Opsteegh et al., 2016a; Opsteegh et al., 2016b). To improve the QMRA further, data on the anatomical distribution between tissues for the different livestock species need to analysed and used as input in the QMRA model. Another source of uncertainty is the dose-response parameter, which was estimated based on mouse experiments. In line with our previous QMRA model (Opsteegh et al., 2011b), Guo et al. (2017) reported a 60 times overestimation when using the unscaled dose-response parameter. This difference between the predicted infections in the QMRA model and the number of infections based on epidemiological data was used to scale the dose-response parameter (Guo et al., 2017). Although the dose-response parameter is one of the most influential parameters with a large uncertainty, there are also other sources of uncertainty in the QMRA model that may have contributed to high model output. With the current updates (most importantly a lower bradyzoite concentration in cattle and a new salting model) our output is already much more in line with the estimates based on epidemiological data without switching to a scaled dose-response parameter.

In conclusion, it is important that the effects of salting and other processing methods are evaluated in line with industrial processing and incorporated in quantitative risk assessment models for meat-borne toxoplasmosis. Our QMRA model using new input data and a stronger effect of salting predicted that beef products remain the most important source of meat-borne $T$. gondii infections in the Dutch population. Filet americain, spiced and smoked pork sausage and leg of 
mutton were the top three products associated with the highest number of predicted infections and risk per portion. This information can guide the development of effective intervention strategies to reduce the disease burden of T. gondii infections.

\section{Acknowledgements}

This study was financed by the Ministry of Health, Welfare and Sports in the Netherlands, and the Netherlands Food and Consumer Product Safety Authority. We thank Eric Evers from Centre for Infectious Disease Control - Zoonoses and Environmental Microbiology, RIVM for sharing pre-retail table, heating and salting datasets of meat products, Marja Beukers and Caroline van Rossum from Centre for Nutrition, Prevention and Health Services, RIVM for sharing Dutch National Food Consumption Survey dataset. 


\section{REFERENCES}

Belluco, S., Patuzzi, I., Ricci, A., 2018. Bovine meat versus pork in Toxoplasma gondii transmission in Italy: A quantitative risk assessment model. Int. J. Food Microbiol. 269, 1-11.

Belluco, S., Simonato, G., Mancin, M., Pietrobelli, M., Ricci, A., 2018. Toxoplasma gondii infection and food consumption: A systematic review and meta-analysis of case-controlled studies. Crit. Rev. Food Sci. Nutr. 58:18, 3085-3096.

CBS, 2018a. Birth; key figures. Available at: https://opendata.cbs.nl/statline/\#/CBS/en/dataset/37422ENG/table?ts=1546632963308. (last accessed on 4 January 2019)

CBS, 2018b. Households; size, composition, position in the household, 1 January. Available at: https://opendata.cbs.nl/statline/\#/CBS/en/dataset/82905eng/table?ts=1546632678413. (last accessed on 4 January 2019)

Chardon, J., Swart, A., 2016. Food consumption and handling survey for quantitative microbiological consumer phase risk assessments. J. Food Prot. 79, 1221-1233.

Condoleo, R., Rinaldi, L., Sette, S., Mezher, Z., 2017. Risk assessment of human toxoplasmosis associated with the consumption of pork meat in Italy. Risk Anal. 38, 1202-1222.

Cook, A.J., Gilbert, R.E., Buffolano, W., Zufferey, J., Petersen, E., Jenum, P.A., Foulon, W., Semprini, A.E., Dunn, D.T., 2000. Sources of Toxoplasma infection in pregnant women: European multicentre case-control study. European Research Network on Congenital Toxoplasmosis. BMJ. 321, 142-147.

Crotta, M., Limon, G., Blake, D.P., Guitian, J., 2017. Knowledge gaps in host-parasite interaction preclude accurate assessment of meat-borne exposure to Toxoplasma gondii. Int. J. Food Microbiol. 261, 95-101.

Dubey, J.P., 1997. Survival of Toxoplasma gondii tissue cysts in $0.85-6 \% \mathrm{NaCl}$ solutions at 4-20 C. J. Parasitol. 83, 946-949.

Dubey, J.P., Kotula, A.W., Sharar, A., Andrews, C.D., Lindsay D.S. 1990. Effect of high temperature on infectivity of Toxoplasma gondii tissue cysts in pork. J. Parasitol, 76, 201-204.

DNFCS. 1998. Dutch National Food Consumption Survey. Available at http://www.rivm.nl/vcp_en/.

DNFCS. 2010. Dutch National Food Consumption Survey. Available at http://www.rivm.nl/vcp en/.

Dunn, D., Wallon, M., Peyron, F., Petersen, E., Peckham, C., Gilbert, R., 1999. Mother-to-child transmission of toxoplasmosis: risk estimates for clinical counselling. Lancet. 353, 1829-1833.

Elbez-Rubinstein, A., Ajzenberg, D., Dardé, M.-L., Cohen, R., Dumètre, A., Yera, H., Gondon, E., Janaud, J.-C., Thulliez, P., 2009. Congenital toxoplasmosis and reinfection during pregnancy: case report, strain characterization, experimental model of reinfection, and review. J. Infecti. Dis. 199, 280-285.

Evers, E.G., Pielaat, A., Smid, J.H., van Duijkeren, E., Vennemann, F.B., Wijnands, L.M., Chardon, J.E., 2017. Comparative exposure assessment of ESBL-producing Escherichia coli through meat consumption. PloS One. 12, e0169589.

FDA, 1999. Home cooking temperature interactive database.

Genchi, M., Vismarra, A., Mangia, C., Faccini, S., Vicari, N., Rigamonti, S., Prati, P., Marino, A.M., Kramer, L., Fabbi, M., 2017. Lack of viable parasites in cured 'Parma Ham' (PDO), following experimental Toxoplasma gondii infection of pigs. Food Microbiol. 66, 157-164.

Gomez-Samblas, M., Vilchez, S., Racero, J.C., Fuentes, M.V., Osuna, A., 2015. Quantification and viability assays of Toxoplasma gondii in commercial "Serrano" ham samples using magnetic capture real-time qPCR and bioassay techniques. Food Microbiol. 46, 107-113.

Gomez-Samblas, M., Vilchez, S., Racero, J.C., Fuentes, M.V., Osuna, A., 2016. Toxoplasma gondii detection and viability assays in ham legs and shoulders from experimentally infected pigs. Food Microbiol. 58, 112120.

Guo, M., Buchanan, R.L., Dubey, J.P., Hill, D.E., Lambertini, E., Ying, Y., Gamble, H.R., Jones, J.L., Pradhan, A.K., 2015. Qualitative assessment for Toxoplasma gondii exposure risk associated with meat products in the United States. J. Food Prot. 78, 2207-2219.

Guo, M., Lambertini, E., Buchanan, R.L., Dubey, J.P., Hill, D.E., Gamble, H.R., Jones, J.L., Pradhan, A.K., 2017. Quantifying the risk of human Toxoplasma gondii infection due to consumption of fresh pork in the United States. Food Control. 73, Part B, 1210-1222.

Guo, M., Mishra, A., Buchanan, R.L., Dubey, J.P., Hill, D.E., Gamble, H.R., Jones, J.L., Du, X., Pradhan, A.K., 2016a. Development of dose-response models to predict the relationship for human Toxoplasma gondii infection associated with meat consumption. Risk Anal. 36, 926-938. 
Guo, M., Mishra, A., Buchanan, R.L., Dubey, J.P., Hill, D.E., Gamble, H.R., Pradhan, A.K., 2016b. Quantifying the risk of human Toxoplasma gondii infection due to consumption of domestically produced lamb in the United States. J. Food Prot. 79, 1181-1187.

Havelaar, A.H., Haagsma, J.A., Mangen, M.J., Kemmeren, J.M., Verhoef, L.P., Vijgen, S.M., Wilson, M., Friesema, I.H., Kortbeek, L.M., van Duynhoven, Y.T., van Pelt, W., 2012. Disease burden of foodborne pathogens in the Netherlands, 2009. Int. J. Food Microbiol. 156, 231-238.

Herrero, L., Gracia, M.J., Perez-Arquillue, C., Lazaro, R., Herrera, A., Bayarri, S., 2017. Toxoplasma gondii in raw and dry-cured ham: The influence of the curing process. Food Microbiol. 65, 213-220.

Hill, D.E., Benedetto, S.M., Coss, C., McCrary, J.L., Fournet, V.M., Dubey, J.P., 2006. Effects of time and temperature on the viability of Toxoplasma gondii tissue cysts in enhanced pork loin. J. Food Prot. 69, 1961-1965.

Hill, D.E., Sreekumar, C., Gamble, H.R., Dubey, J.P., 2004. Effect of commonly used enhancement solutions on the viability of Toxoplasma gondii tissue cysts in pork loin. J. Food Prot. 67, 2230-2233.

Hofhuis, A., van Pelt, W., van Duynhoven, Y.T., Nijhuis, C.D., Mollema, L., van der Klis, F.R., Havelaar, A.H., Kortbeek, L.M., 2011. Decreased prevalence and age-specific risk factors for Toxoplasma gondii IgG antibodies in The Netherlands between 1995/1996 and 2006/2007. Epidemiol. Infect. 139, 530-538.

Kortbeek, L.M., Hofhuis, A., Nijhuis, C.D., Havelaar, A.H., 2009. Congenital toxoplasmosis and DALYs in the Netherlands. Mem. Inst. Oswaldo Cruz. 104, 370-373.

Kotula, A.W., Dubey, J.P., Sharar, A.K. Andrews, C.D., Shen, S.K., Lindsay, D.S. 1991. Effect of freezing on infectivity of Toxoplasma gondii tissue cysts in pork. Journal of Food Protection, 54, 687-690.

Mangen, M.J., Bouwknegt, M., Friesema, I.H., Haagsma, J.A., Kortbeek, L.M., Tariq, L., Wilson, M., van Pelt, W., Havelaar, A.H., 2015. Cost-of-illness and disease burden of food-related pathogens in the Netherlands, 2011. Int. J. Food Microbiol. 196, 84-93.

Mangen, M.J., Friesema, I.H.M., Haagsma, J.A., van Pelt, W., 2017. Disease burden of food-related pathogens in the Netherlands, 2016. Available at: https://www.rivm.nl/bibliotheek/rapporten/2017-0097.pdf.

Mie, T., Pointon, A.M., Hamilton, D.R., Kiermeier, A., 2008. A qualitative assessment of Toxoplasma gondii risk in ready-to-eat small goods processing. J. Food Prot. 71, 1442-1452.

Montoya, J.G., Liesenfeld, O., 2004. Toxoplasmosis. Lancet 363, 1965-1976.

Navarro, I.T., Vidotto, O., Giraldi, N., Mitsuka, R., 1992. Resistance of Toxoplasma gondii to sodium chloride and condiments in pork sausage. Bol. Oficina. Sanit. Panam. 112, 138-143.

Neumayerova, H., Jurankova, J., Salakova, A., Gallas, L., Kovarcik, K., Koudela, B., 2014. Survival of experimentally induced Toxoplasma gondii tissue cysts in vacuum packed goat meat and dry fermented goat meat sausages. Food Microbiol. 39, 47-52.

Opsteegh, M., Langelaar, M., Sprong, H., den Hartog, L., De Craeye, S., Bokken, G., Ajzenberg, D., Kijlstra, A., van der Giessen, J., 2010a. Direct detection and genotyping of Toxoplasma gondii in meat samples using magnetic capture and PCR. Int. J. Food Microbiol. 139, 193-201.

Opsteegh, M., Maas, M., Schares, G., Van der Giessen, J., On Behalf of the Consortium. (2016a). Relationship between seroprevalence in the main livestock species and presence of Toxoplasma Gondii in meat (GP/EFSA/BIOHAZ/2013/01). An Extensive Literature Review. Final Report.

Opsteegh, M., Prickaerts, S., Frankena, K., Evers, E.G., 2011a. A quantitative microbial risk assessment for meatborne Toxoplasma gondii infection in The Netherlands. Int. J Food Microbiol. 150, 103-114.

Opsteegh, M., Schares, G., Blagac, R., van der Giessen, J., On Behalf of the Consortium. (2016b). Experimental studies on Toxoplasma gondii in the main livestock species (GP/EFSA/BIOHAZ/2013/01) Final report. EFSA Supporting Publication 2016; 13( 2):EN-995, 161 pp. doi:10.2903/sp.efsa.2016.EN-995

Opsteegh, M., Teunis, P., Mensink, M., Zuchner, L., Titilincu, A., Langelaar, M., van der Giessen, J., $2010 \mathrm{~b}$. Evaluation of ELISA test characteristics and estimation of Toxoplasma gondii seroprevalence in Dutch sheep using mixture models. Prev. Vet. Med. 96, 232-240.

Opsteegh, M., Teunis, P., Zuchner, L., Koets, A., Langelaar, M., van der Giessen, J., 2011b. Low predictive value of seroprevalence of Toxoplasma gondii in cattle for detection of parasite DNA. Int. J. Parasitol. 41, 343354.

Pott, S., Koethe, M., Bangoura, B., Zoller, B., Daugschies, A., Straubinger, R.K., Fehlhaber, K., Ludewig, M., 2013. Effects of $\mathrm{pH}$, sodium chloride, and curing salt on the infectivity of Toxoplasma gondii tissue cysts. J. Food Prot. 76, 1056-1061.

R Development Core Team, 2006. R: A Language and Environment for Statistical Computing. R Foundation for Statistical Computing, Vienna, Austria.

Swanenburg, M., Boender, G.J., Heres, L., Koeijer, A., Wisselink, H.J., 2015. Toxoplasma prevalence in Dutch slaughter pigs in the period 2012-2014. Epidemiology and control of hazards in pork production chain 
- SAFEPORK. One health approach under a concept of farm to fork. lowa State University Digital Press, Porto, Portugal, 69-72.

Tenter, A.M., Heckeroth, A.R., Weiss, L.M., 2000. Toxoplasma gondii: from animals to humans. Int. J. Parasitol. 30, 1217-1258.

van der Giessen, J., Fonville, M., Bouwknegt, M., Langelaar, M., Vollema, A., 2007. Seroprevalence of Trichinella spiralis and Toxoplasma gondii in pigs from different housing systems in The Netherlands. Vet. Parasitol. 148, 371-374.

van Trigt, P., 2012. Charcuterie 2, praktijkhandboek voor professionele worst- en vleeswarenbereiding. Orange House Edam, the Netherlands.

Work, K., 1968. Resistance of Toxoplasma gondii encysted in pork. Acta. Pathol. Microbiol. Scand. 73, 85-92. 


\section{Chapter}

\section{Quantitative risk assessment of meat-borne Toxoplasma gondii infection in the mainland of China}

Microbial Risk Analysis. 2020, 14, 100090

Huifang Deng ${ }^{1}$, Arno Swart ${ }^{1}$, Yongning $\mathrm{Wu}^{2}$, Xiaowei $\mathrm{Li}^{2}$, Jianhua $\mathrm{Li}^{3}$, Mingyuan $\mathrm{Liu}^{3}$, Marieke Opsteegh ${ }^{1}$, Joke W. B. van der Giessen ${ }^{1}$

Authors' affiliations:

${ }^{1}$ Centre for Infectious Disease Control - Zoonoses and Environmental Microbiology, National Institute for Public Health and the Environment, Bilthoven, the Netherlands

${ }^{2}$ Key Laboratory of China Food Safety Risk Assessment, China National Center for Food Safety Risk Assessment, Beijing, P. R. China ${ }^{3}$ Key Laboratory for Zoonoses Research, Ministry of Education, Institute of Zoonoses, Jilin University, Changchun, P. R. China 


\begin{abstract}
Background: Toxoplasma gondii, a protozoan zoonotic parasite, has a worldwide distribution. It can cause severe pathological damage to the fetus in primary infected pregnant women and life-threatening encephalitis in immunocompromised patients. One of the main transmission routes to humans is consumption of raw or undercooked meat containing viable $T$. gondii tissue cysts. In China, the lack of quantitative information on the risk and incidence of infection due to consumption of meat hampers the development of prevention strategies to reduce the burden of toxoplasmosis.
\end{abstract}

Methods: The aim of this study was to set up a quantitative microbial risk assessment (QMRA) model to estimate the relative attribution of different types of meat (i.e., pork, beef, lamb and mutton, chicken and duck) to human T. gondii infections in China. The model was built based on the structure of the first meatborne $T$. gondii infection QMRA model developed in the Netherlands and applied for seven regions of mainland China. Data on the prevalence of $T$. gondii in livestock and consumption were obtained from China, however information on preparation habits was lacking. Consumption data were available as average consumption amounts per day collected over three days. The results calculated from the QMRA model were at least thousand-fold higher compared to the incidence rate obtained from an epidemiological susceptible-infected (SI) type model.

Results: In general, pork and chicken meat contributed more to the estimated number of infections than beef, small ruminant and duck meat. Cooking temperature was identified as one of the parameters that most strongly affected the estimated incidence rate in the QMRA model. Sensitivity analysis showed that the estimated incidence rate from the QMRA model would be comparable to the results obtained from the $\mathrm{SI}$ model if final cooking temperatures are around 1.7 times higher than the temperatures based on a dataset for American consumers. Scenario analysis demonstrated that it is important to know portion size and frequency of consumption in addition to total consumed amounts to calculate incidence of infection.

Conclusions: Due to uncertainties present in the input data and the at least thousand-fold overestimated incidence rates, the results merely give an indication of the relative importance of different types of meat. However, the QMRA model clearly identified the key knowledge gaps for future research and can subsequently support cost-effective decisions to reduce the disease burden of human toxoplasmosis. 


\section{Introduction}

Toxoplasma gondii is an obligate intracellular protozoan causing toxoplasmosis. It has three infectious stages: free tachyzoites in the bloodstream, bradyzoites in tissue cysts and sporozoites in oocysts (Dubey et al., 1998). All warm-blooded vertebrates serve as intermediate hosts of the parasite and members of the Felidae (cat) family serve as the definitive hosts (Dubey, 1998). Within the intermediate hosts, asexual reproductions occurs. In the initial few days of $T$. gondii infection, rapid multiplication of tachyzoites occurs in the hosts. Tachyzoites transform into bradyzoites under the pressure of immune response to form tissue cysts in multiple organs, including muscles (Montoya and Liesenfeld, 2004). Tissue cysts remain in the organs and muscles lifelong in the hosts in a dormant stage (Montoya and Liesenfeld, 2004). Sexual reproduction only occurs in definitive hosts after ingestion of $T$. gondii tissue cysts from an intermediate host or sporulated oocysts in the environment. The infection of definitive hosts leads to oocyst formation and they are excreted in cat feces as unsporulated form in the environment. The process of sporogony occurs a few days (1-3 days) after deposition. Humans acquire postnatal infection through consumption of raw or undercooked meat containing viable $T$. gondii tissue cysts and through ingestion of infectious oocysts in soil, water or contaminated fruits and vegetables (Hill and Dubey, 2002). The global annual disease burden of toxoplasmosis has been estimated at 1.68 million (95\% UI 1.24-2.45 million) disability-adjusted life years (DALYs) and it ranked third among 11 foodborne parasitic diseases (Torgerson et al., 2015). Half (825,000, 95\% UI 561,000-1.26 million) of these DALYs are attributed to acquired foodborne toxoplasmosis (Torgerson et al., 2015). In humans, the main risk groups are immune-compromised individuals and pregnant women. In immune-compromised individuals, $T$. gondii can become reactivated and the infection can be fatal. If primary infection is acquired during pregnancy, the parasite can be vertically transmitted, leading to congenital toxoplasmosis. This may result in abortion, stillbirth, or lifelong disabilities in the unborn child (Weiss and Dubey, 2009). The global incidence (number of new cases during a certain period of time) of congenital toxoplasmosis is 190,100 cases per year (1.5 per 1,000 live births) (Torgerson and Mastroiacovo, 2013). In China, T. gondii infection is wide-spread and the seroprevalence in the general population has increased from $5.2 \%$ in 1988 1992 to $7.9 \%$ in 2001-2004 (Ji et al., 2005). Although the seroprevalence in China is relatively low compared to other countries (Pappas et al., 2009), this also means that the majority of women in reproductive ages are susceptible to primary infection, and thus at risk for congenital toxoplasmosis. Moreover, a high overall seroprevalence in cancer patients (20\%) and an increase in the number of immunocompromised patients with cancer and HIV make this opportunistic parasitic disease an important public health challenge to China (Jiang et al., 2015; Li et al., 2010; Yang et al., 2013) and strategies to reduce the high disease burden need to 
be considered. So far, considering the lack of commercially available vaccines for use in cats, farm animals and humans (Zhang et al., 2015), and controversy about the effectiveness of treatment and screening programmes in pregnant women (Montoya and Liesenfeld, 2004), prevention of infection seems the most important strategy (Opsteegh et al., 2015). To implement preventive measures, more quantitative knowledge about transmission routes and risk factors are needed. Expert elicitations show that food contributed $56 \%$ of human $T$. gondii infection and different types of red meat (e.g., beef, small ruminant meat, and pork) contribute to $50 \%$ to $64 \%$ of meat-borne $T$. gondii infections (Havelaar et al., 2008; Hoffmann et al., 2017). However, data for the efficacy of prevention policies are scarce and rely on knowledge of the relative attribution of different transmission routes to human infections.

Quantitative microbial risk assessment (QMRA), as defined by World Health Organisation (WHO, 2018), estimates the consequences from a planned or actual exposure to infectious microorganisms and comprises of four phases, hazard identification, hazard characterization, exposure assessment and risk (the probability of developing disease over a certain period of time) characterization. Quantitative risk assessments of $T$. gondii in different meat products have been conducted in the Netherlands, United States, Italy and England (Belluco et al., 2018; Condoleo et al., 2017; Crotta et al., 2017; Guo et al., 2017; Guo et al., 2016; Opsteegh et al., 2011a). In October 2011, the China National Center of Food Safety Risk Assessment Institute (CFSA) was established, which is the sole national agency responsible for improving microbiological risk assessment research (Dong et al., 2015). So far, the relative attribution of different types of meat to human $T$. gondii infections in China is unclear. The aim of the present study was set up a quantitative microbial risk assessment model to determine the attribution of pork, beef, lamb and mutton, chicken, and duck to the estimated incidence rate of $T$. gondii infection in one day in the general population and women of childbearing age in mainland China. The QMRA model was used to estimate the incidence rate and compare the relative importance of various livestock species to meat-borne infections for seven regions of mainland China. Sensitivity and scenario analyses were carried out to identify influential parameters in the model. For validation purposes, the incidence rate of $T$. gondii in humans was additionally estimated by a deterministic susceptible-infected (SI) model. The total population was structured with two sub-populations of susceptible (S) and infected individuals (I). The incidence rate based on epidemiological data obtained from the SI model was compared to the incidence rate generated from the QMRA model. 


\section{Methods}

\subsection{Quantitative microbial risk assessment model}

A QMRA model was built according to the structure of the first meat-borne $T$. gondii infection QMRA model developed in the Netherlands (Opsteegh et al., 2011a) with some modifications. The model comprises three parts: (1) the exposure part describing the distribution, and change in the number of viable $T$. gondii bradyzoites in infected meat due to cooking, (2) the dose-response and (3) the risk characterization parts describing the probability of acquiring $T$. gondii infection in humans via consumption of meat (Fig. 1). In contrast to the Dutch QMRA, meat consumption data on specific meat products was not available in our study, but consumption data of meat originating from different livestock species in China were available. Therefore, the incidence rates were calculated for five meat types: beef, pork, chicken, duck, and small ruminant meat. Sheep and goat meat were not identified separately in the consumption data, but both categorised as small ruminant meat.

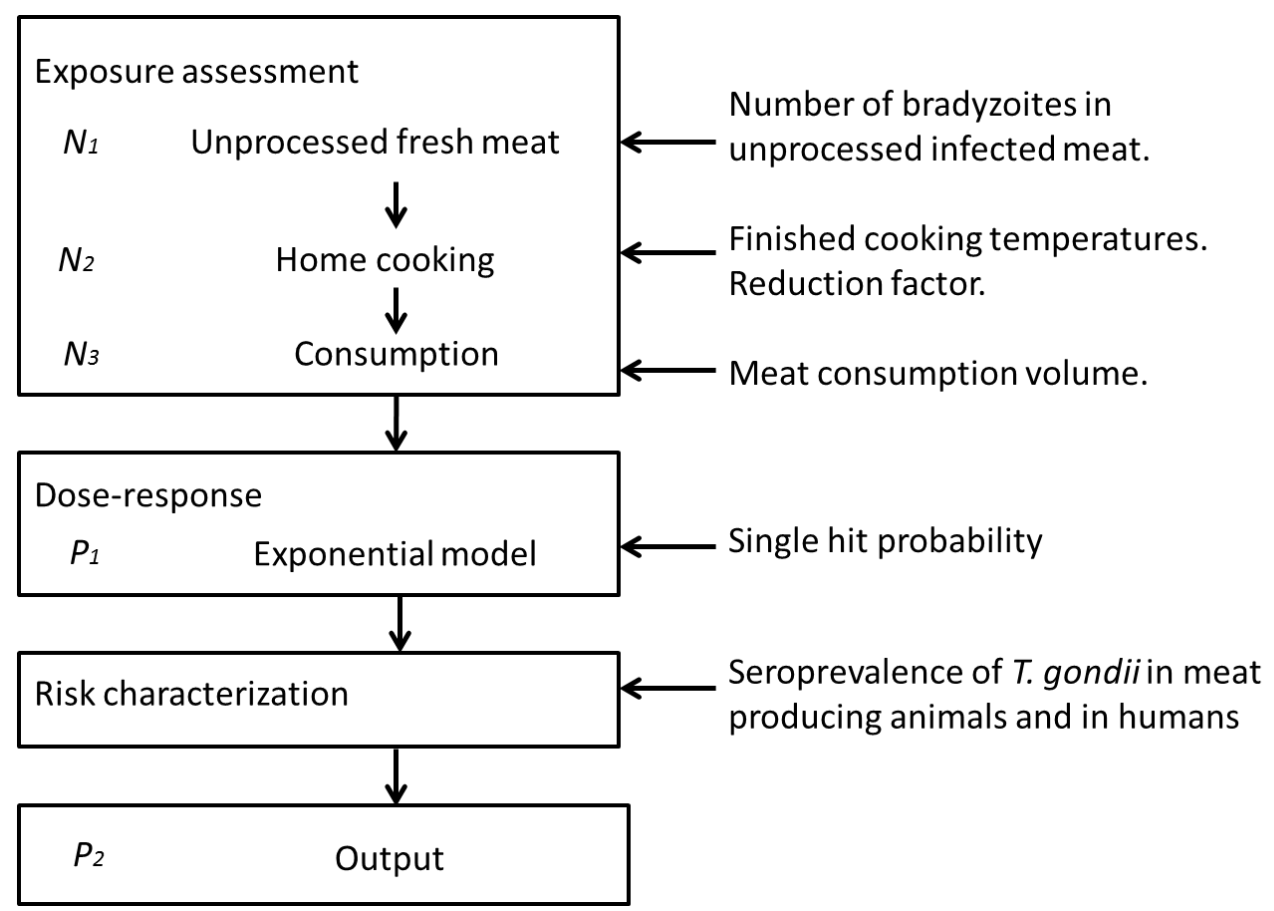

Figure 1. Overview of the risk assessment model used for meat-borne T. gondii infection in the mainland of China. 
For this study, thirty-one provinces of mainland China were grouped into seven regions: central, east, north, north-east, north-west, south and south-west (Fig. 2). For each region, the mean incidence rate of acquiring meat-borne $T$. gondii infection in one day was generated using the QMRA model based on 20,000 iterations.

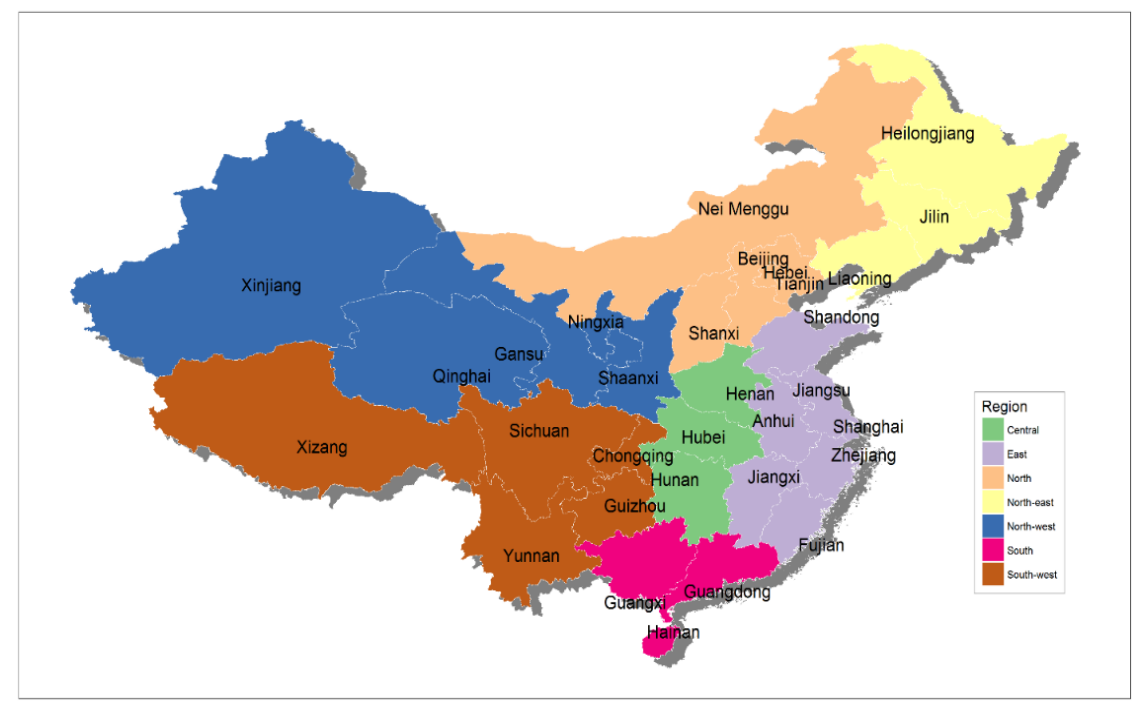

Figure 2. Seven regions of mainland China.

\subsubsection{Exposure assessment}

\subsubsection{Number of bradyzoites in unprocessed infected meat}

As no data were available on the concentration of bradyzoites in naturally infected pigs, cattle and poultry, we used data from naturally infected sheep (Opsteegh et al., 2010). For the $\log _{10}$-transformed concentration of bradyzoites per 100 grams of meat in an infected animal, we used a four-parameter beta distribution with shape parameters 6.5 and 5.7, and boundary parameters 0 and 6.8, as in the Dutch QMRA study (Equation 1 in Table 1) (Opsteegh et al., 2011a). The mean value of the four-parameter beta distribution is 3.6, corresponding to 3,981 bradyzoites per 100 grams of meat.

\subsubsection{Meat consumption amount}

Individual meat consumption data were obtained from the Key Laboratory of China Food Safety Risk Assessment in the Total Diet Study of 2011 (Wu and Li, 2015). The food consumption survey was conducted in different provinces. In each province, one urban location and two rural locations were selected. Participants were asked to report meat consumption amount (gram) at home for a period of 3 days 
(consisting of two week days and one weekend day). In Fujian, Jilin and Qinghai provinces consumption amount for pork rib was reported including bone weight, this was taken into account by multiplying with a fixed factor (0.5) (DondersEngelen, 1997). The reported amount of pig feet collected in Fujian was not included in the total pork consumption amount, because pig feet dishes are always heated to temperatures that inactivate $T$. gondii. Demographic data on age, sex, and location were collected. Average daily consumption of various types of meat was calculated (total consumption amount / 3 days) and used as input in the QMRA model. The amount of imported or domestically produced frozen meat was not taken into account in the model.

Meat consumption data were categorized into seven regions because of the available $T$. gondii seroprevalence data in pregnant women and meat-producing animals from those regions (Deng et al., 2018). Consumption amounts were used to calculate the number of bradyzoites in different types of meat. Meat consumption data were collected as a total daily consumption amount per livestock species, rather than as portion sizes per consumption event in the survey. Portion sizes and frequency of consumption cannot be reliably estimated from three days of data per individual, therefore the incidence rate was calculated based on the average daily consumption amount, which means that the average daily consumption amount is treated as one portion with separate consumption events per livestock species. Therefore, the output of the QMRA is not based on the yearly consumption for the population, but on consumption on a typical day of a crosssection of the population. Variability in the outcome should be interpreted as variation over individuals, not over time. Meat consumption data for pregnant women were not available, thus, consumption data were extracted for women aged between 15-49 years in the dataset by random sampling with replacement of twice the size of the original dataset. Sampling weights were applied for every age and were calculated by using the number of births divided by the number of women at each age in the 2011 yearly statistical book from the National Bureau of Statistics of China (National Bureau of Statistics of China, 2011) (Fig. 3). Data without gender information were excluded from the meat consumption dataset.

The number of bradyzoites per consumed portion in unprocessed infected meat was assumed to follow a Poisson distribution. The number of bradyzoites drawn from the four-parameter beta distribution from previous steps was multiplied with the consumption amount and it was used as rate parameter in the Poisson distribution to obtain integers (Equation 2 in Table 1). The Poisson distribution introduces variability, which also has the desirable effect that small values (e.g., 0.1 ) are not always rounded down, but also have a small probability (about once in 10 times in the 0.1 example) to create a one. 


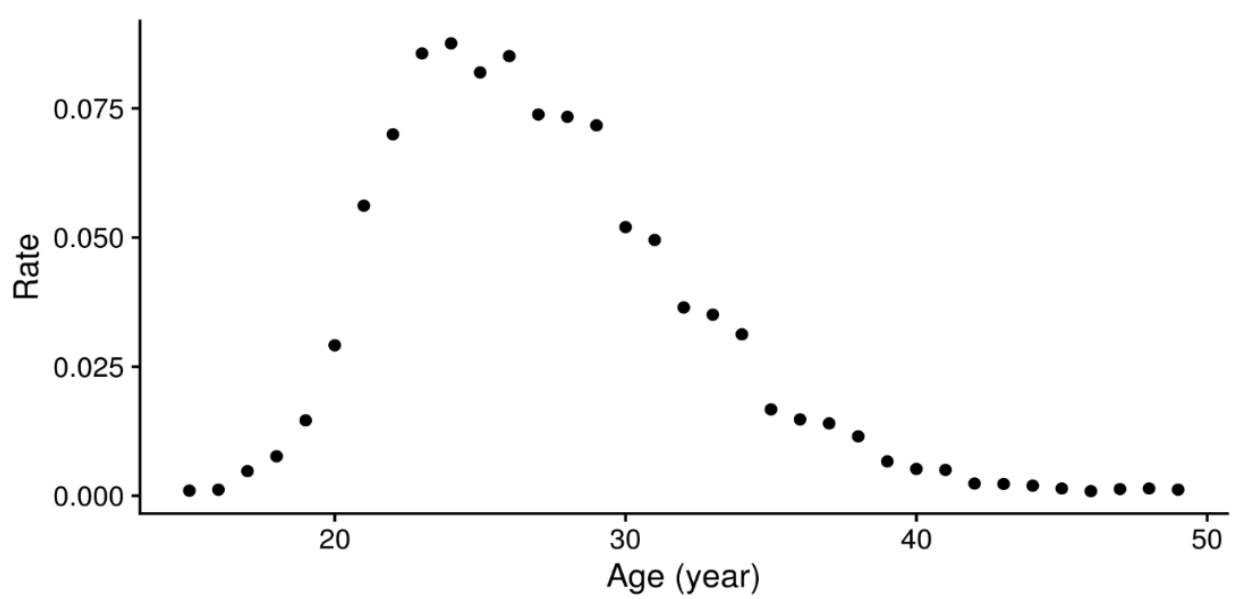

Figure 3. Rate of child birth in women by age in China (based on 2011 yearly statistical book from National Bureau of Statistics of China) used as sampling weights in the QMRA model for meat-borne T. gondii infections in China.

\subsubsection{Number of bradyzoites in infected meat after cooking}

Since no cooking temperature data were available from China, final cooking temperatures recorded by consumers in the USA were used (Ecolab, 2008a). In the dataset, 275,899 and 595 participants recorded the final cooking temperature $\left({ }^{\circ} \mathrm{C}\right)$ for pork, beef and poultry, respectively. Product temperatures were taken immediately upon removal from heat (Ecolab, 2008b). The recorded temperatures ranged from 24 to $138{ }^{\circ} \mathrm{C}$. Laplace distributions were used to fit the data for different types of meat (Fig. 4) as they fitted better than normal distributions (Shapiro-Wilk tests showed that cooking temperature data were not normally distributed, $p<0.001$ ) (supplementary material A Figure 1). The distributions (Equation 7 in Table 1) were used for pork (location parameter $(\mathrm{m})=71.11$, dispersion $(s)=9.88)$, beef $(m=71.11, s=9.82)$, and poultry $(m=75.56, s=9.31)$. As no final cooking temperatures were collected for small ruminants, the same distribution as for beef $(m=71.11, s=9.82)$ was used for small ruminant meat. In the model, temperatures from the Laplace distributions were truncated to be above $25{ }^{\circ} \mathrm{C}$. The number of bradyzoites in infected meat after cooking was calculated by multiplying the number of bradyzoites in infected unprocessed meat from the previous step with a reduction factor (Equation 3 in Table 1). The reduction factor (Equation 4 in Table 1) was calculated based on a mouse bioassay experiment (Dubey et al., 1990), by fitting the data to a logistic regression model (Equation 6 in Table 1), and together with a reversed dose-response relation in mice (Equation 5 in Table 1) as described in detail in the Dutch QMRA model (Opsteegh et al., 2011a). 


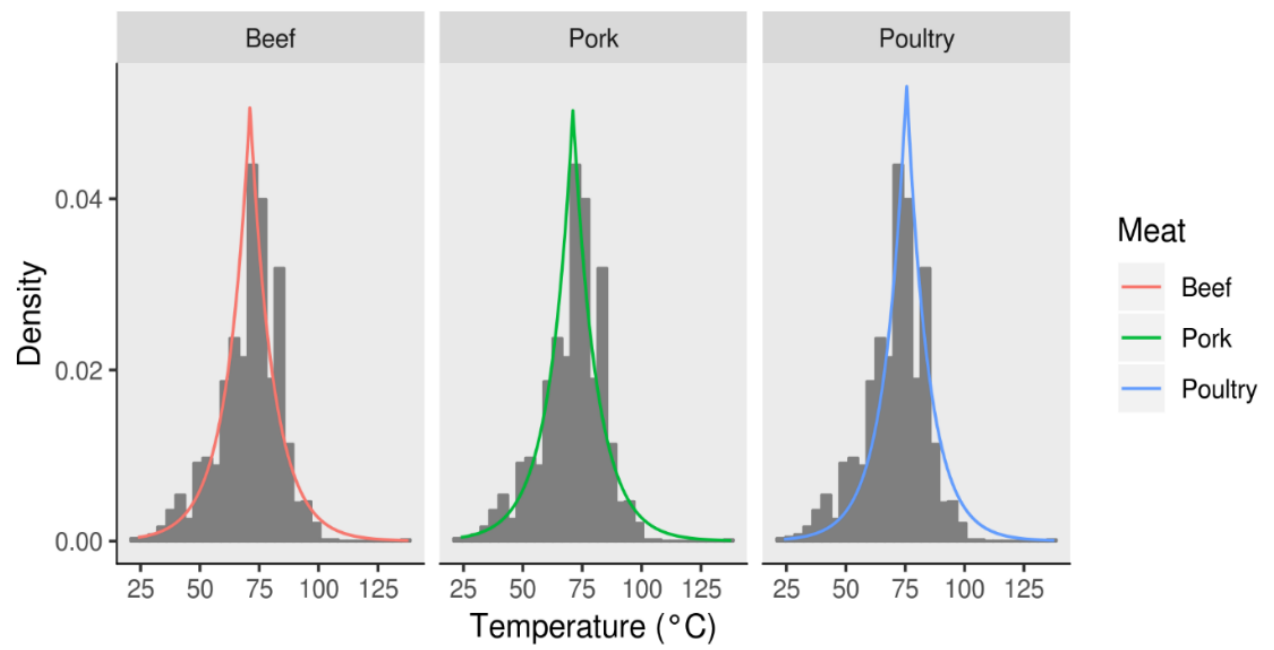

Figure 4. Observed final cooking temperatures from Ecolab (2008a) and fitted distributions used in the QMRA model for meat-borne T. gondii infections in China.

\subsubsection{Dose-response}

The exponential dose-response model (Equation 8 in Table 1) was selected due to its simplicity and convenience to compare with previous study (Opsteegh et al., 2011a). The consequences of $T$. gondii infection involve multiple symptoms, but the infection can also remain asymptomatic in people. Thus, in the dose-response assessment we used infection rather than disease as our endpoint. Since no human dose-response relationship is available, the single-hit probability of infection (the probability for one organism to successfully initiate an infection) from a bradyzoite (type II) was estimated based on mouse experiments $(r=0.001535)$ (Guo et al., 2016). With the number of bradyzoites per consumed amount in the processed meat and the dose-response relationship, the probability of infection through infected meat was calculated.

\subsubsection{Risk characterization}

\subsubsection{Seroprevalence of $T$. gondii in meat producing animals and humans}

Not all animals are infected with $T$. gondii, thus, the probability of human infection was calculated by multiplying the probability of infection through meat with the seroprevalence of $T$. gondii in meat producing animals. The seroprevalence of $T$. gondii in meat producing animals in seven regions of mainland China was obtained from a systematic review using Bayesian hierarchical meta-analysis (Deng et al., 2018). These sensitivity and specificity adjusted seroprevalences were used in the model (supplementary material A, Table 1). Since no information on the seroprevalence in ducks is available, we assumed that the farming systems for 
ducks and chickens were similar, thus the seroprevalence in chickens was used for ducks in our model. By implementing the regional seroprevalence in different livestock species, it is assumed that meat was produced and consumed in the same region.

Once infected with $T$. gondii, the host generally remains infected for life and develops life-long immunity against reinfection from the same genotype. Therefore, only primary infections are of interest and the seroprevalence of $T$. gondii in the Chinese general population (7.9\%) from the latest national survey was used for all regions (Ji et al., 2005). For women of childbearing age, region-specific seroprevalence estimates were obtained by a systematic review of the literature (Deng et al., 2018).

Table 1. Equations used in the meat-borne $T$. gondii infection quantitative microbial risk assessment model.

\begin{tabular}{|c|c|c|c|}
\hline Variable & Equation/distribution/value & Reference & No. \\
\hline \multicolumn{4}{|l|}{ Exposure assessment } \\
\hline $\begin{array}{l}N_{1} \text { : number of } \log _{10^{-}} \\
\text {transformed bradyzoites } \\
\text { in } 100 \text { grams } \\
\text { unprocessed infected } \\
\text { meat }\end{array}$ & $\begin{array}{l}N_{1} \sim \text { Beta general (shape } 1= \\
6.5, \text { shape } 2=5.7, \min =0, \\
\max =6.8)\end{array}$ & $\begin{array}{l}\text { (Opsteegh } \\
\text { et al., } \\
2011 a)\end{array}$ & 1 \\
\hline $\begin{array}{l}\mathrm{N}_{2} \text { : number of } \\
\text { bradyzoites per } \\
\text { consumed amount per } \\
\text { meat type }\end{array}$ & $\begin{array}{l}N_{2} \sim \text { Poisson }\left(\lambda=C_{\text {meat }} \times 10^{N_{1}}\right. \\
/ 100)\end{array}$ & $\begin{array}{l}\text { (Opsteegh } \\
\text { et al., } \\
2011 \mathrm{a} \text { ) } \\
\text { (Wu and Li, }\end{array}$ & 2 \\
\hline $\begin{array}{l}\mathrm{C}_{\text {meat: }} \text { consumed amount } \\
\text { per meat type (gram) }\end{array}$ & $\begin{array}{l}C_{\text {meat }}: \text { China Total Diet Study of } \\
2011\end{array}$ & 2015) & \\
\hline $\begin{array}{l}N_{3} \text { : number of } \\
\text { bradyzoites after home } \\
\text { cooking per consumed } \\
\text { portion }\end{array}$ & $N_{3}=N_{2} \times \operatorname{RF}(\mathrm{T})$ & $\begin{array}{l}\text { (Opsteegh } \\
\text { et al., } \\
\text { 2011a) }\end{array}$ & 3 \\
\hline $\begin{array}{l}\mathrm{RF}(\mathrm{T}) \text { : reduction factor } \\
\text { for temperature }\end{array}$ & $\mathrm{RF}(\mathrm{T})=\frac{D(T)}{D\left(T_{0}\right)}$ & $\begin{array}{l}\text { (Opsteegh } \\
\text { et al., } \\
2011 a)\end{array}$ & 4 \\
\hline $\begin{array}{l}\mathrm{D}(\mathrm{T}) \text { : number of } \\
\text { bradyzoites (dose) }\end{array}$ & $D(T)=-\frac{\ln \left(1-P_{0}(T)\right)}{r_{0}}$ & & 5 \\
\hline $\begin{array}{l}P_{0}(\mathrm{~T}) \text { : probability } \\
\text { infection for mice }\end{array}$ & $\begin{array}{l}P_{0}(T) \\
=1 /\left(1+e^{-(44.181-0.834 \times T)}\right)\end{array}$ & $\begin{array}{l}\text { (Opsteegh } \\
\text { et al., } \\
\text { 2011a) }\end{array}$ & 6 \\
\hline
\end{tabular}


Table 1. (continued)

\begin{tabular}{|c|c|c|c|}
\hline Variable & Equation/distribution/value & Reference & No. \\
\hline $\begin{array}{l}r_{0}: \text { probability of a single } \\
\text { bradyzoite initiating } T . \\
\text { gondii infection in mice }\end{array}$ & $r_{0}=0.011$ & & \\
\hline \multirow[t]{2}{*}{$\mathrm{T}$ : temperature $\left({ }^{\circ} \mathrm{C}\right)$} & $\begin{array}{l}\mathrm{T} \sim \text { Laplace } \\
\text { (m = location, } \mathrm{s}=\text { dispersion) }\end{array}$ & $\begin{array}{l}\text { (Ecolab, } \\
\text { 2008a) }\end{array}$ & 7 \\
\hline & $\begin{array}{l}\text { Pork: } \mathrm{m}=71.11, \mathrm{~s}=9.88 \\
\text { Beef \& small ruminants: } \\
\mathrm{m}=71.11, \mathrm{~s}=9.82 \\
\text { Poultry: } \mathrm{m}=75.56, \mathrm{~s}=9.31\end{array}$ & & \\
\hline $\begin{array}{l}T_{0} \text { : temperature before } \\
\text { cooking }\left({ }^{\circ} \mathrm{C}\right) \\
\text { Dose-response }\end{array}$ & $T_{0}=25$ & & \\
\hline $\begin{array}{l}P_{1} \text { : probability of human } \\
\text { infection (/meat type in } \\
\text { one day) }\end{array}$ & $P_{1}=1-\mathrm{e}^{\left(-r_{1} \times N_{3}\right)}$ & $\begin{array}{l}\text { (Guo et al., } \\
2016 \text { ) }\end{array}$ & 8 \\
\hline $\begin{array}{l}r_{1}: \text { probability of a single } \\
\text { bradyzoite initiating } T . \\
\text { gondii infection in } \\
\text { humans } \\
\text { Risk characterization }\end{array}$ & $r_{1}=0.001535$ & & \\
\hline $\begin{array}{l}P_{2}: \text { probability of } \\
\text { infection through } \\
\text { consumption of meat in } \\
\text { human population } \\
\text { (/meat type in one day) }\end{array}$ & $P_{2}=P_{1} \times P_{\text {animal }} \times\left(1-P_{\text {human }}\right)$ & $\begin{array}{l}\text { (Opsteegh } \\
\text { et al., } \\
2011 a)\end{array}$ & 9 \\
\hline $\begin{array}{l}P_{\text {animal }}: \text { adjusted } \\
\text { seroprevalences per }\end{array}$ & $\begin{array}{l}P_{\text {animal }}: \text { supplementary } \\
\text { material A Table } 1\end{array}$ & $\begin{array}{l}\text { (Deng et al., } \\
\text { 2018) }\end{array}$ & \\
\hline animal type in seven & Phuman-prgnant women $:$ & & \\
\hline regions of mainland & supplementary material A & & \\
\hline \multirow[t]{2}{*}{ China } & Table 1 & & \\
\hline & $P_{\text {human-general population: }} 7.9 \%$ & (Ji et al., & \\
\hline $\begin{array}{l}P_{\text {human }} \text { : seroprevalence of } \\
\text { human population }\end{array}$ & & 2005) & \\
\hline
\end{tabular}


The output of the risk characterization part was calculated by using equation 9 in Table 1. The probabilities of infection through eating different types of meat in one day were calculated from this part of the model. The final output from the model is the mean incidence rates of acquiring $T$. gondii infection in one day for the general population and women of childbearing age in seven regions of mainland China.

\subsubsection{Sensitivity analysis}

A local sensitivity analysis was performed on five input parameters (number of bradyzoites in meat, meat consumption amount, cooking temperature, single-hit probability, and animal seroprevalence) which were considered as the most important factors in the model. This approach was used to identify and prioritize the most influential inputs of the model. The effect of these parameter value on the model outcome was assessed by changing the parameter value in both directions. Parameter values were one at a time multiplied by a sequence of values starting from 0.1 to 2.0 with an increment of 0.1 , while all the other input variables were kept the same. From every multiplied value, the mean of $T$. gondii infection in one day per 1,000 persons in seven regions of mainland China through consumption of meat was calculated based on 30 iterations (due to the memory limits of the program). The obtained results were $\log _{10}$-transformed in order to make the relationship between input and output of the sensitivity analysis more clear.

\subsubsection{Scenario analysis}

The first scenario was set to evaluate the effect of heating temperature. Because cooking temperature data were not available from China, data from American consumers was used in the base model. However, the cooking temperatures used in Chinese kitchens are assumed to be higher, thus the cooking temperature distributions were shifted to the right by adding an arbitrary $10{ }^{\circ} \mathrm{C}$ to the location parameters of the Laplace distributions in this scenario.

In order to investigate the effect of assuming that the consumption amount in a day was consumed as one portion with separate consumption events per livestock species, a second scenario with the same total consumption amount as described in the previous baseline model but divided over two consumption events per livestock species was evaluated. The estimated incidence rates from this scenario were compared to the results from baseline model which was built with only one consumption event per livestock species in a day.

A third scenario was set for evaluating the effect of using seroprevalence in cattle due to the lack of concordance between detection of antibodies and the presence 
of T. gondii in cattle (Opsteegh et al., 2011b). The seroprevalence was used for cattle in the base model as regional prevalence of $T$. gondii infection in cattle estimated from direct detection was not available, and only limited work on direct detection of $T$. gondii has been published in Jilin province, China (Ge et al., 2014). In this scenario, a prevalence of $2.6 \%$ based on results from a systematic review of T. gondii in beef estimated by direct methods (Belluco et al., 2016), was used for all regions.

In the fourth scenario, the bradyzoite concentration in cattle was set to 100 times lower than in the other species, because experimental studies showed that the concentration of bradyzoites was at least a hundred times lower in cattle than in sheep (Opsteegh et al., 2011).

The model was implemented in $\mathrm{R}$ version 3.3.1 ( $\mathrm{R}$ Development Core Team, 2006). The outputs were the mean incidence rates based on 20,000 iterations, with this number of iterations the model captures the variability. The uncertainties of the results were taken care of by the scenario analysis. The $R$ code can be found in the supplementary material B.

\subsection{Deterministic susceptible-infected (SI) model}

A deterministic SI model dealing with $T$. gondii transmission dynamics in human population was built to compare with the incidence rate generated from our QMRA model. In general, once infected with $T$. gondii, the host remains infected for life and develops life-long immunity against reinfection for $T$. gondii. Only very few cases of reinfection with another genotype have been reported (ElbezRubinstein et al., 2009), possibly due to consumption of imported raw meat from other countries. In this study, only primary infections were taken into account. We therefore assumed that no reinfection occurs in the model. The total human population $(N)$ is categorized into only two compartments, susceptible $(S)$ and immune (I), with $N=S+I$. A closed population with age involved (a) was used for the model. The dynamics of $T$. gondii infection in humans are described by the following ordinary differential equation (ODE):

$$
\begin{aligned}
& \frac{d S}{d a}=-\lambda S \\
& \frac{d I}{d a}=\lambda S
\end{aligned}
$$

When at age of zero, the number of susceptible equals the total population $(N)$, and the number of persons with immunity (I) equals zero:

$$
S(0)=N
$$




$$
I(0)=0
$$

We get:

$$
\begin{aligned}
& S(a)=N \times \exp (-\lambda \times a) \\
& I(a)=N \times(1-\exp (-\lambda \times a))
\end{aligned}
$$

and

$$
\begin{gathered}
p(a)=\frac{I(a)}{N}=1-\exp (-\lambda \times a) \\
\hat{p}=\frac{1}{A} \int_{0}^{A} p(a) \mathrm{d} a=1-\frac{1}{\lambda \times A} \times(1-\exp (-\lambda \times \mathrm{A}))
\end{gathered}
$$

Where $p$ is the seroprevalence and $\lambda$ is the incidence rate of $T$. gondii infection, $\hat{p}$ is the average seroprevalence of the Chinese population, from age zero to A (not weighted by population age structure).

\section{Results}

\subsection{Meat consumption amount in China}

Individual meat consumption amount was collected from 11,819 participants in 20 provinces of China in 2011 (Wu and Li, 2015). The sample size ranged from 186 to 1,403 participants in different provinces (supplementary material A Table 2). The numbers of participants living in rural and urban areas were 7,664 and 4,155 respectively. Age information was available from 11,706 participants, the number of male and female participants were 5,706 and 6,000. The age ranged from 0 to 97 years.

The ranges of mean consumption amount per day collected over 3 days of pig, cattle, small ruminant, chicken, and duck meat in the seven regions were 70-148, 41-80, 40-183, 85-164, and 42-84 grams, respectively (supplementary material A Figure 3). The frequency, mean and standard deviation of the consumption amount per day per meat type in different provinces were shown in supplementary material A Table 3. In addition, pork (61.5\%-99.0\%) had the highest frequency of consumption among all the other meat types (0.0\%-44.4\%). There was no significant difference in meat consumption amount between urban and rural consumers, except for cattle and small ruminant meat $(p<0.05)$. Small ruminant consumption data were not available in the south of mainland China and duck consumption data was not available in the north-west.

\subsection{Estimated risk of $T$. gondii infection through consumption of different meat}

The mean incidence rates of meat-borne $T$. gondii infection per 1,000 meat consumers in one day in seven regions are shown in Table 2 . The mean number of the estimated $T$. gondii infections per 1,000 meat consumers in one day due to consumption of pork, chicken, small ruminant, duck and cattle ranged respectively 
from 9.3 to17, 3.3-7.9, 6.6-11, 2.5-6.5, and 2.8-5.7 in the general population in seven different regions (Table 2). Random sampling with a sampling weight was conducted on 2,887 women aged 15-49 years old in the consumption dataset to calculate the mean incidence rate for women of childbearing age. The mean number of the estimated $T$. gondii infections per 1,000 female meat consumers of childbearing age in one day due to consumption of pork, chicken, small ruminant, duck and cattle ranged respectively from 9.5 to $18,3.4-8.4,6.3-11,2.4-7.5$, and 3.0-6.0 in the seven regions (Table 2).

The mean incidence rates of meat-borne $T$. gondii infection per 1,000 persons in one day in seven regions of mainland China are shown in Fig. 5 (Base Model). A wide variation of estimated incidence rate was observed in different region. Southwest had the highest estimated incidence rate, while north-east had the lowest. In general, pork and chicken meat contributed more to the estimated infections than beef, small ruminant and duck meat.

\subsection{Sensitivity analysis}

The number of bradyzoites in meat, meat consumption amount, final cooking temperature, animal seroprevalence, and single-hit probability were selected to check their relationship with the estimated incidence rate of meat-borne $T$. gondii infection. From the sensitivity analysis result, final cooking temperature was found to be the most important variable in the model that affects the number of meatborne $T$. gondii infections in the Chinese population (Fig. 6). As expected, the effect of some variables (number of bradyzoites in meat, meat consumption amount, animal seroprevalence, and single-hit probability) was linear, because they were multiplicative in the equations (Table 1). The sensitivity analysis results of these variables are shown in supplementary material A Figure 2.

\subsection{Scenario analysis}

In the first scenario when increasing the cooking temperature, the mean number of estimated infections is shown in Fig. 5 Scenario A. Compared to the base model, the mean number of estimated infections due to consumption of different types of meat all decreased around $60 \%$. But the relative attribution of different meat remained the same as in the base model.

The relative attribution of estimated $T$. gondii infections due to consumption of different meat from the scenario when the daily consumption amount was divided over two portions is shown in Fig. 5 Scenario B. For all types of meat, the estimated number of cases almost equally increased compared to the results from the base model. However, pork and chicken meat still contributed more to the estimated number of infections than other meat types. 


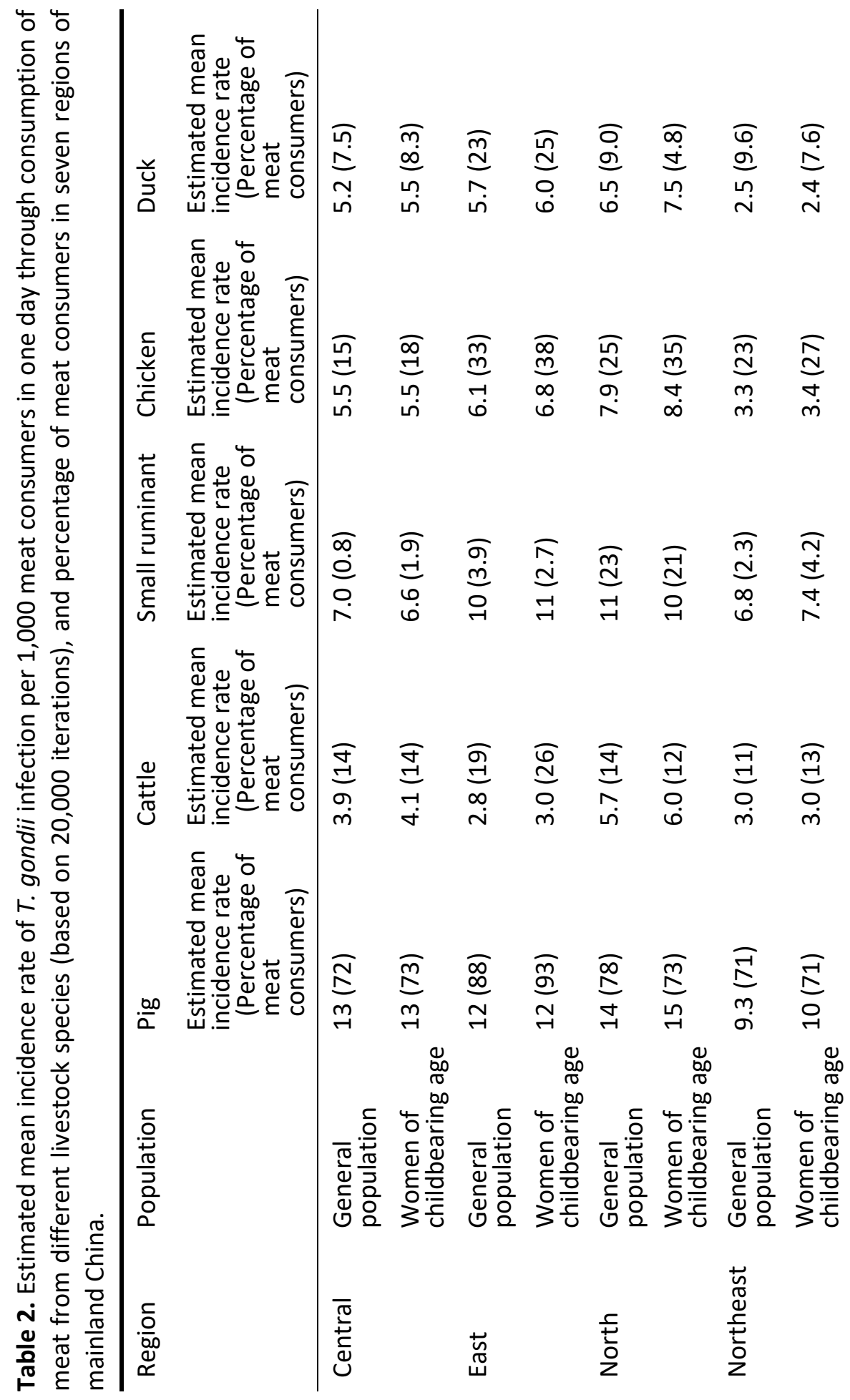




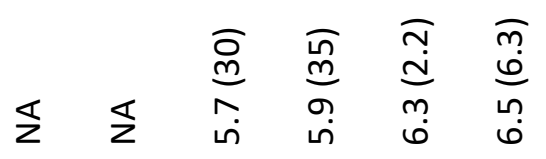

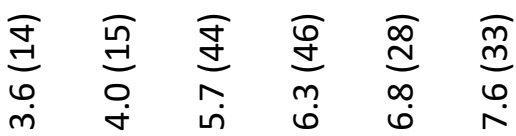

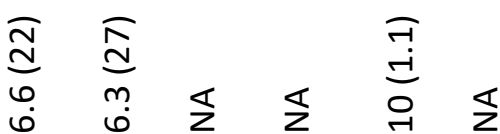

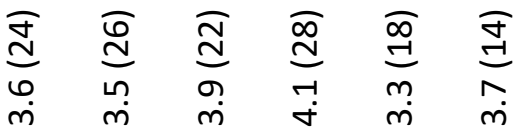

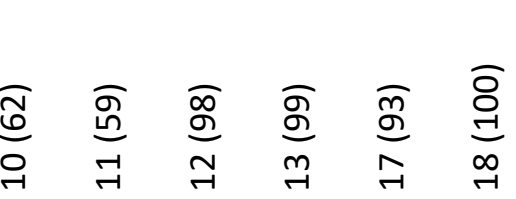

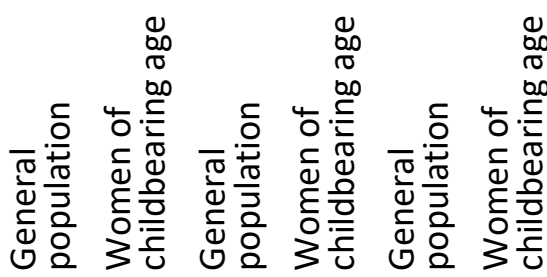

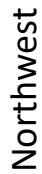

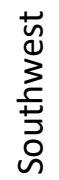

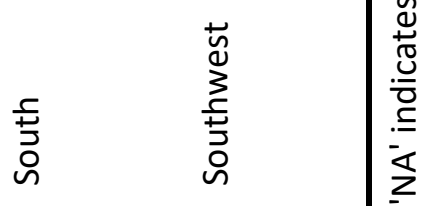


In the third scenario when changing the prevalence of $T$. gondii in cattle to $2.6 \%$ for all regions, the estimated infections due to consumption of beef reduced in the general population and in women of childbearing age (Fig. 5 Scenario C). Beef became less important for meat-borne $T$. gondii infection in mainland of China than other types of meat.

In the fourth scenario, after setting the concentration of bradyzoites 100 times lower in cattle, the relative attribution of different types of meat to the estimated number of cases remained almost the same compared to base model. However, the estimated infections due to consumption of beef was 10 times lower than the results from the base model. Thus, the attribution of beef was further reduced (Fig. 5 Scenario D).

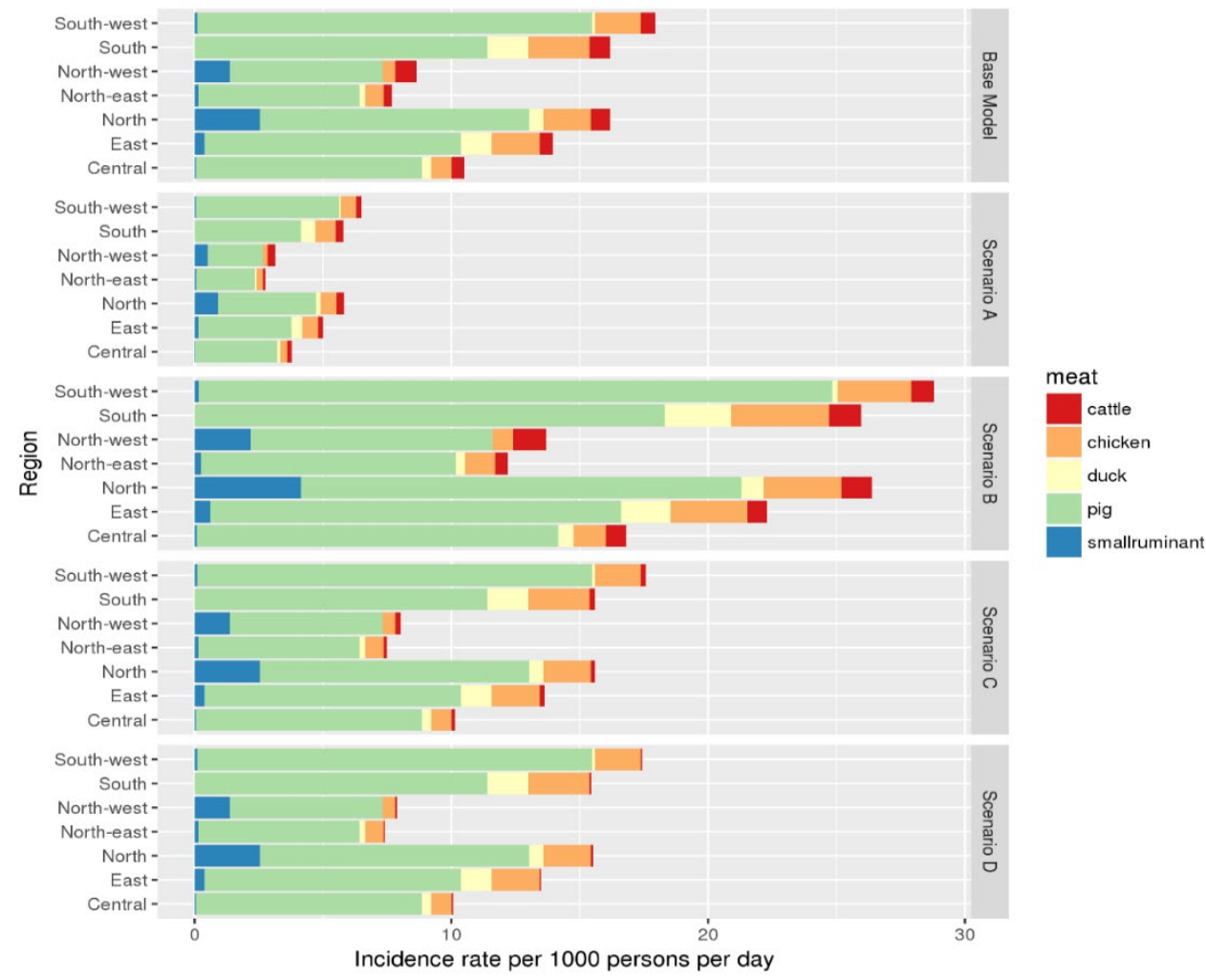

Figure 5. The attribution of different livestock species to the total number of estimated $T$. gondii infections from general population in seven regions of China, obtained from the base model, scenario A (cooking temperature), scenario B (two consumption events), scenario $C$ (prevalence in cattle: $2.6 \%$ ), and scenario $D$ (a hundred times lower bradyzoite concentration in cattle). 


\subsection{Deterministic susceptible-infected model}

From the deterministic SI model, with age of 80 and the fraction of $T$. gondii infection (7.9\%), the infection rate of $T$. gondii was estimated to be 0.005 per 1,000 persons per day (dashed horizontal line in Figure 6).

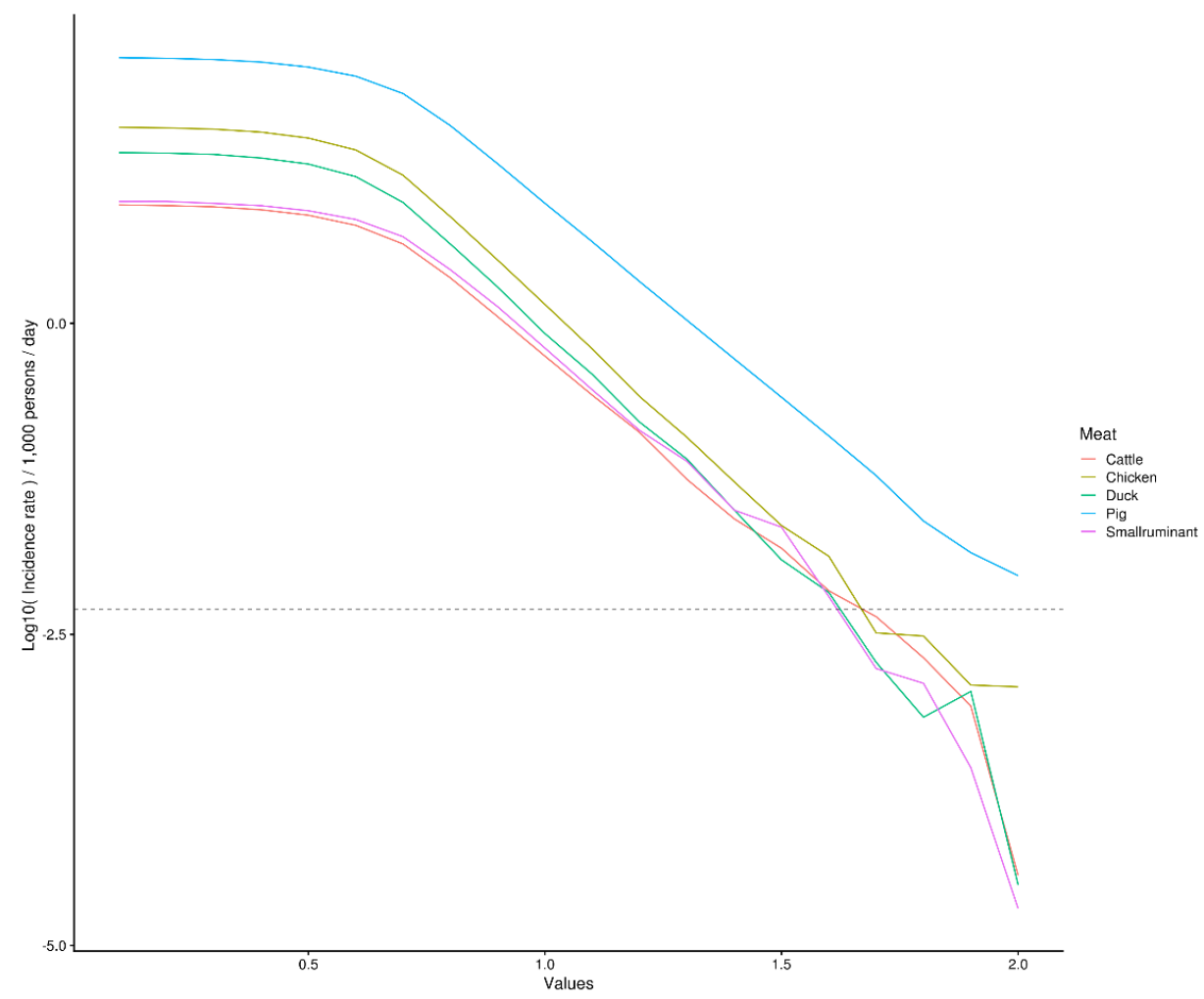

Figure 6. Sensitivity analysis results on final cooking temperature from the QMRA model for meat-borne $T$. gondii infections in China.

\section{Discussion}

This study presents the first QMRA model dealing with meat-borne $T$. gondii infection in mainland China. The mean incidence rates of human meat-borne $T$. gondii infection in one day in seven regions of mainland China were estimated and the relative importance of different meat types to human infections was compared.

From the QMRA model, the incidence rates in the general population and in women of childbearing age are calculated highest in the south-west of China and the lowest incidence rates were found in the north-east. Pork and chicken meat contributed most to the estimated meat-borne $T$. gondii infections in the seven 
regions. The relative attribution of different livestock species to meat-borne $T$. gondii infection is different from the Dutch QMRA model, which found that beef was the most important source of meat-borne T. gondii infection contributing $68 \%$ of the estimated infections in the Dutch population (Opsteegh et al., 2011a). More specifically, at product level, a raw minced beef product (filet americain) had the highest number of estimated infections. This difference may be due to the preference of different meat types and the amount of meat consumed in the two countries. Data from the National Bureau of Statistics of China showed that pork is the dominant type of meat consumed by the whole population, except for some religious groups, followed by poultry, beef and lamb, the average annual consumption amounts in 2015 were 20.1, 8.4, 1.6 and $1.2 \mathrm{~kg}$ respectively (National Bureau of Statistics of China, 2016). The consumption data we used in this study also showed the same preference. In contrast, in the Dutch QMRA model, $40 \%$ and $34 \%$ of the portions included (not taking the consumption amount into account) were of pork and beef origin, leaving $25.2 \%$ for pork/beef mixed products and $0.8 \%$ for sheep products (poultry products were left out because they were assumed to be heated to temperatures that inactivate the parasite).

Due to a lack of data, assumptions were made and several scenarios were performed to investigate the effect of these assumptions in our study. All scenario results showed that pork and chicken meat contributed more to the estimated infections than beef, small ruminant and duck meat (Fig. 5). However, the incidence rates were highly overestimated by the QMRA model. Even for the lowest incidence rate found in north-east (2.5/1,000 meat consumers/day), it means that more than $90 \%$ of the susceptible population will become infected after a year. This overestimation becomes more clear when comparing to the incidence rate of meat-borne $T$. gondii infection per day per 1,000 persons calculated from the SI model, the QMRA model overestimated the incidence rate at least thousand-fold. Moreover, the QMRA model only calculates the incidence rates for meat-borne infections, whereas the $\mathrm{SI}$ model includes all infection routes, and therefore the overestimation is even higher.

In the first scenario, the temperature profiles were increased by $10{ }^{\circ} \mathrm{C}$ and the estimated number of cases dropped compared to the base model. Applying the heating temperatures for US consumers to China may have contributed to the overestimation of number of estimated infections, if Chinese consumers generally heat their meat more thoroughly. Sensitivity analysis results also indicated that final cooking temperature was the most influential factor related to the estimated number of meat-borne $T$. gondii infections found in our model. This is consistent with the results from the other QMRA studies in the Netherlands and United States (Guo et al., 2017; Guo et al., 2016; Opsteegh et al., 2011a). In the cooking 
temperatures dataset, the final cooking temperatures recorded by American consumers had a wide range $\left(24\right.$ to $\left.138^{\circ} \mathrm{C}\right)$, including temperatures below $60{ }^{\circ} \mathrm{C}$. If final cooking temperatures increase around 1.7 times in the QMRA model (resulting in a minimum cooking temperature of $43^{\circ} \mathrm{C}$ ), the estimated incidence rate would be comparable to the results obtained from the SI model. However, in reality the differences on cooking temperatures would not consist of a multiplication, but rather an unknown distribution with a mean shifted to the right.

We believe that Chinese cooking techniques (e.g., stir-frying) uses higher heat than American's, and meat are usually cut into small pieces which means a higher core temperature will be easier to reach compared to a big piece of meat (e.g., steak). Therefore, using unrepresentative cooking temperature in our model likely underestimated the effect of $T$. gondii inactivation in meat and contributed to the overestimation of model outcome. Since the preference for cooking techniques of Chinese provinces can be influenced by many factors (e.g., socioeconomic status, religions and ethnic groups), information on regional and product specific cooking temperature from Chinese consumers is needed to improve the quantitative risk assessment of $T$. gondii infection through consumption of meat.

The second scenario, in which meat consumption amounts per livestock species were divided in two portions, showed that the incidence rates were higher in the situation with two consumption events. The fact that dividing the consumed amount over more portions results in a higher incidence suggests that more specific questions (e.g., consumption occasions and amount) should be added in the consumption survey for future QMRA studies. In order to investigate the impact of portion sizes and frequency on the estimated incidence rates and relative attribution of meat, an alternative approach was explored by re-structure the QMRA model (supplementary material C). The results obtained from the new approach showed that portion sizes and consumption frequency influence the incidence rate, but the relative attribution of the different livestock species remained similar compare to the base model.

In the third scenario, estimated infections due to consumption of beef were reduced when the prevalence of $T$. gondii based on direct detection (2.6\%) was used for cattle. Using the seroprevalence as a proxy for the prevalence of viable $T$. gondii in the base model was reasonable for pigs, small ruminant and poultry, because a good concordance was found between seropositivity and the presence of tissue cysts, but this concordance was not found in cattle (Opsteegh et al., 2016a; Opsteegh et al., 2016b; Opsteegh et al., 2011b) and therefore might be a source of overestimation. However, direct detection-based data on regional prevalence of $T$. gondii in cattle was not available, and only limited work has been published (Ge et 
al., 2014). Therefore, a prevalence of $2.6 \%$ based on direct detection results from a systematic review was used for all provinces in this scenario (Belluco et al., 2016). The ranking of relative importance of meat to $T$. gondii infection remained the same compared to the base model. Regional information on the prevalence of viable $T$. gondii in cattle is needed to get more accurate estimates.

In the fourth scenario, the relative importance of beef to $T$. gondii infection decreased after using a 100 times lower bradyzoite concentration in cattle than in other species. However, results from the base model and all the other scenarios already showed that beef was not the most important source of $T$. gondii infection in China.

Besides above-mentioned factors, the reasons for the overestimation of the QMRA model may be due to: first, in this study, we used quantitative data from a predilection site (hearts of naturally infected sheep) as input for the number of bradyzoites in unprocessed infected meat of all different consumption animals. In addition, tissue cysts were assumed to be evenly distributed in the muscles. The reality is more likely that tissue cysts are un-evenly distributed in different edible meat and differ between the different consumption animal species. Second, we assumed heat inactivation of $T$. gondii only occurred at consumer's kitchens. The concentration of $T$. gondii in meat cannot increase after the death of its hosts, therefore, after slaughter it can only be reduced, inactivated or remain unchanged. Studies showed that different processing methods, such as freezing, salting, heating and fermentation can reduce the number of viable bradyzoites in meat (Dubey, 1997; Dubey et al., 1990; Kotula et al., 1991). However, the available consumption data are only specified by animal species and not specified by product. Therefore, industrial processing methods such as salting cannot be implemented for specific products. In addition, consumption habits may also play an important role in $T$. gondii infection. For example, people living in the southwest of China have their own specific habits of eating raw or half-raw pork/beef (Zhou et al., 2011a), but this was not taken into account in our model due to a lack of quantitative data. Currently, the variation of estimated number of cases found in different regions is only due to the differences in consumed meat amount and seroprevalence in livestock. Besides, the overestimation could also be partly explained by not taking into account the amount of frozen meat in the model. Data showed that the import quantity of fresh/chilled/frozen pork, poultry, beef and small ruminant meat were 467.3, 421.3, 20.1 and 82.8 million kg in 2011 (Cheng et al., 2015). In the same year, the amount of pork, beef and mutton meat produced in China were 50,531, 6,475 and 3,931 million kg (National Bureau of Statistics of China, 2011). However, the actual proportion of frozen meat out of the total amount of imported or domestically produced meat was not clear, thus this 
information was not included in our model. Third, the single-hit probability in the dose-response relation used data from $T$. gondii type II, may not represent the $T$. gondii strains prevalent in China. Studies indicated that genotype Chinese 1 (ToxoDB\#9) is the predominant genotype found in cats, swine and human patients in China and it showed intermediately to highly virulence to mice (Chen et al., 2011; Wang et al., 2013; Zhou et al., 2011b). Further, the dose-response relation is based on mice experiment and mice are probably more susceptible than humans, however, it is not feasible to get more information on infectivity for humans from challenge studies or outbreaks.

In conclusion, we set up a QMRA model to estimate the incidence rate and the relative importance of different meat to human $T$. gondii infection in seven regions of mainland China for the first time. A wide variation of incidence rates of meatborne $T$. gondii infection was found in the general population and in women of childbearing age from the different regions of mainland China. Sensitivity analysis showed the incidence rate estimated from QMRA model would be comparable to the incidence rate from the $\mathrm{SI}$ models if cooking temperatures are around 1.7 times higher than the temperatures based on a dataset for American consumers. We conclude that unrepresentative cooking temperature and the possible overestimation of consumption frequency in our base model likely contribute to the overestimation when the model output is compared to epidemiological data. In general, pork and poultry meat were found to be the most important sources of human infections and heating temperature was found to be the most important parameter to influence the number of infections estimated by our model. Besides regional, product-specific cooking temperatures and consumption data, strainspecific dose-response relationship, and bradyzoite concentrations in infected livestock species other than sheep have been identified as key knowledge gaps in this study. Although many assumptions were made and the model overestimated the incidence considerably, the QMRA model identified influential parameters and significant gaps that exist in the data, this information can be used to guide future research work and help to focus on generating the most relevant data. When data gaps are filled, the structure of our QMRA can be used as a baseline model to support cost-effective decisions to reduce the disease burden of human toxoplasmosis. 


\section{Acknowledgements}

This study was carried out as part of the China Exchange Project supported by the National Key Research and Development Program of China (2017YFD0501300), the Royal Netherlands Academy of Arts and Sciences (530-5CDP21) and the Memorandum of Understanding programme funded by the Ministry of Health, Welfare and Sport in the Netherlands. We thank the anonymous reviewers for critically reviewing the manuscript and their many insightful comments and suggestions.

\section{Appendix A. Supplementary data}

Supplementary data associated with this article can be found, in the online version, at https://www.sciencedirect.com/science/article/pii/S2352352219300040. 


\section{REFERENCES}

Belluco, S., Patuzzi, I., Ricci, A., 2018. Bovine meat versus pork in Toxoplasma gondii transmission in Italy: A quantitative risk assessment model. Int. J. Food Microbiol. 269, 1-11.

Belluco, S., Mancin, M., Conficoni, D., Simonato, G., Pietrobelli, M., Ricci, A., 2016. Investigating the determinants of Toxoplasma gondii prevalence in meat: A systematic review and meta-regression. PLoS One 11, e0153856.

Chen, Z.W., Gao, J.M., Huo, X.X., Wang, L., Yu, L., Halm-Lai, F., Xu, Y.H., Song, W.J., Hide, G., Shen, J.L., Lun, Z.R., 2011. Genotyping of Toxoplasma gondii isolates from cats in different geographic regions of $C h i n a$. Vet. Parasitol. 183, 166-170.

Cheng, Y., Gao, Z., Seale, J., 2015. Changing structure of China's meat imports. J. Integr. Agric. 14, 1081-1091.

Condoleo, R., Rinaldi, L., Sette, S., Mezher, Z., 2017. Risk assessment of human toxoplasmosis associated with the consumption of pork meat in Italy. Risk Anal. 38: 1202-1222. doi:10.1111/risa.12934

Crotta, M., Limon, G., Blake, D.P., Guitian, J., 2017. Knowledge gaps in host-parasite interaction preclude accurate assessment of meat-borne exposure to Toxoplasma gondii. Int. J. Food Microbiol. 261, 95-101.

Deng, H., Devleesschauwer, B., Liu, M., Li, J., Wu, Y., van der Giessen, J.W.B., Opsteegh, M., 2018. Seroprevalence of Toxoplasma gondii in pregnant women and livestock in the mainland of China: a systematic review and hierarchical meta-analysis. Sci. Rep. 8, 6218.

Donders-Engelen, M.R., Heijden, L.J.M.v.d., Hulshof, K.F.A.M., 1997. Maten gewichten en codenummers. Wageningen Agricultural University, Wageningen.

Dong, Q.L., Barker, G.C., Gorris, L.G., Tian, M.S., Song, X.Y., Malakar, P.K., 2015. Status and future of quantitative microbiological risk assessment in China. Trends Food Sci. Technol. 42, 70-80.

Dubey, J.P., 1997. Survival of Toxoplasma gondii tissue cysts in $0.85-6 \% \mathrm{NaCl}$ solutions at 4-20 C. J. Parasitol. 83 , 946- 949.

Dubey, J.P., Kotula, A.W., Sharar, A., Andrews, C.D., Lindsay, D.S., 1990. Effect of high temperature on infectivity of Toxoplasma gondii tissue cysts in pork. J. Parasitol. 76, 201-204.

Dubey, J.P., 1998. Advances in the life cycle of Toxoplasma gondii. Int. J. Parasitol. 28, 1019-1024.

Dubey, J.P., Lindsay, D.S., Speer, C.A., 1998. Structures of Toxoplasma gondii tachyzoites, bradyzoites, and sporozoites and biology and development of tissue cysts. Clin. Microbiol. Rev. 11, 267-299.

Ecolab. (2008a). EcoSure 2007 cold temperature report. Available at: http://foodrisk.org/exclusives/ecosure/ (Accessed 23 November 2017).

Ecolab. (2008b). EcoSure2007 U.S. Cold Temperature Evaluation: Design and Summary Pages Technical Report, EcoSure (2008)

Elbez-Rubinstein, A., Ajzenberg, D., Dardé, M.-L., Cohen, R., Dumètre, A., Yera, H., Gondon, E., Janaud, J.-C., Thulliez, P., 2009. Congenital toxoplasmosis and reinfection during pregnancy: case report, strain characterization, experimental model of reinfection, and review. J. Infecti. Dis. 199, 280-285.

Ge, W., Sun, H., Wang, Z., Xu, P., Wang, W., Mu, G., Wei, F., Liu, Q., 2014. Prevalence and genotype of Toxoplasma gondii infection in cattle from Jilin Province, northeastern China. Vector Borne Zoonotic Dis. 14, 399402.

Guo, M., Lambertini, E., Buchanan, R.L., Dubey, J.P., Hill, D.E., Gamble, H.R., Jones, J.L., Pradhan, A.K., 2017. Quantifying the risk of human Toxoplasma gondii infection due to consumption of fresh pork in the United States. Food Control 73, Part B, 1210-1222.

Guo, M., Mishra, A., Buchanan, R.L., Dubey, J.P., Hill, D.E., Gamble, H.R., Jones, J.L., Du, X., Pradhan, A.K., 2016. Development of dose-response models to predict the relationship for human Toxoplasma gondii infection associated with meat consumption. Risk Anal. 36, 926-938.

Guo, M., Mishra, A., Buchanan, R.L., Dubey, J.P., Hill, D.E., Gamble, H.R., Pradhan, A.K., 2016. Quantifying the risk of human Toxoplasma gondii infection due to consumption of domestically produced lamb in the United States. J. Food Prot. 79, 1181-1187.

Havelaar, A.H., Galindo, A.V., Kurowicka, D., Cooke, R.M., 2008. Attribution of foodborne pathogens using structured expert elicitation. Foodborne Pathog. Dis. 5, 649-659.

Hoffmann, S., Devleesschauwer, B., Aspinall, W., Cooke, R., Corrigan, T., Havelaar, A., Angulo, F., Gibb, H., Kirk, M., Lake, R., Speybroeck, N., Torgerson, P., Hald, T., 2017. Attribution of global foodborne disease to specific foods: Findings from a World Health Organization structured expert elicitation. PLoS One 12, e0183641.

Hill, D., Dubey, J.P., 2002. Toxoplasma gondii: transmission, diagnosis and prevention. Clin. Microbiol. Infect. 8, 634-640. 
Innes, E.A., Bartley, P.M., Maley, S., Katzer, F., Buxton, D., 2009. Veterinary vaccines against Toxoplasma gondii. Mem. Ins. Oswaldo Cruz 104, 246-251.

Ji, Z.G., Chong. S., Ji, X.Y., Zhi, B.Z., 2005. A national survey on current status of the important parasitic diseases in human population. Chinese journal of parasitology \& parasitic diseases 23, 332-340.

Jiang, C., Li, Z., Chen, P., Chen, L., 2015. The seroprevalence of Toxoplasma gondii in Chinese population with cancer: A systematic review and meta-analysis. Medicine (Baltimore) 94, e2274.

Kotula, A.W., Dubey, J.P., Sharar, A.K., Andrews, C.D., Shen, S.K., Lindsay, D.S., 1991. Effect of freezing on infectivity of Toxoplasma gondii tissue cysts in pork. J. Food Pro. 54, 687-690.

Li, T., He, S., Zhao, H., Zhao, G., Zhu, X.Q., 2010. Major trends in human parasitic diseases in China. Trends Parasitol. 26, 264-270.

Montoya, J.G., Liesenfeld, O., 2004. Toxoplasmosis. Lancet 363, 1965-1976.

National Bureau of Statistics of China, 2011. Statistical yearbooks. Available at: http://www.stats.gov.cn/tjsi/ndsi/2011/indexeh.htm (Accessed 23 November 2017).

National Bureau of Statistics of China, 2016. Statistical yearbooks. Available at: http://www.stats.gov.cn/tjsi/ndsj/2016/indexeh.htm (Accessed 23 November 2017).

Opsteegh, M., Kortbeek, T.M., Havelaar, A.H., van der Giessen, J.W., 2015. Intervention strategies to reduce human Toxoplasma gondii disease burden. Clin. Infect. Dis. 60, 101-107.

Opsteegh, M., Langelaar, M., Sprong, H., den Hartog, L., De Craeye, S., Bokken, G., Ajzenberg, D., Kijlstra, A., van der Giessen, J., 2010. Direct detection and genotyping of Toxoplasma gondii in meat samples using magnetic capture and PCR. Int. J. Food Microbiol. 139, 193-201.

Opsteegh, M., Maas, M., Schares, G., Van der Giessen, J., on behalf of the consortium, 2016a. Relationship between seroprevalence in the main livestock species and presence of Toxoplasma Gondii in Meat (GP/EFSA/BIOHAZ/2013/01). An extensive literature review. Final report. EFSA.

Opsteegh, M., Prickaerts, S., Frankena, K., Evers, E.G., 2011a. A quantitative microbial risk assessment for meatborne Toxoplasma gondii infection in The Netherlands. Int. J. Food Microbiol. 150, 103-114.

Opsteegh, M., Schares, G., Blagac, R., van der Giessen, J., on behalf of the consortium, 2016b. Experimental studies on Toxoplasma gondii in the main livestock species (GP/EFSA/BIOHAZ/2013/01) Final report. EFSA.

Opsteegh, M., Teunis, P., Zuchner, L., Koets, A., Langelaar, M., van der Giessen, J., 2011b. Low predictive value of seroprevalence of Toxoplasma gondii in cattle for detection of parasite DNA. Int. J. Parasitol. 41, 343354.

Pappas, G., Roussos, N., Falagas, M.E., 2009. Toxoplasmosis snapshots: global status of Toxoplasma gondii seroprevalence and implications for pregnancy and congenital toxoplasmosis. Int J Parasitol 39, 13851394.

R Development Core Team, 2006. R: A Language and Environment for Statistical Computing. R Foundation for Statistical Computing, Vienna, Austria.

Torgerson, P.R., Devleesschauwer, B., Praet, N., Speybroeck, N., Willingham, A.L., Kasuga, F., Rokni, M.B., Zhou, X.N., Fevre, E.M., Sripa, B., Gargouri, N., Furst, T., Budke, C.M., Carabin, H., Kirk, M.D., Angulo, F.J., Havelaar, A., de Silva, N., 2015. World Health Organization estimates of the global and regional disease burden of 11 foodborne parasitic diseases, 2010: A data synthesis. PLoS Med 12, e1001920.

Torgerson, P.R., Mastroiacovo, P., 2013. The global burden of congenital toxoplasmosis: a systematic review. Bull. World. Health. Organ. 91, 501-508.

Wang, L., Chen, H., Liu, D., Huo, X., Gao, J., Song, X., Xu, X., Huang, K., Liu, W., Wang, Y., Lu, F., Lun, Z.R., Luo, Q., Wang, X., Shen, J., 2013. Genotypes and mouse virulence of Toxoplasma gondii isolates from animals and humans in China. PLoS One 8, e53483.

Weiss, L.M., Dubey, J.P., 2009. Toxoplasmosis: A history of clinical observations. Int. J. Parasitol. 39, 895-901.

Wu, Y., Li, X., 2015. The fourth China total diet study. Chemical Industry Press, Beijing, China.World Health Organisation (WHO), Risk assessment. Available at: https://www.who.int/foodsafety/risk- analysis/riskassessment/en/ (Accessed 18 December 2018).

Yang, G., Wang, Y., Zeng, Y., Gao, G.F., Liang, X., Zhou, M., Wan, X., Yu, S., Jiang, Y., Naghavi, M., Vos, T., Wang, H., Lopez, A.D., Murray, C.J., 2013. Rapid health transition in China, 1990-2010: findings from the global burden of disease study 2010. Lancet 381, 1987-2015.

Zhang, N.Z., Wang, M., Xu, Y., Petersen, E., Zhu, X.Q., 2015. Recent advances in developing vaccines against Toxoplasma gondii: an update. Expert Rev. Vaccines 14, 1609-1621.

Zhou, P., Chen, Z., Li, H.L., Zheng, H., He, S., Lin, R.Q., Zhu, X.Q., 2011a. Toxoplasma gondii infection in humans in China. Parasit. Vectors 4, 165.

Zhou, P., Sun, X.T., Yin, C.C., Yang, J.F., Yuan, Z.G., Yan, H.K., Zhu, X.Q., Zou, F.C., 2011b. Genetic characterization of Toxoplasma gondii isolates from pigs in southwestern China. J. Parasitol. 97, 1193-1195 


\section{Chapter}

\section{Digging into Toxoplasma gondii infections via soil: a quantitative microbial risk assessment approach}

Science of the Total Environment, 2020

Huifang Deng ${ }^{1}$, Kitty E. Exel ${ }^{1,2}$, Arno Swart ${ }^{1}$, Axel A. Bonačić Marinovic ${ }^{1}$, Cecile Dam-Deisz ${ }^{1}$, Johanna W. B. van der Giessen ${ }^{1}$, Marieke Opsteegh ${ }^{1}$

Authors' affiliations:

${ }^{1}$ Centre for Infectious Disease Control - Zoonoses and Environmental Microbiology, National Institute for Public Health and the Environment, Bilthoven, the Netherlands

${ }^{2}$ Faculty of Veterinary Medicine, Utrecht University, Utrecht, the Netherlands 


\begin{abstract}
Background: Soil has been identified as an important source of exposure to a variety of chemical and biological contaminants. Toxoplasma gondii is one of those potential biological contaminants associated with serious health effects in pregnant women and immunocompromised patients. Gardening or consumption of homegrown vegetables may present an important route of $T$. gondii infection via accidental ingestion of soil. In the Netherlands, there is quantitative information on the risk of $T$. gondii infection via meat products, but not on the risk of infection through soil.
\end{abstract}

Methods: The objective of this study was to develop a quantitative microbial risk assessment (QMRA) model for estimating the risk associated with $T$. gondii exposure via accidental soil ingestion in the Netherlands. In order to obtain the needed information, a magnetic capture method for detection of $T$. gondii oocysts in soil samples was developed, and $T$. gondii DNA was detected using qPCR targeting the 529 bp repeat element.

Results: The method was shown to provide $95 \%$ probability of detection $(95 \% \mathrm{Cl}$ : $88-100 \%$ ) when at least 34 oocysts are present in 25 grams of soil. T. gondii DNA was detected in 5 of 148 soil samples with interpretable results (3\%, $95 \% \mathrm{Cl}: 1.5-$ 7.7\%). Results for 18 samples were not interpretable due to PCR inhibition. The estimated amount of oocysts presented in qPCR positive samples was quantified by a linear model, and the amount varied from 8 to 478 in 25 grams of soil. The estimated incidence rate of $T$. gondii infection from the QMRA model via soil varied from 0.3 to 1.8 per 1,000 individuals per day.

Conclusions: Several data gaps (e.g., soil contamination/ingestion and oocysts viability) have been identified in this study, the structure of the model can be applied to obtain more accurate estimates of the risk of $T$. gondii infection via soil when data become available. 


\section{Introduction}

Toxoplasma gondii is a widely prevalent single-celled parasite, infecting around one third of the human population. Humans become infected mainly through consumption of raw or undercooked meat containing viable $T$. gondii tissue cysts, or ingestion of oocysts via contaminated water, soil, or food (Tenter et al., 2000). Although infections are generally asymptomatic in humans, they can cause abortion in pregnant women, ocular disease, hydrocephalus or intracranial calcifications in congenitally infected children, ocular toxoplasmosis in acquired infections and severe symptoms and even death in severely immunocompromised patients (Jones et al., 1999). Based on data collected from 108 countries in a systematic review, the estimated annual global incidence of congenital toxoplasmosis (CT) was 1.5 cases per 1,000 live births, and the burden of CT was estimated to be 1.2 million disability-adjusted life years (95\% Cl: 0.76-1.90) (Torgerson and Mastroiacovo, 2013).

Cats (domestic cats and wild felids), the only known definitive hosts of the parasite, play an important role in $T$. gondii transmission. These definitive hosts acquire $T$. gondii infection mainly through infected prey (e.g., rodents and birds) (Dubey, 2006), and are able to excrete millions of oocysts into the environment (Dubey et al., 1970). After primary infection, sexual reproduction of $T$. gondii occurs in the intestine of the definitive host and unsporulated oocysts are produced and excreted in the faeces (Miller et al., 1972). The excretion of T. gondii oocysts lasts for 1 to 2 weeks (Dubey, 1995) and the process of sporogony, which leads to the development of infectious oocysts, occurs 1 to 3 days after deposition depending on humidity and temperature (Dubey et al., 1970). Sporulated T. gondii oocysts are environmentally resistant and can survive for months in soil and water (Frenkel et al., 1975). However, oocysts can lose the ability to sporulate when exposed to constant freezing at $-6{ }^{\circ} \mathrm{C}$ for 7 days or $-21^{\circ} \mathrm{C}$ for one day (Frenkel and Dubey, $1973)$, and heating $\left(50^{\circ} \mathrm{C}\right)$ for 10 minutes (Dubey et al., 1970). Usually, cats remain immune to new $T$. gondii infection for life after the initial infection, and repeated oocyst excretion by cats is unlikely. However, under experimental settings reexcretion was reported when a second challenge with a new strain of $T$. gondii was applied 6 years later after the primary infection (Dubey, 1995). Oocysts can be dispersed in the environment by different factors, such as wind, run-off and arthropods. Therefore, $T$. gondii oocysts become a potential source of infection for all warm-blooded animals (intermediate hosts) via contaminated vegetables, water and soil (Aubert and Villena, 2009; Caradonna et al., 2017; Wells et al., 2015).

To be able to implement efficient intervention strategies to reduce the disease burden of toxoplasmosis, it is essential to know the relative importance of the 
different sources of infection. There are two main transmission modes for postnatal human infection: via consumption of raw/undercooked meat containing viable tissue cysts, and via ingestion of oocysts present in the environment, e.g., from contaminated vegetables, water and soil (Tenter et al., 2000). Several approaches to attribute disease burden to various sources have been used for $T$. gondii in recent years, e.g., outbreak investigation, epidemiological methods, risk assessment models, and expert elicitations (EFSA, 2018; Mughini-Gras et al., 2018). Several acute toxoplasmosis outbreaks in humans have been reported worldwide, e.g., an outbreak with 809 confirmed cases occurred in Brazil in 2018, which was considered the largest outbreak ever described in the world (Minuzzi et al., 2020). Among those outbreaks, a few of them were linked to oocysts (Bowie et al., 1997; Coutinho et al., 1982; de Moura et al., 2006; Ekman et al., 2012). Exposure to soil has long been identified as an important risk factor for human infection (Cook et al., 2000; Jones et al., 2006; Jumaian, 2005; Shapiro et al., 2019). For instance, an epidemiological case-control study in pregnant women conducted in six large European cities showed that $6-17 \%$ of infections could be attributed to soil contact, while meat contributed to $30-63 \%$ of $T$. gondii infection (Cook et al., 2000). However, to relate exposure to infection, epidemiological studies best focus on recent infections. As T. gondii infection often passes asymptomatically and severe sequela can occur years later, or with non-specific symptoms, a long-term prospective study is needed to identify and include enough cases to provide sufficient statistical power. In this context, quantitative microbial risk assessments (QMRA) are more feasible to estimate the attribution disease burden to the sources. A few QMRA studies concerning meat-borne $T$. gondii infection have been published (Belluco et al., 2018; Condoleo et al., 2017; Crotta et al., 2017; Deng et al., 2020; Guo et al., 2017; Opsteegh et al., 2011). However, no QMRA models include transmission via oocysts so far. Consequently, the relative importance of oocyst-borne $T$. gondii infection cannot be compared to results obtained from QMRA studies for meat-borne infection. Expert elicitations estimated that foodborne attribution proportions within the European regions ranged from $45 \%$ to $61 \%$, while soil and water contact attributed $18-37 \%$ and $15-23 \%$ respectively (Hald et al., 2016). In the Netherlands, experts indicated that $56 \%$ of $T$. gondii infections are food-borne and $36 \%$ via environmental contamination (Havelaar et al., 2008). Expert elicitations is the most frequently applied approach for attributing $T$. gondii infection to major transmission routes and modes, however, it has the drawback that it is not transparent because the data driving the knowledge of the experts are not specified. In addition, a serological assay to detect sporozoite specific antibodies in recently infected individuals to study oocyst-borne $T$. gondii infections has been developed. High levels of oocyst acquired infections in the United States of America (78\%) and Chile (45\%) were reported using this test 
(Boyer et al., 2011). So far, no such study has been conducted in Europe (EFSA, 2018).

It was our aim to quantify the risk of $T$. gondii infection via accidental ingestion of soil by developing a QMRA model. This requires quantitative data on soil ingestion and the presence of $T$. gondii in soil. Particularly among young children, the potential of exposure to $T$. gondii by this mode is likely greater than adults because of behavior. Several studies have been conducted for estimating the daily intake of soil (Binder et al., 1986; Calabrese et al., 1989; Davis et al., 1990; Lin et al., 2017; Stanek and Calabrese, 1995; Stanek et al., 1997; van Wijnen et al., 1990). It generally involves measuring the presence of tracer elements that are assumed to be poorly absorbed in the gut, in the soil/food and later in the faeces of an individual.

Due to the difficulties in recovering, detecting and quantifying the number of oocysts in environmental samples (Dumetre and Darde, 2003), only a limited number of studies concerning the $T$. gondii oocyst contamination rate in soil have been published so far (Afonso et al., 2008; de Wit et al., 2020; dos Santos et al., 2010; Du, et al., 2012a, 2012b; Gotteland, Gilot-Fromont, et al., 2014; Lass et al., 2009; Liu et al., 2017). Currently, microscopical examination and molecular assays are the most commonly used methods for detecting $T$. gondii oocysts in soil samples. Usually, sucrose/sodium nitrate flotation to purify and separate oocysts from the complicated matrices was done before microscopical examination, and then followed by molecular confirmation targeting the B1 gene and or the $529 \mathrm{bp}$ repeat element (including both conventional and real-time, quantitative PCR) or bioassay (Lélu et al., 2011). A main drawback of this approach is the low recovery percentage of flotation. Another potential approach is the immunomagnetic detection of $T$. gondii oocysts, a technique based on the use of magnetic beads conjugated to specific monoclonal antibodies against the cell surface markers of the pathogen (Dumetre and Darde, 2005; Hohweyer et al., 2016; Sousa et al., 2020) This method is commonly used for the detection of Cryptosporidium spp. and Giardia spp. in environmental samples (Schets et al., 2008). However, since no suitable monoclonal antibodies are commercially available for $T$. gondii, this method cannot be implemented easily (Jones \& Dubey, 2010).

In 2010, a sequence-specific magnetic capture method has been developed by Opsteegh et al. (2010) to detect T. gondii DNA in meat samples. Briefly, a crude DNA isolate is prepared from $100 \mathrm{~g}$ meat sample and $T$. gondii DNA is specifically captured using biotin-labelled oligonucleotide and streptavidin labelled magnetic beads. Afterwards, T. gondii DNA is detected by qPCR targeting the 529 bp repeat 
element. Although PCR does not demonstrate infectivity, the method is more rapid than mouse bioassay (Opsteegh et al., 2010). As oocysts are likely concentrated in or near the defecation sites of the definitive hosts (Bastien et al., 2019), rather than homogeneously distributed in soil, the ability to test a relatively large amount of material per sample compared to the sample size used in commercial DNA extraction kits make this MC-qPCR a promising method for detecting $T$. gondii in soil. At the same time, this sequence-specific magnetic capture approach can overcome PCR inhibition from complex matrices, and does not depend on monoclonal antibodies to capture the intact parasites.

The primary objective of this study was to develop a QMRA model to estimate the risk associated with $T$. gondii exposure via accidental ingestion of soil from residential gardens in the Netherlands. In order to obtain exposure data for the risk assessment model, the sequence-specific magnetic capture based method developed by Opsteegh et al. (2010) was modified and used to estimate the number of $T$. gondii oocysts in soil samples.

\section{Materials and methods}

\subsection{Quantitative risk assessment (conceptual model)}

The risk assessment model uses a stepwise procedure to estimate the risk associated with $T$. gondii infection through exposure to soil from home gardens (Fig. 1). It consists of an exposure module, a dose-response module and a risk characterization module. The whole population was categorized into two exposure groups based on age: group one ( $\leq 10$ years), and group two (>10 years), the incidence rates and number of new infections per day were calculated for both age categories.

\subsubsection{Exposure assessment}

The daily exposure to viable $T$. gondii via ingestion of soil (Dose) can be calculated using Equation (1). Where $I n_{\text {soil }}$ is soil intake $(\mathrm{mg}), C_{\text {oocysts }}$ is concentration of $T$. gondii oocysts per $\mathrm{mg}$ of soil, $\mathrm{V}_{\text {oocyst }}$ is the fraction of viable $T$. gondii oocysts.

Dose $=I n_{\text {soil }} \times C_{\text {oocysts }} \times V_{\text {oocyst }}$

Soil intake for age group one was estimated based on data from van Wijnen et al. (1990). The study measured the amount of three tracer elements (titanium, aluminum, and acid insoluble residue) in soil and feces from Dutch children. The soil intake was calculated using a limiting tracer method, which assumed that the maximum amount of soil intake cannot be higher than the lowest estimate of the 


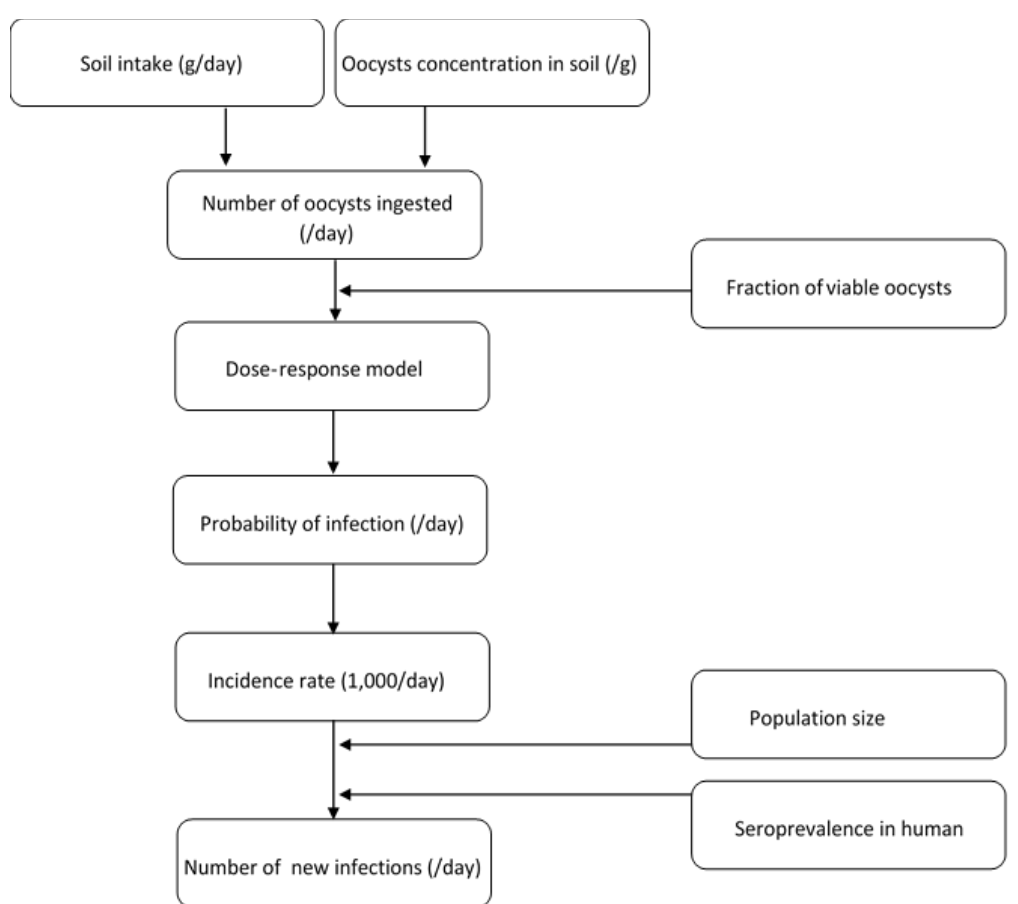

Figure 1. The conceptual quantitative risk assessment model for $T$. gondii infection via accidental soil ingestion in the Netherlands.

tracers. A control group (hospitalized children) was included for estimating the dietary background of the tracers. The estimates for soil were derived from outdoor, indoor or both sources. A log-normal distribution was assumed for soil intake, mean $(102 \mathrm{mg})$ and SD $(1.7 \mathrm{mg})$ of the distribution were obtained by using approximate Bayesian computation (ABC) (Sunnåker et al., 2013). In the paper by van Wijnen et al. (1990), only summary statistics such as "number of children, geometric mean, geometric standard deviation" were presented. Since it is hard to directly derive the parameters of our postulated log-normal distribution for overall intake, we resorted to $A B C$. Briefly, uninformative priors for the parameters were established and samples were drawn from them. Using these samples, data in the same form as van Wijnen et al. (1990) was simulated. Only those samples that generate data close to the true data were retained. In the end, the accepted samples formed the posterior distribution. The estimated mean $(93 \mathrm{mg})$ and SD (20 mg) of the control group were used for correcting the dietary background of soil ingestion, by subtracting samples of this distribution from samples of the calculated posterior distribution, and were used in the QMRA calculation. If negative values resulted, they were interpreted as 'no exposure'. Daily soil intake for those people were zero in the model. Soil intake information among adults is 
extremely limited, thus an assumption was made that the amount of daily soil intake for adults was half of the amount from children (EPA, 2011). The concentration of $T$. gondii oocysts in soil was randomly sampled from the estimated concentration of $T$. gondii in $25 \mathrm{~g}$ of soil with replacement (data from this study), dividing by 25 and multiplying with the soil exposure in grams as calculated in the previous step. The fraction of viable $T$. gondii oocysts was set to 0.5 for our base model, and two scenarios (with the fractions of 0.2 and 1 separately) were included in the calculation.

\subsubsection{Dose-response model}

For calculating the risk of infection of a person per day $\left(p_{\text {day }}\right)$, a dose-response relation is needed. In this study, we used a modified Beta Poisson dose-response model ( $\alpha=0.38, \beta=0.14$ ) developed by Bonačić Marinović et al. (2019). The model was built based on published experimental data from mice, rats and pigs, because there was no data available for humans. Since humans, mice, rats and pigs are all mammalian intermediate hosts of the parasite, we assumed that the doseresponse relation in humans is similar to that of those animals. The dose-response model assumes that every single oocyst is independently able to cause infection. The estimated probability of successfully initiating $T$. gondii infection with one oocyst was 0.46 (95\% Cl: 0.31-0.57) (Bonačić Marinović et al., 2019).

\subsubsection{Risk characterization}

The status of $T$. gondii infection (yes or no) via ingestion of soil was drawn from a Bernoulli distribution with the probability of infection per day obtained from the dose-response relationship. The incidence rate was then calculated using the total number of infections divided by the number of iterations $(10,000)$ in the model. Finally, the estimated infections in the Dutch population was calculated by multiplying the incidence rate with the population size. Since we were only interested in the number of new infections, we had to take immunity from previous infections into account. Therefore, the reported seroprevalence for the Dutch population was used (Hofhuis et al., 2011). Hence, the fraction of the naive population of 0.91 or 0.70 (1- seroprevalence) was multiplied with the population sizes for age group one $(1,989,559)$ and two $(15,091,948)$ (CBS, 2018).

The model was implemented in $R$ version 3.3.1 ( $R$ Development Core Team, 2006), and $R$ code can be found in the supplementary material $A$.

\subsection{Detection of T. gondii in soil}

\subsubsection{Soil sampling}

Soil samples were collected between August 2018 and November 2019 at different residential gardens and a limited number of playgrounds located in the 
Netherlands using convenience sampling. Colleagues from the Dutch National Institute for Public Health and Environment (RIVM) and their family/friends were asked to supply a soil sample from their garden. Every sample consisted of five subsamples, which were taken from upper $5 \mathrm{~cm}$ of the surface within one square meter. Around $100 \mathrm{~g}$ of soil was collected for each sample. In addition to providing a soil sample, the volunteers answered an online questionnaire with 17 questions (e.g., the location of sample and presence of cats), and signed an informed consent form (supplementary material B). All samples were sent back to RIVM and stored at $-20{ }^{\circ} \mathrm{C}$ until testing.

\subsubsection{Magnetic capture qPCR method}

\subsubsection{Preparation of crude DNA extract}

For every sample, $25 \mathrm{~g}$ of soil was randomly taken and equally divided over two 50$\mathrm{ml}$ conical centrifuge tubes, and $25 \mathrm{ml}$ demineralized water was added to each tube. Next, freeze-thaw cycles were performed by three rounds of putting the samples on dry ice for $1 \mathrm{~h}$ followed by $1 \mathrm{~h}$ in a water bath $( \pm 50 \stackrel{\circ}{ })$ ), to break the bi-layered wall of the oocysts. After the freeze-thaw cycles, the soil samples, consisting of two tubes each, were combined into a stomacher bag with filter (Seward, Worthing, UK). The samples were incubated overnight with $200 \mathrm{ml}$ lysis buffer (100 mM Tris- $\mathrm{HCl} \mathrm{pH} 8.0,5 \mathrm{mM}$ EDTA, $200 \mathrm{mM} \mathrm{NaCl}, 0.4 \%$ SDS) and proteinase $\mathrm{K}(20 \mathrm{mg} / \mathrm{ml}$, Qiagen, Venlo, the Netherlands) in a water bath set at 55 oC. After incubation, $50 \mathrm{ml}$ of crude DNA extract was transferred to a clean $50-\mathrm{ml}$ tube for magnetic capture.

For each isolation, negative and positive controls were included. Soil used for preparing the controls were from a garden where several soil samples were tested negative before the initial collection of samples. The positive controls were soil samples spiked with dilutions of a counted $T$. gondii oocyst stock (stored/diluted in water suitable for PCR).

\subsubsection{Removal of biotin}

The protocol for the sequence-specific magnetic capture was adapted from Opsteegh et al. (2010). First, crude DNA extract $(50 \mathrm{ml}$ ) was centrifuged at 3,500 $\times$ $\mathrm{g}$ for $20 \mathrm{~min}$, and supernatant $(12 \mathrm{ml})$ was transferred to a $15-\mathrm{ml}$ polypropylene tube. The supernatant was then heated at $100 \stackrel{\circ}{\circ}$ for $10 \mathrm{~min}$ in a water bath to inactivate the proteinase $\mathrm{K}$. After, cooling down to a maximum temperature of 40 oC, $50 \mu \mathrm{l}$ of PBS-washed streptavidin-sepharose (GE Healthcare, Hoevelaken, the etherlands) was added to the sample. Then, after rotating (10 rpm) the sample for $15 \mathrm{~min}$ at room temperature for streptavidin-biotin binding, the DNA extract was 
centrifuged at 3,500 $\times \mathrm{g}$ for $10 \mathrm{~min}$. Last, $10 \mathrm{ml}$ biotin-free supernatant was transferred to a new 15-ml polypropylene tube.

\subsubsection{Sequence-specific magnetic capture}

Ten picomoles of Tox-CapF and Tox-CapR capture oligonucleotides (Opsteegh et al., 2010) were added to the biotin-free DNA extract, following which $15 \mathrm{~min}$ heating in the water bath at $95 \stackrel{\circ}{\circ}$ to denature DNA, and then cooled down for 15 min for hybridization by rotating at $10 \mathrm{rpm}$ at room temperature. Afterwards, 80 $\mu \mathrm{l}$ of washed M-270 streptavidin dynabeads (Invitrogen, Landsmeer, the Netherlands) and $2 \mathrm{ml}$ of $5 \mathrm{M} \mathrm{NaCl}$ were added to the sample, and incubated for $45 \mathrm{~min}$ at room temperature, while rotating at $10 \mathrm{rpm}$. After incubation, the sample was placed in a Dynal ${ }^{\circ} \mathrm{MPC}^{\mathrm{Tm}}$-1 magnet (Invitrogen, Landsmeer, the Netherlands) for $5 \mathrm{~min}$ to isolate the complex of streptavidin bead and biotinlabelled capture oligonucleotide hybridized $T$. gondii DNA from the supernatant, and the supernatant was discarded using a pastette (Alpha laboratories, Hampshire, United Kingdom). The complex was resuspended, washed three times in B\&W buffer, and resuspended in $100 \mu \mathrm{l}$ water suitable for PCR (Sigma-Aldrich, Zwijndrecht, the Netherlands). The complex was heated at $100 \stackrel{\circ}{C}$ for $10 \mathrm{~min}$ to release the $T$. gondii DNA from the beads. Lastly, the tube was placed in a Dynal $\mathrm{MPC}^{\mathrm{T}}$-S magnet (Invitrogen, Landsmeer, the Netherlands) and the supernatant containing the $T$. gondii DNA was immediately transferred to a clean $1.5-\mathrm{ml}$ tube.

\subsubsection{Real-time quantitative PCR}

Real-time quantitative PCR (qPCR) has been performed on the 529 bp repeat element, with minor modifications to the protocol previously described by Opsteegh et al. (2010). The qPCR was performed in a 96-wells plate using a LightCycler 480 thermal-cycler instrument (Roche, Almere, the Netherlands). The LightCycler ${ }^{\circ} 480$ Probes Master kit was used, and the $20 \mu \mathrm{l}$ reaction volume consisted of $10 \mu \mathrm{l}$ master mix and $8 \mu \mathrm{l}$ extracted DNA, $1 \mu \mathrm{l}$ competitive internal amplification control (CIAC), and $1 \mu \mathrm{l}$ primer/probe mix (14 pmol/ $\mu \mathrm{l}$ Tox-9F, 14 $\mathrm{pmol} / \mu \mathrm{l}$ Toxo-11R, $2 \mathrm{pmol} / \mu \mathrm{l} \mathrm{Tox}-\mathrm{TP}-1$, and $4 \mathrm{pmol} / \mu \mathrm{l} \mathrm{ClAC}$-probe). Thermocycling began at $95 \stackrel{\circ}{\circ}$ for $10 \mathrm{~min}$ followed by 45 amplification cycles consisting of $1 \mathrm{~s}$ at

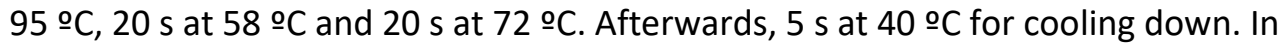
each qPCR run, a T. gondii DNA standard series varying from 0.03 to $300 \mathrm{pg}$ was added to generate the standard curve, to check PCR efficiency (>1.85) and used for quantification.

A CIAC was added to the PCR mix for checking PCR inhibition and thereby identify potential false negative PCR results, as described by Opsteegh et al. (2010). However, the label of the CIAC-probe has been modified to avoid the need for color 
compensation. With the new label (ATTO-647N) the emission of the FAM-labelled T. gondii-probe $(530 \mathrm{~nm})$ and CIAC-probe $(660 \mathrm{~nm})$ do not overlap.

Samples with a Cq value less than 40 and a smooth exponential-amplification curve similar to the positive controls were considered positive. When samples had no $\mathrm{Cq}$ value in the $T$. gondii reaction, but CIAC amplification was successful, they were considered negative. A sample was considered 'not determined' if there was no amplification of $T$. gondii DNA and $\mathrm{CIAC}$, since these samples were potentially false negative due to PCR inhibition. Inhibited samples were additionally tested by qPCR using a 1:10 dilution of the DNA.

\subsubsection{Data analysis}

\subsubsection{Detection probability of the modified magnetic capture qPCR}

Several $5 \mu \mathrm{l}$ aliquots were prepared from an oocyst stock and then applied to counting slides for counting the number of oocysts under microscope. The concentration of the oocyst stock was then calculated. Different dilution series were prepared from the oocyst stock for the spiking experiments. Experimental soil replicates that spiked with an estimated numbers of oocysts ranging from 1 to 500 oocysts or concentrations of $T$. gondii DNA ranging from 0.03-300 pg were tested. The replicates were then scored as either detectable (positive) or nondetectable (negative). In order to determine the sensitivity of the modified magnetic capture qPCR method, the oocyst-count dependent detection probability (Evers et al., 2010) was estimated by using a probit analysis in R (R Development Core Team, 2006) (supplementary material A). The probit model (Equation 2) assumes that the probability of detection is related to the $\log _{10}$-transformed concentration data as the cumulative normal distribution. Thus, the probability of detection was inverse normal transformed (called probits, short for "probability units").

$\operatorname{Probit}(p)=\beta_{0}+\beta_{1} \times \log _{10}($ Oocysts $)$

\subsubsection{Quantification of oocysts}

In real-time qPCR the $\mathrm{Cq}$ values decrease linearly with an increasing $\log _{10^{-}}$ transformed DNA concentration. A linear model was fitted to results obtained from T. gondii DNA dilution series and samples spiked with known numbers of oocysts in order to quantify the DNA or parasite concentration in samples (Equation 3).

$$
C q=\beta_{0}+\left(\beta_{1} \times \text { type }\right)-\left(\beta_{2} \times \log _{10}(\text { Oocysts or DNA })\right)
$$


Where type $=0$ for DNA and type $=1$ for oocysts. This model assumes a shared slope for both oocysts and DNA samples. This was motivated by the observation that many $\mathrm{Cq}$ values for the oocyst samples were truncated at 40 , hampering proper fitting. Setting a shared slope is biologically plausible and yields a more satisfactory fit.

\subsubsection{Soil survey}

Soil samples collected at different residential gardens and playgrounds located in the Netherlands were tested using the developed magnetic capture qPCR. Proportion of 'not determined' and positive samples with $95 \% \mathrm{Cl}$ was calculated. Descriptive statistics for questionnaire data were obtained.

\section{Results}

\subsection{Detection probability of the modified magnetic capture qPCR}

The optimized detection method was applied to eight concentration groups of samples spiked with oocysts in $25 \mathrm{~g}$ soil (Fig. 2), and the qPCR to five T. gondii DNA concentrations. The $95 \%$ detection probability of the MC-qPCR was at least 34 oocysts in $25 \mathrm{~g}$ of soil ( $95 \% \mathrm{Cl}: 88-100 \%$ ) (Equation 4), and $0.26 \mathrm{pg}$ of $T$. gondii DNA (95\% Cl: 86-100\%) for the qPCR (Equation 5) (Fig. 3).

$$
\begin{aligned}
& \operatorname{Probit}\left(p_{0}\right)=-1.2+1.8 \times \log _{10}(\text { Oocysts }) \\
& \operatorname{Probit}\left(p_{1}\right)=2.2+1.1 \times \log _{10}(D N A)
\end{aligned}
$$

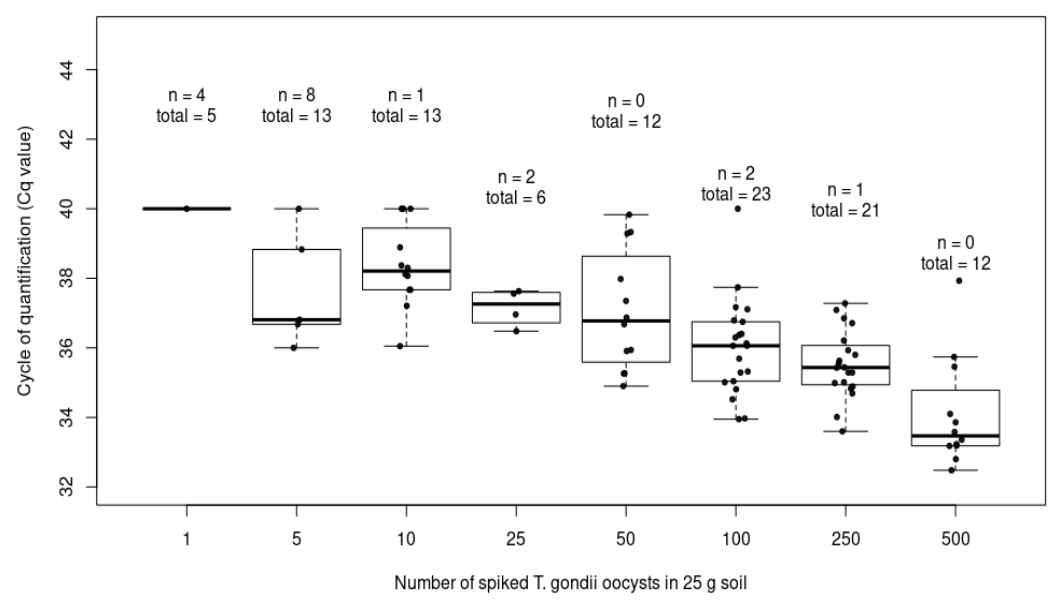

Figure 2. Cycle of quantification values of different number of $T$. gondii oocysts spiked in 25 grams of soil detected using magnetic capture qPCR ( $n$ : number of non-detectable samples, total: total number of spiked soil samples; Cq values were censored at around 40 due to detection limit of the PCR machine). 


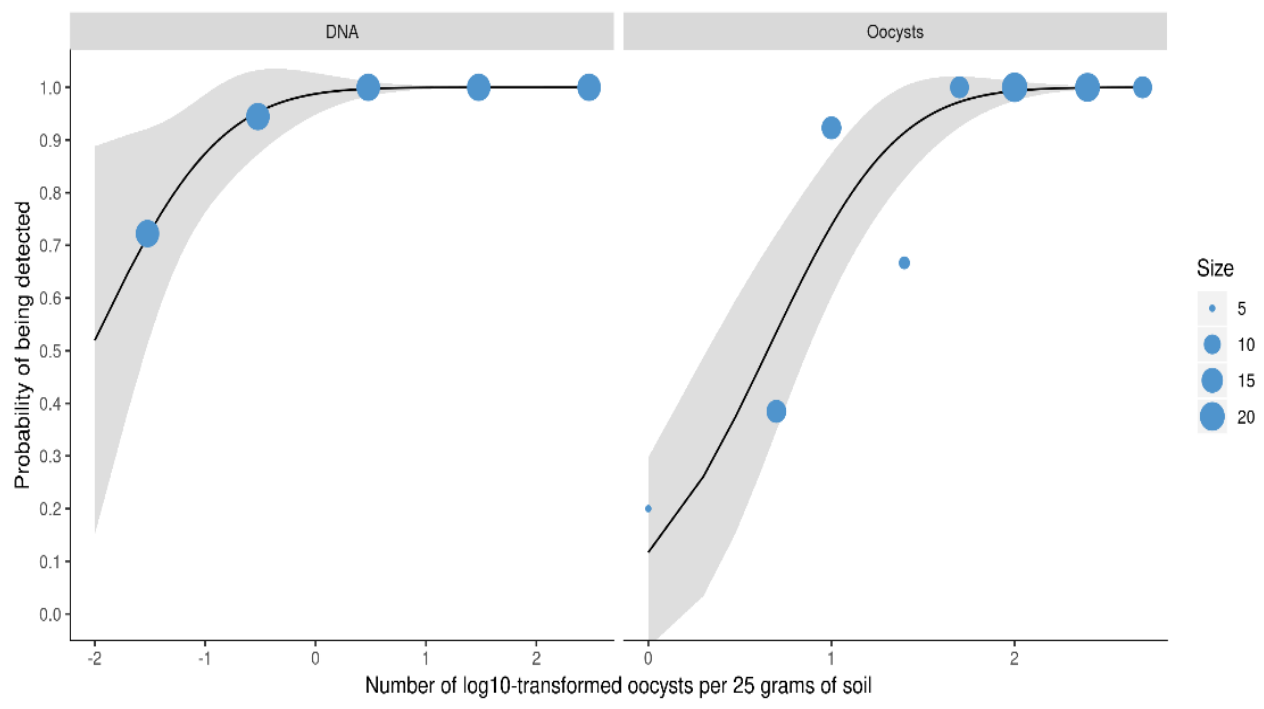

Figure 3. The log10-transformed amount of DNA added to the PCR mix and the $\log 10$-transformed number of oocysts against the probability of successfully detecting $T$. gondii DNA using the modified MC-qPCR method estimated from the probit model (size: number of replicates per group).

\subsection{Quantification of oocysts}

In the linear regression, the slope for $C q$ and $\log _{10}$ (Oocysts) turned to be significantly ( $p<0.001$ ) different (less steep) than the slope for $C q$ and $\log _{10}(D N A)$. This was likely due to the limitation of the $\mathrm{qPCR}$, with Cq values censored at around 40 . The slope is more similar considering only the soil samples spiked with high oocyst numbers, with Cq values in the same range as the DNA dilution series. Therefore, we assume that the relationships between $C q$ and $\log _{10}$ (Oocysts), $C q$ and $\log _{10}(D N A)$ share the same slope, but different intercepts (Fig. 4), as had been described previously (Opsteegh et al., 2010). The best fitting model was described below (Equation 6). The rewritten equation (7) was used to calculate the amount of oocysts present in the soil samples.

$$
\begin{aligned}
& C q=31+(12 \times \text { type })-\left(3.5 \times \log _{10}(\text { Oocysts or DNA })\right) \\
& \log _{10}(\text { Oocysts })=\frac{43-C q}{3.5}
\end{aligned}
$$




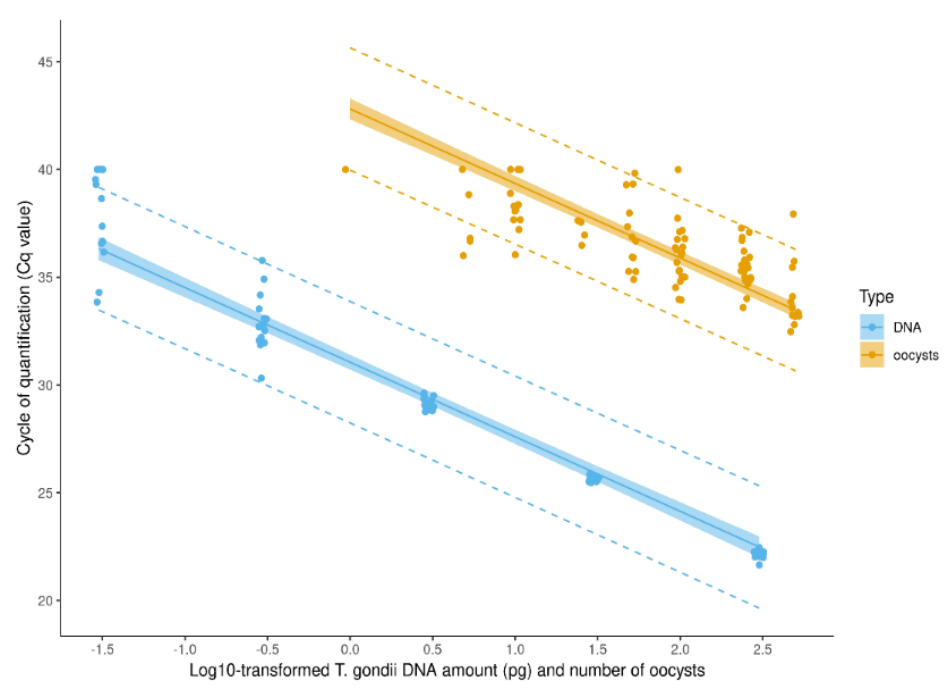

Figure 4. The log10-transformed amount of DNA added to the PCR mix and the log10-transformed number of oocysts spiked in $25 \mathrm{~g}$ soil samples against the $\mathrm{Cq}$ value measured with the qPCR. The solid line shows the mean estimated results from the fitted linear model, the confidence interval (ribbon) reflects the uncertainty around the mean estimations, and the prediction interval (dashed line) gives expected variation around a single value.

\subsection{Soil survey}

During the study period, 166 soil samples and 132 questionnaires were collected. T. gondii DNA was detected in 5 of $148(3 \%)$ interpretable soil samples $(95 \% \mathrm{Cl}$ : 1.5-7.7\%) with varying $\mathrm{Cq}$ values (Table 1). Inhibited samples (50) were additionally tested by qPCR using a 1:10 dilution of the DNA, in the end, results of 18 samples (11\%) were scored as "not determined" due to PCR inhibition. The numbers of oocysts in the five positive samples were estimated at 8, 13, 23, 48 and 478 by the linear regression.

Table 1. Description of $T$. gondii DNA positive soil samples.

\begin{tabular}{lllll}
\hline ID & Location & Sampling date & Cq value & No. of estimated oocysts \\
\hline 1 & Utrecht & $23-10-2018$ & 38.09 & 23 \\
2 & Emmen & $15-4-2018$ & 38.99 & 13 \\
3 & Utrecht & $1-3-2019$ & 36.99 & 48 \\
4 & Emmen & $1-6-2019$ & 39.60 & 8 \\
5 & Kaag en Braassem & $13-6-2019$ & 33.54 & 478 \\
\hline
\end{tabular}


Among the 132 samples with questionnaire data, the majority of them was collected in urban areas (48.5\%), followed by rural areas (17.4\%), and suburban areas (34.1\%). The five $T$. gondii DNA positive soil samples were all from backyard gardens (Fig. 5). Due to the limited number of positive samples, no further statistical analysis could be conducted on information obtained from the questionnaires.

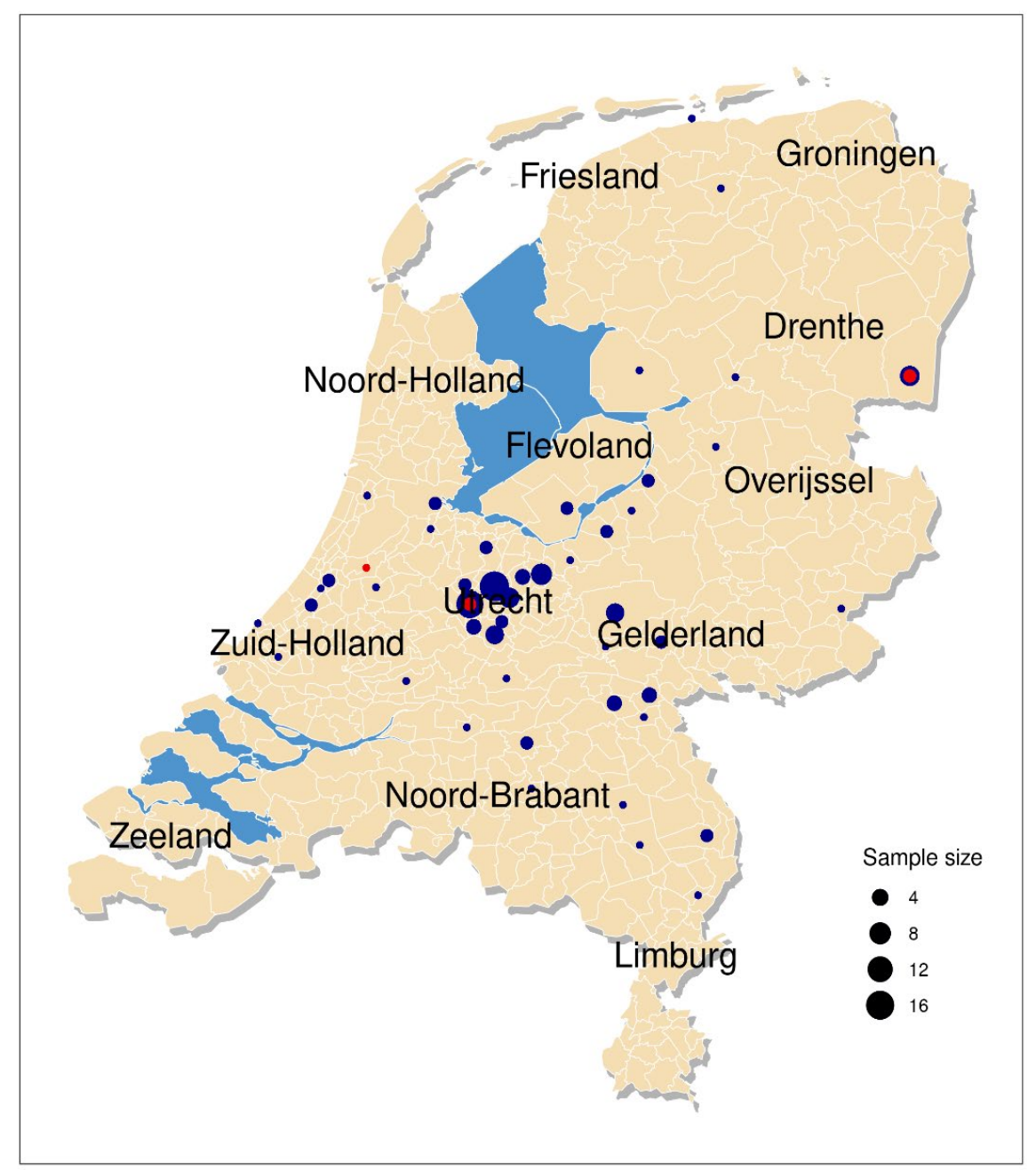

Figure 5. Locations of soil samples collected between August 2018 and November 2019 at different residential gardens or playgrounds in the Netherlands. Red dots were the locations of $T$. gondii DNA positive samples and blue dots were the locations of negative or PCR inhibited samples. 


\subsection{Risk of $T$. gondii infection via accidental ingestion of soil}

For age group one ( $\leq 10$ years), the estimated mean and SD of the corrected soil intake from individuals being exposed to soil (58\%) were 67.3 and $66.5 \mathrm{mg}$ from the approximate Bayesian computation. In our base model, with the fraction of viable $T$. gondii oocyst in soil set to 0.5 , the percentage of individuals exposed to viable oocysts was estimated at $2.0 \%$. The estimated $T$. gondii incidence rate via soil from backyard garden was 1.2 per 1,000 persons in one day. The estimated number of new infections per day in age group one ( $\leq 10$ years) was 2,182 . In the two scenarios, with the fraction of viable $T$. gondii oocyst in soil set to 0.2 and 1 , the estimated $T$. gondii incidence rates were 0.3 , and 1.8 per 1,000 persons $(\leq 10$ years) per day and number of new infections were 546 and 3,273 per day.

For age group two (>10 years), the percentage of individuals exposed to viable oocysts was estimated at $2.0 \%$ in the base model and the soil intake was half of the amount from group one. The estimated $T$. gondii incidence rate was 0.5 per 1,000 persons in one day, and the number of new infections was 5,260 . From the two scenarios, the estimated $T$. gondii incidence rates were 0.3 , and 0.7 per 1,000 persons (>10 years) per day. The estimated number of new infections in this group were 3,156 and 7,363 per day.

\section{Discussion}

In our study, a magnetic capture method for detection of $T$. gondii oocysts in soil samples was developed. The calculation of the detection probability was based on detection and non-detection results, rather than quantitative information. Thus, the $95 \%$ detection probability for the magnetic capture method should be interpreted as: $34 \mathrm{~T}$. gondii oocysts and more in $25 \mathrm{~g}$ of soil would be detected at least $95 \%$ of the time. For quantification, a linear regression model was fitted to data to obtain the relationship between $\mathrm{Cq}$ value and the $\log _{10}$-transformed amount of $T$. gondii oocysts (in this case, spiked oocysts in $25 \mathrm{~g}$ soil) or DNA (Fig. 3 ). It is worth noting that a difference between the actual number of oocysts spiked in soil and the detected number might occur, depending on the efficiency of the sample processing procedure and pipetting errors during qPCR step. In this survey, five out of 148 soil samples showed positive results. This was relatively low compared to PCR-based oocyst contamination rate from other countries (ranging from 16 to 50\% ) (Du, et al., 2012a, 2012b; Gao et al., 2016; Gotteland, GilotFromont, et al., 2014; Simon et al., 2017), regardless of the amount of soil per sample tested in those studies. The percentage of positive samples is low, despite a good $95 \%$ detection probability of 34 oocysts in $25 \mathrm{~g}$ in comparison to other detection methods. Lass et al. (2009) were able to detect 1,000 oocysts in $40 \mathrm{~g}$ of soil with a flotation test and a PCR assay targeting T. gondii B1 gene, de Wit et al. 
(2020) 100 - 1,000 oocysts in $5 \mathrm{~g}$ with sucrose flotation and a nested PCR assay targeting the internal transcribed spacer, and Lélu et al. (2011) 10 - 100 oocysts in $5 \mathrm{~g}$ of soil with sucrose flotation and a qPCR targeting the $529 \mathrm{bp}$ repeated element. Moreover, $100-1,000$ oocysts per $10 \mathrm{~g}$ of soil could be detected in $50 \%$ of the samples with the method from Afonso et al. (2008), and 10 oocysts per $10 \mathrm{~g}$ of soil could be detected in $50 \%$ of the samples with method from Escotte-Binet et al. (2019) (both used sucrose flotation and a qPCR targeting the 529 bp repeated element). In comparison, the $50 \%$ detection probability of our method was estimated to be 5 oocysts per $25 \mathrm{~g}$ soil. As soil samples were often contaminated with numbers below the $95 \%$ detection probability, it is likely that some of the negative soil samples also contained a low number of oocysts that have not been detected.

One of the advantages of sequence-specific magnetic capture approach is that it has the potential to overcome PCR inhibition (Opsteegh et al., 2010). However, for 18 samples PCR inhibition still occurred after 10 times dilution of the sample in qPCR. This might be due to the complex properties of the samples, and soil was known to contain PCR inhibitors, in particular humic substances (Sidstedt et al., 2015). In our study, we found that the MC-qPCR performed better with samples containing less sand than the sandy ones. We also found that the $\mathrm{pH}$ of soil and increasing freeze-thaw cycles did not influence $T$. gondii DNA detection. This is similar to the results published by Manore et al. (2019). They concluded that increasing freeze-thaw cycles did not increase $T$. gondii DNA detection in water and mussel tissue homogenate. In the future, more advanced sample treatment and DNA purification would be helpful to increase the detection probability for samples with low oocysts concentrations.

Together with the quantitative information obtained from this study and data from literature, a QMRA model was set up for calculating the risk of $T$. gondii infection via soil in the Netherlands. The results showed that the incidence rate ranged from 0.3 , and 1.8 per 1,000 individuals per day in age group one ( $\leq 10$ years), and 0.3 to 0.7 per 1,000 individuals per day in age group two (>10 years), and the total number of new infections ranged from 3,702 to 10,636 per day in our study. The estimated annual meat-borne $T$. gondii infections was 147,765 in the general population from the QMRA model for the Netherlands (Deng et al., 2020), which means an average of 405 new infections via meat per day. When comparing those results, the estimated number of new infections via soil was around 9-26 times higher than via meat. This makes soil a more important source of $T$. gondii infection than meat. However, this does not agree with expert elicitations (Havelaar et al., 2008) and epidemiological data from other European countries (Cook et al., 2000). 
Besides the inherent limitations based on assumptions in the model, several sources of uncertainties were identified in this study.

The soil intake data used in the calculation was based on one study which was published in 1990, investigating soil ingestion among children younger than 5 years old at daycare centers (van Wijnen et al., 1990). Although the scope of our study focused on residential gardens, it was the only study concerning soil intake among Dutch children with a big dataset, and we assume substantial changes in children's behavior are unlikely. Typically, soil intake estimates differ both between trace elements for a child within a study, and between studies. Calabrese et al. (1997) found that the most reliable tracers were aluminum and silicon. Besides the uncertainty from the accuracy of the methodology, studies concerning soil intake often used different study design, population, and some adjusted for the amount of trace element in food and medicine, contribution of indoor dust, while others did not. More recent studies have used dermal transfer to estimate the soil intake, measuring soil on hands and mouthing behavior (Ma et al., 2018). The estimated amount from this method was generally lower than methods using tracer elements (Ozkaynak et al., 2011). USEPA (2011) reviewed soil ingestion rates for children and adults from available literature and reported the recommendations of daily soil intake for assessing exposures/risks. The central tendency recommendations were $100 \mathrm{mg} /$ day for children, $50 \mathrm{mg} /$ day for adults, and $10 \mathrm{~g} /$ day for pica children (EPA, 2011). Soil-pica is defined as a form of intentional ingestion of a high amount of soil (i.e., 1-5 g/day), and geophagy is a form of soil ingestion defined as the intentional ingestion of earths usually associated with cultural practices. Studies indicated that general pica occurs in approximately half of all children between the ages of 1 and 3 years (Miao et al., 2015; Sayetta, 1986). Pica or geophagy were not considered in our QMRA due to the lack of quantitative data. Besides, soil intake estimates calculated from van Wijnen et al. (1990) were applied to an older age group ( $\leq 10$ years) and we needed to make an assumption for soil intake of age group two due to extremely limited studies (Calabrese et al., 1990; Davis and Mirick, 2006; Stanek et al., 1997) estimating adult soil ingestion.

Due to the short study periods in the published papers, the estimates of soil intake may not be representative for the long term exposure. Therefore, we focused on the incidence rate per day instead of per year using the available data. Stanek et al. (2001) used a Monte Carlo model to estimate the long-term (annual) average soil ingestion distribution using daily soil ingestion estimates from children. They found a median soil ingestion of $24 \mathrm{mg} /$ day (SD $=4 \mathrm{mg} / \mathrm{day}$ ), with the $95^{\text {th }}$ percentile of $91 \mathrm{mg} /$ day. Later, a meta-analysis conducted by Stanek et al. (2012) on data from four major studies using mass-balance method (Calabrese et al., 1989; Calabrese et al., 1997; Davis and Mirick, 2006; Davis et al., 1990) indicated that the 
mean soil ingestion for those who were exposed was $31 \mathrm{mg} /$ day (excluding zeros). These values were lower than the values we used in the QMRA model for individuals $\leq 10$ (67 mg/day), but similar to the other group (>10) (33 mg/day). Soil ingestion patterns vary depending on people's living conditions, age, frequency/type of outdoor activities, hand-to-mouth contact, or consumption and handling of fresh vegetables/fruits. Specific data for different population groups are necessary to obtain more specific results for different soil exposure routes.

Besides the scarce soil intake data, information on $T$. gondii oocyst concentration in soil was also limited. Therefore, oocyst concentration was based on only five estimates obtained from the soil survey. Due to the limited number of positive samples, further analysis on the questionnaire data could not be performed. Prior investigations have shown that $T$. gondii oocyst contamination in soil was associated with cat home (household and farm) ranges, defecation sites and season (de Wit et al., 2020; Gotteland, Gilot-Fromont, et al., 2014; Gotteland, McFerrin, et al., 2014; Simon et al., 2017). Testing soil samples from different settings would help to understand when, where and how many oocysts are present in soil. This would allow calculating specific estimates from the QMRA model to identify risk areas (e.g., playing ground, gardens and parks). That way QMRA outputs could be compared to epidemiological data, as putting sand in the mouth while playing in sandboxes and consumption of raw unwashed vegetables have been identified as risk factors for young children in the Netherlands (Hofhuis et al., 2011). In addition, soil characteristics can influence the performance of molecular detection methods, however, whether these characteristics have impacts on the viability of oocysts in soil is not clear and DNA amplification may due to the detection of dead/non-viable oocysts. The fraction of viable oocysts is not known, therefore we used a value of 0.5 in our base model. Data from Lélu et al. (2012) demonstrated that qPCR results and infectivity for mice decrease proportionally for oocysts recovered from soil using sucrose flotation and inoculated to mice, indicating that the same fraction of viability can be used for all Cq values. However, these results were concluded under experimental conditions, with flotation prior to DNA isolation and in one particular soil type (Lélu et al., 2012). Uncertainty about oocyst viability may have led to an overestimation of the estimated number of new infections per day via soil, and contributed to the unexpected result in comparison to the estimated number of infections via meat in the Netherlands (Deng et al., 2020). Even when the viability value is set to 0.1 , the estimated number of new infections per day via soil was still 9 times higher than meat-borne result. However, when the fraction of viable oocysts equals $4.5 \%$, estimated number of new infections per day via soil (603) and meat (405) were more or less equivalent. Under experimental conditions, one single oocyst has the ability to 
initiate infection in intermediate hosts (Bonačić Marinović et al., 2019; Dubey, 2006). In reality, the viability of $T$. gondii oocysts in soil may be influenced by many factors, as well as the age of the oocysts. Therefore, it is essential to develop in vitro viability assays, to avoid the use of mouse bioassay experiments and allow for large scale testing, for accurately charactering the risk of oocyst-borne $T$. gondii infection via environmental routes in the future (Rousseau et al., 2019).

In conclusion, we developed a MC-qPCR method that can be used for detecting $T$. gondii oocysts in soil and validated with an $95 \%$ detection probability of at least 34 oocysts in $25 \mathrm{~g}$ soil in residential gardens in the Netherlands. Moreover, a quantitative risk assessment model for estimating the risk of $T$. gondii infection via soil was set up. Currently the model likely overestimates the risk of infection via soil. We identified a lack of data on soil ingestion and viability data of oocysts from the environment. When we assume that $4.5 \%$ of the oocysts in soil are viable, the result from the QMRA for environmental-borne infection equals the result from the meat-borne QMRA infections. As more data on soil contamination and ingestion become available, the structure of the model can be applied to obtain more accurate estimates of the risk of $T$. gondii infection via soil.

\section{Acknowledgements}

This work was funded by the Ministry of Health, Welfare and Sport in the Netherlands, and the Netherlands Food and Consumer Product Safety Authority. The authors thank Alison Burrells and Frank Katzer from Moredun Research Institute, Scotland, United Kingdom for providing training on detection of $T$. gondii oocysts, and COST Action Short Term Mission for the financial support of the training. We also thank Peter Deplazes from Institute of Parasitology, University of Zurich, Switzerland for kindly providing $T$. gondii oocysts for the spiking experiments, Peter van Breemen and Ellen Brand from Centre for Sustainability, Environment and Health (DMG), RIVM for providing soil intake data and all the people who contributed soil samples to this study.

\section{Appendix A. Supplementary data}

Supplementary data associated with this article can be found, in the online version, at https://doi.org/10.1016/j.scitotenv.2020.143232. 


\section{REFERENCES}

Afonso, E., Lemoine, M., Poulle, M.L., Ravat, M.C., Romand, S., Thulliez, P., Villena, I., Aubert, D., Rabilloud, M., Riche, B., Gilot-Fromont, E., 2008. Spatial distribution of soil contamination by Toxoplasma gondii in relation to cat defecation behaviour in an urban area. Int. J. Parasitol. 38, 1017-1023.

Aubert, D., Villena, I., 2009. Detection of Toxoplasma gondii oocysts in water: proposition of a strategy and evaluation in Champagne-Ardenne Region, France. Mem Inst Oswaldo Cruz 104, 290-295.

Bastien, M., Vaniscotte, A., Combes, B., Umhang, G., Raton, V., Germain, E., Villena, I., Aubert, D., Boué, F., Poulle, M.L., 2019. Identifying drivers of fox and cat faecal deposits in kitchen gardens in order to evaluate measures for reducing contamination of fresh fruit and vegetables. Food Waterborne Parasitol 14, e00034.

Belluco, S., Patuzzi, I., Ricci, A., 2018. Bovine meat versus pork in Toxoplasma gondii transmission in Italy: a quantitative risk assessment model. Int. J. Food Microbiol. 269, 1-11.

Binder, S., Sokal, D., Maughan, D., 1986. Estimating soil ingestion: the use of tracer elements in estimating the amount of soil ingested by young children. Arch. Environ. Health 41, 341-345.

Bonačić Marinović, A.A., Opsteegh, M., Deng, H., Suijkerbuijk, A.W.M., van Gils, P.F., van der Giessen, J., 2019. Prospects of toxoplasmosis control by cat vaccination. Epidemics 30, 100380.

Bowie, W.R., King, A.S., Werker, D.H., Isaac-Renton, J.L., Bell, A., Eng, S.B., Marion, S.A., 1997. Outbreak of toxoplasmosis associated with municipal drinking water. The BC Toxoplasma Investigation Team. Lancet 350, 173-177.

Boyer, K., Hill, D., Mui, E., Wroblewski, K., Karrison, T., Dubey, J.P., Sautter, M., Noble, A.G., Withers, S., Swisher, C., Heydemann, P., Hosten, T., Babiarz, J., Lee, D., Meier, P., McLeod, R., 2011. Unrecognized ingestion of Toxoplasma gondii oocysts leads to congenital toxoplasmosis and causes epidemics in North America. Clin. Infect. Dis. 53, 1081-1089.

Calabrese, E.J., Barnes, R., Stanek, E.J., 3rd, Pastides, H., Gilbert, C.E., Veneman, P., Wang, X.R., Lasztity, A., Kostecki, P.T., 1989. How much soil do young children ingest: an epidemiologic study. Regul. Toxicol. Pharmacol. 10, 123-137.

Calabrese, E.J., Stanek, E.J., 3rd, Pekow, P., Barnes, R.M., 1997. Soil ingestion estimates for children residing on a superfund site. Ecotoxicol. Environ. Saf. 36, 258-268.

Calabrese, E.J., Stanek, E.J., Gilbert, C.E., Barnes, R.M., 1990. Preliminary adult soil ingestion estimates: results of a pilot study. Regul. Toxicol. Pharmacol. 12, 88-95.

Caradonna, T., Marangi, M., Del Chierico, F., Ferrari, N., Reddel, S., Bracaglia, G., Normanno, G., Putignani, L., Giangaspero, A., 2017. Detection and prevalence of protozoan parasites in ready-to-eat packaged salads on sale in Italy. Food Microbiol. 67, 67-75.

CBS, 2018. Bevolking; geslacht, leeftijd, generatie en migratieachtergrond, 1 januari. https://opendata.cbs.nl/statline/\#/CBS/nl/dataset/37325/table?ts=1588147893699

Condoleo, R., Rinaldi, L., Sette, S., Mezher, Z., 2017. Risk assessment of human toxoplasmosis associated with the consumption of pork meat in Italy. Risk Anal. 38(6):1202-1222.

Cook, A.J., Gilbert, R.E., Buffolano, W., Zufferey, J., Petersen, E., Jenum, P.A., Foulon, W., Semprini, A.E., Dunn, D.T., 2000. Sources of toxoplasma infection in pregnant women: European multicentre case-control study. European Research Network on Congenital Toxoplasmosis. BMJ 321, 142-147.

Coutinho, S.G., Lobo, R., Dutra, G., 1982. Isolation of Toxoplasma from the soil during an outbreak of toxoplasmosis in a rural area in Brazil. J. Parasitol. 68, 866-868.

Crotta, M., Limon, G., Blake, D.P., Guitian, J., 2017. Knowledge gaps in host-parasite interaction preclude accurate assessment of meat-borne exposure to Toxoplasma gondii. Int. J. Food Microbiol. 261, 95-101.

Davis, S., Mirick, D.K., 2006. Soil ingestion in children and adults in the same family. J. Expo. Sci. Environ. Epidemiol. $16,63-75$.

Davis, S., Waller, P., Buschbom, R., Ballou, J., White, P., 1990. Quantitative estimates of soil ingestion in normal children between the ages of 2 and 7 years: population-based estimates using aluminum, silicon, and titanium as soil tracer elements. Arch. Environ. Health 45, 112-122.

de Wit, L.A., Kilpatrick, A.M., VanWormer, E., Croll, D.A., Tershy, B.R., Kim, M., Shapiro, K., 2020. Seasonal and spatial variation in Toxoplasma gondii contamination in soil in urban public spaces in California, United States. Zoonoses Public Health 67, 70-78.

de Moura, L., Bahia-Oliveira, L. M., Wada, M. Y., Jones, J. L., Tuboi, S. H., Carmo, E. H., Ramalho, W. M., Camargo, N. J., Trevisan, R., Graça, R. M., da Silva, A. J., Moura, I., Dubey, J. P., \& Garrett, D. O., 2006. Waterborne toxoplasmosis, Brazil, from field to gene. Emerg. Infect. Dis., 12(2), 326-329.

https://doi.org/10.3201/eid1202.041115 
Deng, H., Swart, A., Bonacic Marinovic, A.A., van der Giessen, J.W.B., Opsteegh, M., 2020. The effect of salting on Toxoplasma gondii viability evaluated and implemented in a quantitative risk assessment of meatborne human infection. Int. J. Food Microbiol. 314, 108380.

dos Santos, T.R., Nunes, C.M., Luvizotto, M.C., de Moura, A.B., Lopes, W.D., da Costa, A.J., Bresciani, K.D., 2010. Detection of Toxoplasma gondii oocysts in environmental samples from public schools. Vet. Parasitol. 171, 53-57.

Du, F., Feng, H.L., Nie, H., Tu, P., Zhang, Q.L., Hu, M., Zhou, Y.Q., Zhao, J.L., 2012a. Survey on the contamination of Toxoplasma gondii oocysts in the soil of public parks of Wuhan, China. Vet. Parasitol. 184, 141-146.

Du, F., Zhang, Q., Yu, Q., Hu, M., Zhou, Y., Zhao, J., 2012b. Soil contamination of Toxoplasma gondii oocysts in pig farms in central China. Vet. Parasitol. 187, 53-56.

Dubey, J.P., 1995. Duration of immunity to shedding of Toxoplasma gondii oocysts by cats. J. Parasitol. 81, 410415.

Dubey, J.P., 2006. Comparative infectivity of oocysts and bradyzoites of Toxoplasma gondii for intermediate (mice) and definitive (cats) hosts. Vet. Parasitol. 140, 69-75.

Dubey, J.P., Miller, N.L., Frenkel, J.K., 1970. The Toxoplasma gondii oocyst from cat feces. J. Exp. Med. 132, 636662.

Dumetre, A., Darde, M.L., 2003. How to detect Toxoplasma gondii oocysts in environmental samples? FEMS Microbiol. Rev. 27, 651-661.

Dumetre, A., Darde, M.L., 2005. Immunomagnetic separation of Toxoplasma gondii oocysts using a monoclonal antibody directed against the oocyst wall. J. Microbiol. Methods 61, 209-217.

EFSA, 2018. Public health risks associated with food-borne parasites. EFSA Journal 16, e05495.

Ekman, C.C., Chiossi, M.F., Meireles, L.R., Andrade Junior, H.F., Figueiredo, W.M., Marciano, M.A., Luna, E.J., 2012. Case-control study of an outbreak of acute toxoplasmosis in an industrial plant in the state of Sao Paulo, Brazil. Rev. Inst. Med. Trop. Sao Paulo 54, 239-244.

EPA, 2011. Exposure Factors Handbook: 2011 Edition., in: National Center for Environmental Assessment, W., DC.

Escotte-Binet, S., Da Silva, A.M., Cancès, B., Aubert, D., Dubey, J., La Carbona, S., Villena, I., Poulle, M.-L., 2019. A rapid and sensitive method to detect Toxoplasma gondii oocysts in soil samples. Vet. Parasitol. 274, 108904.

Evers, E.G., Post, J., Putirulan, F.F., Wal, F.J.v.d., 2010. Detection probability of Campylobacter. Food Control 21, 247-252.

Frenkel, J.K., Dubey, J.P., 1973. Effects of freezing on the viability of toxoplasma oocysts. J. Parasitol. 59, 587-588.

Frenkel, J.K., Ruiz, A., Chinchilla, M., 1975. Soil survival of toxoplasma oocysts in Kansas and Costa Rica. Am. J. Trop. Med. Hyg. 24, 439-443.

Gao, X., Wang, H., Wang, H., Qin, H., Xiao, J., 2016. Land use and soil contamination with Toxoplasma gondii oocysts in urban areas. Sci. Total. Environ. 568, 1086-1091.

Gotteland, C., Gilot-Fromont, E., Aubert, D., Poulle, M.L., Dupuis, E., Darde, M.L., Forin-Wiart, M.A., Rabilloud, M., Riche, B., Villena, I., 2014. Spatial distribution of Toxoplasma gondii oocysts in soil in a rural area: Influence of cats and land use. Vet. Parasitol. 205, 629-637.

Gotteland, C., McFerrin, B.M., Zhao, X., Gilot-Fromont, E., Lélu, M., 2014. Agricultural landscapand spatial distribution of Toxoplasma gondii in rural environment: an agent-based model. Int. J. Health Geogr. 13, 45.

Guo, M., Lambertini, E., Buchanan, R.L., Dubey, J.P., Hill, D.E., Gamble, H.R., Jones, J.L., Pradhan, A.K., 2017. Quantifying the risk of human Toxoplasma gondii infection due to consumption of fresh pork in the United States. Food Control 73, Part B, 1210-1222.

Hald, T., Aspinall, W., Devleesschauwer, B., Cooke, R., Corrigan, T., Havelaar, A.H., Gibb, H.J., Torgerson, P.R., Kirk, M.D., Angulo, F.J., Lake, R.J., Speybroeck, N., Hoffmann, S., 2016. World Health Organization estimates of the relative contributions of food to the burden of disease due to selected foodborne hazards: a structured expert elicitation. PLoS One 11, e0145839.

Havelaar, A.H., Galindo, A.V., Kurowicka, D., Cooke, R.M., 2008. Attribution of foodborne pathogens using structured expert elicitation. Foodborne Pathog. Dis. 5, 649-659.

Hofhuis, A., van Pelt, W., van Duynhoven, Y.T., Nijhuis, C.D., Mollema, L., van der Klis, F.R., Havelaar, A.H., Kortbeek, L.M., 2011. Decreased prevalence and age-specific risk factors for Toxoplasma gondii IgG antibodies in The Netherlands between 1995/1996 and 2006/2007. Epidemiol. Infect. 139, 530-538.

Hohweyer, J., Cazeaux, C., Travaille, E., Languet, E., Dumetre, A., Aubert, D., Terryn, C., Dubey, J.P., Azas, N., Houssin, M., Loic, F., Villena, I., La Carbona, S., 2016. Simultaneous detection of the protozoan parasites Toxoplasma, Cryptosporidium and Giardia in food matrices and their persistence on basil leaves. Food Microbiol. 57, 36-44. 
Jones, J.L., Dubey, J.P., 2010. Waterborne toxoplasmosis - Recent developments. Experimental Parasitology 124 , 10-25.

Jones, J.L., Hanson, D.L., Dworkin, M.S., Alderton, D.L., Fleming, P.L., Kaplan, J.E., Ward, J., 1999. Surveillance for AIDS-defining opportunistic illnesses, 1992-1997. MMWR CDC Surveill. Summ. 48, 1-22.

Jones, J.L., Muccioli, C., Belfort, R., Jr., Holland, G.N., Roberts, J.M., Silveira, C., 2006. Recently acquired Toxoplasma gondii infection, Brazil. Emerg. Infect. Dis. 12, 582-587.

Jumaian, N.F., 2005. Seroprevalence and risk factors for Toxoplasma infection in pregnant women in Jordan. East Mediterr. Health J. 11, 45-51.

Lass, A., Pietkiewicz, H., Modzelewska, E., Dumetre, A., Szostakowska, B., Myjak, P., 2009. Detection of Toxoplasma gondii oocysts in environmental soil samples using molecular methods. Eur. J. Clin. Microbiol. Infect. Dis. 28, 599-605.

Lélu, M., Gilot-Fromont, E., Aubert, D., Richaume, A., Afonso, E., Dupuis, E., Gotteland, C., Marnef, F., Poulle, M.L., Dumetre, A., Thulliez, P., Darde, M.L., Villena, I., 2011. Development of a sensitive method for Toxoplasma gondii oocyst extraction in soil. Vet. Parasitol. 183, 59-67. https://pubmed.ncbi.nlm.nih.gov/?term=L\%C3\%A9lu+M\&cauthor id=20685358"

M., Villena, I., Darde, M.L., Aubert, D., Geers, R., Dupuis, E., Marnef, F., Poulle, M.L., Gotteland, C., Dumetre, A., Gilot-Fromont, E., 2012. Quantitative estimation of the viability of Toxoplasma gondii oocysts in soil. Appl. Environ. Microbiol. 78, 5127-5132.

Lin, C., Wang, B., Cui, X., Xu, D., Cheng, H., Wang, Q., Ma, J., Chai, T., Duan, X., Liu, X., Ma, J., Zhang, X., Liu, Y., 2017. Estimates of soil ingestion in a population of Chinese children. Environ. Health Perspect. 125, 077002.

Liu, X.C., He, Y., Han, D.G., Zhang, Z.C., Li, K., Wang, S., Xu, L.X., Yan, R.F., Li, X.R., 2017. Detection of Toxoplasma gondii in chicken and soil of chicken farms in Nanjing region, China. Infect. Dis. Poverty 6, 62.

Ma, J., Pan, L.B., Wang, Q., Lin, C.Y., Duan, X.L., Hou, H., 2018. Estimation of the daily soil/dust (SD) ingestion rate of children from Gansu Province, China via hand-to-mouth contact using tracer elements. Environ. Geochem. Health 40, 295-301.

Manore, A.J.W., Harper, S.L., Aguilar, B., Weese, J.S., Shapiro, K., 2019. Comparison of freeze-thaw cycles for nucleic acid extraction and molecular detection of Cryptosporidium parvum and Toxoplasma gondii oocysts in environmental matrices. J. Microbiol. Methods 156, 1-4.

Miao, D., Young, S.L., Golden, C.D., 2015. A meta-analysis of pica and micronutrient status. Am. J. Hum. Biol. 27, 84-93.

Miller, N.L., Frenkel, J.K., Dubey, J.P., 1972. Oral infections with Toxoplasma cysts and oocysts in felines, other mammals, and in birds. J. Parasitol. 58, 928-937.

Minuzzi, C. E., Portella, L. P., Bräunig, P., Sangioni, L. A., Ludwig, A., Ramos, L. S., Pacheco, L., Silva, C. R., Pacheco, F. C., Menegolla, I. A., Farinha, L. B., Kist, P. P., Breganó, R. M., Nino, B., Cardoso Martins, F. D., Monica, T. C., Ferreira, F. P., Britto, I., Signori, A., Medici, K. C., Flores Vogel, F. S., 2020. Isolation and molecular characterization of Toxoplasma gondii from placental tissues of pregnant women who received toxoplasmosis treatment during an outbreak in southern Brazil. PloS one, 15(1), e0228442.

Mughini-Gras, L., Kooh, P., Augustin, J.-C., David, J., Fravalo, P., Guillier, L., Jourdan-Da-Silva, N., Thébault, A., Sanaa, M., Watier, L., Anses Working Group on Source Attribution of Foodborne, 2018. Source attribution of foodborne diseases: potentialities, hurdles, and future expectations. Front. Microbiol. 9, 1983.

Opsteegh, M., Langelaar, M., Sprong, H., den Hartog, L., De Craeye, S., Bokken, G., Ajzenberg, D., Kijlstra, A., van der Giessen, J., 2010. Direct detection and genotyping of Toxoplasma gondii in meat samples using magnetic capture and PCR. Int. J. Food Microbiol. 139, 193-201.

Opsteegh, M., Prickaerts, S., Frankena, K., Evers, E.G., 2011. A quantitative microbial risk assessment for meatborne Toxoplasma gondii infection in The Netherlands. Int. J. Food Microbiol. 150, 103-114.

Ozkaynak, H., Xue, J., Zartarian, V.G., Glen, G., Smith, L., 2011. Modeled estimates of soil and dust ingestion rates for children. Risk Anal. 31, 592-608.

R Development Core Team, 2006. R: A Language and Environment for Statistical Computing. R Foundation for Statistical Computing, Vienna, Austria.

Rousseau, A., Villena, I., Dumetre, A., Escotte-Binet, S., Favennec, L., Dubey, J.P., Aubert, D., La Carbona, S., 2019. Evaluation of propidium monoazide-based qPCR to detect viable oocysts of Toxoplasma gondii. Parasitol. Res. 118, 999-1010.

Sayetta, R.B., 1986. Pica: an overview. Am. Fam. Physician 33, 181-185. 
Schets, F.M., van Wijnen, J.H., Schijven, J.F., Schoon, H., de Roda Husman, A.M., 2008. Monitoring of waterborne pathogens in surface waters in amsterdam, the Netherlands, and the potential health risk associated with exposure to cryptosporidium and giardia in these waters. Appl. Environ. Microbiol. 74, 2069-2078.

Shapiro, K., Bahia-Oliveira, L., Dixon, B., Dumètre, A., de Wit, L.A., VanWormer, E., Villena, I., 2019. Environmental transmission of Toxoplasma gondii: Oocysts in water, soil and food. Food Waterborne Parasitol. 15, e00049.

Sidstedt, M., Jansson, L., Nilsson, E., Noppa, L., Forsman, M., Radstrom, P., Hedman, J., 2015. Humic substances cause fluorescence inhibition in real-time polymerase chain reaction. Anal. Biochem. 487, 30-37.

Simon, J.A., Kurdzielewicz, S., Jeanniot, E., Dupuis, E., Marnef, F., Aubert, D., Villena, I., Poulle, M.L., 2017. Spatial distribution of soil contaminated with Toxoplasma gondii oocysts in relation to the distribution and use of domestic cat defecation sites on dairy farms. Int. J. Parasitol. 47, 357-367.

Sousa, S., Almeida, A., Delgado, L., Conceição, A., Marques, C., da Costa, J.M.C., Castro, A., 2020. rTgOWP1-f, a specific biomarker for Toxoplasma gondii oocysts. Sci. Rep. 10, 7947.

Stanek, E.J., 3rd, Calabrese, E.J., 1995. Daily estimates of soil ingestion in children. Environ. Health Perspect 103, 276-285.

Stanek, E.J., 3rd, Calabrese, E.J., Barnes, R., Pekow, P., 1997. Soil ingestion in adults--results of a second pilot study. Ecotoxico.I Environ. Saf. 36, 249-257.

Stanek, E.J., Calabrese, E.J., Xu, B., 2012. Meta-analysis of mass-balance studies of soil ingestion in children. Risk Anal. 32, 433-447.

Stanek, E.J., Calabrese, E.J., Zorn, M., 2001. Soil ingestion distributions for Monte Carlo risk assessment in children. Hum. Ecol. Risk Assess. 7, 357-368.

Sunnåker, M., Busetto, A.G., Numminen, E., Corander, J., Foll, M., Dessimoz, C., 2013. Approximate Bayesian computation. PLOS Compu. Biol. 9, e1002803.

Tenter, A.M., Heckeroth, A.R., Weiss, L.M., 2000. Toxoplasma gondii: from animals to humans. Int. J. Parasitol. 30, 1217-1258.

Torgerson, P.R., Mastroiacovo, P., 2013. The global burden of congenital toxoplasmosis: a systematic review. Bull World Health Organ 91, 501-508.

van Wijnen, J.H., Clausing, P., Brunekreef, B., 1990. Estimated soil ingestion by children. Environ. Res. 51, $147-162$.

Wells, B., Shaw, H., Innocent, G., Guido, S., Hotchkiss, E., Parigi, M., Opsteegh, M., Green, J., Gillespie, S., Innes, E.A., Katzer, F., 2015. Molecular detection of Toxoplasma gondii in water samples from Scotland and a comparison between the 529bp real-time PCR and ITS1 nested PCR. Water Res. 87, 175-181. 


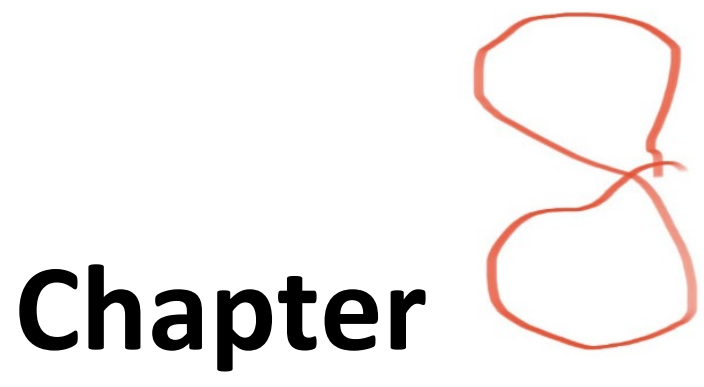

General discussion 
166 - CHAPTER 8 
T. gondii infections are prevalent worldwide and the burden of toxoplasmosis remains a public health problem that is difficult to tackle due to the complex life cycle of the parasite. The infection can be transmitted not only through a variety of food products (e.g., raw meat, vegetables, and fruits), but also through the environment (e.g., water and soil), which makes prevention and control difficult. So far, there is no commercially available vaccine for use in cats and humans, and although there is a vaccine to prevent abortions in sheep, it is not licensed to prevent tissue cysts formation in meat-producing animals. In addition, effectiveness of screening programs for pregnant women and treatment is still under debate. Therefore, primary prevention remains the most important strategy to reduce the disease burden of toxoplasmosis. The demand for effective food safety management by risk based surveillance and control programs has spread worldwide in recent decades. Quantitative evidence on important sources and transmission routes for toxoplasmosis is still lacking, but this information is needed to help identify the most cost-effective intervention and is crucial in preventing $T$. gondii infection.

This thesis focused on the relative contribution of the two main transmission routes of $T$. gondii infection via meat and soil by using a quantitative risk assessment approach. In this thesis, existing mathematical models to study transmission of the parasite and effectiveness of the potential intervention strategies were explored (chapter 2). Risk factors for $T$. gondii seropositivity in indoor-housed dairy goats in the Netherlands was estimated to evaluate whether a biosecurity intervention would be effective and feasible for reducing the risk of toxoplasmosis in grazing animals (chapter 3 ). The effect of salting on $T$. gondii viability was evaluated, and the risk of toxoplasmosis was estimated for 83 meat products for the (susceptible Dutch) general population and pregnant women (chapter 5). In addition, we implemented the conceptual QMRA model of meatborne $T$. gondii infection from the Netherlands for the mainland of China, where consumers have different consumption habits. First, data on the seroprevalence of $T$. gondii infection in pregnant women and meat-producing livestock in the mainland of China were obtained and analysed by Bayesian hierarchical modelling (chapter 4). Subsequently, those results were used as input for the QMRA model for the meat-borne toxoplasmosis (chapter 6). While focusing on tissue cysts originated infection, the transmission route via oocysts was also investigated. The risks associated with $T$. gondii exposure via soil intake for two age groups in the Netherlands were estimated. In order to obtain information about the occurrence of oocysts in soil to feed the QMRA model, a sensitive magnetic capture qPCR method was developed (chapter 7). 
In this chapter, the results obtained in the different studies are discussed in a broader perspective, including limitations of the work described in this thesis. Avenues for future research are also given.

\section{MATHEMATICAL MODELLING OF T. GONDII TRANSMISSION}

Mathematical models are useful tools to study the transmission dynamics of infectious disease in different settings, and may be used to compare the effectiveness of prevention measures. We found that the publications with mathematical models for studying the transmission dynamics and evaluating intervention strategies of $T$. gondii are scarce and only 15 studies met the inclusion criteria after full-text screening. We also found that the major limitation for several of the current mathematical models is that they do not take all relevant transmission routes in the life cycle of $T$. gondii into account. Two main intervention measures were examined in the mathematical models, i.e., cat vaccination and mouse elimination. However, the efficiencies of these intervention measures were difficult to compare, as they were evaluated in models based on different structures and parameter values. In addition, intervention measures focusing on other transmission routes (e.g., meat) were not evaluated in the models. Therefore, it is hard to draw specific conclusions about which prevention and control strategies are the most effective to reduce the burden of toxoplasmosis. The results from this study and identified data gaps can be helpful for further data collection and development of mathematical models and improve the understanding of the transmission dynamics of $T$. gondii infection.

\section{EPIDEMIOLOGICAL APPROACHES TO DEVELOP OCCURRENCE DATASETS}

Epidemiology is the study of occurrence and causes of disease at population level. Etiological epidemiological studies attempts to assess associations between exposure (risk factors) and health outcomes, and provide input for risk assessments and can also be used to validate results obtained from QMRA studies. In a cross-sectional epidemiological study in Dutch indoor-housed dairy goats, the seroprevalence in indoor-kept Dutch dairy goats was $13.3 \%$ (95\% Cl: 11.7-14.9\%), at animal level, and $61.5 \%$ (95\% Cl: $48.3-74.7 \%)$ at farm level. The number of cats and mean animal age were identified as significant risk factors related to herd seropositivity. This overall animal seroprevalence is much lower compared to the results previously reported in goats and sheep in the Netherlands and in many other countries, indicating that indoor housing seems to reduce exposure of goats to $T$. gondii. In addition, keeping cats away from the farm is expected to reduce infections in goats further. Besides, serological screening can be used to identify high-risk farms, and intervention measures (e.g., by increasing biosecurity at highrisk farms) could then be implemented in these farms to reduce the number of seropositive animals, as has been suggested for pig farms (Swanenburg et al., 
2019). Moreover, measures to inactivate tissue cysts could be applied to meat/carcasses originating from high-risk farms. Costs and benefits of these different potential interventions should be further evaluated by cost-benefit analyses, as described by Suijkerbuijk et al. (2018), or break-even analysis as described by van Asseldonk et al. (2017). However, as expected, the T. gondii seroprevalence for indoor-kept goats was not as low as has been shown for pigs (van der Giessen et al., 2007). This is likely due to less confined indoor housing (e.g., natural ventilation and introduction of bedding, silage and roughage into the stable) compared to other farm animals. These results provide important insight into $T$. gondii disease control in ruminants in the Netherlands, thereby, in the end, reducing meat-borne $T$. gondii infections in humans.

Results from the systematic review and hierarchical meta-analysis showed that $T$. gondii infection was observed in Chinese pregnant women (ranging from $2.4 \%$ to $5.0 \%$ ) and in meat-producing animals (the median seroprevalences from seven regions in pigs, chicken, small ruminant and cattle were $24,20,20$, and $9.5 \%$ respectively) in all regions of China. The use of a Bayesian hierarchical model allowed incorporating test performance (sensitivity, specificity) of the applied serological assays to estimate the adjusted prevalence by region. In addition, data from a neighboring region was used to impute seroprevalence estimates for regions that lacked information. This hierarchical framework not only provided accurate insights on $T$. gondii infection status in China, but could also be used to estimate the adjusted prevalence for other pathogens in other regions. The low seroprevalence in pregnant women implies that the majority of this age group in China are susceptible to a primary infection and their babies to congenital toxoplasmosis. These results were used as input data in the meat-borne T. gondii QMRA model for the mainland of China.

\section{MEAT AS AN IMPORTANT SOURCE OF T. GONDII INFECTION IN HUMANS}

Meat contributes several important nutrients to the diet and continues to be a significant portion of total dietary intake for many consumers. The total consumption amount per capita has been increasing globally, especially in low and middle income countries (McAfee et al., 2010; OECD-FAO, 2018). However, at the same time, meat and meat-based products are considered as one of the most important vehicles of food-borne pathogens that accounted for almost $19 \%$ of 639 outbreaks in Europe in 2017 (European Food Safety Authority, 2018). It has been reported that food-borne toxoplasmosis via consumption of undercooked or raw meat and fresh produce, may cause up to $20 \%$ of the total food-borne disease burden in the $\mathrm{EU}$ and affects more than 1 million people in the European Region each year (World Health Organization, 2015). Monitoring data from the European 
Food Safety Authority (EFSA) in 2017 showed that $T$ gondii infection is present in most livestock species and wildlife animals (e.g., pigs, sheep, goats, cattle, hunted wild boars and hunted deer) as well as the definitive host of the parasite (i.e., cats) across EU. However, knowledge about the infection status in meat-producing animals is only the start of estimating the risk of $T$. gondii infection for humans along the whole "farm-to-fork" food pathway. The risk is also dependent on other factor, such as portion size and consumption frequency, effect of meat processing and preparation habits, and the dose-response relationship. QMRA aims to bring the best available information together to estimate the probability of occurrence of a specific health effect. The World Health Organization (WHO) and the Food and Agriculture Organization have drawn guidelines on how to conduct these risk assessments of food and water (FAO/WHO, 2009). QMRA models showed that beef remains the most important source for consumers in the Netherlands, followed by pork, mutton, lamb, pork/beef mixed products, and veal, whereas, pork was the main risk to the consumers in the mainland of China, followed by meat from poultry, small ruminants, ducks and cattle. The difference between the Netherlands and China is possibly due to the population preference for different meat types and quantities of meat consumed in the two countries. However, preparation and processing is likely to differ between the Netherlands and China, but these data were not available for China and thus not included in calculations.

Ideally, the findings from our QMRA models are applied for developing risk-based intervention programs and aid risk management decision making on governmental and local level. However, both QMRA models had overestimated number of infections when compared to epidemiological data. Moreover, important knowledge gaps were identified during the development of the models. For example, crucial data was lacking about consumer exposure, consumer behavior and survival of $T$. gondii during processing (discussed below) for China. Special attention needs to be paid to those aspects when using QMRA as a routine method for measuring the annual risk estimate and source attributes.

\section{Exposure: key to better risk assessment}

Exposure assessment involves estimating the concentration of a microorganism in a medium (e.g., air, water, food), and the uptake of that medium resulting in (a dose per unit of time). One of the most difficult parts of the exposure assessment in the context of this thesis was probably estimating intake of meat. Exposure assessment in meat-borne $T$. gondii QMRA models focused on estimating the distribution of exposure to T. gondii and used data from the Dutch National Food Consumption Survey in 2007-2010 and the China Total Diet Study of 2011, respectively. However, these surveys were designed to assess nutrition status of individuals or groups, identify potential harmful chemical substances, and follow 
trends in food intake and nutrition status. Therefore, the frequency with which specific foods were consumed and prepared were not collected. Moreover, these surveys did not sufficiently cover specific risk groups for $T$. gondii infection. From the Dutch National Food Consumption Survey, the number of consumption events can be determined directly during the survey period, thus the annual number of consumption occasions can be easily extrapolated. But these data were not available for China. Thus, portion sizes and the frequency of consumption cannot be reliably estimated from the China Total Diet Study dataset. Instead of annual consumption, average daily consumption per consumer was used in the model. One of the assumptions was that meat was heated only once and there was only one serving per meat type. However, multiple small doses may have a different risk of infection in comparison to a higher dose consumed. Therefore, a scenario analysis (meat consumption amounts per livestock species were divided in two portions) was conducted. In addition, we tried to further investigate the impact of portion sizes and frequency on the estimated incidence rates and relative attribution of meat by re-structuring the model and explored two different scenarios (minimum mixing scenario and maximum mixing scenario). Results showed that portion sizes and consumption frequency did influence the incidence rate, but results obtained from the new approach led to similar conclusions about the relative attribution of different types of meat as those obtained from the basic model. For future QMRA studies, more specific questions (e.g., consumption occasions and amount) should be added to consumption surveys.

QMRA models are data hungry, and there is a real need for high quality exposure information on different phases (e.g., commercially available meat processing at retail, storage, preparation, consumption and consumers' behavior). Chardon and Swart (2016) designed a food consumption survey aimed at collecting quantitative data for QMRA applications. It includes questions about processing status at retail, consumer storage, preparation, and consumption. Unlike the traditional surveys, where only single point estimates are reported, the survey from Chardon and Swart, (2016) provided both variability and uncertainty distributions for each quantity. Furthermore, despite the availability of high quality datasets, failure to disaggregate meat from composite foods, and an assessment of meat that is purchased but not consumed (e.g., data based on sales or weights in the fridge), will substantially overestimate meat intake (Cosgrove et al., 2005; Prynne et al., 2009) and potentially underestimate risk.

\section{Predictive microbiology: can salt inactivate $T$. gondii in meat?}

Predicting growth or inactivation of foodborne pathogens under different conditions is an important part of food microbiology. In addition, predictive 
microbiological models are essential for the exposure assessment within quantitative risk assessment, especially when the concentration of the pathogens at the point of exposure cannot be easily measured directly and must be modelled. Three models for predicting the survival of $T$. gondii in meat after freezing, salting and heating were included in the Dutch meat-borne $T$. gondii QMRA. For the China QMRA model, only the effect of heating was added, since meat consumption data on specific meat products was not available from the China dataset and meat processing data is also missing.

One of the main points studied in this thesis was the new salting model. In recent years, a few studies assessed the prevalence and viability of $T$. gondii in salted meat samples and showed that for instance salt concentration and duration were associated with inactivation of $T$. gondii in meat (e.g., Genchi et al., 2017; Pott et al., 2013). However, because of the different study designs, salting treatments, and infectivity examinations of those studies, it is difficult to combine them together to quantitatively describe the effect of salting on $T$. gondii viability. There is no single model that includes the effect of all variables from different sources together. Therefore, we collected data from the literature and fitted these data in a generalized linear mixed effects model. The model estimates the effect of the most important factors (i.e., $\mathrm{NaCl}$ concentration, temperature, use of sodium nitrite, salting duration and methods) on the probability of infection in mice from salt treated meat. The work described here, as well as from the previously published meat-borne QMRA study (Opsteegh et al., 2011) clearly showed that salting had a considerable effect on $T$. gondii survival but does not necessarily inactivate all $T$. gondii. Therefore, it is important that the effects of salting in consumed or processed meat are incorporated in the quantitative risk assessment models for meat-borne toxoplasmosis, rather than excluding salted products from the risk assessment.

In spite of the fact that predictive models are very useful in exposure assessment, it should be kept in mind that all models are only a simplified representation of reality. The information needed on the inactivation parameters for $T$. gondii to develop predictive microbiology models is very specific and often determined in experimental settings. It is essential that the effects of these models are evaluated in line with industrial processing, while taking into account that different recipes and production processes may be in use to prepare the same meat product. Recently, a study has been conducted to test the effect of specific recipes on inactivation of $T$. gondii for a raw beef product which was predicted as a high risk product for human $T$. gondii infection (Opsteegh et al., 2020). Nevertheless, models could be used to guide prevention strategies, e.g., meat producers can 
adjust their recipes according to the results from models, especially those products with a high risk for human infection (e.g., filet americain, smoked sausage).

\section{T. GONDII OOCYSTS IN SOIL: A NEGLECTED SOURCE?}

Direct quantitative detection of the intake of a pathogen from the source at the time of ingestion, is not always feasible. This is particularly the case for $T$. gondii infection via oocyst ingestion by environmental contamination via soil or soil contaminated fresh produce, because the samples would rarely contain any oocysts. Even when there are a few oocysts in a sample, it can be lost due to the process for detection. Therefore, the exposure estimation to oocysts needs to rely on indirect measurement, and this begins with quantifying the occurrence of oocysts in soil. The in vivo mouse bioassay is considered the golden standard assay to demonstrate the presence of viable $T$. gondii in the target samples. It is reliable, sensitive and can be used for detecting $T$. gondii from different matrices, but the main drawback is the ethical concerns associated with distress of the animals. Moreover, the mouse bioassay is only semi-quantitative (i.e., number of infected mice per group) and the number of parasites present in the sample cannot be quantified. In addition, animal bioassays are expensive and require approval by an ethical committee, certified animal facilities and staff, and is therefore not suitable for large scale testing. To date, due to the difficulties in recovering, detecting and quantifying the number of oocysts in environmental samples, only a limited number of (molecular) methods have been developed for detecting $T$. gondii oocysts in soil, water, fresh vegetables and berry fruits samples (e.g., Afonso et al., 2008; de Wit et al., 2019; Gotteland et al., 2014; Lass et al., 2009; Liu et al., 2017; Marques et al., 2020; Wells et al., 2015), and there are currently no agreed standardized methods.

In order to feed data in the QMRA model for estimating the risk of infection via oocysts ingestion, a magnetic capture qPCR method was adapted, which is based on an earlier developed method (Opsteegh et al., 2010) to detect $T$. gondii DNA in meat samples, but optimised for oocyst detecion in soil samples. GPCR is considered as a highly sensitive detection method, but in the context of risk assessment, it is necessary to validate the performance of microbial detection methods in order to obtain accurate results from the QMRA models. One way to estimate the sensitivity of a detection method is to calculate the probability of detecting an organism when it is known to be present in the sample. Spiking experiments showed a $95 \%$ probability of detection (95\% Cl: $88-100 \%)$ of the MCqPCR method when at least 34 oocysts are present in 25 grams of soil. This is relatively more sensitive than methods reported in literature at present. Using this method, $T$. gondii DNA was detected in 5 of 148 soil samples with interpretable 
results (3\%, 95\% Cl: $1.5-7.7 \%)$ in the Netherlands. Results for 18 samples were not interpretable due to PCR inhibition. The estimated amount of oocysts presented in qPCR positive samples were quantified by a linear model, and the amount varied from 8 to 478 in 25 grams of soil. These results suggest that soil contaminated with T. gondii oocysts may be a potential source of infection in humans. However, a drawback of using a PCR based method is that it remains unknown whether the detected DNA reflects the presence of viable and infectious oocysts or not.

Compared to risk assessments for chemical agents, a key difference for microbial agents such as $T$. gondii is that they are present in discrete quantities. The shape of the distribution (counts in positive samples) should be considered explicitly. It is especially important to accurately characterize this distribution if the risk is nonlinear (i.e., the risk from ingesting 100 pathogens is very different than 100 times the risk of ingesting 1 pathogen). In the meat-borne $T$. gondii QMRA models, a beta-general distribution was used for the concentration of bradyzoites at the start of the risk assessment chain. However, for the concentration of $T$. gondii oocysts in soil, it was randomly sampled from the estimated concentration of $T$. gondii in $25 \mathrm{~g}$ of soil with replacement, due to the low number of positive samples detected in soil. In the future, more soil samples from different locations should be tested to obtain more accurate results.

The risk of infection depends not only on the occurrence of $T$. gondii oocysts in soil, but also on exposure to infectious oocysts via ingestion of soil. The qPCR method is sensitive and rapid compared to animal bioassay, but the information of infectivity is unavailable. Therefore, uncertainty is large and has a huge impact on the final result. Assuming that all the detected oocysts by PCR are infectious may lead to the overestimation of risk. Therefore, scenario analyses were used by varying the fraction of viable oocysts in the calculations. To accurately characterize the risk of oocyst-borne $T$. gondii infection via environmental routes, it is essential to determine the viability of oocysts by bioassays or in vitro viability assays. However, this is difficult for pathogens such as $T$. gondii, which cannot easily grow in tissue cultures. Moreover, another important parameter in the QMRA model is the intake of soil. Intake data collected from the literature are based on comparing the concentration of three tracer elements (titanium, aluminum, and acid insoluble residue) in soil and feces samples. We used approximate Bayesian computation to estimate the soil intake for children younger than 10 years old (group one). The approximate Bayesian computation allowed us to estimate parameters for the assumed log-normal distribution of overall soil intake for this group. However, an assumption had to be made about the amount of daily soil intake for people older than 10 due to extremely limited information for this age group. Although we obtained information about overall soil intake, the intake from different exposure 
routes, especially via fresh produce and water, remains unknown. We recommend to collect specific data for different population groups (e.g., children and people with frequent soil contact) and different pathways of exposure to oocysts in the future to obtain more specific results for different soil exposure routes.

\section{QMRA, ITS VALUE IN TOXOPLASMOSIS RISK MANAGEMENT}

As highlighted in the EFSA opinions on modernization of meat inspection, T. gondii poses an important risk to human health, and is classified as a high priority for meat inspection in pigs, sheep, goats, farmed wild boars and farmed deer (EFSA, 2013; EFSA BIOHAZ Panel, 2013). However, no EU regulation exists for the surveillance and control of $T$. gondii in meat producing animals. Results obtained from the QMRA models clearly show that data on the presence of $T$. gondii in potential sources (i.e., meat, soil) alone do not necessarily present an indication for the risk of infection. For example, the meat-borne QMRA models indicated that cooking temperature was the most influential factor related to the estimated number of $T$. gondii infections, whereas the parameter with most uncertainty; viability of oocysts from the environment, had the greatest impact on the final result in the oocyst-borne soil QMRA model. Estimating the relative importance of different sources responsible for toxoplasmosis is key for identifying appropriate interventions and for allocating resources for improving food safety and to reduce the disease burden. QMRA is a source attribution method, which aggregates all available data on different aspects together to inform risk managers about the risk of $T$. gondii infection.

Results from this thesis indicate that the importance of meat type differs depending on the country (e.g., different contamination level in livestock, consumption and preparation habits). When comparing the results calculated from meat-borne and soil-borne QMRA models in the same county, the estimated number of new infections via soil was around 9-26 times higher than via meat. This finding is different from the other approaches used for studying the source attribution of toxoplasmosis by major pathways, namely epidemiological studies (Cook et al., 2000), and expert knowledge elicitation (Hald et al., 2016; Havelaar et al., 2008; Hoffmann et al., 2017). Cook et al. (2000) showed that meat contributed $30-63 \%$ of $T$. gondii infection, while $6-17 \%$ of infections could be attributed to soil contact in pregnant women in six large European cities. Experts indicated that meat was the most important source of infection for $T$. gondii globally, but the contribution of specific meat type was estimated to vary markedly across subregions. In addition, an increasing tendency on the relative importance of soil was observed in less developed subregions (Hald et al., 2016; Hoffmann et al., 2017). In the Netherlands, experts estimated that $56 \%$ of $T$. gondii infections are 
foodborne and $36 \%$ via environmental contamination (Havelaar et al., 2008). However, a study using a serological assay to specifically detect oocyst-acquired infections has shown a high rate of oocyst acquired infections in the United States (78\%) and Chile (45\%) (Boyer et al., 2011). The relative attribution of the major transmission routes to human $T$. gondii infection is not static, but can differ by country or region (e.g., T. gondii contamination level in animals, humans and environment), age of the exposed population (e.g., age-related behavioral change), and time (e.g., change of consumer consumption preference). With increased interest in source attribution of foodborne pathogens, it is crucial to systematically summarize or combine the estimates from current methods in a comparative way for taking the correct actions.

The results obtained from source attribution approaches, eventually, can be used to support a risk management decision for setting control priorities. However, it is often not the only influential factor for the issue, many other factors can also affect the ultimate decision (e.g., cultural, ethical, and economical considerations). The most straightforward method to proceed towards decision making is perhaps via social cost-benefit analysis. It is commonly used in a variety of decision making contexts. An example of the use of $T$. gondii QMRA results in the cost-benefit analysis was presented by Suijkerbuijk et al. (2018). Here, human health, animal health and costs were expressed in monetary terms, and the costs and benefits of the two potential interventions (i.e., freezing meat intended for raw or undercooked consumption and improving biosecurity in pig farms) were evaluated by taking a range of social domains and stakeholders (e.g., consumers, producers, and government) into account and compared them with the current practice of education, especially during pregnancy. The QMRA model identified four products (i.e., filet americain, lamb chops, leg of mutton, and beef steaks) that contributed $85 \%$ of the total number of meat-borne $T$. gondii infections. These four products were included in the social cost-benefit analysis, and the net benefits of freezing for the Dutch society was calculated. The results indicated that freezing high-risk meat products (i.e., filet americain and leg of mutton) yield net benefits, while the intervention to improve the biosecurity on pig farms would result net costs (Suijkerbuijk et al., 2019). However, measures to reduce the presence of $T$. gondii in the meat products are still not in place. Freezing meat was not favoured by the Dutch consumers based on a discrete choice experiment (Lambooij et al., 2019).

Based on the knowledge and research needs identified so far, an international project called "TOXOSOURCES" was initiated in 2020. This interdisciplinary approach involves microbiologists, epidemiologists, statisticians, mathematicians and many others to estimate the relative contributions of the different sources of T. gondii infection in several European countries. As part of this project, the QMRA 
models developed in this thesis will be used to estimate and compare the relative attribution of the different sources of $T$. gondii infection in nine European countries with different consumption habits. A harmonized survey with questions developed specifically for QMRA purposes will be used for data collection. Moreover, the key knowledge gap about the role of ready-to-eat (RTE) fresh produce will be specifically addressed by developing a method and testing samples. A novel serological method that aims to specifically detect infections caused by oocysts will be developed to obtain experimental data to compare the overall importance of the environmental versus the meat-borne route. Lastly, a novel typing method to improve preparedness to detect introduction of atypical $T$. gondii strains by import and to trace the infection sources in outbreaks will be developed.

\section{CONCLUSIONS AND FUTURE PERSPECTIVES}

It was more than a hundred years ago since $T$. gondii has been discovered by scientists. The complete life cycle, and therefore also all potential sources of infection, became clear when it was found that cats (felids) are its definitive host and oocysts are excreted in feces of infected cats in the late sixties, early seventies. The relative importance of different transmission routes from different sources remained unclear, and it was less than ten years ago that QMRA was used for source attribution for different meat products. As scientific knowledge advances and consumption habits may change over time, QMRA models need to be updated regularly and should be implemented at country level, or sometimes even regionally. This work represents our current best knowledge of the relative contribution of the two main transmission routes of $T$. gondii infection via meat and soil in the Netherlands. Several limitations and data gaps were identified and elaborated in each chapter. Moreover, possible areas of research for further improvement of the models are discussed in this chapter.

The work presented in this thesis builds on the previously developed QMRA model for meat-borne $T$. gondii infections. Major improvements are the implementation of a new salting effect model in the meat-borne $T$. gondii QMRA and development of a QMRA for the other major route of infection: via exposure to oocysts in the environment. The QMRA conducted here provided the basis for risk and costbenefit analyses and can be valuable in national risk management decision making. 


\section{REFERENCES}

Afonso, E., Lemoine, M., Poulle, M.L., Ravat, M.C., Romand, S., Thulliez, P., Villena, I., Aubert, D., Rabilloud, M., Riche, B., Gilot-Fromont, E., 2008. Spatial distribution of soil contamination by Toxoplasma gondii in relation to cat defecation behaviour in an urban area. Int J Parasitol 38, 1017-1023.

Boyer, K., Hill, D., Mui, E., Wroblewski, K., Karrison, T., Dubey, J.P., Sautter, M., Noble, A.G., Withers, S., Swisher, C., Heydemann, P., Hosten, T., Babiarz, J., Lee, D., Meier, P., McLeod, R., 2011. Unrecognized ingestion of Toxoplasma gondii oocysts leads to congenital toxoplasmosis and causes epidemics in North America. Clin Infect Dis 53, 1081-1089.

Chardon, J., Swart, A., 2016. Food Consumption and Handling Survey for Quantitative Microbiological Consumer Phase Risk Assessments. J Food Prot 79, 1221-1233.

Cook, A.J., Gilbert, R.E., Buffolano, W., Zufferey, J., Petersen, E., Jenum, P.A., Foulon, W., Semprini, A.E., Dunn, D.T., 2000. Sources of toxoplasma infection in pregnant women: European multicentre case-control study. European Research Network on Congenital Toxoplasmosis. Bmj 321, 142-147.

Cosgrove, M., Flynn, A., Kiely, M., 2005. Impact of disaggregation of composite foods on estimates of intakes of meat and meat products in Irish adults. Public Health Nutrition 8, 327-337.

de Wit, L.A., Kilpatrick, A.M., VanWormer, E., Croll, D.A., Tershy, B.R., Kim, M., Shapiro, K., 2019. Seasonal and spatial variation in Toxoplasma gondii contamination in soil in urban public spaces in California, United States. Zoonoses Public Health.

EFSA, 2013. Meat inspection: EFSA completes review of practices and recommends improvements.

EFSA BIOHAZ Panel, 2013. Scientific Opinion on the public health hazards to be covered by inspection of meat from sheep and goats. EFSA Journal 1(6):3263.

European Food Safety Authority, 2018. The European Union summary report on trends and sources of zoonoses, zoonotic agents and food-borne outbreaks in 2017, EFSA Journal

FAO/WHO, 2009. Risk characterization of microbiological hazards in food: Guidelines, Microbiological risk assessment series, Rome, p. 116.

Genchi, M., Vismarra, A., Mangia, C., Faccini, S., Vicari, N., Rigamonti, S., Prati, P., Marino, A.M., Kramer, L., Fabbi, M., 2017. Lack of viable parasites in cured 'Parma Ham' (PDO), following experimental Toxoplasma gondii infection of pigs. Food Microbiol 66, 157-164.

Gotteland, C., Gilot-Fromont, E., Aubert, D., Poulle, M.L., Dupuis, E., Darde, M.L., Forin-Wiart, M.A., Rabilloud, M., Riche, B., Villena, I., 2014. Spatial distribution of Toxoplasma gondii oocysts in soil in a rural area: Influence of cats and land use. Vet Parasitol 205, 629-637.

Hald, T., Aspinall, W., Devleesschauwer, B., Cooke, R., Corrigan, T., Havelaar, A.H., Gibb, H.J., Torgerson, P.R., Kirk, M.D., Angulo, F.J., Lake, R.J., Speybroeck, N., Hoffmann, S., 2016. World Health Organization Estimates of the Relative Contributions of Food to the Burden of Disease Due to Selected Foodborne Hazards: A Structured Expert Elicitation. PLoS One 11, e0145839.

Havelaar, A.H., Galindo, A.V., Kurowicka, D., Cooke, R.M., 2008. Attribution of foodborne pathogens using structured expert elicitation. Foodborne Pathog Dis 5, 649-659.

Hoffmann, S., Devleesschauwer, B., Aspinall, W., Cooke, R., Corrigan, T., Havelaar, A., Angulo, F., Gibb, H., Kirk, M., Lake, R., Speybroeck, N., Torgerson, P., Hald, T., 2017. Attribution of global foodborne disease to specific foods: Findings from a World Health Organization structured expert elicitation. PLoS One 12, e0183641.

Lambooij, M.S., Veldwijk, J., van Gils, P., Mangen, M.-J.J., Over, E., Suijkerbuijk, A., Polder, J., de Wit, G.A., Opsteegh, M., 2019. Consumers' preferences for freezing of meat to prevent toxoplasmosis- A stated preference approach. Meat Sci 149, 1-8.

Lass, A., Pietkiewicz, H., Modzelewska, E., Dumètre, A., Szostakowska, B., Myjak, P., 2009. Detection of Toxoplasma gondii oocysts in environmental soil samples using molecular methods. European Journal of Clinical Microbiology \& Infectious Diseases 28, 599-605.

Liu, X.C., He, Y., Han, D.G., Zhang, Z.C., Li, K., Wang, S., Xu, L.X., Yan, R.F., Li, X.R., 2017. Detection of Toxoplasma gondii in chicken and soil of chicken farms in Nanjing region, China. Infect Dis Poverty 6, 62.

Marques, C.S., Sousa, S., Castro, A., da Costa, J.M.C., 2020. Detection of Toxoplasma gondii oocysts in fresh vegetables and berry fruits. Parasit Vectors 13, 180.

McAfee, A.J., McSorley, E.M., Cuskelly, G.J., Moss, B.W., Wallace, J.M., Bonham, M.P., Fearon, A.M., 2010. Red meat consumption: an overview of the risks and benefits. Meat Sci 84, 1-13.

NOS, 2019. RIVM: filet americain invriezen, dan daalt aantal medische afwijkingen.

OECD-FAO, 2018. OECD-FAO Agricultural Outlook 2018-2027.

Opsteegh, M., Dam-Deisz, C., de Boer, P., DeCraeye, S., Faré, A., Hengeveld, P., Luiten, R., Schares, G., van Solt- 
Smits, C., Verhaegen, B., Verkleij, T., van der Giessen, J., Wisselink, H.J., 2020. Methods to assess the effect of meat processing on viability of Toxoplasma gondii: towards replacement of mouse bioassay by in vitro testing. Int J Parasitol 50, 357-369.

Opsteegh, M., Prickaerts, S., Frankena, K., Evers, E.G., 2011. A quantitative microbial risk assessment for meatborne Toxoplasma gondii infection in The Netherlands. Int J Food Microbiol 150, 103-114.

Pott, S., Koethe, M., Bangoura, B., Zoller, B., Daugschies, A., Straubinger, R.K., Fehlhaber, K., Ludewig, M., 2013. Effects of $\mathrm{pH}$, sodium chloride, and curing salt on the infectivity of Toxoplasma gondii tissue cysts. J Food Prot 76, 1056-1061.

Prynne, C.J., Wagemakers, J.J.M.F., Stephen, A.M., Wadsworth, M.E.J., 2009. Meat consumption after disaggregation of meat dishes in a cohort of British adults in 1989 and 1999 in relation to diet quality. European journal of clinical nutrition 63, 660-666.

Suijkerbuijk, A.W.M., van Gils, P.F., Bonacic Marinovic, A.A., Feenstra, T.L., Kortbeek, L.M., Mangen, M.J., Opsteegh, M., de Wit, G.A., van der Giessen, J.W.B., 2018. The design of a Social Cost-Benefit Analysis of preventive interventions for toxoplasmosis: An example of the One Health approach. Zoonoses Public Health 65, 185-194.

Suijkerbuijk, A.W.M., Over, E.A.B., Opsteegh, M., Deng, H., Gils, P.F.V., Bonacic Marinovic, A.A., Lambooij, M., Polder, J.J., Feenstra, T.L., Giessen, J., Wit, G.A., Mangen, M.J., 2019. A social cost-benefit analysis of two One Health interventions to prevent toxoplasmosis. PLoS One 14, e0216615.

van Asseldonk M, van Wagenberg CPA, Wisselink HJ. Break-even analysis of costs for controlling Toxoplasma gondii infections in slaughter pigs via a serological surveillance program in the Netherlands. Prev Vet Med. 2017;138:139-146. doi:10.1016/j.prevetmed.2017.01.016

van der Giessen, J., Fonville, M., Bouwknegt, M., Langelaar, M., Vollema, A., 2007. Seroprevalence of Trichinella spiralis and Toxoplasma gondii in pigs from different housing systems in The Netherlands. Vet Parasitol 148, 371-374.

van Rossum, C.T.M., Buurma-Rethans, E.J.M., Vennemann, F.B.C., Beukers, M., Brants, H.A.M., de Boer, E.J., Ocke, M.C., 2016. The diet of the Dutch : Results of the first two years of the Dutch National Food Consumption Survey 2012-2016. National Institute for Public Health and the Environment, Bilthoven.

Wells, B., Shaw, H., Innocent, G., Guido, S., Hotchkiss, E., Parigi, M., Opsteegh, M., Green, J., Gillespie, S., Innes, E.A., Katzer, F., 2015. Molecular detection of Toxoplasma gondii in water samples from Scotland and a comparison between the 529bp real-time PCR and ITS1 nested PCR. Water Res 87, 175-181.

World Health Organization, 2015. WHO estimates of the global burden of foodborne diseases: foodborne diseases burden epidemiology reference group 2007-2015. 
180 - CHAPTER 8 


\section{ADDENDUM}

Summary

List of publication Acknowledgement About the author 
182 - ADDENDUM 


\section{Summary}

\section{English summary}

Toxoplasma gondii (T. gondii) is a protozoan parasite with a worldwide distribution. It can infect virtually all warm-blooded animals, and is the causative agent of toxoplasmosis. Humans become infected either through ingesting oocysts excreted by felids (definitive host) or consuming tissue cysts contaminated raw or undercooked meat of other warm-blooded animals (intermediate hosts). The global disease burden of toxoplasmosis is substantial and therefore considered as a public health problem worldwide. Currently, quantitative evidence on relative contribution of different transmission routes to the total disease burden is still lacking. This information is crucial to identify the most cost-effective interventions and will help allocate resources to reduce the disease burden.

Quantitative microbial risk assessment (QMRA) is one of the source attribution methods to estimate the relative importance of different sources to $T$. gondii infection. It integrates all available data on different aspects of $T$. gondii transmission together to calculate the risk of $T$. gondii infection, e.g., contamination level in sources, effect of processing methods on $T$. gondii viability, consumption pattern, and dose-response relationship.

This thesis focuses on the assessment of relative attribution of different meat products and soil to human $T$. gondii infection in the Netherlands by using QMRA models. In addition, the QMRA model of meat-borne $T$. gondii infection from the Netherlands was adapted for the mainland of China, where consumers have different consumption habits. The present thesis comprises three parts and the framework is chronological with regards to the development of QMRA models.

In the general introduction (chapter 1 ) the life cycle of $T$. gondii, source of human infection and intervention strategies are described. In addition, the advantages and disadvantages of different source attribution methods for human $T$. gondii infection are presented.

Part I of the thesis described an overview of existing mathematical models to study the transmission of $T$. gondii between different compartments and their assessments of different control strategies. A systematic review was conducted and 15 out of 484 records which met the inclusion criteria were included in the 
review (chapter 2). The identified models vary in their structures, assumptions, measured outcomes and investigated intervention strategies. Due to the complicated life cycle of the parasite (i.e., multiple hosts and different transmission pathways) and the absence of field data, models do not always represent reality. The main limitation is the failure to include all relevant transmission routes present in the life cycle of $T$. gondii. In addition, two main intervention measures (i.e., cat vaccination and mouse elimination) were examined in the mathematical models. However, the efficiencies of these intervention measures were difficult to compare, as they were evaluated in models based on different structures and parameter values. Therefore, specific conclusions about which prevention and control strategies are the most effective to reduce the burden of toxoplasmosis is still unclear. We recommend future experimental and field work to include data collection in line with data requirements for these mathematical models. Only then the identified models can be updated and extended to aid the development of effective prevention strategies.

Part II described the seroprevalence of $T$. gondii in farm animals and pregnant women, and provided relevant input data for setting up QMRA models. In chapter 3, a cross-sectional study estimating the seroprevalence and associated risk factors in indoor-housed Dutch dairy goats to investigate whether a biosecurity intervention would be feasible for reducing the risk of toxoplasmosis in grazing animals is presented. The estimated low seroprevalence suggested that indoor housing seems reduce the exposure of goats to $T$. gondii. Since number of cats on farm was clearly associated with the number of $T$. gondii seropositive goats, limiting the number of cats on the farms could reduce infection in goats. However, due to the characteristics of indoor housing systems for ruminants, increasing biosecurity in goat farms might be not as effective as for pigs and poultry. In chapter 4, a hierarchical meta-analysis was performed to estimate the seroprevalence (adjusted for test sensitivity and specificity) in pregnant women and livestock in the mainland of China. The results obtained from this chapter not only provide us accurate insight on $T$. gondii infection status in China, but were also used as important input data in the meat-borne $T$. gondii QMRA model.

Part III presented three QMRA models. The conceptual QMRA model of meatborne $T$. gondii infection in the Netherlands as described previously by Opsteegh et al. (2011) was updated based on new data on the prevalence of $T$. gondii infection in farm animals, new consumption data, and an improved $T$. gondii salting inactivation model (chapter 5). The updated model found that filet americain, spiced and smoked pork sausage and leg of mutton were the top three products associated with the highest number of predicted infections and risk per portion. Future interventions focussing on these products could have a great impact on 
reducing $T$. gondii disease burden in the Netherlands. In addition, salting had a great effect on $T$. gondii survival but does not necessarily inactive all $T$. gondii. Therefore, it is important that the effect of salting in consumed or processed meat is incorporated in the QMRA model for meat-borne toxoplasmosis. The Dutch meat-borne $T$. gondii QMRA model was adapted and implemented for the mainland of China (chapter 6 ). The model identified pork as the main source of $T$. gondii infection to the consumers in the mainland of China, followed by meat from poultry, small ruminants, ducks and cattle. Results obtained from the QMRA models clearly show that the prevalence of $T$. gondii infection in livestock does not necessarily present an indication for the risk of infection or the importance of the different types of meat on a population level. The results per country may differ depending on the consumption and preparation habits. It would be helpful if the relative attribution of the different sources of $T$. gondii infection for different countries can be estimated and compared in a harmonized risk assessment approach (e.g. using a QMRA specific consumption surveys).

After focusing on tissue cysts originated $T$. gondii infection, transmission via oocysts was also investigated (chapter 7 ). The risks associated with $T$. gondii exposure via soil intake for two age groups in the Netherlands were estimated. In order to obtain information about the occurrence of oocysts in soil to feed the QMRA model, a sensitive magnetic capture qPCR method was developed. The estimated incidence rate of $T$. gondii infection from the QMRA model via soil varied from 0.3 to 1.8 per 1,000 individuals per day. Currently the model likely overestimates the risk of infection via soil, and several data gaps were identified in the study. We recommend to collect specific data for different population groups (e.g., children and people with frequent soil contact) in the future to obtain more specific results for different soil exposure routes. Future studies should include more specific environmental transmission pathways (e.g., ready-to-eat vegetables, water, and gardening), thus the estimated relative attribution can be used to guide intervention strategies.

Finally, in the general discussion (chapter 8), main findings from all previous chapters were gathered and the values and limitations of the quantitative risk assessment approach for toxoplasmosis source attribution were discussed.

The findings in this thesis are useful for improving recognition of the risks by healthcare professionals and the public. Moreover, it can also aid science-based decision-making on where to target interventions, thereby, in the end, reducing the disease burden of toxoplasmosis. 


\section{Nederlandse samenvatting}

Toxoplasma gondii ( $T$. gondii) is een protozoaire parasiet die wereldwijd voorkomt. T. gondii kan vrijwel alle warmbloedige dieren infecteren en is de veroorzaker van toxoplasmose. Mensen raken geïnfecteerd door het binnenkrijgen van oöcysten die uitgescheiden zijn door katachtigen (eindgastheer) of door het consumeren van weefselcysten in besmet rauw of onvoldoende gaar vlees van andere warmbloedige dieren (tussengastheer). De mondiale ziektelast van toxoplasmose is aanzienlijk en wordt daarom wereldwijd gezien als een volksgezondheidsprobleem. Op dit moment is er nog onvoldoende kwantitatieve informatie over de relatieve bijdrage van verschillende transmissieroutes. Deze informatie is cruciaal om de meest kosteneffectieve interventies te identificeren en prioriteren.

Kwantitatieve microbiologische risicoschatting (QMRA) is één van de bronattributie methoden om het relatieve belang van verschillende bronnen van T. gondii infectie in te schatten. Alle beschikbare data over verschillende aspecten van de transmissie van $T$. gondii worden geïntegreerd om zo het risico van $T$. gondii infectie te berekenen. Er zijn bijvoorbeeld gegevens nodig over het besmettingsniveau in de bronnen, het effect van verwerkingsmethoden op de levensvatbaarheid van $T$. gondii, gegevens over consumptiehoeveelheden en bereidingswijzen, en de dosis-responsrelatie.

De focus van dit proefschrift ligt op het schatten van de relatieve bijdrage van verschillende vleesproducten en grond aan $T$. gondii infecties in Nederland door middel van QMRA modellen. Daarnaast is het QMRA model voor door vlees overgedragen $T$. gondii infectie in Nederland aangepast om schattingen te maken voor het vasteland van China, waar consumenten andere consumptiegewoontes hebben. Dit proefschrift bestaat uit drie delen en de structuur volgt de ontwikkeling van de QMRA modellen chronologisch.

In de algemene inleiding (hoofdstuk 1) worden de levenscyclus van $T$. gondii, de bronnen van besmetting voor de mens en mogelijke interventiestrategieën beschreven. Daarnaast worden de voor- en nadelen van verschillende bronattributie methoden voor T. gondii infectie gepresenteerd.

Deel I van het proefschrift geeft een overzicht van bestaande wiskundige modellen voor het bestuderen van de transmissie van $T$. gondii tussen verschillende compartimenten en het gebruik ervan voor de beoordeling van verschillende controlestrategieën. Een systematisch literatuuronderzoek werd uitgevoerd en 15 van de 484 publicaties voldeden aan de inclusiecriteria en werden meegenomen in het overzicht (hoofdstuk 2). De modellen variëren in hun structuur, aannames, 
gemeten uitkomsten en onderzochte interventiestrategieën. Door de gecompliceerde levenscyclus van de parasiet (meerdere gastheren en verschillende transmissieroutes) en het ontbreken van velddata, zijn de modellen niet altijd representatief voor de werkelijkheid. De belangrijkste tekortkoming is het niet volledig meenemen van alle relevante transmissieroutes in de levenscyclus van $T$. gondii. Daarnaast werden twee belangrijke interventiemaatregelen (het vaccineren van katten en het bestrijden van muizen) onderzocht in de wiskundige modellen. De efficiëntie van deze interventiemaatregelen was echter moeilijk te vergelijken, omdat deze geëvalueerd werden in modellen die gebaseerd zijn op verschillende structuren en parameterwaarden. Daardoor is het nog niet mogelijk specifieke conclusies te trekken wat betreft de meest effectieve preventie- en bestrijdingsstrategieën zijn om de ziektelast van toxoplasmose te reduceren. Voor toekomstig experimenteelen veldwerk raden wij aan om data te verzamelen overeenkomend met de datavereisten voor deze wiskundige modellen. Alleen dan kunnen de geïdentificeerde modellen bijgewerkt en uitgebreid worden en zo de ontwikkeling van effectieve preventiestrategieën steunen.

In deel II worden de seroprevalentie van $T$. gondii in vee en zwangere vrouwen beschreven. Dit zijn relevante invoerdata voor het opzetten van QMRA modellen. In hoofdstuk 3 wordt een onderzoek gepresenteerd waarin een schatting wordt gemaakt van de seroprevalentie en daaraan verbonden risicofactoren bij binnen gehuisveste Nederlandse melkgeiten. De vraag was of het mogelijk is met huisvesting onder strikte hygiënemaatregelen het risico van toxoplasmose in grazende dieren te verminderen. De geschatte lage seroprevalentie wees er op dat het binnen houden van geiten hun blootstelling aan $T$. gondii verminderd. Omdat er een duidelijk verband was tussen het aantal katten op een boerderij en het aantal $T$. gondii seropositieve geiten, zou het beperken van het aantal katten op boerderijen het aantal infecties van geiten kunnen reduceren. Echter, door de kenmerken van systemen voor het binnen huisvesten van herkauwers, kunnen hygiënemaatregelen op geitenboerderijen wellicht niet even ver worden doorgevoerd als voor varkens en pluimvee. In hoofdstuk 4 wordt een hiërarchische meta-analyse beschreven, waarmee de seroprevalentie (aangepast naar de sensitiviteit en specifiteit van de test) onder zwangere vrouwen en vee in het vasteland van China wordt geschat. De resultaten verkregen uit deze analyse geven ons niet alleen accuraat inzicht in het voorkomen van $T$. gondii in China, maar worden ook gebruikt als belangrijke invoerdata in het QMRA model voor door vlees overgedragen $T$. gondii. 
In deel III worden drie QMRA modellen gepresenteerd. Het conceptuele QMRA model van door vlees overgedragen $T$. gondii infecties in Nederland, zoals eerder beschreven door Opsteegh et al. (2011), werd bijgewerkt op basis van nieuwe data over de prevalentie van $T$. gondii infecties in vee, nieuwe consumptiedata en een verbeterd $T$. gondii zoutinactivatie model (hoofdstuk 5). Het bijgewerkte model toonde aan dat filet americain, theeworst en schapenbout de drie producten waren met het hoogste aantal voorspelde infecties en risico per portie. Toekomstige interventies die zich richten op deze producten zouden een grote impact kunnen hebben op het verminderen van de $T$. gondii ziektelast in Nederland. Daarnaast had zouten een groot effect op de levensvatbaarheid van $T$. gondii, maar inactiveert niet per se alle $T$. gondii. Het is daarom belangrijk dat het effect van zouten op vlees wordt opgenomen in het QMRA model voor door vlees overgedragen $T$. gondii. Het Nederlandse QMRA model voor $T$. gondii infecties via vlees werd aangepast en geïmplementeerd voor het vasteland van China (hoofdstuk 6). Het model identificeerde varkensvlees als de belangrijkste bron van T. gondii besmetting in het vasteland van China, gevolgd door vlees van pluimvee, kleine herkauwers, eenden en rundvlees. De resultaten verkregen uit de QMRA modellen laten duidelijk zien dat de prevalentie van $T$. gondii infecties onder vee niet noodzakelijkerwijs een indicatie geven van het risico van infectie of het belang van de verschillende typen vlees op populatieniveau. Het resultaat per land kan verschillen afhankelijk van de consumptie- en bereidingsgewoontes. Het zou nuttig zijn als de relatieve attributie van de verschillende bronnen van $T$. gondii infectie voor verschillende landen ingeschat en vergeleken worden in een geharmoniseerde risicobeoordeling (bijvoorbeeld door het gebruik van QMRA specifieke consumptie onderzoeken).

$\mathrm{Na}$ het onderzoeken van $T$. gondii infecties via weefselcysten in vlees, werd transmissie via oöcysten ook onderzocht (hoofdstuk 7). De risico's geassocieerd met blootstelling aan $T$. gondii via inname van grond werd ingeschat voor twee leeftijdsgroepen in Nederland. Om de benodigde informatie over het voorkomen van oöcysten in de grond te verkrijgen werd een gevoelige detectie methode op basis van sequentie-specifieke DNA isolatie gevolgd door qPCR ontwikkeld. Het geschatte incidentiecijfer van $T$. gondii infecties via grond van het QMRA model varieerde tussen de 0.3 en 1.8 per 1,000 individuen per dag. Op dit moment overschat het model waarschijnlijk het risico van besmetting via grond, daarnaast zijn er enkele ontbrekende gegevens geïdentificeerd in het onderzoek. Wij raden aan om in de toekomst specifieke blootstellingsdata te verzamelen voor verschillende bevolkingsgroepen om zo specifiekere resultaten te verkrijgen over de verschillende routes van blootstelling aan grond. Toekomstige onderzoeken zouden meer verschillende specifieke routes voor blootstelling aan grond moeten omvatten (bijvoorbeeld kant-en-klare groente, water en tuinieren), zodat de 
geschatte relatieve attributies ook gebruikt kunnen worden om interventiestrategieën te sturen.

Tot slot zijn in de algemene discussie (hoofdstuk 8) alle belangrijkste bevindingen van de voorgaande hoofdstukken bijeengebracht en worden de waarde en de beperkingen van bronattributie van toxoplasmose door middel van kwantitatieve risicoschatting bediscussieerd.

De bevindingen van dit proefschrift zijn nuttig voor het verbeteren van de kennis over de risico's bij medische professionals en de algemene bevolking. Bovendien kan het ondersteuning bieden bij het maken van wetenschappelijk gefundeerde besluiten over interventiemaatregelen om, uiteindelijk, de ziektelast van toxoplasmose te reduceren. 
190 · ADDENDUM 


\section{List of publications}

Deng, H., Swart, A., Bonačić Marinović, A.A., van der Giessen, J.W.B., Opsteegh, M., 2020. The effect of salting on Toxoplasma gondii viability evaluated and implemented in a quantitative risk assessment of meat-borne human infection. Int J Food Microbiol 314, 108380.

Deng, H., Exel C.E., Swart, A., Bonačić Marinović, A.A., Dam-Deisz C., van der Giessen, J.W.B., Opsteegh, M., 2020. Digging into Toxoplasma gondii infections via soil: a quantitative microbial risk assessment approach (Sci Total Environ).

Deng, H., Swart, A., Wu, Y., Li, X., Li, J., Liu, M., Opsteegh, M., van der Giessen, J. W.B., 2019. Quantitative risk assessment of meat-borne Toxoplasma gondii infection in the mainland of China. Microbial Risk Analysis 14, 100090

Deng, H., Devleesschauwer, B., Liu, M., Li, J., Wu, Y., van der Giessen, J.W.B., Opsteegh, M., 2018. Seroprevalence of Toxoplasma gondii in pregnant women and livestock in the mainland of China: a systematic review and hierarchical metaanalysis. Scientific Reports 8, 6218.

Deng, H., Dam-Deisz, C., Luttikholt, S., Maas, M., Nielen, M., Swart, A., Vellema, P., van der Giessen, J.W.B., Opsteegh, M., 2016. Risk factors related to Toxoplasma gondii seroprevalence in indoor-housed Dutch dairy goats. Prev Vet Med 124, 4551.

Bonačić Marinović, A.A., Opsteegh, M., Deng, H., Suijkerbuijk, A.W.M., van Gils, P.F., van der Giessen, J., 2019. Prospects of toxoplasmosis control by cat vaccination. Epidemics 30, 100380.

Suijkerbuijk, A.W.M., Over, E.A.B., Opsteegh, M., Deng, H., Gils, P.F.V., Bonačić Marinović, A.A., Lambooij, M., Polder, J.J., Feenstra, T.L., van der Giessen, J., Wit, G.A., Mangen, M.J., 2019. A social cost-benefit analysis of two One Health interventions to prevent toxoplasmosis. PLoS One 14, e0216615.

Meester, M., Swart, A., Deng, H., van Roon, A., Trevisan, C., Dorny, P., Gabriel, S., Vieira-Pinto, M., Johansen, M.V., van der Giessen, J., 2019. A quantitative risk assessment for human Taenia solium exposure from home slaughtered pigs in European countries. Parasit Vectors 12, 82. 
Deng, H., Wei, Y., Du, Y., Huang, Y., Zou, Z., Qin, G., He, B. Relationship between somatic cell count and milk components in buffalo milk. China animal husbandry \& Veterinary Medicine, 2013, 40 (6) 129-132. (in Chinese)

\section{SUBMITTED}

Deng, H., Cummins, R., Schares, G., Trevisan, C., Enemark, H., Waap, H., Srbljanovic, J., Djurkovic-Djakovic, O., Montiero Pires, S., van der Giessen, J.W.B., Opsteegh, M. Mathematical modelling of Toxoplasma gondii transmission: A systematic review (Food and Waterborne Parasitology)

Franssen, F., Deng, H., Swart, A., Bonačić Marinović, A.A., Liu, X., Liu, M., van der Giessen, J.W.B. Inactivation of Trichinella muscle larvae at different timetemperature heating profiles. 


\section{Acknowledgements}

Every time I get a copy of a PhD thesis, the first two things I do are to check if there are nice figures, and then go to the acknowledgement to find familiar names. At the time, I already decided that I want to have colored figures if I ever write a thesis and believed that I probably would have a short list of names in the acknowledgement. After all, most of the hard work will be done by the author, I thought.

Then, I started my own PhD journey!

This work is a synergistic product of many minds, and the journey to produce it would have been no fun without the support from my treasured colleagues, friends and family.

\section{SUPERVISION}

It all began when I started my internship project for my master study at UU. Through my supervisor at that time, Mirjam Nielen, I got the chance to study one of the most successful parasites in the world at RIVM, and was supervised by two amazing researchers, who later became the supervisors of my PhD study as well.

Without the guidance from Joke van der Giessen, the whole thesis wouldn't have existed. She has a clear mind and sees the big pictures. She laughs about me not knowing how to make schedules, and knew when to pull me back on track from daydreaming. On her sabbatical leave, we spent two nice weeks together in China, talking, eating local food, and getting foot massages. I am so grateful that she has taken great care of me under her wing.

Marieke Opsteegh is definitely my role model. I admire her excellent epidemiology knowledge and writing skills. Her door was always open to me and she always had an answer for my questions, even like "maybe Google it". After she checked the manuscript, I felt like it became award-winning level. My biggest regret is that I missed the deadline to nominate her as the Supervisor of the Year!

I met my promotor Dick Heederik later in Joke's office. Actually, I was in his class learning about risk assessment and management during my master study already. His broad area of expertise amazes me, and his calm manner influences me. He is always there, when I need him. 


\section{DATA ANALYSIS}

I tell people that Arno Swart is my "sensei" for modeling. I am totally addicted to data analysis because of his magic-like modeling skills. The forbidden word for Arno is (detection) limit. When it comes to solving modeling problems, it feels like he can come up with unlimited number of ideas. And me, the tireless implementer of them. The most raging weather I experienced in the Netherland was the brainstorms brought by Arno and Axel Bonačić Marinović. One time it even lasted till 6 pm! In my eyes, it was absolutely not normal in Dutch culture. What can I say? It has to be passion.

When I started my first month, Joke sent me to Florida to learn how to build QMRA models from Arie Havelaar and Brecht Devleesschauwer. That was my first time in America. Arie kindly picked me up from the airport and showed me around the campus. But later, he brought me to places full of wild alligators and bats! Undoubtedly, my first close life experience with a top expert/professor was thrilling. I am more than excited to know that he is in the assessment committee. So, better be prepared. After the course, I started my first project. Brecht generously offered help for building the Bayesian hierarchical model, with his free time! Brecht, I am indebted to you.

\section{WORKING IN THE LAB}

I started learning lab skills by just standing behind Cecile Dam-Deisz in the parasitology lab during my internship. Her neat and highly organized style will always be my example for working in the lab. Above all else, she endlessly walked me from her desk to the lab to show me where the capture oligos are in the freezer (same spot for decades). I truly admire her patience.

Through the funding I got from COST Action, I went to Moredun, Edinburgh, to learn how to detect $T$. gondii oocysts in water from Frank Katzer and Alison Burrells. Everything was fine there, even the weather. However, eating and drinking were not allowed in the office! Therefore, I cherished the coffee breaks spent talking with them more than ever.

Kitty Exel saved me from the lab, so that I can work on my beloved numbers behind the screen. She is passionate about lab work as well as little bad jokes. Last I heard she was excited about the trip to Copenhagen working on an economic study and enjoyed her own PhD a lot, no joke.

\section{PARASITOLOGY}

I went to Jilin University via the China Exchange Program, where I spent a unforgettable half-year in the lab of Mingyuan Liu. I am grateful to have met so 
many great people there, and will cherish the memories of our time together. Besides the main line of my PhD study, I was involved in the COST Action, Toxoscan project and the Dutch $T$. gondii working group. I have learned a lot from these groups of wonderful researchers. Most importantly, working with them has always been a pleasure for me.

\section{COVER DESIGN}

I love the cover of this thesis. Because my unbelievably sweet cat Ruby is on the front, and back! Surely, the designer/go teacher Kim Ouweleen (www.murugandi.com) did a brilliant job. Even after I only said: "Something like 管 中窥豹, 可见一斑”. Where does this mind reading talent come from? Probably years of practicing go is the answer.

\section{TRANSLATION}

Ten years ago, I first met Tim van Vliet in a Mexican restaurant/bar in Beijing. I immediately knew he is smart. I was totally surprised that he agreed to translate the summary in Dutch. He usually tries to avoid reading my papers, which I secretly assume is because the smart man (as a former sinologist, current Java developer) hasn't done the right education for my stuff. "Well, he is at least good at languages", I thought.

\section{REVIEWING}

The constructive (sometimes tough) suggestions and comments from all the anonymous reviewers of every manuscript certainly made the final product a better piece. I am also grateful to the review/assessment committee for their time and effort spent on evaluating this thesis.

\section{EMOTIONAL SUPPORT}

I went to my first scientific conference in England with my bosses Arjen van de Giessen and Kitty Maassen. It was a fun and exciting trip for me, because I won the prize for best poster, in front of my bosses! The consequence was that I've been "promoted" from the 2nd floor to the 3rd floor shortly after we went back.

Since the promotion, my $\mathrm{PhD}$ life changed from black/white to rainbow. Not only did I often see rainbows out of my window, but more importantly the two out of millions roommates: Frits Franssen and Manoj Fonville. Because of them, the room is full of wisely talks, eruptive laughing and worried plants.

My dear friends, Ryanne, Tryntsje, Robin, Sjors, Linda, Renjie, Martien, 郭捷, 聂 
培 and all the dear colleagues at Z\&O, I enjoyed the time we spent together very much, and it always feels like I was home.

Arno and Paul, thank you so much for daring to stand $1.5 \mathrm{~m}$ behind me as paranymph! It means a lot to me.

Het is gek dat ik vijf jaar in het huis van Sjaak en Yolanda heb gewoond! Ik heb alle Nederlandse gewoontes van hen geleerd: de kalender ophangen in de wc, verjaardagskaarten verzenden, Nederlands avondeten: aardappelen, bloemkool en gehaktballetjes, etc. Dit heeft mij enorm geholpen om de Nederlandse cultuur te begrijpen. Het is opmerkelijk dat zij me nog steeds niet beu zijn na vijf jaar voor mij de was te hebben gedaan. Ik ben ook dankbaar voor de geweldige tijd die ik met Chantal, Edwin en Gwen heb doorgebracht.

爸爸妈妈, 你们对待重要事情深思熟虑的态度和勤俭耐劳的生活作风，是我 一直学习的榜样。琳姐、子龙, 我以你们为姐弟而骄傲! 姐夫、赛赛, 很高 兴你们加入了这个大家庭，谢谢你们一如既往的支持和鼓励。沐沐、荣格、 感谢你们给姑姑 / 小姨论文每一章的封面添加了一些小乐趣。嗲㕬, 没有你 就不会有我，谢谢你让我度过了长达三十二年的快乐童年。和你有关的记 忆, 我会一直珍藏在心里。

Timmy, 是你绝不越沮代扈的行为准则, 促使我成长为一名独立的研究员。 我的勋章有你一半!

Usually, I avoid using exclamation marks, but I have loosened my standard this time. Thank you for reading it. 


\section{About the author}

Huifang Deng was born in Changsha, China, on 6 May 1988. After receiving her bachelor's and master's degree in Veterinary Medicine and Clinical Veterinary Medicine in China in 2010 and 2013, she moved to the Netherlands for a postgraduate programme of Veterinary Epidemiology and Economics at Utrecht

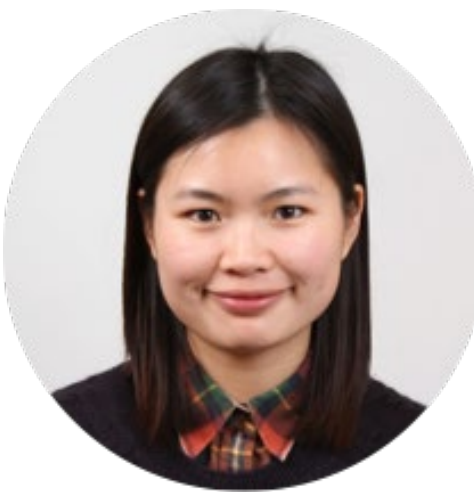
University, for which she has been awarded the degree of Master of Science in 2015. During her studies, she gained interest in public health and data analysis. From 2016 to 2017, she was granted an Exchange Programme by the Royal Netherlands Academy of Arts and Sciences, during which she worked as a junior researcher at the Dutch National Institute for Public Health and the Environment (RIVM) and Jilin University. During this time, she also received training at the University of Florida in performing quantitative microbial risk assessments. After this one-year programme, she started working at RIVM on her PhD project. The results of her $\mathrm{PhD}$ research on the source attribution of human toxoplasmosis are described in this thesis. During the PhD project, Huifang was granted a Short Term Scientific Mission by the European Cooperation in Science and Technology, and received training in the lab at Moredun Research Institute. In addition, she was involved in different projects within and outside RIVM. She was the coordinator of the Dutch Toxoplasma working group and of a systematic review performed in a European consortium. 
198 - ADDENDUM 
$\because$

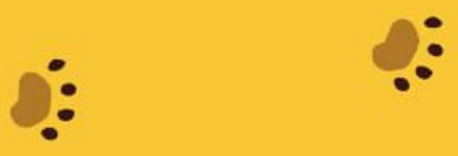

0

0

0

$: 0$

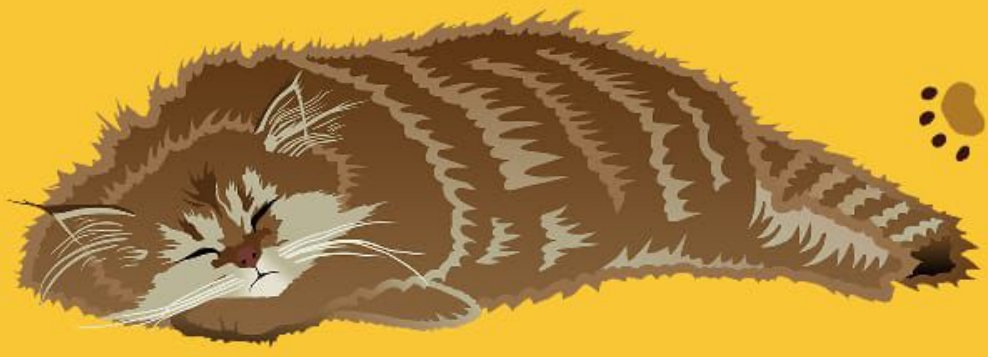

$\because$ 